\title{
Towards Stochastic and Deterministic Modeling of Mechanical Waves in Disordered Media
}

Impulse Propagation:

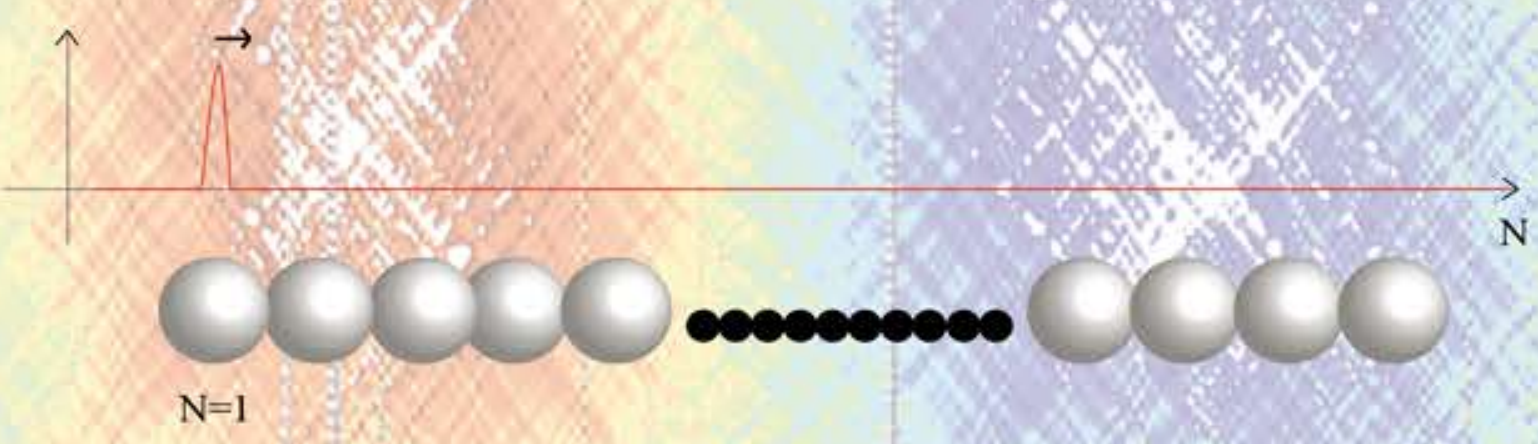

Standing Wave:

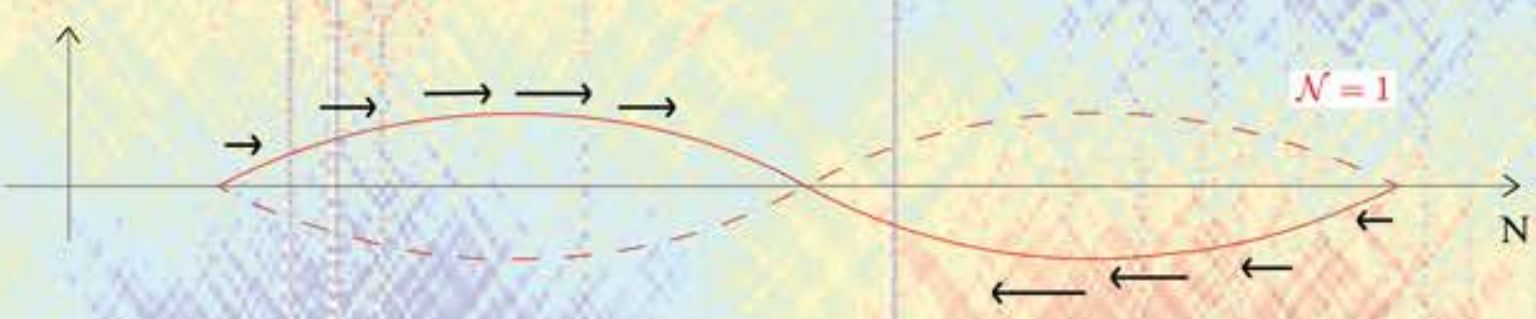




\section{Towards Stochastic and Deterministic Modeling of Mechanical Waves in Disordered Media}

Rohit Kumar Shrivastava 
Towards Stochastic and Deterministic Modeling of Mechanical Waves in Disordered Media

Rohit Kumar Shrivastava

PhD thesis, University of Twente, The Netherlands.

Copyright (C) 2018 by Rohit Kumar Shrivastava. All Rights Reserved. No parts of this thesis may be reproduced or transmitted in any form or by any means without the permission of the author or the publishers of the included scientific papers.

ISBN: 978-90-365-4617-1

DOI number: 10.3990/1.9789036546171

Cover design by Rohit Kumar Shrivastava, adapted from Fig. 3.1, 3.9(b) and $3.10(\mathrm{~b})$

Keywords: Particle Scale Model, Discrete Element Method, Mechanical Wave Propagation, Spectral Analyses, Disorder, Photoelasticity

This work is a part of the Industrial Partnership Programme (IPP) "Computational sciences for energy research" of the Foundation for Fundamental Research on Matter (FOM), which is part of the Netherlands Organisation for Scientific Research (NWO). This research programme is cofinanced by Shell Global Solutions International B.V.

Printed by Ipskamp Printing, Enschede, The Netherlands. 
TOWARDS STOCHASTIC AND DETERMINISTIC MODELING OF MECHANICAL WAVES

IN DISORDERED MEDIA

\section{PROEFSCHRIFT}

ter verkrijging van

de graad van doctor aan de Universiteit Twente, op gezag van de rector magnificus, prof. dr. T.T.M. Palstra

volgens besluit van het College voor Promoties

in het openbaar te verdedigen

woensdag 20 September 2018 om 16.45 uur

door

Rohit Kumar Shrivastava

geboren op 6 march 1989

te Rampur, India 
Dit proefschrift is goedgekeurd door de promotor:

Prof. dr. rer.-nat. S. Luding

Samenstelling promotiecommissie:

Prof. dr. Geert P.M.R. Dewulf

Prof. dr. rer.-nat. Stefan Luding

Prof. dr. ir. Ton van den Boogaard

Prof. dr. ir. André de Boer

Prof. dr.-ing. Holger Steeb

Prof. dr. Catherine O'Sullivan

Dr. Kuniyasu Saitoh

Peter Hoogerbrugge
Universiteit Twente, voorzitter/secretaris Universiteit Twente, promotor

Universiteit Twente

Universiteit Twente

Universität Stuttgart

Imperial College London

Tohoku University

Shell Int. Projects and Technology B V. 
For the ambition and penance of my mother... For the cooperation and patience of my father... For the trust and perseverance of my Professor... For the love and support of my friends... Plus Ultra... 



\section{Contents}

1 Introduction 1

1.1 Dispersion . . . . . . . . . . . . . . . . . 2

1.2 Energy Loss (Attenuation) in Mechanical Waves . . . . . . . . 3

1.3 Modes of Mechanical Waves . . . . . . . . . . . . . . 4

1.4 Signature of Mechanical Wave Propagation in Disordered Media 5

1.5 Mesoscopic Wave Phenomena: Diffusion, Coherent Backscattering Effect \& Weak Localization . . . . . . . . . . . . . . . 7

1.6 Disordered Media: Granular Media . . . . . . . . . . . . . 8

1.6.1 Modeling for Structure and Dynamic Analyses . . . . . . 9

1.6.2 Experimental Investigations . . . . . . . . . . . . . 9

1.7 Stochastic Modeling . . . . . . . . . . . . . . . . . 11

1.8 Nonlinear Phenomena . . . . . . . . . . . . . . . . . . . . 12

1.9 Thesis Outline . . . . . . . . . . . . . . . . . 13

2 Effect of Mass Disorder on Bulk Sound Wave Speed: A Multiscale Spectral Analysis $\quad 15$

2.1 Introduction . . . . . . . . . . . . . . . . . 16

2.2 Modeling a general one-dimensional chain $\ldots \ldots \ldots \ldots . . \ldots$

2.2 .1 Non-dimensionalization . . . . . . . . . . . . 20

2.2.2 Equation of Motion : Nonlinear (Hertzian) . . . . . . . 21

2.2.3 Equation of Motion : Linear . . . . . . . . . . . . . . 21

2.2.4 Analysis in real space/spatial Fourier space : . . . . . . 23

2.2.5 Initial Conditions : Impulse Driving . . . . . . . . . 24 
2.2.6 Mass Distribution, Disorder Parameter $(\xi)$, Ensemble Averaging \& Binning . . . . . . . . . . . . 25

2.2.7 Participation Ratio \& Localization Length . . . . . . . . . 26

2.2 .8 Dispersion . . . . . . . . . . . . . 27

2.2 .9 Total Energy Dispersion . . . . . . . . . . . . . . 27

2.2 .10 Group velocity . . . . . . . . . . . . . . . . . . . 28

2.3 Results \& Discussions . . . . . . . . . . . . . . . 28

2.3.1 Nonlinear (Hertz) and Linear Space Time Responses . . . 29

2.3.2 Displacement Response of the Three Distributions . . . . . 30

2.3.3 Displacement Response for Different Disorder . . . . . . . 32

2.3.4 Coherent Wave Speed and Disorder . . . . . . . . . . . 34

2.3.5 Frequency Response \& Dispersion . . . . . . . . . . . 36

2.3.6 Total Energy Dispersion in Disordered Chains . . . . . . 39

2.3.7 Participation Ratio \& Localization length . . . . . . . . . 39

2.3.8 Total Energy of Eigenmodes . . . . . . . . . . . . . . 41

2.4 Conclusions . . . . . . . . . . . . . . . . . . . 42

3 Energy Propagation with Distance and Across Wavenumbers in a Granular Chain

3.1 Introduction . . . . . . . . . . . . . . . 52

3.2 Granular Chain Model . . . . . . . . . . . . . . . . . 53

3.2.1 Linearized Equation of Motion . . . . . . . . . . . 55

3.2 .2 Impulse Propagation Condition . . . . . . . . . . . . 57

3.2 .3 Standing Wave Condition . . . . . . . . . . 57

3.2.4 Mass Disorder/Disorder Parameter $(\xi)$ \& Ensembles ... . 58

3.3 Energy Evolution . . . . . . . . . . . . . . . . . . . 59

3.3.1 Total Energy in the Wavenumber Domain . . . . . . . . . 60

3.3.2 Numerical Master Equation . . . . . . . . . . . . . . 61

3.4 Results . . . . . . . . . . . . . . . . . . . 62

3.4.1 Energy Propagation with Distance . . . . . . . . . 62

3.4.2 Energy Propagation across Wavenumbers: Master Equation for Spectral Energy . . . . . . . . . . . . . . . . 67

3.5 Conclusion . . . . . . . . . . . . . . . 75

4 Rotational Sound Propagation in Disordered Frictional Disks 77

4.1 Introduction . . . . . . . . . . . . . . 78

4.2 Numerical Method . . . . . . . . . . . . . . . . . . . . . . . 79

4.3 Numerical Results . . . . . . . . . . . . . . . . . . . . . 80

4.4 Theoretical Model . . . . . . . . . . . . . . . 85 
4.5 Discussion and Conclusion . . . . . . . . . . . . . . . . 91

5 Long Wavelength Coherent Pulse of Sound Propagating in Granular Media 93

5.1 Introduction . . . . . . . . . . . . . . . . . 94

5.2 Micromechanical Model: Granular Chain _ . . . . . . . . . 96

5.2.1 Non-Dimensionalization . . . . . . . . . . . . . . . 97

5.2 .2 Nonlinear Equation of Motion . . . . . . . . . . . . . . 97

5.2 .3 Hertzian Equation of Motion _ . . . . . . . . . . . . 98

5.2 .4 Linearized Equation of Motion . . . . . . . . . . . 98

5.2.5 Disorder in the Chains and Ensemble Averaging . . . . . 100

5.3 Numerical Results and Discussions . . . . . . . . . . . . 100

5.3.1 Ensemble Averaged Space Time Responses and the Coherent Wavefront . . . . . . . . . . . . . . . . . . 101

5.3.2 Width of the Coherent Wavefront . . . . . . . . . . . 102

5.3.3 Coherent Wavefront Velocity _... . . . . . . . . 106

5.4 Experimental Construction . . . . . . . . . . . . . . . . 108

5.5 Force Measurements . . . . . . . . . . . . . . . . . . . . . . 109

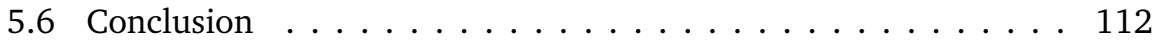

6 Conclusions and Outlook 113

A Total Energy Harmonic Evolution 117

B Hertz contact model 119

C Matching the first two moments of different distributions (normal, uniform and binary). 121

C.1 Normal Distribution . . . . . . . . . . . . . . . . . . 122

C.1.1 First Moment . . . . . . . . . . . . . . . . . . . . . . 123

C.1.2 Second Moment . . . . . . . . . . . . . . . . . . . 123

C.2 Binary Distribution . . . . . . . . . . . . . . . . . . . . 124

C.2.1 First Moment . . . . . . . . . . . . . . . . . . 125

C.2.2 Second Moment . . . . . . . . . . . . . . . . . . 125

C.3 Uniform Distribution . . . . . . . . . . . . . . . . . . . 126

C.3.1 First Moment . . . . . . . . . . . . . . . . . . . 126

C.3.2 Second Moment . . . . . . . . . . . . . . . . . 127

D Energy Equations in the Matrix Form 129 
$\begin{array}{lr}\text { Summary } & 157\end{array}$

$\begin{array}{lr}\text { Samenvatting } & 159\end{array}$

$\begin{array}{ll}\text { Acknowledgments } & 161\end{array}$

$\begin{array}{ll}\text { Curriculum Vitae } & 163\end{array}$ 


\section{Chapter 1}

\section{Introduction}

It ain't what you don't know that gets you into trouble. Its what you know for sure that just ain't so.

Mark Twain

A mechanical wave is propagation of vibration with transfer of energy and momentum. The branch of physics which deals with the study of mechanical waves is acoustics [37]. The theory of sound/mechanical wave propagation has been studied since 1877 [156] to understand the properties of the underlying propagating media. Examples are:

- Internal structure of Earth (Earthquakes which are another form of naturally occurring mechanical waves have been used to speculate the composition of Earth beneath the surface, the study is called seismology [5, 34, 174]).

- Oil/gas exploration (artificial mechanical waves are sent into the Earth using vibroseis (a vibrator mounted truck) or dynamite and the responses are received using geophones, the received responses are analyzed to find buried objects [85, 224]).

- Non-destructive testing of materials for geotechnical investigations (artificial mechanical waves are sent into the material and the received response is studied using piezoelectric devices such as electronic transducers [215] or bender elements $[142,176])$. 
- Non-invasive examination of the human body [12, 138, 209].

- Detecting evolution of cracks in concrete cement structures (Coda wave interferometry [92, 99, 183]).

- Designing metamaterials for shock protection [46, 49, 59, 71, 137], as acoustic waveguides [25], for energy focusing/trapping applications [12, $46,95]$, etc.

The propagation of vibrations in solids is accompanied by various phenomena such as dispersion, attenuation (intrinsic and scattering), geometric spreading, transfer of energy across different modes of mechanical waves (longitudinal or P-wave, shear or S-wave, rotational waves, surface waves or Rayleigh and Love waves), frequency filtering, localization, solitary wave propagation (nonlinear effect), etc. These phenomena are discussed in detail in the upcoming sections $(1.1,1.2,1.3,1.5)$. The signatures or characteristics of a sound signal (time series of displacement or velocity) in a disordered media are mentioned and illustrated in section 1.4. The propagating media are often disordered and granular in nature as discussed in section 1.6; the motivation of the choice of such disordered media, their omnipresence and the basis behind modeling such a media is elaborated; experimental investigations carried out in the past few decades and the role of photoelastic disks in structure and dynamic analyses of granular media are highlighted. In section 1.7, the need for stochastic modeling of mechanical waves will be discussed. Some nonlinear phenomena related to mechanical waves (solitary wave propagation, different sound velocity scaling, etc.) are mentioned in section 1.8 as these phenomena have caught a lot of attention in the recent decades worth mentioning. Finally, the outline of the thesis is presented in section 1.9.

\subsection{Dispersion}

Different frequencies of mechanical waves in disordered media travel with different velocities; this phenomena is known as dispersion. It occurs as a result of multiple scattering of waves from the internal heterogeneity of the disordered media [161]. Sachse et al. 1977 [161] classifies dispersion into 5 types:

1. Geometric Dispersion: It occurs due to specimen boundaries.

2. Material Dispersion: It occurs due to frequency dependence of material parameters like bulk moduli, shear moduli, mass density, etc. 
3. Scattering Dispersion: It occurs due to scattering of waves from the internal scatterers present in the media.

4. Dissipative Dispersion: It occurs due to absorption of mechanical wave energy into heat or any other form of irreversible process.

5. Nonlinear Dispersion: It occurs when the wave velocity is dependent on the wave amplitude.

Dispersion occurring as a result of discretized media can be identified as a combination of material and scattering dispersion. When we talk about dispersion, we often come across terminologies such as phase velocity or group velocity. The phase velocity is defined as $v=\frac{\omega}{k}$ where $\omega$ is the frequency and $k$ is the wavenumber. Dispersion causes broadening of pulses and the mechanical waves propagate in the form of a wave packet (series of wave trains), the velocity of this wave packet as a whole is defined as group velocity and is given by $v_{g}=\frac{\partial \omega}{\partial k}$. Phase velocity or group velocity can be obtained by taking slope or tangent of $\omega$ vs. $k$ curves.

\subsection{Energy Loss (Attenuation) in Mechanical Waves}

Energy attenuation of mechanical wave packets occur due to various mechanisms which can be broadly classified into 3 categories: geometric spreading, intrinsic attenuation and scattering attenuation [174, 218]. Geometric spreading is the decay of energy due to propagation of vibration in different directions. Scattering attenuation is the decrease in energy due to spreading of energy across different frequencies. Intrinsic attenuation is the loss of energy in the form of dissipative mechanisms such as mechanical heat generation. Isolating these mechanisms and understanding their effects independently has been the goal of wave propagation models. One dimensional modeling assists in removing geometric/directional effects while analyzing energy transfer across frequencies, which has been done in the following chapters in the thesis. Some of the models to estimate scattering attenuation have been mentioned in [84]. For intrinsic attenuation, power law models have been used to quantify the loss of energy [193].

A parameter which has been used often to quantify attenuation is the Quality Factor $(Q)$ or its inverse $\left(Q^{-1}\right)[88,159,198,199]$. If $A(\omega)$ is the Fourier amplitude of a signal, then, $|A(\omega)|^{2}$ is its power/energy; the relationship be- 


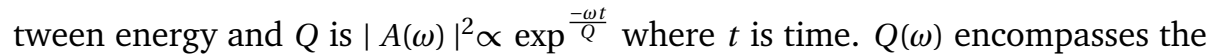
frequency dependence of both scattering as well as intrinsic attenuation.

\subsection{Modes of Mechanical Waves}

Mechanical waves have different modes through which the transfer of energy and momentum takes place. The particle motion of the underlying media is instrumental in defining these modes. The modes are classified broadly into bulk waves (referred to as body waves in case of seismology) and surface waves.
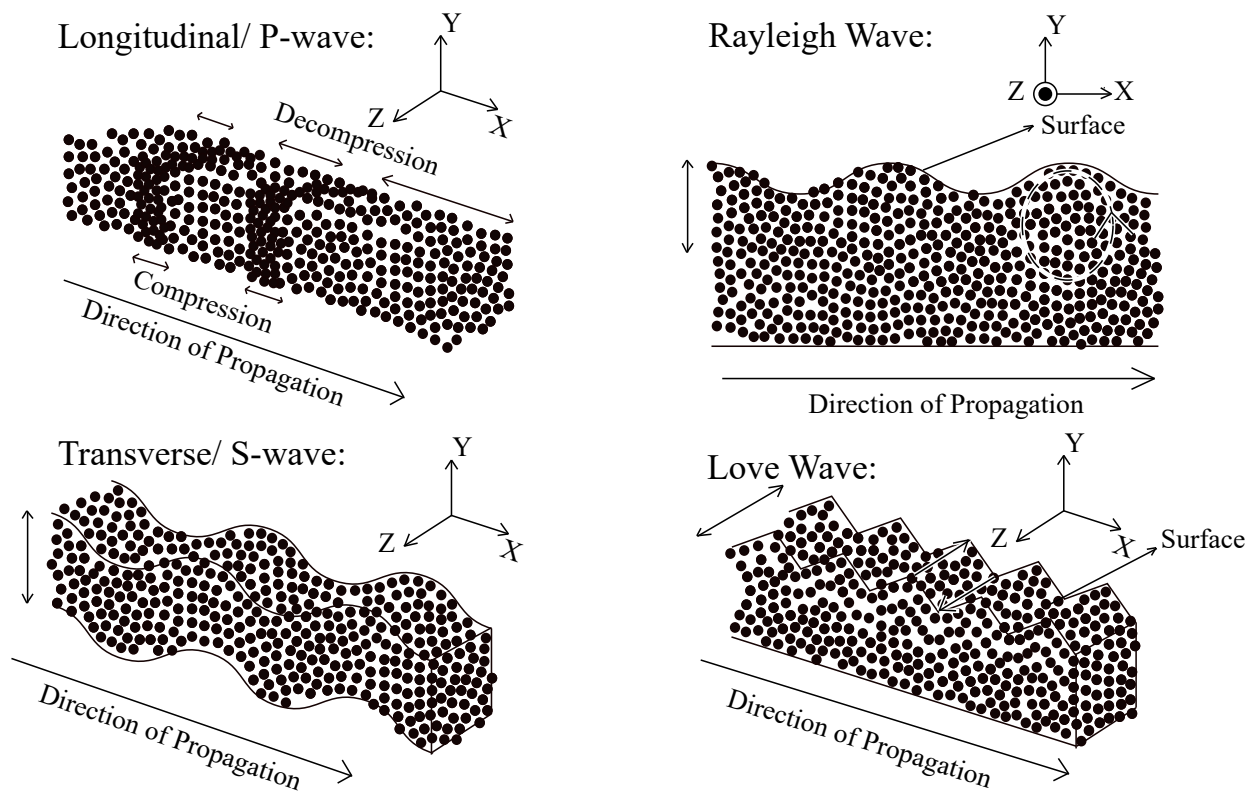

Figure 1.1: Modes of Mechanical Waves. Double ended arrows indicate particle motion inside the media.

Bulk waves are mechanical waves that are present inside media, they are classified into longitudinal waves and transverse/shear waves, referred to as P-waves or S-waves in seismology, respectively. Longitudinal waves manifest 
when the particles of the media are oscillating in the direction of propagation, whereas in case of transverse waves, the particles of the media are oscillating perpendicular to the direction of propagation. On the other hand, surface waves are mechanical waves that are present at the surface or at the interface between different media. Rayleigh waves and Love Waves are different kinds of surface waves. Rayleigh waves have particles moving in elliptical fashion in the plane normal to surface and parallel to the direction of propagation, the amplitude of motion decreases with depth. Love waves are horizontally polarized surface waves, it causes horizontal shifting of the surface with particle motion perpendicular to the direction of propagation. Fig. 1.1 shows pictorial representations of the aforementioned modes of mechanical waves.

Rotational waves are another mode of mechanical waves which have recently caught a lot of attention. They exist due to the media having rotational degrees of freedom $[1,170]$. Cosserat continuum theory takes into account some of the rotational degrees of freedom for the internal heterogeneities of disordered media [39] and has been used for various analyses ranging from localized failure problems [131], dynamics of ballast on railway tracks [189], shear band formation and dissolution [149] due to wave propagation [182, $190,191]$. One of the most striking aspect of "Cosserat behavior" (micropolar rotation of constituent granules) is the existence of rotational sound [121]. It has been extensively studied by experiments and Molecular Dynamics simulations of frictional granular crystals, where characteristic dispersion relations of rotational sound are well predicted by the theory of frictional particles on lattice (Merkel and Luding, 2017 [119], and the references therein). However, how disorder changes the well-established dispersion relations of rotational sound waves is yet to be answered.

\subsection{Signature of Mechanical Wave Propagation in Disordered Media}

A mechanical wave propagating in a disordered medium has a characteristic signature, whether the media is Earth [4] or Moon [43, 133]. The schematic illustration in Fig. 1.2 depicts the signature in time. The longitudinal mode (Pwave) arrives first, then comes the transverse mode (S-wave) and then arrive the Surface waves. Similar signatures of mechanical waves were also observed in laboratory experiments [76, 77, 93, 148, 214, 215]. However, on zooming over a particular mode of a mechanical wave in a time series profile, the me- 


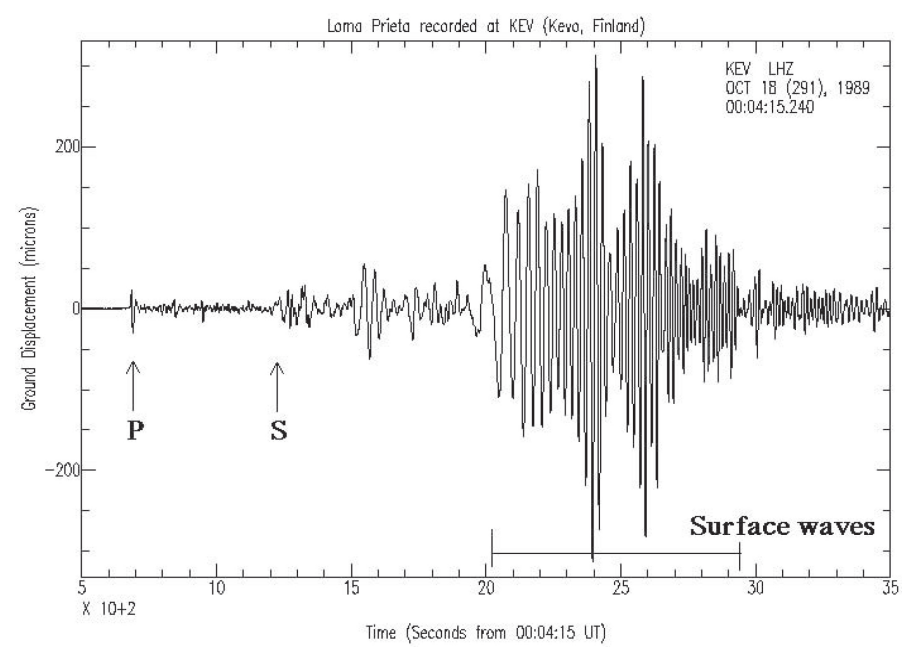

Figure 1.2: Displacement-time profile of an Earthquake (mechanical waves). Figure from: Peter M. Shearer, Introduction to Seismology, 2nd Edition (C) P. Shearer 2009, published by Cambridge University Press [174].

chanical wave signal can be decomposed into two parts (Fig. 1.3), the initial coherent wavefront followed by an incoherent multiply scattered signal also known as "Coda". The initial wavefront is of low frequency in nature, insensitive to the configuration of the media and can withstand configuration based ensemble averaging, hence is coherent in nature. The coherent wavefront is used for determining the bulk sound wave velocities (longitudinal or shear), based on various travel time determination techniques, which are summarized and used in $[8,10,65,81,143,221]$. The Coda is high frequency in nature as it contains waves reflected multiple times e.g. from smaller particles or inclusions (lower size of particles results in lower wavelength of sound and, hence, higher frequency), hence, it contains information about the smaller structures and particles in the media. Due to these characteristics, until the past two decades, Coda was considered as noise and was discarded from signal processing and analyses [211]. However, Coda waves can reveal more properties of the underlying media (smaller particles/constituents resolution) if processed and analyzed correctly by an appropriate model, it can provide additional information and in some cases more than the information provided by the bulk sound wave speed (compressional or shear wave speed). Coda waves have been the foun- 


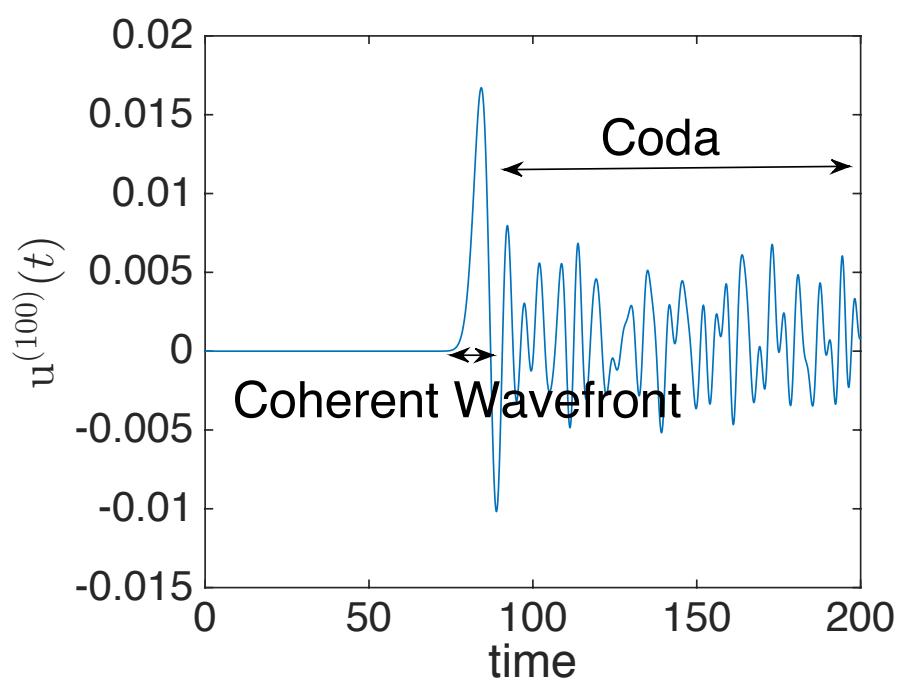

Figure 1.3: Wave propagating in a one-dimensional chain; displacement response of $100^{t h}$ particle; adapted from Chapter 5. See Fig. 5.1 for details.

dation for the development of Coda Wave Interferometry (CWI) which can be used in detection and propagation of cracks in a medium [184].

\subsection{Mesoscopic Wave Phenomena: Diffusion, Co- herent Backscattering Effect \& Weak Localiza- tion}

The diffusive transport of energy in disordered systems is a well known fact. This fact has been readily exploited to develop a diffusion model for propagation of multiply scattered mechanical waves [4, 67, 68, 147, 148, 204, 212]. However, unusual wave phenomena have been observed at intermediate or higher frequencies such as large variations in acoustic wave speed and suppression of wave propagation due to wave localization (Anderson localization [9], similar to the localization observed in ultracold atoms [23, 32, 157], microwave and light [3, 30, 31, 44, 171, 188, 213]). Amongst many definitions of Anderson localizations, two of them rely on vanishing diffusion and decay of spatially lo- 
calized high frequency eigenmodes [36, 97]. If $\psi(r, t)$ is considered as a wave function on the lattice $Z$ with $r$ and $t$ representing space and time, respectively, then $|\psi(r, t)|^{2}$ can be referred to as probability density function of intensity [4, 73]. In a diffusive case

$$
\sum_{r \in Z}|\psi(r, t)|^{2}|r| \approx D \sqrt{t}
$$

where $D$ is the diffusion constant. However, when

$$
\sum_{r \in Z}|\psi(r, t)|^{2}|r| \leq C
$$

then $|\psi(r, t)|^{2}$ is localized and the phenomenon is called Anderson localization.

When the scatterer size and wavelength of wave packets are comparable (intermediate frequencies), the wave spreads out in all directions from the source and undergoes a random multiple scattering process, the constructive interference between two reciprocal wave paths leads to wave localization near the source, this constructive interference between the coherent reciprocal waves is called coherent backscattering $[36,95,196]$, also known as weak localization, with finite probability of the wave returning to the source, leading to enhancement of the local energy density. The concept of weak localization was first discovered in quantum physics 30 years ago [187, 206]. It opposed the idea (which had been agreed upon for a very long time [33, 114, 160, 210]) that multiple scattering destroys wave phenomena, reducing it to radiative transfer, where waves are treated as hard spheres colliding against obstacles (radiative transfer theory $[33,114,160,210])$. The phenomenon of weak localization has been observed in optics $[6,217]$, acoustics $[20,72,200]$ and also in seismics $[94,96]$.

\subsection{Disordered Media: Granular Media}

Disorder or heterogeneity exists at all spatial scales in nature, whether it is soil or matter in space. Classical continuum theory does not consider complexity and heterogeneity as micro-structure present in real materials e.g. porous solids, cracked solids, soil, rocks, composites, polycrystals, biological materials, etc. [1]. Effective Medium Theory (E.M.T) gives the approximate modeling of such media in the large wavelength, low resolution, homogeneous limit, which allows predicting some macroscopic/material properties [15, 48, 51]. Granular media are such a disordered media, a conglomerate of discrete and 
solid particles/grains. Examples can be found often in our daily life e.g. sugar, cereals, powders, tablets or materials in nature such as sand, snow, etc. As quoted in [170]: "granular systems are a useful starting point in the description of ocean sediments and sedimentary rocks". In the upcoming subsections, modeling of granular media for sound propagation analyses, the inconsistencies of some previously used models, the backbone of sound propagation in granular media (force chains) and finally experimental investigations related to this media will be discussed.

\subsubsection{Modeling for Structure and Dynamic Analyses}

As mentioned previously, E.M.T and its derivative modified theories have been used for modeling sound propagation in granular media [110, 140, 208]. However, the shortcomings of E.M.T have been highlighted in $[111,146]$ (because of disorder, nonlinearity and the non-affine nature of granular materials), especially in the intermediate or high frequency regimes of mechanical waves.

The Discrete Element Method (D.E.M) [42], a numerical modeling technique for each of many single particles, has been applied to sound propagation through granular assemblies [100, 128, 140, 141, 143, 195]; it has been quite successful in resolving intermediate frequencies of mechanical waves and revealing their respective characteristics, which were later confirmed with experiments (Sec. 1.6.2), such as Dispersion [89, 90], Attenuation (Sec. 1.2), Anderson localization [87], etc. However, numerical modeling can be cumbersome if the system size is large (resulting in high computational costs). Hence, a bridge is needed, which retains the resolution capacity of the model (takes into account the micro-structure of a material) and is also valid for the biggest system sizes (Sec. 1.7).

\subsubsection{Experimental Investigations}

Earthquakes are naturally occurring mechanical waves; they have been measured and recorded since a very long time; they have been quintessential in determining the internal structure of Earth, hence, their study is one of the early experimental investigations of mechanical waves in soil [55, 174].

Ultrasound experiments have become instrumental in recent years to study the unique vibrational response of granular materials [102, 103, 104, 147]. For $\mathrm{kHz}-\mathrm{MHz}$ frequency signals (mechanical waves) that are sent through materials, the received signals are analyzed for identifying the internal structure of 
the media; they have the ability to detect full wave field and not only just the intensity. They assist in performing experiments both resolved in space and time [76, 78, 80, 216]. Anderson localization after being observed experimentally in electronics and optics was observed in the case of classical waves with the aid of ultrasonic experiments [72]. Ultrasonic experiments have also been used for geotechnical investigations to determine material parameters like bulk/shear stiffness $[115,140,141,142,143]$. Stiffness measured at small strain levels is assumed to be elastic stiffness, but, it is a difficult parameter to determine due to possible non-linearity [11]. The small amplitude stress and strain are measured by LVDTs (Linear Variable Differential Transformers), which can measure strain up to $0.0001 \%$, introduced in Ref. [41]. Geotechnical investigations nowadays employ bender elements. Bender elements are piezoelectric devices which deflect when electrical current passes through them or produce a voltage difference when deflected by a mechanical wave. Bender elements are interesting, since they can emit and receive shear waves and hence aid in directly measuring the shear stiffness of soil $[54,176]$.

Granular/Force Chains In a granular material, grain-grain forces are correlated in a line-like fashion called force chains [186]. Force chains indicate the direction of major principle stress/fabric when the sample is anisotropically loaded, forming links of contacting particles that behave like "columns" in the sample. Force chains act as pathways or backbone for sound to propagate. Practically the only possibility to measure such stresses and forces is photoelasticity, using changes in the optical properties of a material under mechanical deformation [38]. Photoelasticity thus has been crucial in understanding the relationship between stiffness and contact force chain networks of discrete disordered samples [18, 19, 21, 101, 153, 155]. It can be used to measure both normal and tangential forces of photoelastic disks/particles (particles that brighten under compression when viewed between polaroid screens) from isochromatic fringes [107, 108, 109]. Experiments with photoelastic disks/particles assists in understanding the dynamics of sound wave propagation across force chains $[146,226]$, showing that the wave travels faster in the direction of increased loading [164]. In addition to helping understand wave propagation and force chain networks, photoelasticity has also been helpful to model and visualize seismogenic faults [45] and understanding the jamming transition in granular systems [153, 154]. 


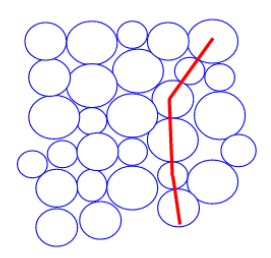

(a) A granular/force chain from a network (schematic). Adapted from Chapter 2.

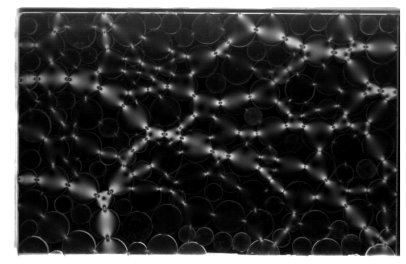

(b) Photoelastic disks exhibiting force chain networks. Adapted from Chapter 5.

Figure 1.4: Force chains: backbone of sound propagation in grnaular materials.

\subsection{Stochastic Modeling}

Propagating mechanical waves in a medium are sensitive to the heterogeneity of the medium as their characteristics (e.g. frequency, amplitude, etc.) change after encountering a heterogeneity [97, 128, 222]. This sensitivity is often exploited to reveal the details of its source and the wave path/media by modeling it in terms of mathematical equations with the relevant physics and determining the parameters of this physical model. A deterministic interpretation is not always possible because of extreme sensitivity of these waves to the details (multiple interference of waves from heterogeneities) leading to misrepresentation or misinterpretation of information (signals). Numerical techniques like Finite Difference Method (F.D.M), Finite Element Method (F.E.M), Discrete Element Method (D.E.M), etc. are used for modeling wave propagation through materials [83, 86, 132, 141, 194], however, as mentioned previously, determining the parameters of these models sometimes becomes non-feasible because of either very large system size or very complex micro-structure (heterogeneity). In such cases, a statistical treatment of the heterogeneities in the media can assist in overcoming some of these difficulties [4, 35, 47, 75].

Thinking on these lines leads to the proposition of developing stochastic models for various phenomena (dispersion, scattering attenuation, etc.) occurring during wave propagation (Chapter 2, 3, 4 and 5). Equations/relations are developed on the basis of discrete elements/particles while modeling internal heterogeneity of the medium as opposed to a continuous body in the following chapters, hence, these equations can be a development or improvement over previous continuum models for particulate media like soil. To isolate different modes of mechanical waves, 1D and 2D discrete element numerical schemes 
are used, e.g. 1D chain of spheres are used to isolate longitudinal/P-wave mode (Chapter-2, 3, 5) and 2D spherical disks are used for studying rotational mode (Chapter-4).

\subsection{Nonlinear Phenomena}

The dynamics of unconsolidated granular materials exhibit numerous nonlinear phenomena. A detailed analyses has been presented in [202]. The challenge has always been to understand, predict and model them. One of the nonlinear phenomena is the $v \propto p^{1 / 4}$ ( $p$ implies pressure) dependence of the wave velocity $(v)$ at low confining pressures; unlike the high pressure regime when $v \propto p^{1 / 6}$ is observed, as predicted by Hertz-Mindlin [62, 110, 173, 186]. It occurs either because of formation of conical contacts due to surface asperities of the particles or due to formation of new contacts $[118,140]$. Another nonlinear phenomenon exhibited by the granular materials is the self-demodulation effect [201], the effect is the generation of a difference frequency waveform ( $\Omega=\omega_{1}-\omega_{2}$ ) when $\omega_{1}$ and $\omega_{2}$ frequency waveforms are present in the material $\left(\omega_{1}>\omega_{2}\right)$; the main highlight of this effect is the longer propagation of lower frequency wave $\Omega$ as the higher frequencies $\omega_{1}$ and $\omega_{2}$ get attenuated, the effect was observed experimentally in [134, 139, 227]. Moreover, granular materials exhibit solitary wave propagation, another typical nonlinear phenomenon. It manifests under conditions of non-linear contact forces or complete lack of force (sonic vacuum with opening and closing of contacts) between particles of the particulate media [113, 135, 136, 192]; under these nonlinear conditions, propagating localized wave packets are observed, termed as "breathers" [58, 74, $125,172]$; these breathers are also responsible for the destruction of traditional localization [87] as they siphon the energy from the localized energy sites.

The modeling of the granular media and the analysis of these non-linear phenomena have assisted researchers in designing materials for many applications e.g. shock protection, energy trapping/focusing, etc. Granular chains have been instrumental in giving insights for designing metamaterials because of their simple most one dimensional structure. Tapered and decorated granular chains have been modeled both computationally and experimentally for potential applications as metamaterials $[26,27,50,57,70,112,158]$. 


\subsection{Thesis Outline}

- Chapter-1 introduces the terminologies, definitions, past and ongoing research work related to mechanical wave propagation in solid, static and disordered granular materials.

- Chapter-2 is about the effect of mass disorder on the sound wave velocity, studied with the aid of a mass disordered one-dimensional granular chain. The total energy associated with the eigenmodes is used to define dispersion relations for disordered chains [177].

- Chapter-3 focuses on the transfer of Energy with distance as well as across different wavenumbers, as the mechanical wave propagates. The diffusive characteristic of energy propagation has been discussed. A Master Equation is devised and utilized for analyzing the transfer of energy across different wavenumbers, studied with the aid of a one-dimensional granular chain [178].

- Chapter-4 is about Rotational sound propagation in disordered frictional disk systems studied with the aid of two dimensional discrete element simulations. The dispersion relations associated with rotational modes are explained by a modified lattice model.

- Chapter-5 contains nonlinear (Hertzian) numerical simulations of a coherent wave propagating along a one dimensional chain of particles, supplemented by one and two dimensional photoelastic experimental investigations [179].

- Chapter-6 summarizes and concludes the investigations carried out in the thesis with proposals for future work. 



\section{Chapter 2}

\section{Effect of Mass Disorder on Bulk Sound Wave Speed: A Multiscale Spectral Analysis}

Natural order is disorder.

Zaheer (Avatar: Legend of Korra)

Disorder of size (polydispersity) and mass of discrete elements/particles in randomly structured media (e.g. granular matter like soil) has numerous effects on the materials' sound propagation characteristics. The influence of disorder on the sound wave speed and its low pass frequency filtering characteristics is the subject of this study. Goal is understanding the connection between the particle-microscale disorder and dynamics and the system-macro-scale wave propagation which can be applied to non-destructive testing, seismic exploration of buried objects (oil, mineral, etc.) or to study the internal structure of the Earth. To isolate the longitudinal P-wave mode from shear and rotational modes, a one-dimensional system of elements/particles is used to study the effect of mass disorder alone via (direct and/or ensemble averaged) real time signals, signals in Fourier space and dispersion curves. Increase in mass disorder (where disorder has been defined such that it is independent of the shape of the probability distribution of masses) decreases the sound wave speed along a granular chain. Energies associated with the eigenmodes are conserved, independent of time, and have been used to derive dispersion 
relations for disordered chains; these dispersion relations confirm the decrease in cut-off frequency and thus wave speed with increasing disorder. ${ }^{1}$

\subsection{Introduction}

Sound wave propagation through matter has been an extensive area of research (as textbook example, see [5]) whether it is applied for the study of earthquakes or the internal structure of the Earth, as well as oil, gas or mineral exploration (seismology). Waves can be used for dissecting the human body without using blades, revealing material properties through non-destructive testing (ultrasonics), studying the structure of lattices or designing metamaterials. There are numerous applications and uncountable problems which still need to be solved, where the challenge has always been resolving the finest structures of matter using wave propagation and hence, steps are being taken in the direction of micromechanics of seismic wave propagation, see e.g. [141].

Disordered/Heterogeneous/Random media cause multiple scattering of seismic waves, mechanisms which eventually cause them to become dispersed, attenuated and localized in space ([165], [167]). The phenomenon of multiple scattering causes the formation of the so called "coda" which is the tail of a propagating wave pulse. While coda was earlier treated as noise ([211]), now it has given way to coda wave interferometry with multiple applications ([184]). The coda has been studied in detail in laboratory experiments with uniaxial or triaxial devices, for e.g., pulse propagation across glass beads ([77]), sintered glass beads ([67]), indicating extreme sensitivity towards system preparation and configuration and getting washed out on ensemble averaging with only the coherent part of the signal remaining. In [14], it was shown that macroscopic/seismic waves governed by the classical wave equation did not exhibit localization at lower frequencies but, this idea got repudiated by [96], where weak localization (a mesoscopic phenomenon, precursor to wave localization; [175]) was experimentally observed at frequencies as low as $20 \mathrm{~Hz}$, indicating the inadequacy of the classical wave equation.

In recent years, wave propagation through granular materials has attracted a lot of attention. Granular material is a heterogeneous media with many discretized units and can be used for modeling geometrically heterogeneous media ([117]). The studies done using ordered/disordered lattices for wave propagation ([61], [40], etc.) has helped to understand wave propagation in granular

\footnotetext{
${ }^{1}$ Published as: Shrivastava, R.K., \& Luding, S. (2017). Effect of disorder on bulk sound wave speed: a multiscale spectral analysis. Nonlinear Processes in Geophysics, 24(3), 435.
} 
materials through dispersion relations, frequency filtering, etc. Scaling laws allow to relate various physical parameters like density, pressure, coordination number, etc., with the moduli, forming an Effective Medium Theory (EMT) for granular matter ([110]).

Nesterenko [136] showed the existence of localized wave packets propagating in a non-linear granular chain (one dimensional granular material) under the condition of "sonic vacuum" (in the limit of zero acoustic wave speed and vanishing confining pressure) thus forming supersonic solitary waves; such concepts have been exploited immensely to develop various kinds of metamaterials like for shock and energy trapping ([46]), an acoustic diode ([25]) or for understanding and studying jamming transitions in granular matter ([214], [207]). Some of the open questions and developments related to wave propagation in unconsolidated granular matter, like higher harmonics generation, non-linear multiple scattering, soft modes, rotational modes, etc., have been addressed by [202]. However, in the following, the focus of attention will not be on solitons and unconsolidated granular matter, hence, there will be no occurrence of sonic vacuum during analyses (no opening and closing of contacts of particles).

A striking characteristic of consolidated granular matter is that grain-grain forces are arranged and correlated in a linear manner known as force chains ([186]). Similar to the force chains, [149] showed the existence of moment chains in granular media, i.e. correlations of grain-grain mutual rotations. These chains are mesoscopic structures and are just one of the many microrotational effects of granules. Cosserat continuum theory can be used to model these micropolar/micro-rotational effects, as discussed in detail by [151].

The force chains/granular chains which carry the large forces of the system supposedly support faster sound transmission across granular matter ([145]). In [146], it was seen from experiments with 2-dimensional photo-elastic disks that vibration propagates along the granular chains, visualized by the brightness due to compression between the particles; however, the exact mechanisms of propagation of the vibrations are still a matter of ongoing research. Our system under investigation will be a single one of such granular chains (Fig. 2.1); it will assist in isolating the P-wave or the longitudinal excitation from all other kinds of excitations (S-wave, rotational wave, etc). In [120] it was seen that inclusion or removal of rotation does not significantly affect the longitudinal mode in an ordered granular crystal. However, the situation is different when rotations become prominent and other wave modes cannot be ignored (see [223], Merkel and Luding (2016) and the references therein).

Even though very simplistic, a polydisperse granular chain can have two kinds of disorder, mass disorder and stiffness disorder ([97]), the mass disorder 
has much stronger contribution towards disorder than stiffness because mass $\propto$ radius $^{3}$ whereas, stiffness $\propto$ radius $^{1 / 3}$ ([2]). Hence, only mass disorder for the disordered granular chain has been chosen. However, there are processes when stiffness disorder cannot be ignored, for instance, the processes when the repulsive interaction force between the fragments/elements of the material being modeled has different stiffness during compression and tension (bilinear oscillator; [53]), infinite stiffness while compression (impact oscillator; [52] and [69]) or negative stiffness ([150] and [56]).

In Sect. 2.2 an impulse propagating across a granular chain is modeled. A similar model was used in [115] and [97]. Section 2.2.8 concerns the dispersion relation for wave propagation across a granular medium, Sect. 2.2.9 concerns the group velocity and Sect. 2.2.10 concerns a novel way of computing the dispersion relation in terms of moments of eigenmodal energy. In Sect. 2.3, the equations mentioned in Sect. 2.2 are computed numerically and the observations are discussed. Sect. 2.4 summarizes and concludes the observations made in Sect. 2.3 with Sect. 2.2 as the foundation and an outlook of the ongoing as well as possible future research work on wave propagation in granular matter is given.

\subsection{Modeling a general one-dimensional chain}

A one-dimensional chain of $N+2$ particles is considered. Each particle $i$ has mass $\tilde{m}^{(i)}$ and contact stiffness $\tilde{\kappa}_{(i, j)}$ with respect to a neighboring particle $j$. The tilde symbols are used for dimensional quantities. The interaction force
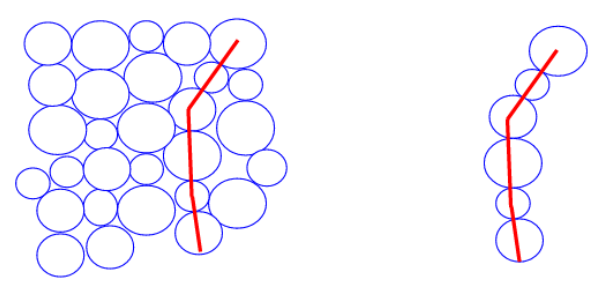

Figure 2.1: A granular/force chain from a network (schematic). 
experienced by neighboring particles $i$ and $j$ is

$$
\tilde{F}_{(i, j)}=\tilde{\kappa}_{(i, j)} \tilde{\delta}_{(i, j)}^{1+\beta}, \quad \tilde{\delta}_{(i, j)} \geq 0,
$$

with the contact stiffness $\tilde{\kappa}_{(i, j)}$ and the particle overlap $\tilde{\delta}_{(i, j)}=\tilde{r}^{(i)}+\tilde{r}^{(j)}-\mid \tilde{x}^{(j)}-$ $\tilde{x}^{(i)} \mid$, with the radius $\tilde{r}$ and co-ordinates $\tilde{x}$ of the centers of the particles. The Hertzian and linear model are given by $\beta=1 / 2$ and $\beta=0$, respectively ([97]). This force resembles the framework of the Discrete Element Method where the overlap of particles substitutes their deformations at the contacts, which would be much more difficult and time consuming to resolve with a finite element model of deformable bodies. Assume that the chain is pre-compressed by an external applied force $\tilde{F}_{o}$, the characteristic overlap of the particles in static equilibrium $\left(\tilde{\Delta}_{o}\right)$ when all the contact stiffness $\left(\tilde{\kappa}_{(i, j)}\right)$ of particles are chosen as

At rest :

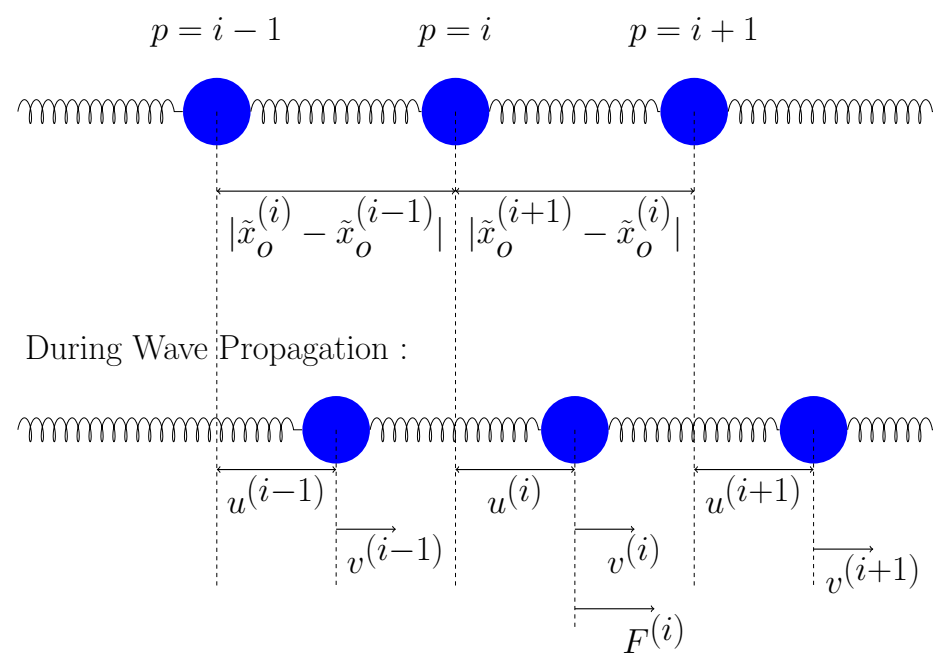

Figure 2.2: Chain of granular elements during dynamic wave propagation with length scaled by the characteristic equilibrium distance $\tilde{\Delta}_{o},\left|\tilde{x}_{o}^{(i)}-\tilde{x}_{o}^{(i-1)}\right|=\mid\left(\tilde{r}^{(i)}+\right.$ $\left.\tilde{r}^{(i-1)}\right)-\tilde{\Delta}_{(i, i-1)} \mid$ 
$\tilde{\kappa}_{o}$ (characteristic contact stiffness) is thus defined as

$$
\tilde{\Delta}_{o}=\left(\frac{\tilde{F}_{o}}{\tilde{\kappa}_{o}}\right)^{1 /(1+\beta)},
$$

where the unit of $\tilde{\kappa}$ depends on $\beta$.

\subsubsection{Non-dimensionalization}

A length scale $\tilde{\ell}$ can be chosen such that the scaled particle overlap $\delta_{(i, j)}=$ $\tilde{\delta}_{(i, j)} / \tilde{\ell}$ yields

$$
\tilde{F}_{(i, j)}=\tilde{\kappa}_{(i, j)} \tilde{\ell}^{1+\beta} \delta_{(i, j)}^{1+\beta}
$$

There are several length scales $\tilde{\ell}$ that can be chosen, e.g. the particle size, the driving amplitude or the initial overlap

$$
\Delta_{(i, j)}=\left(\frac{\tilde{F}_{o}}{\tilde{\kappa}_{(i, j)} \tilde{\ell}^{1+\beta}}\right)^{1 /(1+\beta)} .
$$

of the particles in static equilibrium. The latter is chosen for computations here so that $\Delta_{o} \equiv 1$ if all $\tilde{\kappa}_{(i, j)}=\tilde{\kappa}_{o}$. Other dimensionless quantities are, the mass $b=\tilde{m} / \tilde{M}_{1}$ where $\tilde{M}_{1}$ is the first moment of the mass distribution of the particles of the media, as shown in Appendix $C$ (the unscaled average mass of the particles), the dimensionless displacement $u=\tilde{u} / \tilde{l}$ and the dimensionless spring constant $\kappa=\tilde{\kappa} / \tilde{\kappa}_{o}$, the characteristic time scale becomes

$$
\tilde{t}_{c}=\sqrt{\frac{\tilde{M}_{1}}{\tilde{\kappa}_{o} \tilde{\ell}^{\beta}}},
$$

which gives us the dimensionless time $t=\tilde{t} / \tilde{t}_{c}$. The displacement of particle $i$ from its equilibrium position $\tilde{x}_{o}^{(i)}$ is $\tilde{u}^{(i)}=\tilde{\ell} u^{(i)}=\tilde{x}^{(i)}-\tilde{x}_{o}^{(i)}$, so that the overlap becomes, $\delta_{(i, j)}=\Delta_{(i, j)}-\left(u^{(j)}-u^{(i)}\right)$. Finally, the interaction forces scale as

$$
F_{(i, j)}=\frac{\tilde{t}_{c}^{2}}{\tilde{M}_{1} \tilde{\ell}} F_{(i, j)} .
$$




\subsubsection{Equation of Motion : Nonlinear (Hertzian)}

The equation of motion for any particle $i$ (except the boundary particles at either end of the chain) by using Eq. (2.3), (2.4) and non-dimensionalization (Sect. 2.2.1) can be written as

$$
b^{(i)} \frac{\mathrm{d}^{2} u^{(i)}}{\mathrm{d} t^{2}}=F_{(i-1, i)}+F_{(i, i+1)}=\kappa_{(i-1, i)} \delta_{(i-1, i)}^{1+\beta}-\kappa_{(i, i+1)} \delta_{(i, i+1)}^{1+\beta},
$$

which can also be written as

$$
\begin{aligned}
b^{(i)} \frac{\mathrm{d}^{2} u^{(i)}}{\mathrm{d} t^{2}} & =\kappa_{(i-1, i)}\left[\Delta_{(i-1, i)}-\left(u^{(i)}-u^{(i-1)}\right)\right]^{(1+\beta)} \\
& -\kappa_{(i+1, i)}\left[\Delta_{(i+1, i)}-\left(u^{(i+1)}-u^{(i)}\right)\right]^{(1+\beta)} .
\end{aligned}
$$

For particles interacting repulsively with Hertzian potential, $\beta=1 / 2$, Eq. (2.7) or (3.5) can be solved numerically.

\subsubsection{Equation of Motion : Linear}

The repulsive interaction force can be expressed as a power series and can be expanded about the initial overlap $\Delta_{(i, j)}$ due to pre-compression,

$$
F_{(i, j)}=\kappa_{(i, j)} \Delta_{(i, j)}^{1+\beta}+\kappa_{(i, j)}(1+\beta) \Delta_{(i, j)}^{\beta}\left(\delta_{(i, j)}-\Delta_{(i, j)}\right)+\frac{1}{2} \kappa_{(i, j)} \beta(1+\beta) \Delta_{(i, j)}^{\beta-1}\left(\delta_{(i, j)}-\Delta_{(i, j)}\right)^{2}+\ldots
$$

For small displacements from the equilibrium condition (during wave propagation), using the definition of $\delta_{(i, j)}$ and after ignoring higher order non-linear terms, we arrive at

$$
F_{(i, j)}=\kappa_{(i, j)} \Delta_{(i, j)}^{1+\beta}-\kappa_{(i, j)}(1+\beta) \Delta_{(i, j)}^{\beta}\left(u^{(j)}-u^{(i)}\right) .
$$

Inserting the force relation (Eq. (5.8)) in Eq. (2.7), we get the general, linearized equation of motion:

$$
\begin{aligned}
b^{(i)} \frac{\mathrm{d}^{2} u^{(i)}}{\mathrm{d} t^{2}} & =\kappa_{(i-1, i)} \Delta_{(i-1, i)}^{\beta}\left[\Delta_{(i-1, i)}-(1+\beta)\left(u^{(i)}-u^{(i-1)}\right)\right] \\
& -\kappa_{(i+1, i)} \Delta_{(i, i+1)}^{\beta}\left[\Delta_{(i+1, i)}-(1+\beta)\left(u^{(i+1)}-u^{(i)}\right)\right] .
\end{aligned}
$$


For Hertzian nonlinear repulsive interaction force between the particles, the scaled stiffness $\kappa_{(i, j)}$ and initial overlap $\Delta_{(i, j)}$ are given as follows (see Appendix $B$ for details):

$$
\kappa_{(i, j)}=\sqrt{\frac{2}{b^{(i) 1 / 3}+b^{(j) 1 / 3}}}\left(b^{(i)} b^{(j)}\right)^{1 / 6},
$$

and

$$
\Delta_{(i, j)}=\kappa_{(i, j)}^{(-2 / 3)} .
$$

Eq. (5.9) can be written in a linearized form as

$$
\frac{b^{(i)}}{(1+\beta)} \frac{\mathrm{d}^{2} u^{(i)}}{\mathrm{d} t^{2}}=\kappa_{(i+1, i)}^{1 /(1+\beta)}\left(u^{(i+1)}-u^{(i)}\right)-\kappa_{(i-1, i)}^{1 /(1+\beta)}\left(u^{(i)}-u^{(i-1)}\right)
$$

Since, we are interested only in mass disorder, we can choose all coupling stiffness $\left(\kappa_{(i, j)}\right)$ as 1 . Now, Eq. (2.14) for individual particles can be written as

$$
\frac{b^{(i)}}{(1+\beta)} \frac{\mathrm{d}^{2} u^{(i)}}{\mathrm{d} t^{2}}=u^{(i+1)}-2 u^{(i)}+u^{(i-1)}
$$

The factor $\frac{1}{1+\beta}$ becomes 1 for the linear contact model $(\beta=0)$ and it becomes $2 / 3$ for the Hertzian contact model $(\beta=1 / 2)$. It can be observed that the factor $\frac{1}{1+\beta}$ has only multiplicative influence on the physical parameters. Since, in our system of equations (Eq. (2.15)) only mass disorder is present, the masses of the particles get multiplied by this factor $\left(\frac{1}{1+\beta}\right)$. For further analysis, $\beta=0$ has been chosen so that

$$
b^{(i)} \frac{\mathrm{d}^{2} u^{(i)}}{\mathrm{d} t^{2}}=u^{(i+1)}-2 u^{(i)}+u^{(i-1)}
$$

This results in $N$ equations which eventually can be expressed in matrix form:

$$
\mathbf{M} \frac{\mathrm{d}^{2} \mathbf{u}}{\mathrm{d} t^{2}}=\mathbf{K u}+\mathbf{f},
$$

where $\mathbf{M}$ is a diagonal mass matrix with entries $b^{(1)}, b^{(2)}, b^{(3)}, \ldots, b^{(N)}$ and zero otherwise; $\mathbf{K}$ is a matrix with diagonal entries $-\left(\kappa_{(i+1, i)}+\kappa_{(i-1, i)}\right)=-2$, superdiagonal $\left(\kappa_{(i+1, i)}\right)$ and subdiagonal $\left(\kappa_{(i-1, i)}\right)$ entries +1 and zero otherwise for $\kappa=1$. $\mathbf{f}$ is the external force which depends on the specified driving. Introducing, $\mathbf{A}=-\mathbf{M}^{-1} \mathbf{K}$ then, Eq. (2.17) can be written as

$$
-\frac{\mathrm{d}^{2} \mathbf{u}}{\mathrm{d} t^{2}}=\mathbf{A u}-\mathbf{M}^{-1} \mathbf{f} \text {. }
$$




\subsubsection{Analysis in real space/spatial Fourier space :}

Using an ansatz for real space and another ansatz for spatial Fourier space in Eq. (2.18) (the calligraphic fonts from now onwards will depict the spatial Fourier transform counterparts of the real space parameters),

$$
\mathbf{u}=\mathbf{u}^{\prime} e^{i \omega t} \quad \text { or } \quad \mathscr{U}=\mathscr{U}^{\prime} e^{i(\omega t-k u)},
$$

one has

$$
\mathbf{A u}=\omega^{2} \mathbf{u} \text { or } \quad \mathbf{A} \mathscr{U}=\omega^{2} \mathscr{U},
$$

where $k$ is the wavenumber and $\mathscr{U}=\int_{-\infty}^{\infty} \int_{-\infty}^{\infty} u e^{-i(\omega t-k u)} \mathrm{d} t \mathrm{~d} u$ is the double Fourier transform (spatial as well as temporal) ansatz. Equation (2.20) is a familiar eigen value problem. The eigenvalues $\omega_{j}^{2}$ and eigenvectors $\mathbf{s}_{(j)}$ of the matrix $\mathbf{A}$ give the eigendomain of the granular chain that are independent of the external driving. The square roots of the eigenvalues, $\omega_{j}$, are the natural frequencies of the chain. The set of eigenvectors can be orthonormalised to obey the orthonormality condition:

$$
\mathbf{s}_{(i)}^{\mathrm{T}} \mathbf{M} \mathbf{s}_{(j)}=\delta_{i j},
$$

with $\delta_{i j}$ being the Kronecker delta symbol. The $\mathbf{S}$ matrix or the eigenbasis matrix can be constructed with $\mathbf{s}_{(j)}$ as the columns of the matrix, which can be used to transform back and forth from real domain to eigen domain. The columns $\left(\mathbf{s}_{(j)}\right)$ of the matrix $\mathbf{S}$ are sorted such that the corresponding eigenvalues $\omega_{j}$ are in increasing order. The vector of eigenmode amplitudes is

$$
\mathbf{z}=\mathbf{S}^{-1} \mathbf{u} \text { or } \mathcal{Z}=\mathbf{S}^{-1} \mathscr{U} \text {. }
$$

A matrix $\mathbf{G}$ consisting of eigenvalues $\omega_{j}^{2}$ along the diagonal (in increasing order) is formulated such that $\mathbf{G}=\mathbf{S}^{-1} \mathbf{A S}$ which allows the transformation of Eq. (2.17) into the eigendomain as

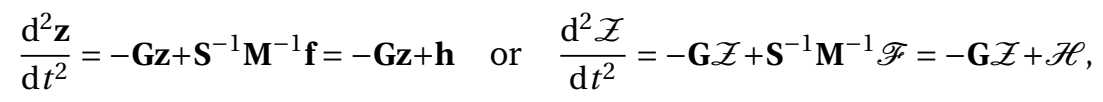

which defines $h$ and $\mathscr{H}$ implicitly. The differential equations (2.23) are decoupled and can be solved to give

$$
\mathbf{z}(t)=\mathbf{C}^{(1)} \mathbf{a}+\mathbf{C}^{(2)} \mathbf{b}+\mathbf{z}_{P}(t) \quad \text { or } \quad \mathcal{Z}(t)=\mathbf{C}^{(1)} \mathscr{A}+\mathbf{C}^{(2)} \mathscr{B}+Z_{P}(t)
$$


where $\mathbf{C}^{(1)}$ is a diagonal matrix with $\mathbf{C}_{j, j}^{(1)}=\sin \left(\omega_{j} t\right), \mathbf{C}^{(2)}$ is a diagonal matrix with $\mathbf{C}_{j, j}^{(2)}=\cos \left(\omega_{j} t\right)$, and $\mathbf{z}_{P}(t)$ or $Z_{P}(t)$ are the particular solutions of the differential equations, which depend on $\mathbf{h}$ or $\mathscr{H}$ and, hence, depend on the external driving force $\mathbf{f}$ or $\mathscr{F}$. The vectors $\mathbf{a}$ or $\mathscr{A}$ and $\mathbf{b}$ or $\mathscr{B}$ are determined by the initial conditions from the initial displacement $\left(\mathbf{u}_{o}\right.$ or $\left.\mathscr{U}_{o}(k)\right)$ and velocities $\left(\mathbf{v}_{o}\right.$ or $\left.V_{o}(k)\right)$.

$$
\mathbf{b}=\mathbf{S}^{-1} \mathbf{u}_{o}-\mathbf{z}_{P}(0) \quad \text { or } \quad \mathscr{B}=\mathbf{S}^{-1} \mathbf{u}_{o}-\mathbf{z}_{P}(0)
$$

and

$$
\mathbf{a}=\mathbf{H}^{-1} \mathbf{S}^{-1} \mathbf{v}_{o}-\left.\mathbf{H}^{-1} \frac{\mathrm{d} \mathbf{z}_{P}(t)}{\mathrm{d} t}\right|_{t=0} \quad \text { or } \quad \mathscr{A}=\mathbf{H}^{-1} \mathbf{S}^{-1} \mathcal{V}_{o}-\left.\mathbf{H}^{-1} \frac{\mathrm{d} Z_{P}(t)}{\mathrm{d} t}\right|_{t=0},
$$

where $\mathbf{H}$ is a diagonal matrix with $\omega_{j}$ as the diagonal elements. $\mathbf{a}$ and $\mathbf{b}$ or $\mathscr{A}$ and $\mathscr{B}$ are column vectors with column elements $a_{j}$ and $b_{j}$ or $\mathscr{A}_{j}$ and $\mathscr{B}_{j}$, associated with a particular eigenfrequency $\left(\omega_{j}\right)$. The solution in real space can be obtained by the transformation mentioned in Eq. (2.22) which can be applied on Eq. (2.24) to give

$$
\mathbf{u}(t)=\mathbf{S C}^{(1)} \mathbf{a}+\mathbf{S C}^{(2)} \mathbf{b}+\mathbf{u}_{P}(t) \quad \text { or } \quad \mathscr{U}(t)=\mathbf{S C}^{(1)} \mathscr{A}+\mathbf{S C}^{(2)} \mathscr{B}+\mathscr{U}_{P}(t) .
$$

\subsubsection{Initial Conditions : Impulse Driving}

The initial conditions required to solve various special cases are the initial displacements $\left(\mathbf{u}_{o}\right)$ and initial velocities $\left(\mathbf{v}_{o}\right)$ in real space and $V_{o}$ and $\mathscr{U}_{o}$ in spatial Fourier space. Besides the sinus driving used in [97], we apply impulse driving initial condition. For an impulse driving mode, the boundary conditions are as follows:

$$
u^{(i)}(t=0)=0, v^{(i \neq 1)}(t=0)=0, v^{(1)}(t=0)=v_{o} .
$$

An impulse driven chain has an impulse imparted to the first particle, $i=1$ with initial velocity $v_{o}$. Since the focus of our study is not on the occurrence of sonic vacuum ([136]), the initial impulse $\left(v_{o}\right)$ should be chosen small enough to avoid opening of contacts. Using Eq. (2.27), (2.25), (2.26) and the initial conditions for the impulse driven chain i.e. $\mathbf{f}=0$ (no driving present), $\mathbf{u}_{o}=0$ and $\mathbf{v}_{o}=\left[\begin{array}{lll}v_{o} & 0 & \ldots .0\end{array}\right]^{\mathrm{T}}$, we get

$$
\mathbf{a}=\mathbf{H}^{-1} \mathbf{S}^{-1} \mathbf{v}_{o}, \quad \mathbf{b}=0,
$$


and

$$
\mathbf{u}=\mathbf{S} \mathbf{C}^{(1)} \mathbf{H}^{-1} \mathbf{S}^{-1} \mathbf{v}_{\boldsymbol{o}} \quad \& \quad \mathbf{v}=\mathbf{S C}^{(2)} \mathbf{S}^{-1} \mathbf{v}_{o}
$$

which implies that displacements and velocities of all particles $p$ are given analytically by

$$
u^{(p)}(t)=v_{o} \sum_{j=1}^{N} \frac{S_{p j} S_{1 j} \sin \left(\omega_{(j)} t\right)}{\omega_{(j)}} \quad \& \quad v^{(p)}(t)=v_{o} \sum_{j=1}^{N} S_{p j} S_{1 j} \cos \left(\omega_{(j)} t\right) .
$$

In wavenumber space (spatial Fourier transform), the initial condition is specified by $V_{o}(k)$ which can be a sine or cosine function in terms of wavenumber $(k)$. Using Eq. (2.27) and $V_{o}(k)$, we get

$$
\mathscr{A}=\mathbf{H}^{-1} \mathbf{S}^{-1} \mathcal{V}_{o}(k) \quad, \quad \mathscr{B}=0,
$$

and thus,

$$
\mathscr{U}=\mathbf{S C}^{(1)} \mathscr{A} \quad \& \quad V=\mathbf{S C}^{(2)} \mathbf{H} \mathscr{A},
$$

\subsubsection{Mass Distribution, Disorder Parameter $(\xi)$, Ensemble Averaging \& Binning}

The mass distribution of the monodisperse chain has been selected randomly from normal $\left(f^{(n)}(b)\right)$, uniform $\left(f^{(u)}(b)\right)$ and binary $\left(f^{(b i)}(b)\right)$ distributions whose standard deviations give the measure of the disorder of mass in the chain $(\xi)$. For instance, the normal distribution is given by

$$
f^{(n)}(b)=\frac{1}{\xi \sqrt{2 \pi}} e^{\frac{(b-1)^{2}}{2 \xi^{2}}} .
$$

High disorder means that the difference between the lightest particle and the heaviest particle is very large. It was observed in [98] that the three distributions showed quantitatively similar behavior if the first two moments of the distributions were the same. Here, the first two moments of the aforementioned three distributions have been matched. The mathematical details of the distributions are given in Appendix C. Ensembles of chains with different realizations for a particular disorder and distribution have been taken into consideration. Angular brackets will be used to denote ensemble averaged physical quantities like $\langle\mathbf{u}\rangle,\left\langle E_{\text {tot }}\right\rangle$, etc. The first five moments of the three distributions for different disorder (standard deviation) $\xi=0, \xi=0.1, \xi=0.2, \xi=0.35, \xi=0.5$ and $\xi=0.8$ are given in Table 2.1 (500 ensembles scaled), Table 2.2 (500 ensembles unscaled) and Table 2.3 (10000 ensembles). 


\subsubsection{Participation Ratio \& Localization Length}

The participation ratio $\left(P_{j}\right)$ (introduced in [22] and used previously in [7], [225]) is a crucial tool in determining the localization length $\left(\tilde{L}_{j}\right)$ associated with a particular eigenmode. This localization length can be seen as the length beyond which elastic waves with a particular frequency become evanescent, i.e., they decay exponentially in a disordered system ([128]). It is instrumental in determining the length within which the elastic waves become confined in space and is dependent on the frequency and thus the eigenmode ([9]). The participation ratio of eigenmode $j$ is defined as

$$
P_{j}=\frac{1}{\sum_{i=1}^{N}\left(S_{i j}\right)^{4}}
$$

with the normalization condition on the eigenmodes $\sum_{i=1}^{N}\left(S_{i j}\right)^{2}=1$. For one dimension, the localization length is defined as $\tilde{L}=P_{j} \tilde{d}$ where $\tilde{d}$ is the particle center distance in equilibrium, i.e. under pre-compression. The localization length can now be non-dimensionalised by the internal particle scale of separation $\sim \tilde{d}$ to give $L_{j} \cong P_{j}$. As discussed and pointed out in [7], the localization length of the lowest eigenmode is often attributed to the length of the chain (which would be regarded as a force chain in our analysis) and hence, it becomes important to find the localization length of an ordered chain, $\xi=0$ as reference. For an ordered chain $b^{(1)}, b^{(2)}, b^{(3)}, \ldots, b^{(N)}=1$ and $\kappa=1$, so,

$$
\mathbf{A}=\left[\begin{array}{cccccc}
-2 & 1 & 0 & 0 & \cdots & 0 \\
1 & -2 & 1 & 0 & \cdots & 0 \\
0 & 1 & \ddots & 0 & \cdots & 0 \\
0 & \cdots & 0 & \ddots & 0 & 1 \\
0 & \cdots & 0 & 0 & 1 & -2
\end{array}\right]
$$

The eigenvalues of this matrix are $\omega_{j}^{2}=2 \sin ^{2}\left(\frac{j \pi}{N}\right)$ and its eigenvectors are $\mathbf{s}_{(j)}=\left\{\sin \left(\frac{j \pi}{N}\right), \sin \left(\frac{2 j \pi}{N}\right), \sin \left(\frac{3 j \pi}{N}\right) \ldots \sin \left(\frac{(N-1) j \pi}{N}\right)\right\}$. After respecting the normalization condition and the definition of the participation factor, the localization length of the lowest eigenmode $\left(P_{0}\right)$ can be analytically calculated from the eigenvectors as

$$
P_{\text {norm }}=\sum_{i=1}^{i=N} \sin \left(\frac{i j \pi}{N}\right)^{2}, \text { and hence, } \frac{P_{0}}{P_{\text {norm }}^{2}}=\sum_{i=1}^{i=N}\left(\sin \left(\frac{i j \pi}{N}\right)\right)^{-4}
$$


For $N=256$, irrespective of $j=1,2,3 . . N, P_{0}=170.667 \approx 171$.

\subsubsection{Dispersion}

The analytical expression for the dispersion relation in an ordered chain of particles/elements with linear contact is given by ([28], [203], [97])

$$
\tilde{\omega}^{2}=4 \frac{\tilde{\kappa}_{o}}{\tilde{M}_{1}} \sin ^{2}\left(\frac{\tilde{k} \tilde{d}}{2}\right),
$$

where the wavenumber can be non-dimensionalized by the microscopic particle scale of separation $(\tilde{d})$ and frequency by $\sqrt{\frac{\tilde{\kappa}_{o}}{\tilde{M}_{1}}}$ giving the non-dimensional dispersion relation:

$$
\omega^{2}=\Omega_{\pi}^{2} \sin ^{2}\left(\frac{k}{2}\right)
$$

with $\Omega_{\pi}=2$ for ordered chains with $\xi=0$. Eq. (2.39) holds for propagative as well as evanescent waves. The positive roots of this relation correspond to propagative waves and the imaginary roots to evanescent waves ([203]). This expression also holds for longitudinal wave propagation in 3D granular packings ([129]) and in 1D chains as well ([97]). From the dispersion relation, it can be noted that disorder creates a maximum permissible frequency $\left(\Omega_{\pi}\right)$ for propagating waves, frequencies below $\Omega_{\pi}$ are propagative until the order of their localization length (Sect. 2.2.7) and the frequencies above $\Omega_{\pi}$ are evanescent. The dispersion relation (Eq. (2.39)) for ordered chains $(\xi=0)$ is

$$
\omega=2 \sin \left(\frac{k}{2}\right)
$$

which is the dispersion relation for propagative waves.

\subsubsection{Total Energy Dispersion}

From Eq. (A.6) it can be observed that the total energy of the eigenmodes is constant with respect to time as given by

$$
E_{\text {tot }}\left(\omega_{j}, k\right)=\frac{1}{2} \mathscr{A}_{j}(k)^{2} \omega_{j}^{2}
$$

By taking the first moment of this eigenmodal total energy representation about frequency, a dominant frequency related to a particular wavenumber can be 
obtained. Moments of the eigenmodal total energy representation are defined as

$$
M^{(m)}(k)=\frac{\sum_{j} \omega_{j}^{m} E_{\text {tot }}\left(\omega_{j}, k\right)}{\sum_{j} E_{\text {tot }}\left(\omega_{j}, k\right)} .
$$

The dominant frequency is given by the first moment,

$$
\Omega(k)=M^{(1)}(k)=\frac{\frac{1}{2} \sum_{j} \mathscr{A}_{j}^{2} \omega_{j}^{3}}{\sum_{j} E_{t o t}\left(\omega_{j}, k\right)} .
$$

The dominant frequency can be measured by averaging over all eigenmodes for a single realization with $\mathscr{A}_{j}(k)$ as a multiplicative factor which depends on the Fourier initial condition $\mathcal{V}_{o}(k)$ (Eq. (2.32)). The dispersion relation for the propagating waves can be obtained by taking ensemble averages of this dominant frequency $(\langle\Omega(k)\rangle)$, which will be plotted in Fig. 2.10(b) below for different disorder strengths (500 ensembles).

\subsubsection{Group velocity}

The group velocity is given by

$$
v_{g}=\frac{\partial \omega}{\partial k}
$$

for both propagative waves and evanescent waves. It can be obtained by differentiating Eq. (2.40) that

$$
v_{g}(k)=\frac{\sqrt{\Omega_{\pi}^{2}-\omega^{2}}}{2} .
$$

where $\Omega_{\pi}=\Omega_{\pi}(\xi)$ depends on disorder as we will see below.

\subsection{Results \& Discussions}

The analytical expressions derived in the previous sections are computed for $N=256$ particles long chains. The impulse imparted to the first particle is $v_{o}=$ 
0.05. The time step utilized for the output is, $\Delta t=0.0312$ and the maximum time up to which the computations have been carried out is $t_{\max }=256$ such that the pulse has just about reached the $256^{\text {th }}$ particle. As it can be seen from Tables 2.1 and 2.3, the scaled average mass of the particles has been kept $M_{1}=1$ and $\xi=0.0,0.1,0.2,0.35,0.5$ and 0.8 disorder parameters (standard deviation; see appendix) have been used for analysis. Using the analytical solution of the linearized system (Eq. (2.31)), ensembles of 500 and 10000 chains along with representative single realizations will be shown in this section.

\subsubsection{Nonlinear (Hertz) and Linear Space Time Responses}
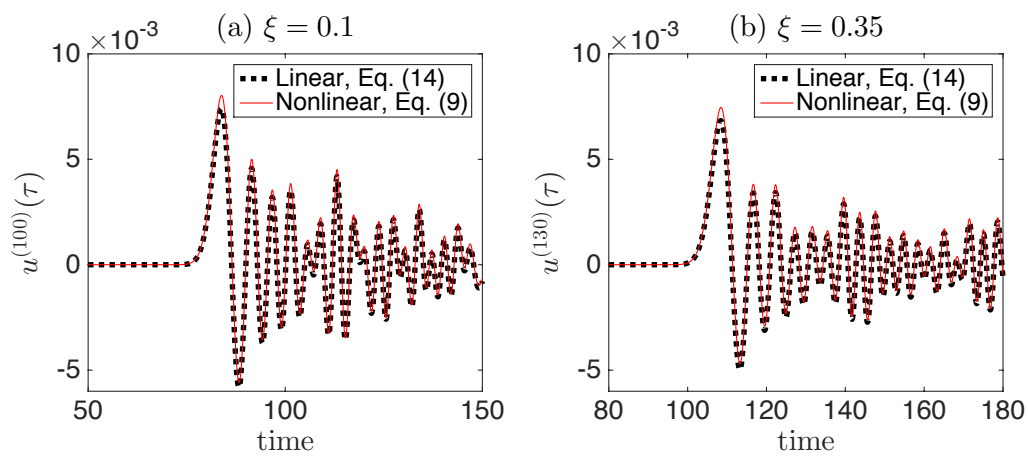

Figure 2.3: The displacement as a function of time is shown for $100^{\text {th }}$ particle in a chain of particles with disorder parameter, $\xi=0.1$ in Fig. 2.3 (a) and $130^{\text {th }}$ particle in a chain of particles with disorder parameter, $\xi=0.35$ in Fig. 2.3(b).

Equation (3.5) with Eq. (2.12) and Eq. (2.13) has been solved numerically with Verlet integration to get space time responses of particles having nonlinear (Hertzian) repulsive interaction force. The time step used for the numerical integration is $\Delta t=0.00038147$. Fig. 2.3 shows the space time responses calculated numerically for the nonlinear equations of motion (Eq. (3.5)) and the space time responses calculated for the linearized equation of motion (Eq. (2.14)). The space time responses are obtained for a single realization of a granular chain, no ensemble averaging has been done here. The nonlinear space time responses coincide with the linear space time responses, confirming that the solution given by Eq. (2.31) is also appropriate for particles with nonlinear repulsive interaction forces for small displacements. In order to observe 

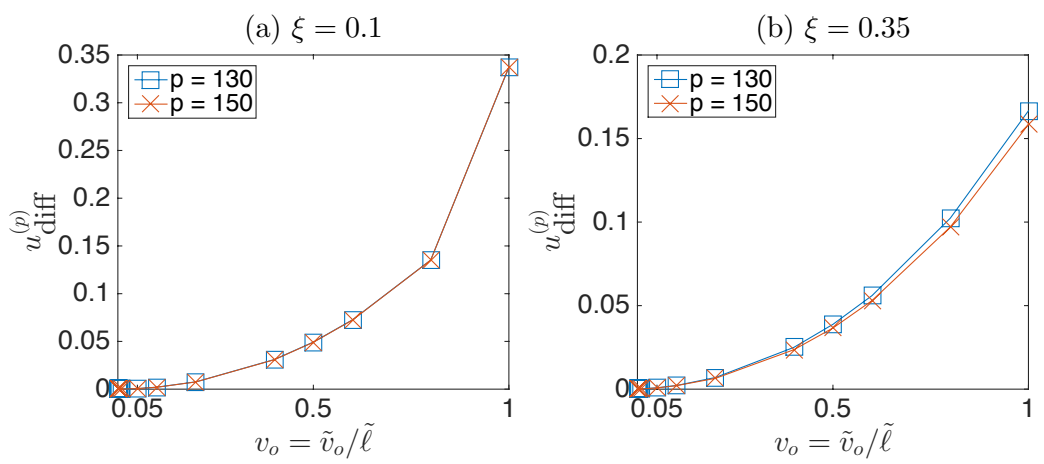

Figure 2.4: The nonlinear increase in $u_{\text {diff }}^{(p)}$ (a parameter which shows dissimilarity between linear and nonlinear space time responses) with initial impulse velocity $\left(v_{o}\right)$. The value $v_{o}=0.05$ is in the zone where linear and nonlinear space time responses are the same.

the limitation of the linear space time responses obtained from Eq. (2.31), Fig. (2.4) is plotted. The difference between the maximum value $\left(u_{\text {peak }}\right)$ of the space time responses for hertzian and linear repulsive interaction force $\left(u_{\text {diff }}^{(p)}=u_{\text {peak(hertz) }}^{(p)}-u_{\text {peak(linear) }}^{(p)}\right)$ is chosen as a parameter to judge the appropriateness of linear space time response for the nonlinear equation of motion (Eq. (3.5)). Figures show that the difference between nonlinear (Hertz) and linear space time response increases nonlinearly irrespective of particle position and disorder parameter of the granular chain.

\subsubsection{Displacement Response of the Three Distributions}

The mass disorder of the particles in the chain is taken into consideration only and $\kappa$ is chosen as 1 (Sect. 2.2.3). Figure 2.5 shows the displacement as a function of time of the $150^{\text {th }}$ particle (Fig. 1(a) \& 1(c)) and of the $220^{\text {th }}$ particle (Fig. 1(b) \& 1(d)), which are placed before and after the reference localization length (the maximum possible, $L_{\max }=171$, Sect. 2.3.7) for two disorder parameters, $\xi=0.1$ and $\xi=0.5$ with three mass distributions (normal, uniform and binary). For weak disorder $(\xi=0.1)$, it is observed that the displacement wave packets are perfectly superposed over each other affirming what was concluded in [98] \& [97] that the shape of the distribution has no effect on the propagating pulse if the first two moments of the distribution are the same (Ta- 
(a)

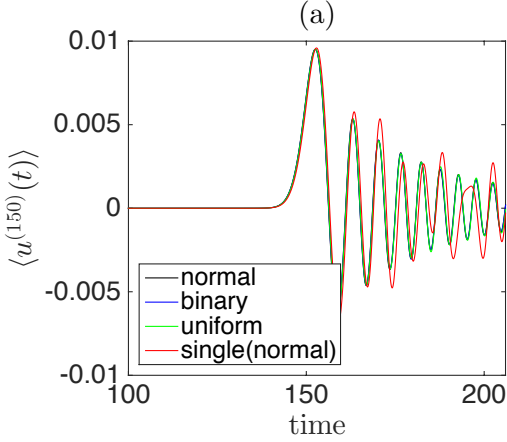

(c)

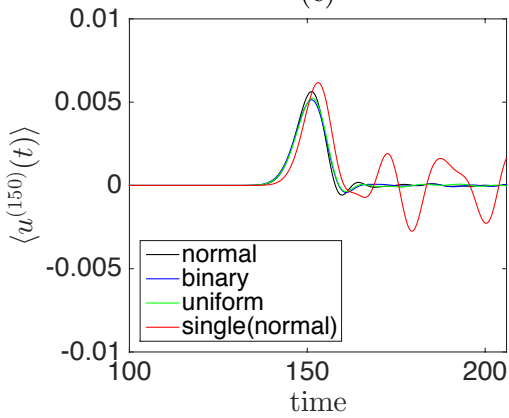

(b)

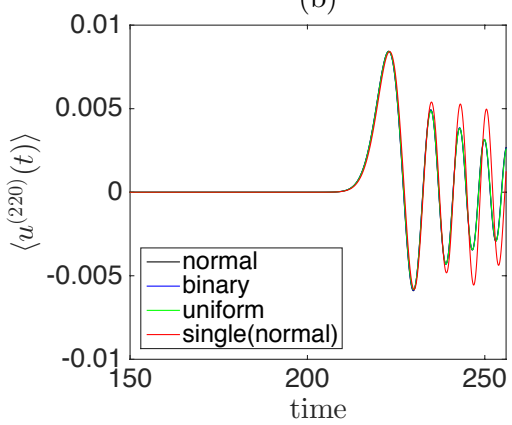

(d)

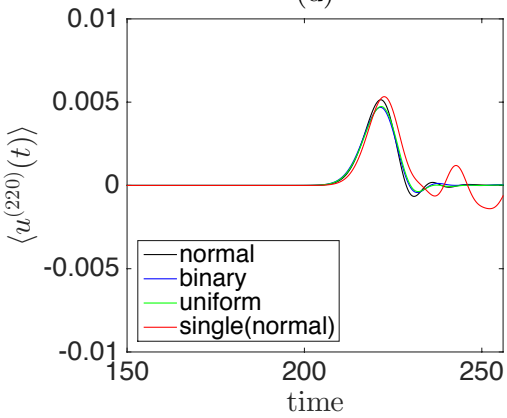

Figure 2.5: Ensemble averaged displacements (500 times) of $150^{\text {th }}$ (Fig. 2.5(a) and Fig. 2.5(c)) and $220^{\text {th }}$ (Figures 2.5(b) and 2.5(d)) particle with respect to time. Figures 2.5(a) and 2.5(b) have disorder parameter, $\xi=0.1$, Fig. 2.5(c) and Fig. 2.5(d) have disorder parameter, $\xi=0.5$. The red plot is the space time response from a single realization of a chain with normally distributed masses. $\forall$ single realization, normal distribution is used with $\tilde{M}_{1}=0.9971$ and $\tilde{M}_{2}=1.1274$ for $\xi=0.1, \tilde{M}_{1}=0.9971$ and $\tilde{M}_{2}=1.1274$ for $\xi=0.5$ and $\tilde{M}_{1}=0.9958$ and $\tilde{M}_{2}=1.2636$ for $\xi=0.5$ 
ble 2.1). For stronger disorder $(\xi=0.5)$, the wave packets are not collapsing perfectly (Fig. 1(c) \& 1(d)). As it can be seen in Table 2.1 that there is a numerical mismatch between the unscaled moments of the distributions leading to a dissimilarity between the second scaled moments $\left(\left\langle M_{2}\right\rangle\right)$. This also causes the real standard deviation (disorder; $\xi$ ) which has been numerically calculated ( $\Xi$; Table 2.1) to deviate a little bit from it's intended value. It can also be observed from Fig. 2.5 (c) and (d)) that the pulse shapes of binary distribution and uniform distribution are closer to each other in comparison to normal and binary or normal and uniform, since, the scaled second moments $\left(\left\langle M_{2}\right\rangle\right)$ of binary and uniform distributions for $\xi=0.5$ are closest to each other (Table 2.1). Similar conclusions about similarity, dissimilarity and closeness can be drawn about pulse shapes of different distributions for different disorder parameters ( $\xi$ (intended), $\Xi$ (numerically obtained)) on the basis of moments of the mass distribution. For larger $\xi$, higher moments have to be considered ([144]), but discussing these higher moments and their consequences go beyond the scope of this study.

\subsubsection{Displacement Response for Different Disorder}

Mechanical waves propagating through disordered media or granular media like soil (on the receiver end) can be divided into two parts, the coherent part and the incoherent part ([77], [76]). The coherent part is the leading wave packet and self averaging in nature (it maintains its shape after ensemble averaging), it is used for determining bulk sound wave velocity. In contrast, the incoherent part is the scattering, non-self averaging part, which is strongly system configuration dependent, also known as coda or tail of the mechanical wave. Figure 2.6 contains displacement of the same particles ( $150^{\text {th }} \& 220^{\text {th }}$ particles) used in subsection 2.3.2 for consistency. Here, attention has been given to the effect of the mass distribution on the time of arrival/flight and hence, the wave velocity of the initial wave packet. Figures 2.6(a) and 2.6(b) contain the displacements of the $150^{t h}$ and $220^{t h}$ particle (before and after the $L_{\text {max }}$, Sect. 2.3.7) for single realization, 500 ensembles and 10000 ensembles. The leading wave packet is the same for 500 ensembles and 10000 ensembles in both the figures, i.e. the coherent part of the wave which maintains its shape after averaging. The coda is more or less pronounced at 150 or 220 respectively and vanishes due to ensemble averaging. Figures 2.6(c) and 2.6(d) show the displacement response of $150^{\text {th }}$ and $220^{\text {th }}$ particles with respect to time for chains with different mass disorder. The speed of the coherent wave packet (from source to receiving particle) is increasing with disorder. Higher disorder leads 
(a)

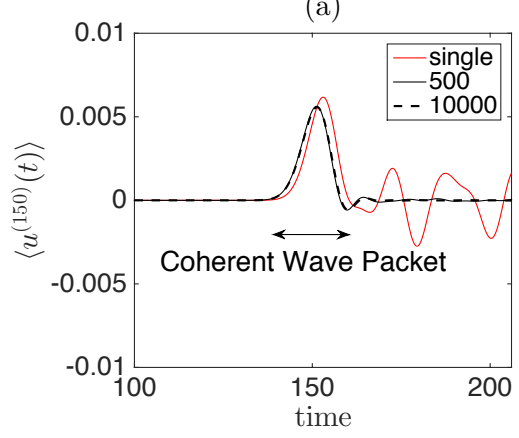

(c)

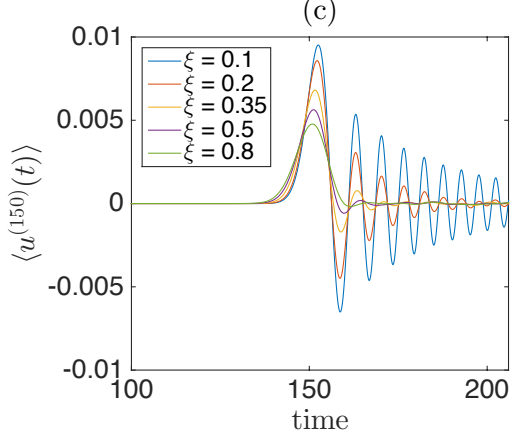

(b)

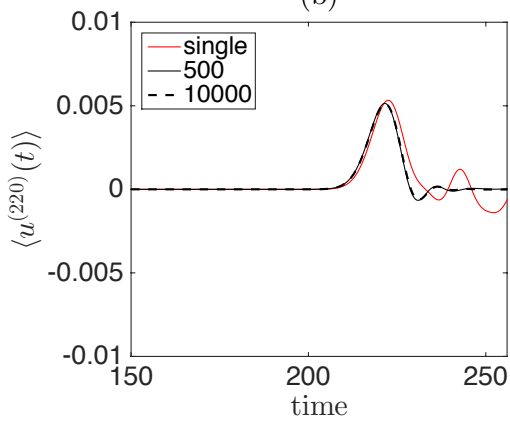

(d)

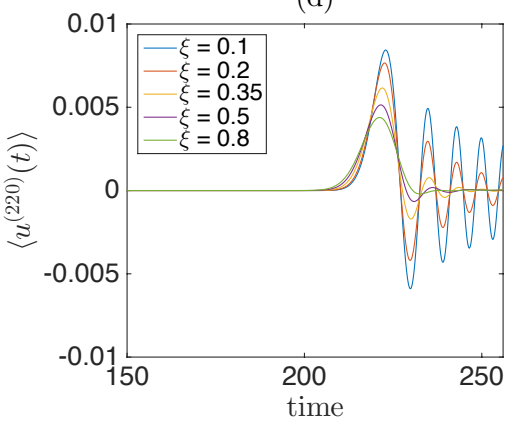

Figure 2.6: Displacements of $150^{\text {th }}$ (Fig. 2.5(a) and Fig. 2.5(c)) and $220^{\text {th }}$ (Fig. 2.5(b) and Fig. 2.5(d)) particle with respect to time for different ensembles (Fig. 2.5(a) and Fig. 2.5(b)) of disorder, $\xi=0.5$ and of multiple disorder (Fig. 2.5(c) and Fig. 2.6(d); for 500 ensembles). 
to higher coherent wave speed, irrespective of the localization length $\left(L_{\max }\right)$. However, this increase in wave speed can also be attributed to sound wave accelaration near the source as pointed out by [130] and may not be generalized as effect of mass disorder in the chain, this has been investigated in the next section.

\subsubsection{Coherent Wave Speed and Disorder}

Table 2.4 contains the velocity of the peak of the coherent wave, the velocity of the rising part of the coherent wave packet when the displacement of the particle has attained $5 \%, 10 \%, 70 \%, 90 \%$ of the peak value and the first time when the displacement of the particle becomes 0 after it has attained the peak value of the coherent wave (zero crossing), all constituting the coherent wave packet. The results have also been plotted in Fig. 2.7. The velocities were determined through velocity picking (particle position divided by the time of arrival). The particles used for computing the velocities were 130, 150, 200 and 220 ( 2 before localization length and 2 after localization length, $L=171$ ). It can be observed that irrespective of the rising part of the coherent wave packet (5\%, $10 \%$, of the peak value etc.) and the peak (Fig. 2.7(a), (b), (c), (d)), the wave velocity increases with disorder. However, for zero crossing (Fig. 2.7(e)), the velocity decreases with increase in disorder and the same can be said for the part of the coherent wave packet which lies after the peak value, this can be attributed to the increased spreading of the wave packet with increase in disorder. Fig. 2.7(f) shows the velocity of the peak value of the coherent wave packet of all the particles of the granular chain for different disorder parameters and it also exhibits a similar kind of acceleration of signal/mechanical wave near the source as was observed in [130]. This acceleration is caused by selfdemodulation of the initial impulse imparted to the granular chain and the noteworthy point is that the acceleration increases with increase in disorder. However, due to this observation we cannot generalize the effect of disorder on wave speed. The sudden rise in velocity of the peak value in Fig. 2.7(f) after the $250^{\text {th }}$ particle is due to the boundary effect as well as due to the presence of coherent wave front of the traveling wave around that position as the maximum time window used is $t_{\max }=256$.

To understand the effect of disorder on wave speed without taking into account this "source effect", the time taken by the pulse for propagating a common distance of separation has been computed and the velocity of the wave computed by using it (common distance of separation divided by the time taken by the pulse to travel) has been taken in Table 2.6 ( 7 particles as the common 
(a)

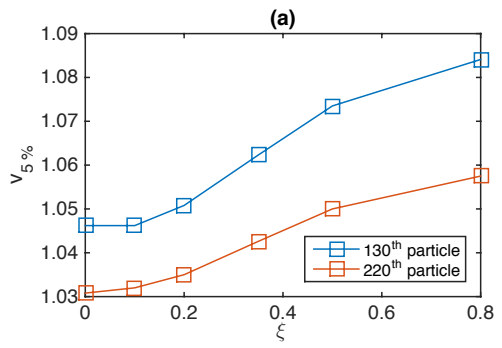

(c)

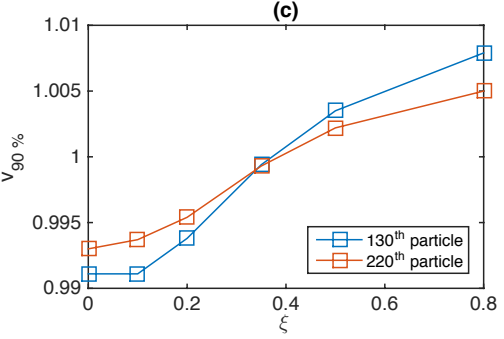

(e)

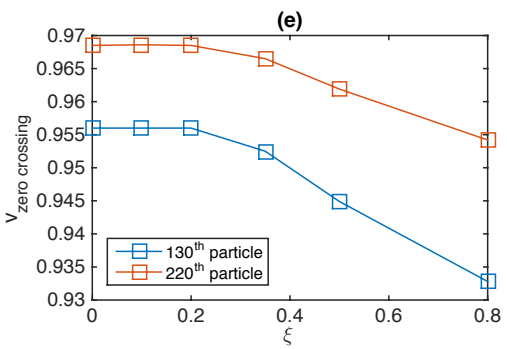

(b)

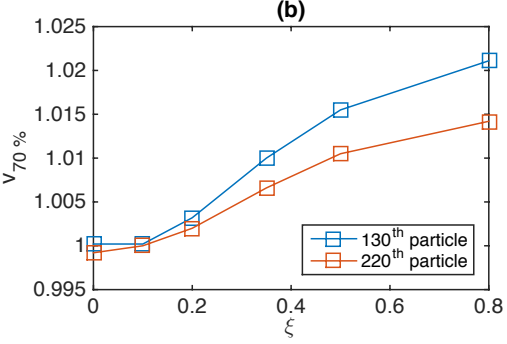

(d)

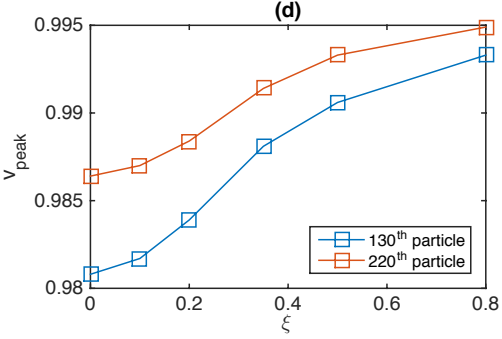

(f)

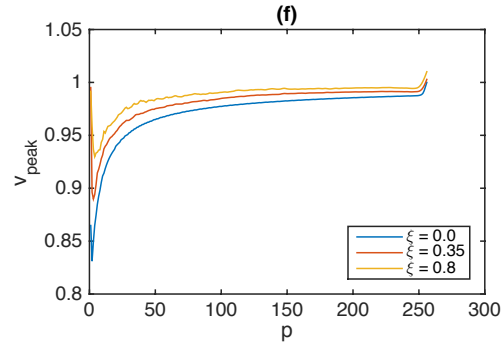

Figure 2.7: Coherent Wave Velocities determined through Velocity picking. The peak of the coherent wave packet's velocity (Fig. 2.7(d)) and the rising part of the packet (Fig. 2.7(a), (b) and (c)) as well as the falling part (Fig.2.7(e)) are taken into consideration. Fig.2.7(f) plots the peak velocity for all the particles in a granular chainwith different $\xi$ 
distance of separation), the results have also been plotted in Fig. 2.8. The reason for selecting such a low common distance of separation was to keep the effect of the source as minimal as possible. The velocities have been computed from the time taken by the pulse to travel between different sets of points (130 to 137,150 to 157,220 to 227 and 240 to 247 particles) with use of different reference points of the coherent wave front $(5 \%, 10 \%, 70 \%, 90 \%$, peak value and zero crossing). From Fig. 2.8 and Table 2.6 it can be observed that the velocities computed using different reference points follow the same trend except the velocity computed using zero crossing reference point. Velocities computed using zero crossing reference point are more or less constant with little fluctuations (Table 2.6). Fig. 2.8(a) shows a consistent increase of velocity as it is the closest to the source (dominated by the source effect), however, as the set of particles is selected farther away from the source, the velocity trend shows a slight decrease and then an increase with the increase in $\xi$ (Fig. 2.8(b) and (c)). Fig. 2.8(d) exhibits a consistent decrease of velocity with increase in $\xi$ because the set of particles (247-240) are far from the source (source effect is weak). From Fig. 2.8(d), it can be interpreted that higher disorder results in decrease in wave velocity.

\subsubsection{Frequency Response \& Dispersion}

In Fig. 2.9(a) \& Fig. 2.9(c) a Fast Fourier Transform (FFT) with respect to time is carried out on the displacement response of a 256 element long chain for disorder, $\xi=0.01$ and $\xi=0.35$ respectively (when an impulse of $v_{o}=0.05$ has been applied to the first particle) to observe the frequency content with distance, (the sampling frequency is $\omega_{\text {sample }}=\frac{2 \pi}{\Delta t}$ ) and responses upto half of the sampling frequency were taken into account to avoid aliasing. The first 5 particles have been excluded from the Fourier transform to avoid the driving effect.

Fig. 2.9(a) exhibits the existence of cut-off frequency $(\omega=2)$ above which the waves become evanescent. The bending of the density with distance (particle number) especially at large distances is attributed to dispersion and the finite time window which is well encapsulated (red curve) by the analytical solution given in Eq. (2.47) using the group velocity ( $p$ is the particle number)

$$
v_{g} t_{\max }=p,
$$

For an ordered chain $(\xi=0), \Omega_{\pi}=2$ and on using Eq (2.45), the previously 
(a)

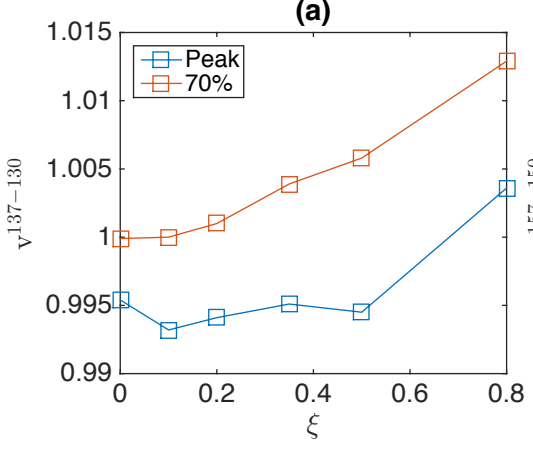

(c)

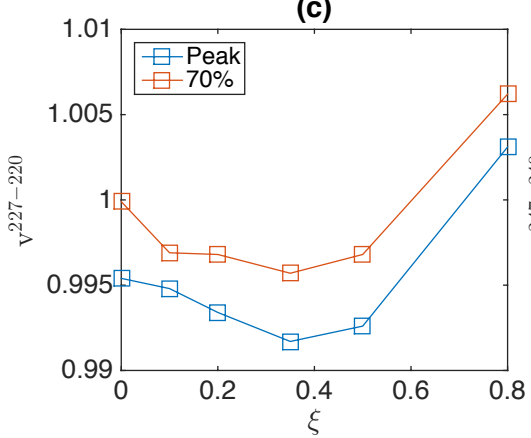

(b)

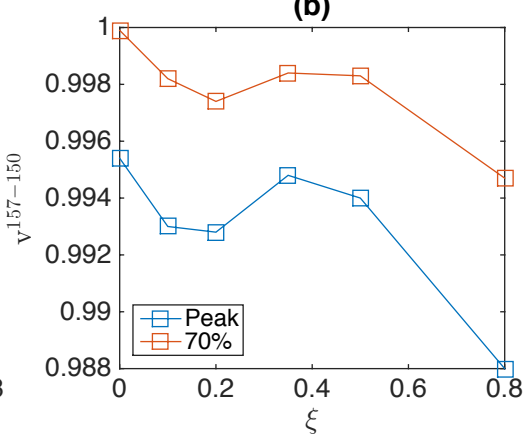

(d)

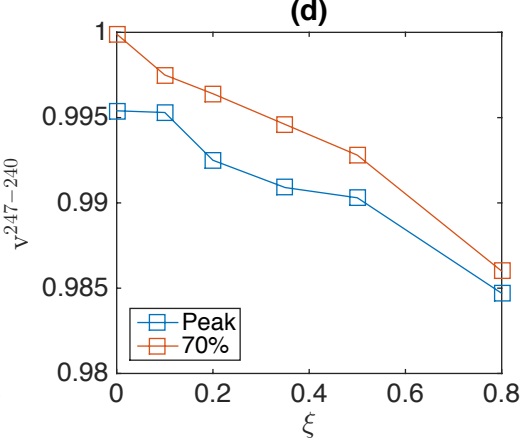

Figure 2.8: Wave speed for common distance of separation (7 particles/elements) with different disorder $\xi$.

mentioned equation can be written as

$$
\omega(p)=2 \sqrt{1-\frac{p^{2}}{t_{\text {max }}^{2}}},
$$

which is the red curve plotted in Fig. 2.9(a).

A spatial as well as temporal 2D FFT is carried out for a single realization of a 256 element long chain with disorder $\xi=0$ and $\xi=0.35$ to observe the dispersion relation (Fig. 2.9(b) \& 2.9(d); $\omega \mathrm{v} / \mathrm{s} k$ ). 2D FFT has been used previously for one-dimensional and three dimensional polydisperse granular packings for 
(a) $\xi=0.01,1 \mathrm{D} \mathrm{FFT}$

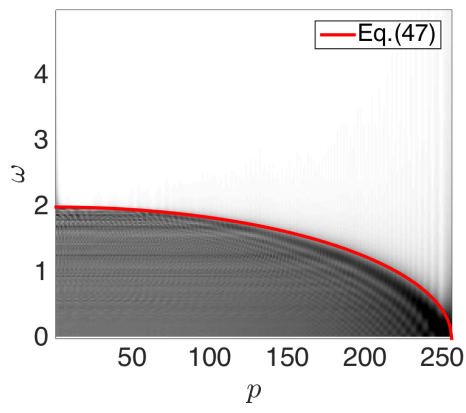

(c) $\xi=0.35,1 \mathrm{D} \mathrm{FFT}$

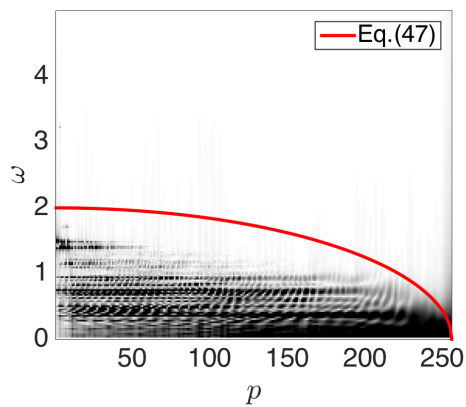

(b) $\xi=0.01,2 \mathrm{D} \mathrm{FFT}$

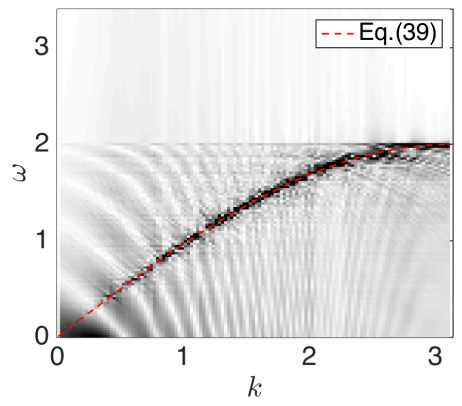

(d) $\xi=0.35,2 \mathrm{D} \mathrm{FFT}$

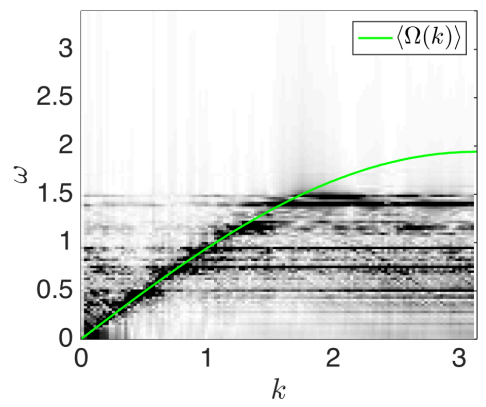

Figure 2.9: Figure 2.9(a) is the temporal Fourier transform of displacement of particles for disorder parameter $\xi=0.01$ (single realization) with group velocity $\left(v_{g}\right)$ depicting the propagation of wave front and Fig. 2.9(b) is the temporal as well as spatial Fourier transform (2 D FFT, single realization) calculated for obtaining dispersion relation of a chain, $\langle\Omega(k)\rangle$ gives the true dispersion relation. Fig. 2.9(c) and (d) are $\xi=0.35$ counterparts of Fig. 2.9(a) and (b) respectively. 
obtaining dispersion relations ([97], [143], [106]) but strong frequency filtering due to the disordered system resulted in ambiguous dispersion relations (flat bands and absence of certain frequencies below the cut-off frequency which is the propagative band due to the presence of defect modes). This can also be observed from the density plots in the Fig. 2.9(b) and Fig. 2.9(d). Eq. (2.40) (the dispersion relation for an ordered chain) has been plotted in Fig. 2.9(b) which gives a perfect fit for the denser regime in the figure. However for the disordered chain, $\xi=0.35$, as proposed earlier in Sect. 2.2.9, the dispersion relation can be given by $\langle\Omega(k)\rangle$ by ensemble averaging the dominant frequencies with respect to different wavenumbers. $\langle\Omega(k)\rangle$ for 500 ensembles with disorder $\xi=0.35$ has been plotted in Fig. 2.9(b) (the green curve). It can be seen that for low frequencies the green curve perfectly superposes the dense regime in the density plot of displacement's temporal and spatial Fourier transform, for higher frequency due to the appearance of the flat band (defect mode) the density of frequencies are not present near the green curve. But, it can be noted that the green curve holds true for low and meso-level frequencies/wavenumbers.

\subsubsection{Total Energy Dispersion in Disordered Chains}

The $\langle\Omega(k)\rangle$, which was plotted for $\xi=0.35$ in Fig. 2.9(b) is plotted for $\xi=$ $0.1,0.2,0.35,0.5$ and 0.8 in Fig. 2.10(a). It is observed that maximum permissible frequency $\left(\Omega_{\pi}\right)$ above which the waves become evanescent decreases with increasing disorder. The slope of $\omega \mathrm{v} / \mathrm{s} k$ curves indicates the wave speed which clearly can be observed to be decreasing with increasing disorder. This indicates that the wave speed is decreasing with increasing disorder, confirming what was observed in Sect. 2.3.4.

\subsubsection{Participation Ratio \& Localization length}

Figure 2.11 shows the participation ratio $(\langle P\rangle)$, i.e. the localization length $(\langle L\rangle$, from Sect. 2.2.7) for binned 500 ensemble averaged realizations of chains (with 0.0781 as frequency bin size) and with different disorder parameters, $\xi=0.1,0.2,0.35,0.5$ and 0.8 . The lowest frequencies always have the same localization length which is $L_{\max }=171$, independent of the disorder of mass in the chain. In Sect. 2.2.7, $L_{\max }$ has been derived analytically from an ordered chain and through the definition of the participation ratio. Towards higher frequency it can be observed that the localization length becomes same for all the disordered chains at a particular frequency (cross-over frequency). For frequencies 
(a)

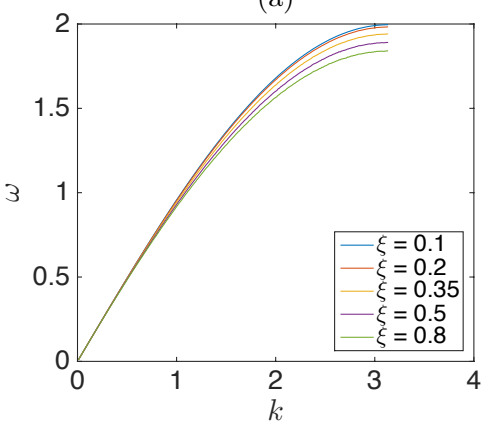

(b)

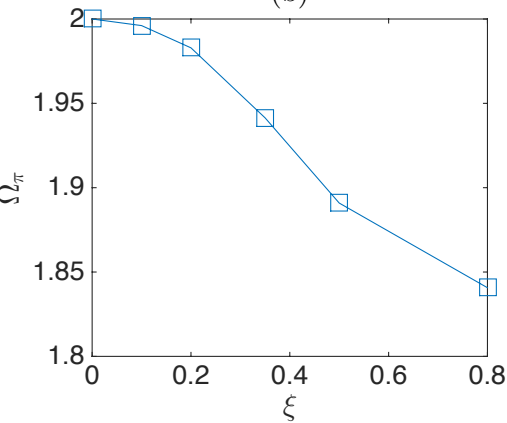

Figure 2.10: Dispersion relation (Fig. 2.10(a), $\langle\Omega(k)\rangle$ ) with respect to different wavenumbers and the maximum permissible frequency has been shown for disorder parameters $\xi=0.1,0.2,0.35,0.5$ and 0.8

above the cross-over frequency, the localization length increases with increasing disorder and for frequencies below the cross-over frequency, the localization length decreases with increasing disorder. Unlike infinitely long chains where $L \propto \omega^{-2}$ (as suggested in [13]), the finite disordered chains for higher frequencies have $L \propto \omega^{-q}$ where $q>2$ and decreases with increasing disorder.

(a)

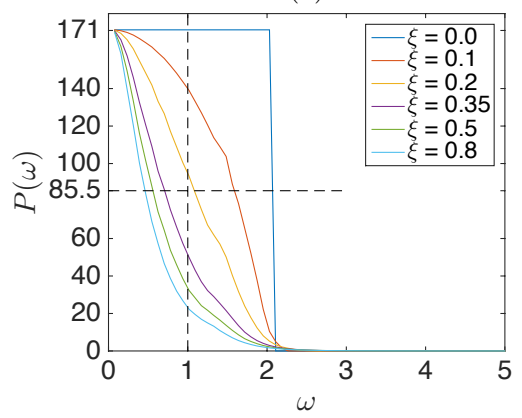

(b)

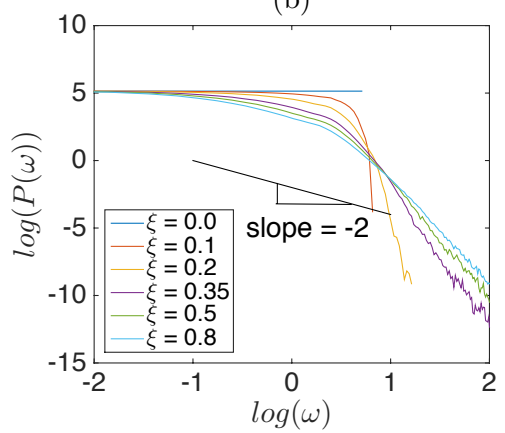

Figure 2.11: Participation Ratio or the Localization length with respect to different frequencies for 500 ensembles with $\xi=0.1,0.2,0.35,0.5$ and 0.8 and bin size $=$ 0.0781 .

For understanding the effect of disorder on cut-off frequency $\left(\omega_{\text {pass }}\right)$ associ- 
ated with the localization lengths and the localization length $(L), \omega_{\text {pass (1/2) }}$ (the cut-off frequency associated with half the value of $L_{\max }$ ) for different disorder parameters have been plotted in Fig. 2.12(a) and $L$ associated with $\omega=1$ scaled with $L_{\max }$ for multiple disorder parameters have been plotted in Fig. 2.12(b). The values have been selected from the dashed lines in Fig. 2.11(a). Both of the quantities exhibit a decreasing trend with increase in disorder in the pass band of frequencies $\left(0-\Omega_{\pi}\right)$.

(a)

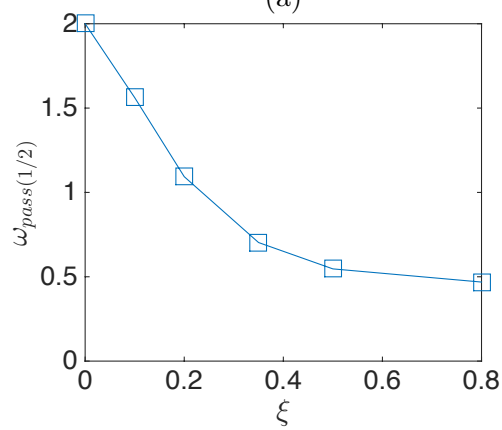

(b)

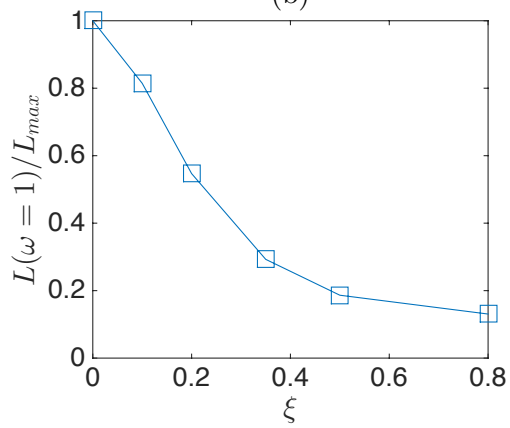

Figure 2.12: (a) $\omega_{\text {pass (1/2) }}$ for different $\xi$. (b) Localization length scaled with maximum localization length $\left(L_{\max }\right)$ for different $\xi$.

\subsubsection{Total Energy of Eigenmodes}

The Density Of States (DOS) or density of vibrational modes is treated as an important quantifying factor in studying the vibrational properties of materials like crystals, jammed granular media ([169]), etc. However, it tells us only about the number of vibrational modes but, does not paint the complete picture of spectral properties of vibration like energy transport. Eq. (A.5) gives us the total energy of individual eigenmodes and also from the equation, we can deduce that the energy is constant with respect to time. Figure 2.13(a) plots the ensemble averaged density of states for 500 mass disordered granular chains with frequency bins of size 0.0781 . The peak of the density is decreasing with increasing disorder and shifting to smaller $\omega$. Figure 2.13(b) gives the ensemble averaged constant energy spectrum for the same frequency bins used in the previous plot (500 realizations) giving an energy distribution over frequency. The shape of the energy distribution is wider over frequency for low $\xi$ but, for 

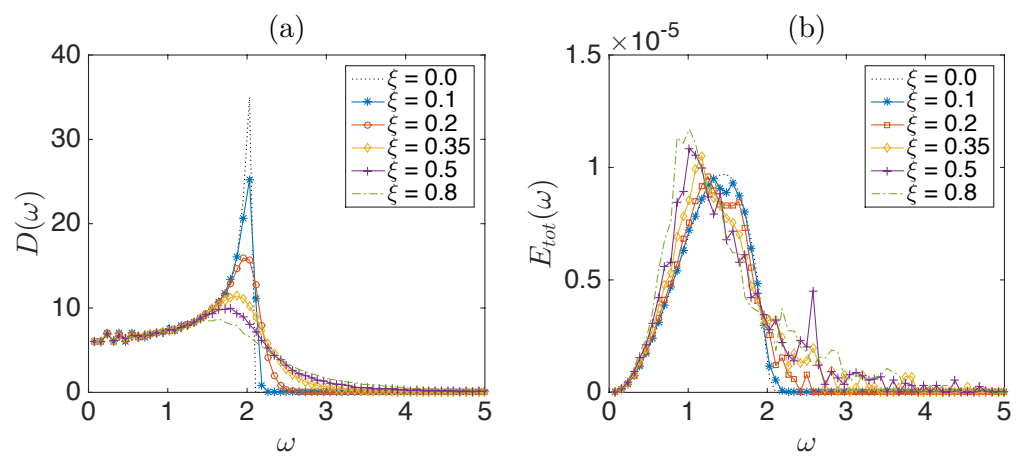

Figure 2.13: (a) Density of States (DOS) and (b) energies of the binned frequencies for different disorder $\xi$.

higher $\xi$, the energy distribution becomes more sharp with a peak. The peak increases with increasing disorder. In both plots Fig. 2.13 (a) and (b), the tails are broader for larger $\xi$.

\subsection{Conclusions}

An impulse driven wave propagating through a precompressed mass disordered granular chain has been studied. Motivation comes from the existence of force chains which form the backbone network for mechanical wave propagation in granular materials like soil. The scaled standard deviation of the mass probability distribution of the elements/particles of the granular chain has been identified as the relevant disorder parameter ( $\xi$; see Sect. 2.2.6), as suggested already in [97]. Chains with normal, binary and uniform mass distributions have quantitatively identical signal transmission characteristcs as long as the first two moments of the mass distribution are the same and $\xi$ is not too large.

Interestingly, on first sight, the dependence of wave speed on magnitude of disorder looks non-monotonous. This surprising increase of wave-speed for weak disorder, and decrease for stronger disorder, is due to two different effects overlapping: The increase of wave-speed takes place close to the source, see Fig. 2.7, i.e. our 1D granular chain has the ability to model the physics of accelerating waves, as observed in complex higher dimensional granular structures ([130]). The competing mechanism of decreasing wave-speed with disorder is only clearly observed when the velocities are measured as travel time with main- 
taining constant separation far away from the source (Fig 2.8 and Table 2.6). The group velocity given by Eq. (2.45) also shows a decrease in wave-speed with increase in disorder. When the travel time is measured from the source, the two mechanisms overlap and interfere, causing the non-monotonous behavior, but possibly allowing for tuning particular propagation characteristics in short chains.

As another main result, Eq. (2.43) gives an effective, weighted dispersion relation as the normalized first moment of eigenmodal (total) energies with frequency. This gives a much better signal to noise ratio for $\omega \mathrm{v} / \mathrm{s} k$ in comparison to 2D FFT of displacement or velocity signals, reported previously ([130], [143]). Figure 2.10 shows that the upper limit (maximum permissible) frequency due to the discreteness of the system slightly decreases with increasing disorder, $\xi$, and consistently, waves propagate slightly slower with increasing disorder. From the energy content one also observes (in disordered systems) that waves above a low frequency pass-band $\left(\omega_{\text {pass }}\right)$ become evanescent after they have traversed a localization length, $L=L(\omega)$, associated with this particular cut-off frequency $\left(\omega_{\text {pass }}\right)$.

The energy analysis presented in this article can be used for understanding pulse propagation in disordered, weakly or strongly non-linear granular chains and its attenuation, widening and acceleration (experimentally and numerically investigated in [93]). It would be interesting to understand the effect of damping on the eigenmodes, velocity of the propagating wave, change in frequency filtering and the energy of the eigenmodes. Also, a different kind of averaging (micro-macro transition) should be developed using frequency bands to develop a Master Equation for propagation (or localization) of total energy in terms of wavenumber and frequency at different regimes of disorder, non-linearity, and material properties. Such macro-models, taking into account multiple scattering, dispersion, attenuation, etc. will allow for modeling of realistic wave propagation in granular materials like soil on large scales. 
Table 2.1: Scaled Moments of ensemble averaged distributions (500 ensembles) used for the one-dimensional chain (256 element long).

\begin{tabular}{|lcccccccc|}
\hline \multicolumn{1}{|c}{ Distribution } & Disorder & $\left\langle M_{1}\right\rangle$ & $\left\langle M_{2}\right\rangle$ & $\left\langle M_{3}\right\rangle$ & $\left\langle M_{4}\right\rangle$ & $\left.<M_{5}\right\rangle$ & $\Xi$ & $\Xi^{2}$ \\
& $\xi=0.0$ & 1.0000 & 1.0000 & 1.0000 & 1.0000 & 1.0000 & 0.0000 & 0.0000 \\
& $\xi=0.1$ & 1.0000 & 1.0099 & 1.0298 & 1.0600 & 1.1010 & 0.1 & 0.0100 \\
Normal & $\xi=0.2$ & 1.0000 & 1.0398 & 1.1194 & 1.2436 & 1.4219 & 0.1999 & 0.0400 \\
Distribution & $\xi=0.35$ & 1.0000 & 1.1190 & 1.3590 & 1.7630 & 2.4184 & 0.3462 & 0.1195 \\
& $\xi=0.5$ & 1.0000 & 1.2053 & 1.6366 & 2.4335 & 3.8973 & 0.4661 & 0.2061 \\
& $\xi=0.8$ & 1.0000 & 1.3055 & 2.0104 & 3.5037 & 6.7333 & 0.6415 & 0.3067 \\
\hline \multirow{3}{*}{ Binary } & $\xi=0.0$ & 1.0000 & 1.0000 & 1.0000 & 1.0000 & 1.0000 & 0.0000 & 0.0000 \\
Distribution & $\xi=0.1$ & 1.0000 & 1.0100 & 1.0299 & 1.0599 & 1.1001 & 0.1000 & 0.0100 \\
& $\xi=0.2$ & 1.0000 & 1.0398 & 1.1196 & 1.2408 & 1.4068 & 0.2000 & 0.0400 \\
& $\xi=0.35$ & 1.0000 & 1.1221 & 1.3666 & 1.7489 & 2.2998 & 0.3501 & 0.1226 \\
& $\xi=0.5$ & 1.0000 & 1.2495 & 1.7497 & 2.5653 & 3.8255 & 0.5002 & 0.2505 \\
& $\xi=0.8$ & 1.0000 & 1.6413 & 2.9323 & 5.3034 & 9.6263 & 0.8014 & 0.6438 \\
\hline Uniform & $\xi=0.0$ & 1.0000 & 1.0000 & 1.0000 & 1.0000 & 1.0000 & 0.0000 & 0.0000 \\
Distribution & $\xi=0.1$ & 1.0000 & 1.0100 & 1.0300 & 1.0602 & 1.1009 & 0.1002 & 0.0100 \\
& $\xi=0.2$ & 1.0000 & 1.0400 & 1.1201 & 1.2431 & 1.4148 & 0.2004 & 0.0402 \\
& $\xi=0.35$ & 1.0000 & 1.1227 & 1.3682 & 1.7639 & 2.3646 & 0.3508 & 0.1232 \\
& $\xi=0.5$ & 1.0000 & 1.2508 & 1.7529 & 2.6212 & 4.0859 & 0.5011 & 0.2517 \\
& $\xi=0.8$ & --- & --- & --- & --- & --- & --- & --- \\
\hline
\end{tabular}


Table 2.2: Unscaled Moments of ensemble averaged distributions (500 ensembles) used for the one-dimensional chain (256 element long).

\begin{tabular}{|lcccccccc|}
\hline \multicolumn{1}{|c}{ Distribution } & Disorder & $<\tilde{M}_{1}>$ & $<\tilde{M}_{2}>$ & $<\tilde{M}_{3}>$ & $<\tilde{M}_{4}>$ & $<\tilde{M}_{5}>$ & $\tilde{\Xi}$ & $\tilde{\Xi}^{2}$ \\
& $\xi=0.0$ & 1.0000 & 1.0000 & 1.0000 & 1.0000 & 1.0000 & 0.0000 & 0.0000 \\
& $\xi=0.1$ & 1.0000 & 1.0100 & 1.0299 & 1.0601 & 1.1013 & 0.0999 & 0.0100 \\
Normal & $\xi=0.2$ & 1.0000 & 1.0399 & 1.1197 & 1.2443 & 1.4232 & 0.1999 & 0.0400 \\
Distribution & $\xi=0.35$ & 1.0022 & 1.1242 & 1.3689 & 1.7807 & 2.4492 & 0.3462 & 0.1195 \\
& $\xi=0.5$ & 1.0274 & 1.2728 & 1.7768 & 2.7163 & 4.4725 & 0.4661 & 0.2061 \\
& $\xi=0.8$ & 1.1581 & 1.7540 & 3.1363 & 6.3458 & 14.1470 & 0.6415 & 0.3067 \\
\hline \multirow{4}{*}{ Binary } & $\xi=0.0$ & 1.0000 & 1.0000 & 1.0000 & 1.0000 & 1.0000 & 0.0000 & 0.0000 \\
Distribution & $\xi=0.1$ & 1.0001 & 1.0102 & 1.0303 & 1.0605 & 1.1010 & 0.1000 & 0.0100 \\
& $\xi=0.2$ & 1.0002 & 1.0404 & 1.1206 & 1.2424 & 1.4091 & 0.2000 & 0.0400 \\
& $\xi=0.35$ & 1.0003 & 1.1232 & 1.3686 & 1.7516 & 2.3022 & 0.3500 & 0.1225 \\
& $\xi=0.5$ & 1.0005 & 1.2510 & 1.7516 & 2.5650 & 3.8162 & 0.5000 & 0.2500 \\
& $\xi=0.8$ & 1.0008 & 1.6416 & 2.9229 & 5.2548 & 9.4573 & 0.8000 & 0.6400 \\
\hline Uniform & $\xi=0.0$ & 1.0000 & 1.0000 & 1.0000 & 1.0000 & 1.0000 & 0.0000 & 0.0000 \\
Distribution & $\xi=0.1$ & 1.0001 & 1.0102 & 1.0304 & 1.0608 & 1.1017 & 0.1002 & 0.0100 \\
& $\xi=0.2$ & 1.0001 & 1.0405 & 1.1210 & 1.2446 & 1.4170 & 0.2004 & 0.0400 \\
& $\xi=0.35$ & 1.0003 & 1.1236 & 1.3699 & 1.7665 & 2.3674 & 0.3508 & 0.1232 \\
& $\xi=0.5$ & 1.0004 & 1.2519 & 1.7545 & 2.6211 & 4.0781 & 0.5011 & 0.2517 \\
& $\xi=0.8$ & --- & --- & --- & --- & --- & --- & --- \\
\hline
\end{tabular}


Table 2.3: Moments of ensemble averaged distributions (10000 ensembles) used for the one-dimensional chain (256 element long).

\begin{tabular}{|ccccccccc|}
\hline Distribution & Disorder & $\left.<M_{1}\right\rangle$ & $\left\langle M_{2}\right\rangle$ & $\left\langle M_{3}\right\rangle$ & $\left\langle M_{4}>\right.$ & $\left.<M_{5}\right\rangle$ & $\Xi$ & $\Xi^{2}$ \\
& $\xi=0.0$ & 1.0000 & 1.0000 & 1.0000 & 1.0000 & 1.0000 & 0.0000 & 0.0000 \\
& $\xi=0.1$ & 1.0000 & 1.0100 & 1.0299 & 1.0601 & 1.1011 & 0.0999 & 0.0100 \\
Normal & $\xi=0.2$ & 1.0000 & 1.0399 & 1.1196 & 1.2439 & 1.4225 & 0.1998 & 0.04 \\
Distribution & $\xi=0.35$ & 1.0000 & 1.1192 & 1.3598 & 1.7648 & 2.4222 & 0.3456 & 0.1197 \\
& $\xi=0.5$ & 1.0000 & 1.2093 & 1.6491 & 2.4617 & 3.9545 & 0.4579 & 0.2101 \\
& $\xi=0.8$ & 1.0000 & 1.3319 & 2.0893 & 3.6833 & 7.1170 & 0.5767 & 0.3332 \\
\hline \multirow{5}{*}{ Binary } & $\xi=0.0$ & 1.0000 & 1.0000 & 1.0000 & 1.0000 & 1.0000 & 0.0000 & 0.0000 \\
Distribution & $\xi=0.1$ & 1.0000 & 1.0100 & 1.0299 & 1.0599 & 1.1001 & 0.1000 & 0.0100 \\
& $\xi=0.2$ & 1.0000 & 1.0399 & 1.1196 & 1.2409 & 1.4069 & 0.2000 & 0.0400 \\
& $\xi=0.35$ & 1.0000 & 1.1222 & 1.3668 & 1.7494 & 2.3006 & 0.3501 & 0.1226 \\
& $\xi=0.5$ & 1.0000 & 1.2496 & 1.7502 & 2.5665 & 3.8279 & 0.5004 & 0.2506 \\
& $\xi=0.8$ & 1.0000 & 1.6417 & 2.9340 & 5.3080 & 9.6373 & 0.8017 & 0.6442 \\
\hline Uniform & $\xi=0.0$ & 1.0000 & 1.0000 & 1.0000 & 1.0000 & 1.0000 & 0.0000 & 0.0000 \\
Distribution & $\xi=0.1$ & 1.0000 & 1.0100 & 1.0299 & 1.0600 & 1.1006 & 0.1000 & 0.0100 \\
& $\xi=0.2$ & 1.0000 & 1.0399 & 1.1197 & 1.2422 & 1.4134 & 0.2000 & 0.0400 \\
& $\xi=0.35$ & 1.0000 & 1.1223 & 1.3670 & 1.7616 & 2.3605 & 0.3501 & 0.1227 \\
& $\xi=0.5$ & 1.0000 & 1.2499 & 1.7507 & 2.6167 & 4.0775 & 0.5005 & 0.2509 \\
& $\xi=0.8$ & --- & --- & --- & --- & --- & --- & --- \\
\hline
\end{tabular}


Table 2.4: Scaled Coherent Wave Velocity Picking for different particles before and after localization length for a disordered chain with normal distribution (256 element long, 500 ensembles).

\begin{tabular}{|c|c|c|c|c|c|c|c|c|}
\hline Particle Number & Disorder & Average Mass & $5 \%$ Peak & $10 \%$ Peak & $70 \%$ Peak & 90\% Peak & Peak & Zero Crossing \\
\hline \multirow{6}{*}{$130^{t h}$ particle } & $\xi=0.0$ & 1.0000 & 1.0462 & 1.0365 & 1.0002 & 0.9911 & 0.9808 & 0.9560 \\
\hline & $\xi=0.1$ & 1.0000 & 1.0462 & 1.0365 & 1.0002 & 0.9911 & 0.9817 & 0.9560 \\
\hline & $\xi=0.2$ & 1.0000 & 1.0508 & 1.0409 & 1.0032 & 0.9938 & 0.9839 & 0.9560 \\
\hline & $\xi=0.35$ & 1.0000 & 1.0623 & 1.0515 & 1.0100 & 0.9994 & 0.9881 & 0.9525 \\
\hline & $\xi=0.5$ & 1.0000 & 1.0735 & 1.0616 & 1.0155 & 1.0035 & 0.9906 & 0.9449 \\
\hline & $\xi=0.8$ & 1.0000 & 1.0841 & 1.0713 & 1.0211 & 1.0079 & 0.9933 & 0.9328 \\
\hline \multirow{6}{*}{$150^{t h}$ particle } & $\xi=0.0$ & 1.0000 & 1.0402 & 1.0317 & 0.9990 & 0.9910 & 0.9825 & 0.9597 \\
\hline & $\xi=0.1$ & 1.0000 & 1.0419 & 1.0332 & 1.0003 & 0.9920 & 0.9835 & 0.9599 \\
\hline & $\xi=0.2$ & 1.0000 & 1.0464 & 1.0373 & 1.0032 & 0.9946 & 0.9855 & 0.9597 \\
\hline & $\xi=0.35$ & 1.0000 & 1.0574 & 1.0475 & 1.0095 & 0.9998 & 0.9894 & 0.9566 \\
\hline & $\xi=0.5$ & 1.0000 & 1.0678 & 1.0569 & 1.0146 & 1.0036 & 0.9917 & 0.9500 \\
\hline & $\xi=0.8$ & 1.0000 & 1.0782 & 1.0664 & 1.0199 & 1.0076 & 0.9939 & 0.9387 \\
\hline \multirow{6}{*}{$200^{t h}$ particle } & $\xi=0.0$ & 1.0000 & 1.0330 & 1.0258 & 0.9991 & 0.9924 & 0.9856 & 0.9665 \\
\hline & $\xi=0.1$ & 1.0000 & 1.0342 & 1.0271 & 1.0001 & 0.9933 & 0.9862 & 0.9666 \\
\hline & $\xi=0.2$ & 1.0000 & 1.0376 & 1.0303 & 1.0023 & 0.9954 & 0.9878 & 0.9665 \\
\hline & $\xi=0.35$ & 1.0000 & 1.0459 & 1.0380 & 1.0073 & 0.9995 & 0.9910 & 0.9642 \\
\hline & $\xi=0.5$ & 1.0000 & 1.0537 & 1.0450 & 1.0113 & 1.0025 & 0.9929 & 0.9587 \\
\hline & $\xi=0.8$ & 1.0000 & 1.0620 & 1.0526 & 1.0155 & 1.0056 & 0.9947 & 0.9494 \\
\hline \multirow{6}{*}{$220^{t h}$ particle } & $\xi=0.0$ & 1.0000 & 1.0308 & 1.0242 & 0.9992 & 0.9930 & 0.9864 & 0.9685 \\
\hline & $\xi=0.1$ & 1.0000 & 1.0320 & 1.0253 & 1.0000 & 0.9937 & 0.9870 & 0.9686 \\
\hline & $\xi=0.2$ & 1.0000 & 1.0350 & 1.0282 & 1.0020 & 0.9954 & 0.9884 & 0.9685 \\
\hline & $\xi=0.35$ & 1.0000 & 1.0426 & 1.0352 & 1.0066 & 0.9993 & 0.9914 & 0.9665 \\
\hline & $\xi=0.5$ & 1.0000 & 1.0500 & 1.0419 & 1.0105 & 1.0022 & 0.9933 & 0.9619 \\
\hline & $\xi=0.8$ & 1.0000 & 1.0575 & 1.0487 & 1.0142 & 1.0050 & 0.9949 & 0.9542 \\
\hline
\end{tabular}


Table 2.5: Unscaled Coherent Wave Velocity Picking $\left(\sqrt{\tilde{M}_{1}}\right)$ for different particles before and after localization length for a disordered chain with normal distribution (256 element long, 500 ensembles).

\begin{tabular}{|c|c|c|c|c|c|c|c|c|}
\hline Particle Number & Disorder & Average Mass & $5 \%$ Peak & $10 \%$ Peak & $70 \%$ Peak & $90 \%$ Peak & Peak & Zero Crossing \\
\hline \multirow{5}{*}{$130^{t h}$ particle } & $\xi=0.1$ & 1.0000 & 1.0462 & 1.0366 & 1.0002 & 0.9912 & 0.9818 & 0.9560 \\
\hline & $\xi=0.2$ & 1.0000 & 1.0509 & 1.0409 & 1.0033 & 0.9939 & 0.9840 & 0.9560 \\
\hline & $\xi=0.35$ & 1.0022 & 1.0614 & 1.0505 & 1.0091 & 0.9985 & 0.9872 & 0.9515 \\
\hline & $\xi=0.5$ & 1.0274 & 1.0595 & 1.0477 & 1.0022 & 0.9904 & 0.9776 & 0.9322 \\
\hline & $\xi=0.8$ & 1.1581 & 1.0081 & 0.9962 & 0.9496 & 0.9373 & 0.9237 & 0.8668 \\
\hline \multirow{5}{*}{$150^{t h}$ particle } & $\xi=0.1$ & 1.0000 & 1.0420 & 1.0332 & 1.0003 & 0.9921 & 0.9835 & 0.9599 \\
\hline & $\xi=0.2$ & 1.0000 & 1.0465 & 1.0374 & 1.0032 & 0.9946 & 0.9856 & 0.9597 \\
\hline & $\xi=0.35$ & 1.0022 & 1.0564 & 1.0465 & 1.0086 & 0.9989 & 0.9885 & 0.9556 \\
\hline & $\xi=0.5$ & 1.0274 & 1.0539 & 1.0431 & 1.0013 & 0.9905 & 0.9787 & 0.9373 \\
\hline & $\xi=0.8$ & 1.1581 & 1.0026 & 0.9917 & 0.9485 & 0.9370 & 0.9243 & 0.8723 \\
\hline \multirow{5}{*}{$200^{t h}$ particle } & $\xi=0.1$ & 1.0000 & 1.0343 & 1.0271 & 1.0001 & 0.9934 & 0.9862 & 0.9666 \\
\hline & $\xi=0.2$ & 1.0000 & 1.0377 & 1.0304 & 1.0024 & 0.9954 & 0.9879 & 0.9665 \\
\hline & $\xi=0.35$ & 1.0022 & 1.0449 & 1.0370 & 1.0064 & 0.9985 & 0.9901 & 0.9631 \\
\hline & $\xi=0.5$ & 1.0274 & 1.0399 & 1.0313 & 0.9981 & 0.9894 & 0.9799 & 0.9458 \\
\hline & $\xi=0.8$ & 1.1581 & 0.9876 & 0.9788 & 0.9443 & 0.9351 & 0.9250 & 0.8822 \\
\hline \multirow{5}{*}{$220^{t h}$ particle } & $\xi=0.1$ & 1.0000 & 1.0320 & 1.0253 & 1.0000 & 0.9937 & 0.9870 & 0.9686 \\
\hline & $\xi=0.2$ & 1.0000 & 1.0320 & 1.0253 & 1.0000 & 0.9937 & 0.9870 & 0.9685 \\
\hline & $\xi=0.35$ & 1.0022 & 1.0417 & 1.0343 & 1.0057 & 0.9984 & 0.9905 & 0.9654 \\
\hline & $\xi=0.5$ & 1.0274 & 1.0362 & 1.0283 & 0.9972 & 0.9891 & 0.9803 & 0.9490 \\
\hline & $\xi=0.8$ & 1.1581 & 0.9834 & 0.9752 & 0.9431 & 0.9346 & 0.9252 & 0.8867 \\
\hline
\end{tabular}


Table 2.6: Coherent Wave Velocity calculated from the time taken by the pulse to travel a common distance of separation (7 particles/elements) with time calculated in reference to $5 \%, 10 \%, 70 \%, 90 \%$ of the peak value and the peak value of the coherent wave packet.

\begin{tabular}{|c|c|c|c|c|c|c|c|c|}
\hline Particle Number & Disorder & Average Mass & $5 \%$ Peak & $10 \%$ Peak & $70 \%$ Peak & 90\% Peak & Peak & Zero Crossing \\
\hline \multirow{6}{*}{$\begin{array}{l}137^{t h} \text { particle } \\
-130^{t h} \\
\text { particle }\end{array}$} & $\xi=0.0$ & 1.0000 & 1.0135 & 1.0089 & 0.9999 & 0.9954 & 0.9954 & 0.9867 \\
\hline & $\xi=0.1$ & 1.0000 & 1.0143 & 1.0108 & 1.0000 & 0.9970 & 0.9932 & 0.9867 \\
\hline & $\xi=0.2$ & 1.0000 & 1.0167 & 1.0136 & 1.0010 & 0.9980 & 0.9941 & 0.9867 \\
\hline & $\xi=0.35$ & 1.0000 & 1.0227 & 1.0186 & 1.0039 & 0.9996 & 0.9951 & 0.9910 \\
\hline & $\xi=0.5$ & 1.0000 & 1.0284 & 1.0241 & 1.0058 & 1.0006 & 0.9945 & 0.9867 \\
\hline & $\xi=0.8$ & 1.0000 & 1.0319 & 1.0279 & 1.0129 & 1.0088 & 1.0036 & 0.9867 \\
\hline \multirow{6}{*}{$\begin{array}{l}157^{t h} \text { particle } \\
-150^{t h} \\
\text { particle }\end{array}$} & $\xi=0.0$ & 1.0000 & 1.0135 & 1.0089 & 0.9999 & 0.9954 & 0.9954 & 0.9867 \\
\hline & $\xi=0.1$ & 1.0000 & 1.0107 & 1.0082 & 0.9982 & 0.9960 & 0.9930 & 0.9867 \\
\hline & $\xi=0.2$ & 1.0000 & 1.0105 & 1.0080 & 0.9974 & 0.9957 & 0.9928 & 0.9867 \\
\hline & $\xi=0.35$ & 1.0000 & 1.0072 & 1.0054 & 0.9984 & 0.9965 & 0.9948 & 0.9910 \\
\hline & $\xi=0.5$ & 1.0000 & 1.0056 & 1.0041 & 0.9983 & 0.9964 & 0.9940 & 0.9867 \\
\hline & $\xi=0.8$ & 1.0000 & 1.0106 & 1.0072 & 0.9947 & 0.9917 & 0.9880 & 0.9780 \\
\hline \multirow{6}{*}{$\begin{array}{l}227^{t h} \text { particle } \\
-220^{t h} \\
\text { particle }\end{array}$} & $\xi=0.0$ & 1.0000 & 1.0135 & 1.0089 & 0.9999 & 0.9954 & 0.9954 & 0.9910 \\
\hline & $\xi=0.1$ & 1.0000 & 1.0090 & 1.0073 & 0.9969 & 0.9959 & 0.9948 & 0.9867 \\
\hline & $\xi=0.2$ & 1.0000 & 1.0074 & 1.0056 & 0.9968 & 0.9951 & 0.9934 & 0.9867 \\
\hline & $\xi=0.35$ & 1.0000 & 1.0056 & 1.0039 & 0.9957 & 0.9939 & 0.9917 & 0.9867 \\
\hline & $\xi=0.5$ & 1.0000 & 1.0059 & 1.0039 & 0.9968 & 0.9950 & 0.9926 & 0.9867 \\
\hline & $\xi=0.8$ & 1.0000 & 1.0122 & 1.0111 & 1.0062 & 1.0049 & 1.0031 & 0.9867 \\
\hline \multirow{6}{*}{$\begin{array}{l}247^{t h} \text { particle } \\
-240^{t h} \\
\text { particle }\end{array}$} & $\xi=0.0$ & 1.0000 & 1.0089 & 1.0089 & 0.9999 & 0.9999 & 0.9954 & 0.9910 \\
\hline & $\xi=0.1$ & 1.0000 & 1.0087 & 1.0060 & 0.9975 & 0.9966 & 0.9953 & 0.9910 \\
\hline & $\xi=0.2$ & 1.0000 & 1.0073 & 1.0047 & 0.9964 & 0.9952 & 0.9925 & 0.9910 \\
\hline & $\xi=0.35$ & 1.0000 & 1.0041 & 1.0019 & 0.9946 & 0.9928 & 0.9909 & 0.9954 \\
\hline & $\xi=0.5$ & 1.0000 & 1.0017 & 1.0002 & 0.9928 & 0.9916 & 0.9903 & ---- \\
\hline & $\xi=0.8$ & 1.0000 & 0.9937 & 0.9919 & 0.9860 & 0.9846 & 0.9847 & ---- \\
\hline
\end{tabular}





\section{Chapter 3}

\section{Energy Propagation with Distance and Across}

Wavenumbers in a Granular Chain

The hidden harmony is better than the obvious.

Heraclitus

Energy transfer is one of the essentialities during mechanical wave propagation (along with momentum). The diffusive characteristics of energy during wave propagation is already a part of many ongoing investigations. Predicting the energy propagation characteristics in real and wavenumber space through disordered (simplified) model, granular media like chains can assist in understanding the overall properties of wave propagation through real inhomogeneous media like soil; this eventually, can assist in seismic prospecting, non-destructive testing or designing metamaterials.

The effect of disorder on energy propagation is first examined using an impulse propagating in a disordered granular chain, where disorder is given by the standard deviation of the mass distribution of the elements/granules. The use of an ideal one-dimensional granular chain allows isolating longitudinal P-wave mode 
from shear or rotational modes during analysis. From the total energy signals, it is observed that stronger disorder leads to more rapid attenuation of the signal. An ordered granular chain exhibits ballistic propagation of energy and a disordered granular chain exhibits diffusive-like propagation of energy. Disorder causes localization of energy near the source, where the intensity of the localization exhibits a power law like relationship with distance from the source and also increases quadratically with increase in disorder.

Second, the effect of disorder on energy transfer across wavenumbers is examined using a standing sinusoidal wave initial condition (with a specific wavenumber). Disorder causes localization of energy near the particles with the lowest masses in the chain; higher disorder causes a faster decay of higher wavenumbers, whereas lower wavenumbers remain in the system for longer time period. Using the ensembled spatio-spectral energy response from the granular chain, the coefficients of a disorder specific Master Equation are evaluated, which eventually can be used for mean-field macroscopic/continuum analyses.

\subsection{Introduction}

Disorder in granular materials (like soil) can manifest in many ways from grain level to system level (contact disorder, geometrical disorder, asphericity, layering, etc.). All may have an effect on the mechanical wave transmission through the granular material in it's own unique way (for instance, contact disorder due to tiny polydispersity can reduce the mechanical wave speed and the transport of high frequency waves [129]). Knowing these effects can aid us in numerous ways for subsurface exploration or for non-destructive testing of materials. Thus, there is a need to study the effects of disorder individually and hence the focus of this article will only be on mass disorder, for which a 1D granular chain has been chosen so that the P-wave mode is isolated from shear or rotational modes. A mechanical wave propagating through this simplified model 1D granular medium is bound to suffer from multiple scattering. However, regardless of scattering, linear waves preserve some coherence that manifest as intensity correlations ([211]). The results obtained from the chain also represent attributes of both longitudinal P-waves (compressional) and S-waves (shear) in a 3D system as stated in [97]; the frequency filtering effects are very similar to those in a 3D system, as observed in [129]. All the more reason to study energy content and spectral energy response of the propagating wave.

Classical continuum theories and Effective Medium Theory experience difficulty in modeling wave propagation in the intermediate or high frequency range 
because of their inability to resolve the micro-structure of the material (Sect. 1.6.1). Continuum numerical techniques like Finite Element Method (F.E.M), Finite Difference Method (F.D.M), etc. can be used to predict wave propagation characteristics only if the right parameters are used, which are often difficult to find (Sect. 1.7). However, Discrete Element Method (D.E.M), a numerical technique which takes into account the disordered micro-structure of the material and the nonlinear contact forces between the interacting constituent granules of the media. This microscopic description is detailed but also costly so that only small volumes can be modeled. Nevertheless, D.E.M can be used to obtain the parameters of stochastic meso-scale models. These then eventually can be used for continuum, macroscopic wave propagation analyses, hence, paving way towards a statistical micro-informed macroscopic treatment of the problem.

The dynamic wave propagation in a granular chain can be argued as a Markovian process, the initial waveform (displacement/velocity of the particles) and the granular chain properties (pre-compression, sizes/masses of the particles through which the mechanical wave propagates) are sufficient to construct/predict successively the waveform at later time intervals. The transition probability functions of the Markovian processes can be written in the form of Chapman-Kolmogrov equation, one of the versions of this equation is the Master Equation [82]. Hence, a Master Equation can be used to represent the transfer of energy across wavenumbers during mechanical wave propagation.

Sect. 3.2 gives the micro-mechanical model of the granular chain with linearized Hertzian repulsive interaction force acting between the granules; two types of initial condition (impulse propagation condition and standing wave condition; Sect. 3.2.2 and Sect. 3.2.3) are used to analyze energy propagation with distance and across wavenumbers. Sect. 3.2.4 gives a brief description about the mass disorder used in the granular chain. Sect. 3.3 lists the equations used for computing the total energy responses both in real and wavenumber space. Sect. 3.3.2 formulates the Master Equation and proposes the procedures to evaluate the transfer rates of energy across wavenumber space (componenets of the transfer matrix). Results are discussed in Sect. 3.4 and final conclusions are presented in Sect. 3.5.

\subsection{Granular Chain Model}

A granular chain of mesoscopic granules/particles has been modeled using the Hertzian repulsive interaction potential (a good approximation for spherical particles [91]), the repulsive interaction force between adjacent particles $i$ and 
$j$ is ( $j$ can be $i+1$ or $i-1$ ),

$$
\tilde{F}_{(i, j)}=\tilde{\kappa}_{(i, j)} \tilde{\delta}_{(i, j)}^{3 / 2},
$$

where $\tilde{\kappa}_{(i, j)}$ is the dimensional inter-particle contact stiffness, $\tilde{\delta}_{(i, j)}$ is the dimensional dynamic inter-particle overlap and the $3 / 2$ exponent is due to the Hertzian potential. The granular chain has a high pre-confining force $\left(\tilde{\Delta}_{(i, j)}\right)$ which prevents opening and closing of contacts. This assists in modeling mechanical wave propagation across well established granular chains. Assuming an external pre-compressional force $\tilde{F}_{o}$ on the granular chain in mechanical equilibrium, the initial particle overlap is given by

$$
\tilde{\Delta}_{(i, j)}=\left(\frac{\tilde{F}_{o}}{\tilde{\kappa}_{(i, j)}}\right)^{2 / 3} .
$$

The dimensional dynamic overlap is written as $\left.\tilde{\delta}_{(i, j)}=\tilde{\Delta}_{(i, j)}+\tilde{u}^{(i)}-\tilde{u}^{(j)}\right)$, where $\tilde{u}^{(i)}$ and $\tilde{u}^{(j)}$ are the dimensional displacements of the particles $i$ and $j$, respectively. To obtain a non-dimensionalized equation of motion for particles, the physical parameters have to be scaled. The minimum number of scaling parameters required for arriving at a nondimensionalized equation of motion are characteristic mass $\left(\tilde{m}_{o}\right)$, characteristic stiffness $\left(\tilde{\kappa}_{o}\right)$ and a length scale $(\tilde{\ell})$. The length scale could be chosen from a variety of parameters such as the particle size, the driving amplitude or the characteristic initial static overlap $\left(\tilde{\Delta}_{o}=\left(\tilde{F}_{o} / \tilde{\kappa}_{o}\right)^{2 / 3}\right.$; when all $\tilde{\kappa}_{(i, j)}=\tilde{\kappa}_{o}$; uniform contact stiffness; $\tilde{\kappa}_{o}$ is related to $\tilde{m}_{o}$ by Eq. (B.4)). $\tilde{\Delta}_{o}$ is chosen as the length scale for analyses. The nondimensional mass $b^{(i)}=\tilde{m}^{(i)} / \tilde{m}_{o}$, the non-dimensional stiffness is $\kappa_{(i, j)}=\tilde{\kappa}_{(i, j)} / \tilde{\kappa}_{o}$ and the non-dimensional displacement is $u=\tilde{u} / \tilde{\Delta}_{o}$. Without new scaling parameters, this also defines the non-dimensional time $t=\tilde{t} / \tilde{t}_{c}$ where

$$
\tilde{t}_{c}=\sqrt{\frac{\tilde{m}_{o}}{\tilde{\kappa}_{o} \tilde{\Delta}_{o}^{1 / 2}} .}
$$

and the non-dimensional repulsive interaction force

$$
F_{(i, j)}=\frac{\tilde{t}_{c}^{2}}{\tilde{m}_{o} \tilde{\Delta}_{o}} \tilde{F}_{(i, j)} .
$$

The non-dimensional equation of motion for particle $i$ is now given by

$$
\begin{aligned}
b^{(i)} \frac{\mathrm{d}^{2} u^{(i)}}{\mathrm{d} t^{2}} & =F_{(i-1, i)}-F_{(i, i+1)} \\
& =\kappa_{(i-1, i)}\left[\Delta_{(i-1, i)}-\left(u^{(i)}-u^{(i-1)}\right)\right]^{3 / 2}-\kappa_{(i, i+1)}\left[\Delta_{(i, i+1)}-\left(u^{(i+1)}-u^{(i)}\right)\right]^{3 / 2}
\end{aligned}
$$


Eq. (3.5) can be solved numerically using the Verlet integration scheme, it can be used for analyses related to nonlinear dynamics of particles (Hertzian). The non-dimensional stiffness $\left(\kappa_{(i, j)}\right)$ and the initial overlap are given by (Appendix B; [91])

$$
\kappa_{(i, j)}=\sqrt{\frac{2}{b^{(i)}+b^{(j)}}}\left(b^{(i)} b^{(j)}\right)^{1 / 6} \quad \text { and } \quad \Delta_{(i, j)}=\kappa_{(i, j)}^{-2 / 3},
$$

\subsubsection{Linearized Equation of Motion}

$F_{(i, j)}=\kappa_{(i, j)} \delta_{(i, j)}^{3 / 2}$ can be expanded around the initial overlap $\Delta_{(i, j)}$ as

$$
F_{(i, j)}=\kappa_{(i, j)} \Delta_{(i, j)}^{3 / 2}+\frac{3}{2} \kappa_{(i, j)} \Delta_{(i, j)}^{1 / 2}\left(\delta_{(i, j)}-\Delta_{(i, j)}\right)+\frac{3}{8} \kappa_{(i, j)} \Delta_{(i, j)}^{-1 / 2}\left(\delta_{(i, j)}-\Delta_{(i, j)}\right)^{2}+\ldots
$$

If the amplitudes of displacement $\left(u^{(i)}\right)$ are small during mechanical wave propagation, the nonlinear terms can be ignored and

$$
F_{(i, j)}=\kappa_{(i, j)} \Delta_{(i, j)}^{3 / 2}-\frac{3}{2} \kappa_{(i, j)} \Delta_{(i, j)}^{1 / 2}\left(u^{(j)}-u^{(i)}\right) .
$$

Hence, the linearized equation of motion for particle $i$ becomes

$$
\begin{aligned}
b^{(i)} \frac{\mathrm{d}^{2} u^{(i)}}{\mathrm{d} t^{2}} & =\kappa_{(i-1, i)} \Delta_{(i-1, i)}^{1 / 2}\left[\Delta_{(i-1, i)}-\frac{3}{2}\left(u^{(i)}-u^{(i-1)}\right)\right] \\
& -\kappa_{(i, i+1)} \Delta_{(i, i+1)}^{1 / 2}\left[\Delta_{(i, i+1)}-\frac{3}{2}\left(u^{(i+1)}-u^{(i)}\right)\right],
\end{aligned}
$$

which can eventually be written as

$$
b^{(i)} \frac{\mathrm{d}^{2} u^{(i)}}{\mathrm{d} t^{2}}=\frac{3}{2} \kappa_{(i, i+1)}^{2 / 3}\left(u^{(i+1)}-u^{(i)}\right)-\frac{3}{2} \kappa_{(i-1, i)}^{2 / 3}\left(u^{(i)}-u^{(i-1)}\right) .
$$

Eq. (3.10) results in $N$ equations which are assembled in a matrix form:

$$
\mathbf{M} \frac{\mathrm{d}^{2} \mathbf{u}}{\mathrm{d} t^{2}}=\mathbf{K u}
$$

where $\mathbf{M}$ is a diagonal matrix with $b^{(1)}, b^{(2)}, b^{(3)} \ldots . . b^{(N)}$ as diagonal elements, $\mathbf{K}$ has $-\frac{3}{2}\left(\kappa_{(i+1, i)}^{2 / 3}+\kappa_{(i-1, i)}^{2 / 3}\right)$ as diagonal, $\frac{3}{2} \kappa_{(i+1, i)}^{2 / 3}$ as superdiagonal and $\frac{3}{2} \kappa_{(i-1, i)}^{2 / 3}$ as 
subdiagonal elements, other elements of $\mathbf{K}$ are $0 . \mathbf{u}$ is the displacement vector containing $u^{(i)}$ as the elements. Assuming $\mathbf{A}=-\mathbf{M}^{-1} \mathbf{K}$, Eq. (3.11) becomes

$$
\frac{\mathrm{d}^{2} \mathbf{u}}{\mathrm{d} t^{2}}=-\mathbf{A} \mathbf{u} \text {. }
$$

Using an ansatz $\mathbf{u}=\mathbf{u}^{\prime} \exp ^{I \omega t}$, Eq. (3.12) becomes an eigenvalue problem

$$
\mathbf{A u}=\omega^{2} \mathbf{u} .
$$

The eigenvalues $\omega_{j}^{2}\left(\omega_{j}\right.$ are the natural frequencies) and the eigenvectors $\mathbf{s}_{(j)}$ of the matrix A represent the eigendomain of the dynamic granular chain. The set of eigenvectors $\left(\mathbf{s}_{(j)}\right)$ can be orthonormalized by the condition

$$
\mathbf{s}_{(i)}^{\mathrm{T}} \mathbf{M s}_{(j)}=\delta_{i j},
$$

where $\delta_{i j}$ is the Kronecker delta symbol. A matrix $\mathbf{S}$ is constructed using $\mathbf{s}_{(j)}$ as columns and arranged in such a manner such that their corresponding $\omega_{j}$ are in increasing order. $\mathbf{S}$ is an eigenbasis matrix and can be used for projecting $u$ into eigenspace by the relation

$$
\mathbf{z}=\mathbf{S}^{-1} \mathbf{u}
$$

where $\mathbf{z}$ is the displacement amplitude (per eigenmode) in the eigenspace. Using the transformation $\mathbf{S}^{-1} \mathbf{A S}=\mathbf{G}$, where $\mathbf{G}$ is a diagonal matrix with $\omega_{j}^{2}$ as the diagonal elements, Eq. (3.12) becomes

$$
\frac{\mathrm{d}^{2} \mathbf{z}}{\mathrm{d} t^{2}}=-\mathbf{G z}
$$

The solution of this equation is given by (in eigenspace and real space, respectively)

$$
\mathbf{z}(t)=\mathbf{C}^{(1)} \mathbf{a}+\mathbf{C}^{(2)} \mathbf{b} \quad \text { or, } \quad \mathbf{u}(t)=\mathbf{S} \mathbf{C}^{(1)} \mathbf{a}+\mathbf{S C}^{(2)} \mathbf{b},
$$

where $\mathbf{C}^{(1)}$ is a diagonal matrix with $\sin \left(\omega_{j} t\right)$ as diagonal elements, $\mathbf{C}^{(2)}$ is also a diagonal matrix with $\cos \left(\omega_{j} t\right)$ as diagonal elements, $\mathbf{a}$ and $\mathbf{b}$ are vectors which are determined from initial conditions $\mathbf{u}_{o}$ (initial displacement vector) and $\mathbf{v}_{o}$ (initial velocity vector).

$$
\mathbf{a}=\mathbf{H}^{-1} \mathbf{S}^{-1} \mathbf{v}_{o} \quad \text { and } \quad \mathbf{b}=\mathbf{S}^{-1} \mathbf{u}_{o},
$$

where $\mathbf{H}$ is a diagonal matrix with $\omega_{j}$ as the diagonal elements. There are $N+2$ particles in the granular chain with $0^{t h}$ and $(N+1)^{t h}$ particles as the boundaries of the chain such that $u^{(0)}=0$ and $u^{(N+1)}=0$. Two different types of initial conditions have been used for different types of analyses in the upcoming sections, impulse propagation and standing wave analysis. 


\subsubsection{Impulse Propagation Condition}

The initial condition for impulse propagation requires $\mathbf{u}_{o}$ and $\mathbf{v}_{o}$ to be

$$
u^{(i)}(t=0)=0, \quad v^{(i \neq n)}(t=0)=0, \quad v^{(n)}(t=0)=v_{o},
$$

where $n$ is the particle number to which impulse is imparted. $v_{o} \ll 1$ (initial particle overlap $\Delta_{o}$ ) to avoid opening and closing of contacts or to maintain the validity of the linearized equations of motion (Sect. 3.2.1). The first and the center particles in a granular chain have been imparted with impulse for further analyses in Sect. 3.4.1. Eq. (3.19) is used to get

$$
\mathbf{a}=\mathbf{H}^{-1} \mathbf{S}^{-1} \mathbf{v}_{o}, \quad \mathbf{b}=0 .
$$

Hence, the displacement and velocity of particle $i$ becomes

$$
\mathbf{u}=\mathbf{S} \mathbf{C}^{(1)} \mathbf{H}^{-1} \mathbf{S}^{-1} \mathbf{v}_{\boldsymbol{O}} \quad \text { and } \quad \mathbf{v}=\mathbf{S} \mathbf{C}^{(2)} \mathbf{S}^{-1} \mathbf{v}_{o} ;
$$

which is also written as:

$$
u^{(i)}(t)=v_{o} \sum_{j=1}^{N} \frac{S_{i j} S_{n j} \sin \left(\omega_{j} t\right)}{\omega_{j}} \quad \text { and } \quad v^{(i)}(t)=v_{o} \sum_{j=1}^{N} S_{i j} S_{n j} \cos \left(\omega_{j} t\right) .
$$

\subsubsection{Standing Wave Condition}

For studying standing waves in the chain, an initial sinusoidal waveform is given to the chain in the form of $\mathbf{u}_{o}=u_{o} \sin \left(\mathscr{N} \frac{2 \pi i}{N+1}\right)$ (where $\mathscr{N}$ specifies the particular tone of the standing wave). $u_{o} \ll 1$ (initial particle overlap $\Delta_{o}$ ) to avoid opening and closing of contacts or to maintain the validity of the linearized equations of motion (Sect. 3.2.1). $\mathbf{v}_{o}=0$. $\mathbf{a}$ and $\mathbf{b}$ are given as:

$$
\mathbf{a}=\mathbf{H}^{-1} \mathbf{S}^{-1} \mathbf{v}_{o}=0 \text { and } \mathbf{b}=\mathbf{S}^{-1} \mathbf{u}_{o} .
$$

Hence, the displacement and velocity of particle $i$ becomes:

$$
\mathbf{u}=\mathbf{S} \mathbf{C}^{(2)} \mathbf{S}^{-1} \mathbf{u}_{o} \text { and } \mathbf{v}=-\mathbf{S} \mathbf{H} \mathbf{C}^{(1)} \mathbf{S}^{-1} \mathbf{u}_{o} .
$$

which is also written as:

$$
\begin{aligned}
& u^{(i)}(t)=\epsilon \sum_{j=1}^{N} S_{i j} \cos \left(\omega_{j} t\right) \sum_{p=1}^{N} S_{p j} \sin \left(\mathscr{N} \frac{2 \pi i}{N+1}\right), \\
& v^{(i)}(t)=-\epsilon \sum_{j=1}^{N} \omega_{j} S_{i j} \sin \left(\omega_{j} t\right) \sum_{p=1}^{N} S_{p j} \sin \left(\mathscr{N} \frac{2 \pi i}{N+1}\right) .
\end{aligned}
$$


Impulse Propagation:

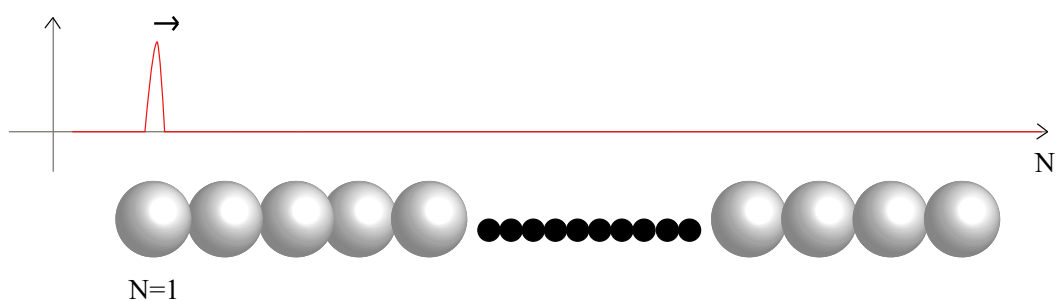

Standing Wave:

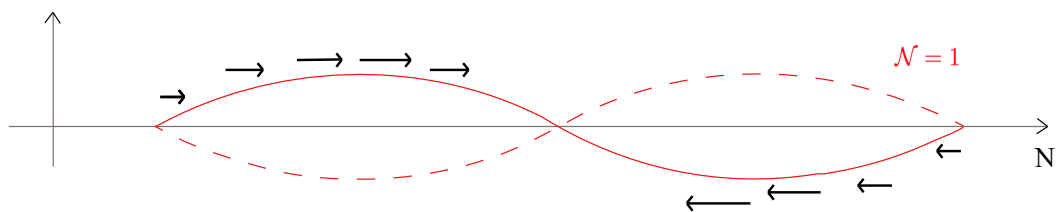

Figure 3.1: Impulse and Standing Wave initial conditions for a granular chain.

\subsubsection{Mass Disorder/Disorder Parameter $(\xi)$ \& Ensembles}

$b^{(1)}, b^{(2)}, b^{(3)}, \ldots b^{(N)}$, which are the diagonal elements of the mass matrix $\mathbf{M}$, have been selected from a normal distribution $f^{(n)}(b)=\frac{1}{\xi \sqrt{2 \pi}} e^{-\frac{(b-1)^{2}}{2 \xi^{2}}}$, the standard deviation $(\xi)$ quantifies the disorder parameter of the granular chain and the scaled average of the distribution is 1 .'A similar model has been used previously in $[97,98,177,178]$ for various wave propagation analyses (Section 2.2.6). It was observed in $[98,177]$ that the shape of the disorder probability (binary, normal, uniform or any other distribution) produces quantitatively similar wave propagation effects (frequency filtering, attenuation or mechanical wave velocities), up to a certain strength of disorder. The physical quantities (e.g. displacement, velocity, total energy, etc.) of multiple realisations of granular chains with a particular disorder parameter are averaged to obtain ensembled quantities, depicted by angular brackets \langle\rangle . 


\subsection{Energy Evolution}

For calculating the Kinetic Energy of individual elements / particles we define the matrix $K E_{p q}$, where,

$$
\mathbf{K E}=\frac{1}{2} \mathbf{M}[\mathbf{v} \otimes \mathbf{v}]=\frac{1}{2} \mathbf{M v v}^{\mathbf{T}}
$$

The Kinetic Energy of individual elements/particles are the diagonal elements of the matrix KE, i.e $K E_{P P}$ (capital letters $P P$ have been used as indices to denote the diagonal elements to avoid confusion with $p p$ which implies summation of the diagonal elements i.e the trace of the matrix KE).

$$
K E^{(p)}(t)=K E_{P P}=\delta_{p P} \delta_{q P} K E_{p q}=\frac{1}{2} b^{(p)}\left(v^{(p)}(t)\right)^{2} .
$$

The Total Kinetic Energy is the trace of the matrix KE, i.e $K E_{p p}$,

$$
K E T(t)=K E_{p p}=\delta_{p q} K E_{p q}=\frac{1}{2} \sum_{p} b^{(p)}\left(v^{(p)}(t)\right)^{2}
$$

The Potential Energy of individual elements is basically the energy stored in the form of compression at the contacts of an element, it arises from the forces $\left(F^{(p)}\right)$ exerted by other elements in contact with the element whose potential energy is being examined. (For details please refer to Appendix D). For the Potential Energy, as well, we define the matrix

$$
\mathbf{P E}=-\frac{1}{2} \mathbf{K}[\mathbf{u} \otimes \mathbf{u}]=-\frac{1}{2} \mathbf{K u \mathbf { u } ^ { \mathbf { T } }}=-\frac{1}{2} \mathbf{F} \mathbf{u}^{\mathbf{T}} \quad \text { with } \quad \mathbf{F}=\mathbf{K u},
$$

where $\mathbf{K}$ is the stiffness matrix (Eq. (3.11)). The Potential Energy for individual elements are the diagonal elements of the matrix $P E_{p q}$,

$$
P E^{(p)}(t)=P E_{P P}=\delta_{p P} \delta_{q P} P E_{p q}=-\frac{1}{2} F^{(p)}(t) u^{(p)}(t) .
$$

The Total Potential Energy is the trace of the matrix $P E_{p q}$, i.e $P E_{p p}$,

$$
P E T(t)=P E_{p p}=\delta_{p q} P E_{p q}=-\frac{1}{2} \sum_{p} F^{(p)}(t) u^{(p)}(t) .
$$

The Total Energy of individual particles is the sum of its kinetic and potential energies. Using Eq. (3.26) and Eq. (3.29),

$$
\mathbf{T E}=\mathbf{K E}+\mathbf{P E}=K E_{p q}+P E_{p q} .
$$


Using Eq. (3.27) and Eq. (3.30),

$$
T E^{(p)}(t)=T E_{P P}=\delta_{p P} \delta_{q P} T E_{p q}=\frac{1}{2}(b(p))\left(v^{(p)}(t)\right)^{2}-\frac{1}{2} F^{(p)}(t) u^{(p)}(t) .
$$

We calculate the Energies (Potential Energy, Kinetic Energy and hence, Total Energy) relative to the initial pre-compressed state so that only the energy associated with wave propagation across the elements is taken into account. The equations (3.27), (3.28), (3.30) \& (3.31) are derived in Appendix D.

The center of total energy is defined as [7]

$$
R(t)=\frac{1}{T E_{\text {tot }}} \sum_{p=1}^{N} p T E^{(p)}(t) \quad \text { with } \quad T E_{\text {tot }}=\sum_{p=1}^{N} T E^{(p)}(t) .
$$

The mean squared width of the total system propagating and trapped wave is [7]

$$
r^{2}(t)=\frac{1}{T E_{t o t}} \sum_{p=1}^{N}(p-R(t))^{2} T E^{(p)}(t) .
$$

\subsubsection{Total Energy in the Wavenumber Domain}

TE or $T E_{p q}$ is in real space, to transform it into wavenumber space $\hat{\mathbf{T E}}$ or $\hat{T E} E_{k m}$ ( $k$ and $m$ being rows and columns in wavenumber space), there is a need of change of basis as $\hat{\mathbf{T E}}=\mathbf{F} \mathbf{T E} \mathbf{F}^{-1}$, where $\mathbf{F}$ is the discrete Fourier transform matrix ${ }^{1}$, it can be used to calculate Fourier transform of vectors such that $\hat{\mathbf{u}}=\mathbf{F u}$ and $\hat{\mathbf{v}}=\mathbf{F v}$, where $\hat{\mathbf{u}}$ and $\hat{\mathbf{v}}$ are displacement and velocity vectors in wavenumber space, respectively. Hence,

$$
\hat{\mathbf{T E}}=\mathbf{F} \mathbf{T E} \mathbf{F}^{-1} \text {. }
$$

The diagonal elements of this matrix yields the total energy in wavenumber space per wavenumber $k$ :

$$
T E^{(k)}(t)=\hat{T E_{K K}}=\delta_{k K} \delta_{m K} \hat{T E_{k m}} .
$$

\footnotetext{
${ }^{1} \mathbf{F}$ can be computed numerically as $\operatorname{dftmt}(\mathrm{x})$ matrix in Matlab, where $\mathrm{x}$ is the size of the square matrix $\mathbf{F}$.
} 


\subsubsection{Numerical Master Equation}

A Master Equation can be an efficient tool in analysing the criss-cross transfer of energy across different wavenumbers. The transfer of spatio-spectral energy is formulated as

$$
\frac{\mathrm{d} e^{(r)}(t)}{\mathrm{d} t}=Q_{R R}(t) e^{(r)}(t)+\sum_{r \neq s} Q_{r s}(t) e^{(s)}(t),
$$

$Q_{R R}$ depicts the energy loss (rate) from a particular wavenumber band $r$, which eventually gets transferred to all other wavenumber bands $s \neq r$. $Q_{r s}$ quantifies the transfer rates of energy back to the wavenumber band $r$ from $s$. $e^{(r)}(t)$ is the $r^{\text {th }}$ component of the binned energy vector $\mathbf{e}$ in wavenumber space. $\mathbf{e}$ is constructed by binning the spatio-spectral energy responses $\left(T E^{(k)}(t)\right)$. The binning is done by

$$
e^{(r)}(t)=\sum_{k=r-\Delta k / 2}^{k=r+\Delta k / 2} T E^{(k)}(t),
$$

where $\Delta k$ is the bandwidth of the bin and $r$ is the central wavenumber.

The total energy is conserved, hence,

$$
\sum_{r=1}^{B} e^{(r)}(t)=\sum_{k} T E^{(k)}(t),
$$

where $B$ is the total number of bins assigned in wavenumber space. The binned spectral energy is normalized as

$$
\hat{e}^{(r)}(t)=\frac{e^{(r)}(t)}{\sum_{r=1}^{B} e^{(r)}(t)}
$$

The coefficients $Q_{R R}$ and $Q_{r s}$ can be assigned as the diagonal and nondiagonal elements of the transfer matrix $Q$, respectively. Eq. (3.38) is thereby written in the scaled matrix form as

$$
\frac{\mathrm{d} \hat{\mathbf{e}}}{\mathrm{d} t}=\mathbf{Q} \hat{\mathbf{e}}
$$

\section{Computing the elements of matrix $\mathbf{Q}$}

The elements of the matrix $\mathbf{Q}$ can be computed numerically using the boundary condition in Sect. 3.2.3, a standing wavemode $k$ belonging to a particular 
wavenumber band $r$ can be agitated in the form of a sinus initial condition (Eq. (3.25)).

Procedure 1: The energy decay of a particular wavenumber (band) as the energy spreads to other bands can be fitted by $\left\langle\hat{e}^{(r)}(t)\right\rangle=1-Q_{R R} t$ for small time intervals (the brackets $\langle\ldots\rangle$ indicate that ensembling is used to improve the quality of the fit); similarly, $Q_{r s}$ is determined by fitting $\left\langle e^{(s)}(t)\right\rangle=Q_{r s} t$.

Procedure 2: The energy decay from normalized $e_{o}=1$ can be fitted by $\left\langle e^{(r)}(t)\right\rangle=$ $e^{-x_{1} t}+y_{1}$, where $x_{1}=Q_{R R}$ (obtained by comparing the coefficients after Taylor expansion) and $y_{1}$ represents the long term saturation value; similarly, $Q_{r s}$ is determined by fitting $\left\langle e^{(s)}(t)\right\rangle=x_{2}\left(1-e^{-t / y_{2}}\right)$, where $Q_{r s}=x_{2} / y_{2}$ (obtained by comparing the coefficients after Taylor expansion) and $x_{2}$ represents the long term saturation value. In the following we apply Procedure 1 , to obtain $\mathbf{Q}$ in Fig. 3.15 and Procedure 2 to obtain $\mathbf{Q}$ in Fig. 3.17 (b).

\subsection{Results}

An $N$ particles long granular chain has been used with Impulse and Standing Wave initial conditions for analyses. Sect. 3.4.1 deals with propagation of energy in a granular chain and the associated energy transfer. Sect. 3.4.2 deals with the analyses associated with energy transport between different wavenumbers.

\subsubsection{Energy Propagation with Distance}

Two types of impulse initial conditions are used. In one of the systems, the first particle $(N=1)$ has been imparted with initial velocity $v_{o}$. In the other system, the center particle has been imparted with $v_{o}$. Eq. (3.34) and Eq. (3.35) have been used for diffusion analyses associated with the impulse propagating in the granular chain.

\section{First Particle Excitation}

Here, an $N=1024$ particles long granular chain is used. Particle $p=1$ is imparted with $v_{o}=0.01$. The time step for the computation is $\Delta t=0.1250$ and the maximum time evaluated is $t_{\max }=1024$, chosen in order to avoid reflection 

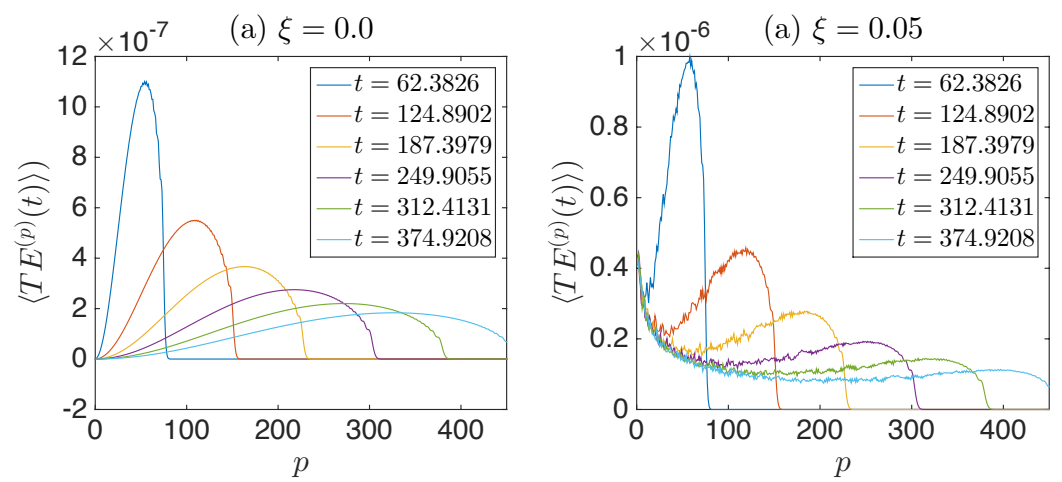

Figure 3.2: Ensembled energy signal at different instances of time for (a) ordered and (b) disordered chains.

of the incident wave from the boundary. Figs. 3.2 and 3.3 display the ensembled total energy signal of four different disordered chains $\xi=0$ (Fig. 3.2(a)), $\xi=0.05$ (Fig. 3.2(b)), $\xi=0.1$ (Fig. 3.3(a)), $\xi=0.35$ (Fig. 3.3(b)). 500 different realizations of chains are used for ensembling. It is observed that there are two peaks in the energy signal for all instances of time shown, irrespective of disorder except for the ordered chain (Fig. 3.2(a)). The first peak is due to weak localization, a coherent backscattering effect during wave propagation near the source (Sect. 1.5) and the second peak is due to the propagating coherent wavefront (Fig. 3.3(a)). The ordered chain does not exhibit the weak localization peak because of absence of disorder. Higher $\xi$ shows a more rapid drop of the propagating coherent wavefront.

Moreover, the weak localization peak decays with distance from the source, the total energy signals at all time instances collapse along this curve except the propagating wavefront which propagates along this long time limit decay curve.

Fig. 3.4 is the log plot of the decay curve associated with weak localization for chains (ensembled total energy signal at $t=875$ per particle; measurements are taken by limiting the space interval $(p \leq 800)$ to avoid propagating wavefront), a power law relationship can be observed from the figure. It is observed that the rate of decay increases with increasing disorder parameter $\xi$ of the chain, indicating a stronger weak localization decay curve with increase in disorder. Fig. 3.5(a) shows the total energy signal of particle $p=1$ (source) with time. The figure shows that after the initial impulse, the energy of the source 

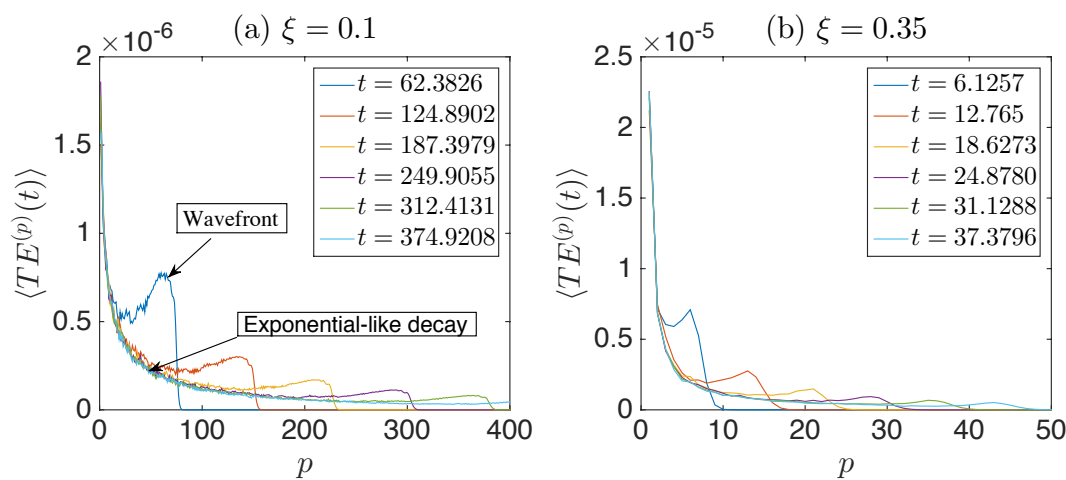

Figure 3.3: Ensembled energy signal at different instances of time for moderate and strongly disordered chains.

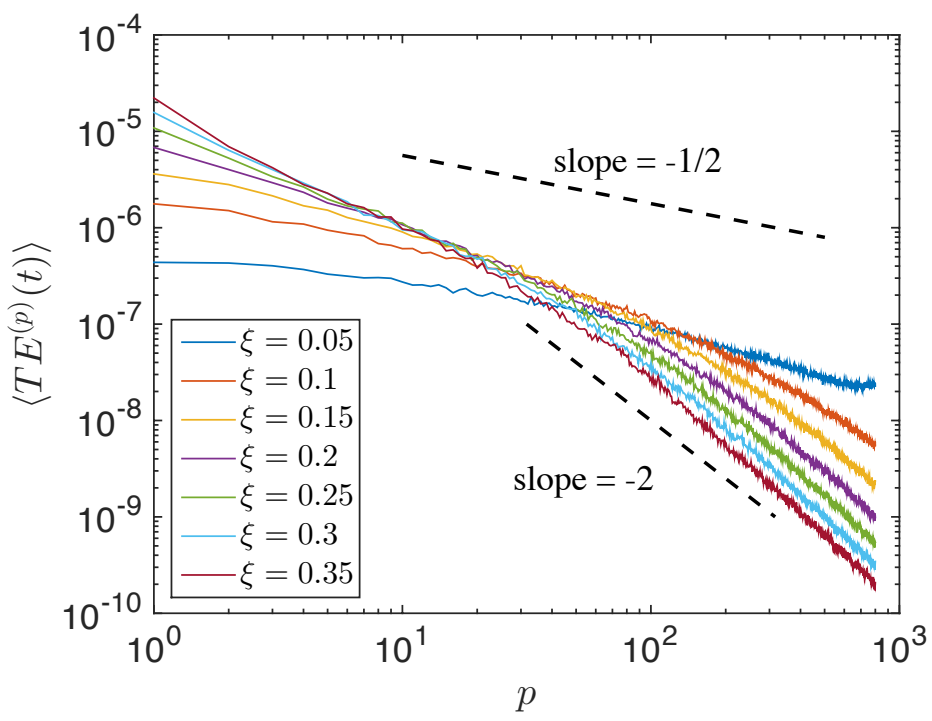

Figure 3.4: Power law relationship of the weak localization decay curve for different $\xi$, at later time $t \cong 875$. 

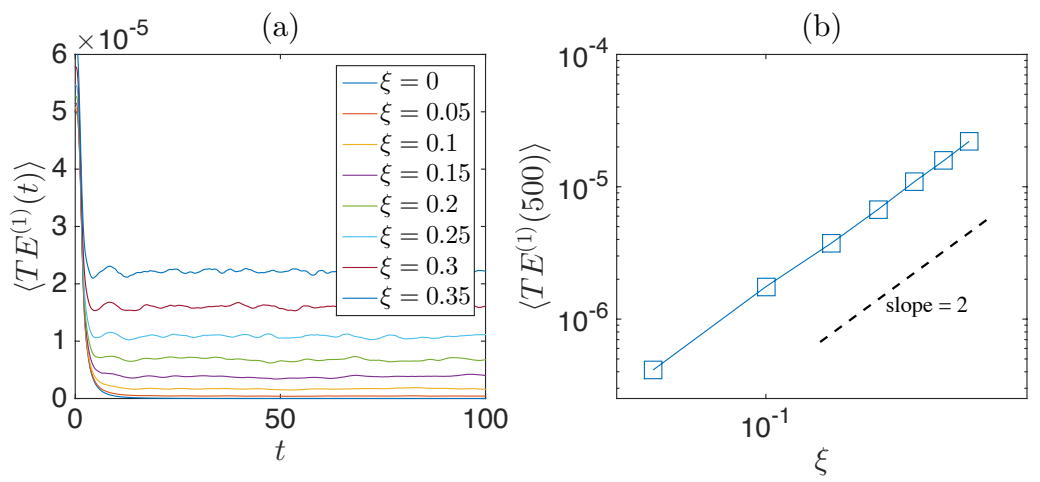

Figure 3.5: (a)Total Energy (averaged over 500 ensembles) of $p=1$ particle with time for chains with different $\xi$. (b)Almost constant residual energy localized in the first particle measured at $t \cong 500$

particle decays and becomes constant with very little fluctuations. This residual energy of the particle increases with increasing $\xi$, Fig. 3.5(b) shows a power law relationship of 2 between the disorder $\xi$ and the $T E$ of the first particle at long time $t=500$.

\section{Diffusion}

The system used in the previous section $(N=1024$ particles long granular chain with $v_{o}=0.01$ imparted to the $1^{\text {st }}$ particle) is used in this sub-section as well. Eq. (3.34) is used to compute $\langle R(t)\rangle$ averaged over 500 ensembles. $\langle R(t)\rangle$ gives the averaged propagation of the center of energy, as plotted in Fig. 3.6. It shows that initially the center of energy does not propagate (as shown in the inset), during which the initial high frequency impulse is self-demodulated [203] by the granular chain (in contrast to a Gaussian pulse [7]). After this short time interval, the center of energy propagates with the same speed for different disorder parameters. $\xi=0.0$ has linear (ballistic) propagation of center of energy whereas, $\xi>0$ leads to nonlinear propagation of the center of energy with propagation speed decreasing with increasing time (Sect. 2.4 [177]). Stronger disorder yields smaller propagation speed with increase in time. Unlike $\xi=0.0$, higher $\xi$ result in the center of energy becoming confined in a finite space and this confinement space is smaller for stronger disorder. This occurs because 
$R(t)$ takes into account both the weak localization occurring close to the source and the propagating wavefront. The mean squared width of the energy during

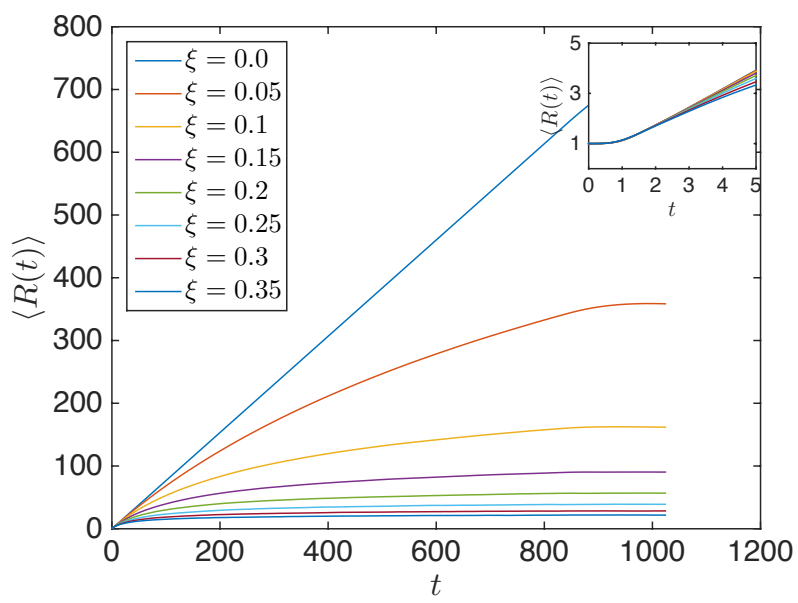

Figure 3.6: Position of the center of energy $(\langle R(t)\rangle)$ during impulse propagation for granular chains with different $\xi$.

impulse propagation $r^{2}(t)$ is computed using Eq. (3.35), averaged over 500 realizations. Fig. 3.7 displays $\left\langle r^{2}(t)\right\rangle$ for granular chains with different $\xi$. It is observed that for an impulse response, the energy propagation is slightly superballistic for low disorder parameters (e.g. $\xi=0.05$ ) and gets nonlinear towards superdiffusive, diffusive and then subdiffusive for high disorder parameters.

\section{Center Particle Excitation}

In order to ensure that the localization of energy during wave propagation is occurring near the source and is not a boundary effect, the effect of a different initial condition on impulse propagation in a granular chain is examined, $p=$ $1025^{\text {th }}$ particle of $N=2049$ particles long granular chain is imparted with $v_{o}=$ 0.01 . The time step for the computation $\Delta t=0.2501$ and $t_{\max }=1024$. Fig. 3.8 shows the total energy signal per particle at a particular instance of time $t \cong 750$ before the wave reach the end of the system for $\xi=0.1$ (Fig. 3.8(a)) and $\xi=0.35$ (Fig. 3.8(b)). It can be observed that the energy is localized around the source 


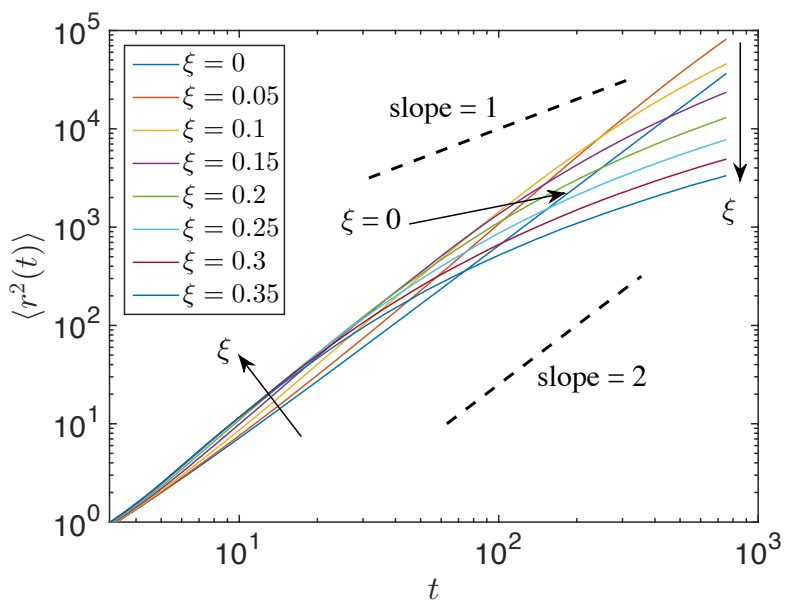

Figure 3.7: $\left\langle r^{2}(t)\right\rangle$ for different $\xi$ in $\log$-log scale. Line is a guide to the eye. Arrows indicate the trend for $\xi>0$.

(the middle particle) and the two propagating wavefronts are moving in the opposite direction. The figure is symmetric around the center particle, which means we are in linear regime and tension and compression wave have same speed.

\subsubsection{Energy Propagation across Wavenumbers: Master Equa- tion for Spectral Energy}

With the goal to understand the evolution of standing waves in time, an initial sinusoidal waveform $\left(\mathbf{u}_{o}=u_{o} \sin \left(\mathscr{N} \frac{2 \pi i}{N+1}\right)\right.$; Sect. 3.2.3) is imparted to an $N=256$ particles long granular chain with $u_{o}=0.01$ and different $\xi$. The evolution of displacement and energy responses of particles/elements are then analyzed. Fig. 3.9 shows the evolving displacement of particles for the $\mathscr{N}=1$ standing wave in an ordered $(\xi=0.0)$ and a disordered chain $(\xi=0.35)$. Fig. 3.9(a) displays the particles (color signifies the displacement amplitude) performing a standing wave motion $(\xi=0.0)$. However, in Fig. 3.4.2(b), it is observed that particles exhibit a perturbed standing wave motion of fluctuating low amplitude (color) in addition with traveling waves, clearly indicating that the disorder in 

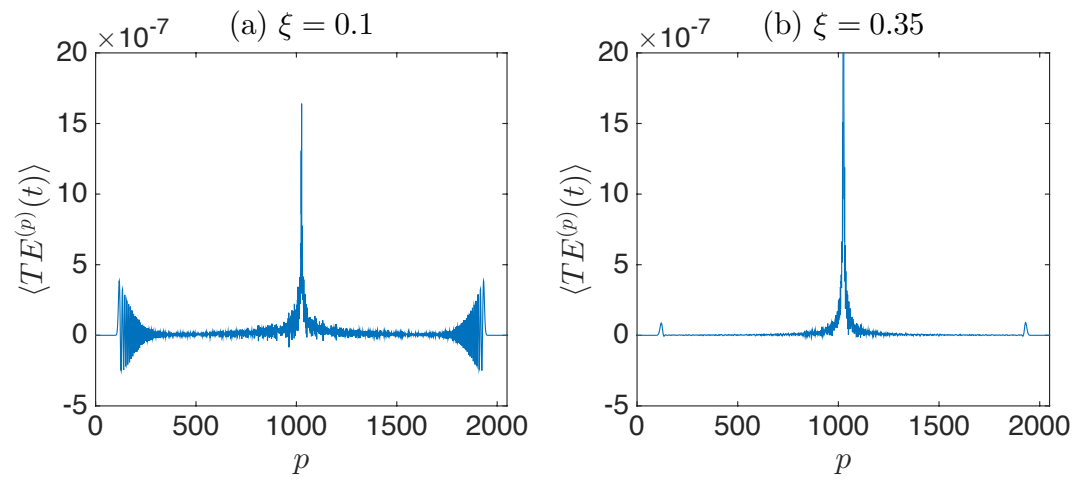

Figure 3.8: Center pulse $(p=1025)$ is imparted with $v_{o}=0.01$ initially towards right. The total Energy signal is averaged over 500 ensembles at $t \cong 750$.

(a) $\xi=0.0$

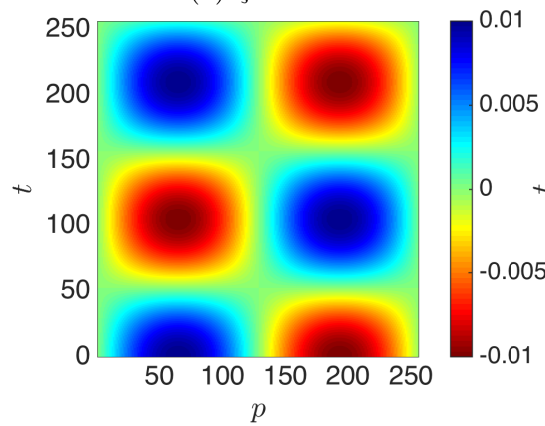

(b) $\xi=0.35$

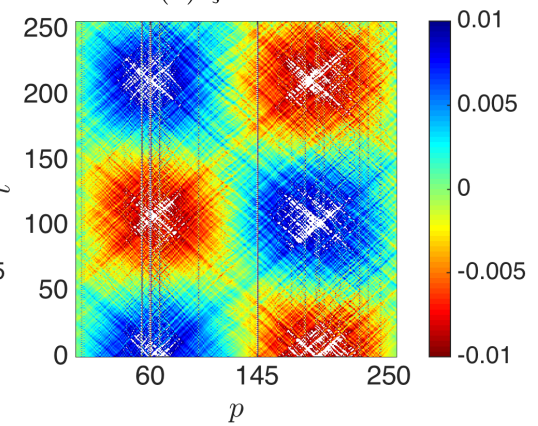

Figure 3.9: (a) $u^{(p)}(t)\left(p=1\right.$ to 256) of an ordered granular chain $(\xi=0.0)$. (b) $u^{(p)}(t)$ ( $p=1$ to 256) of a disordered granular chain $(\xi=0.35)$. 
the chains is disrupting the standing wave motion. It can also be observed that there are few localized high amplitude displacements shown by certain particles like $p=60$ and $p=145$; these particles are the lowest and the third lowest mass particles in the granular chain in addition to being close to the peaks of the standing wave.
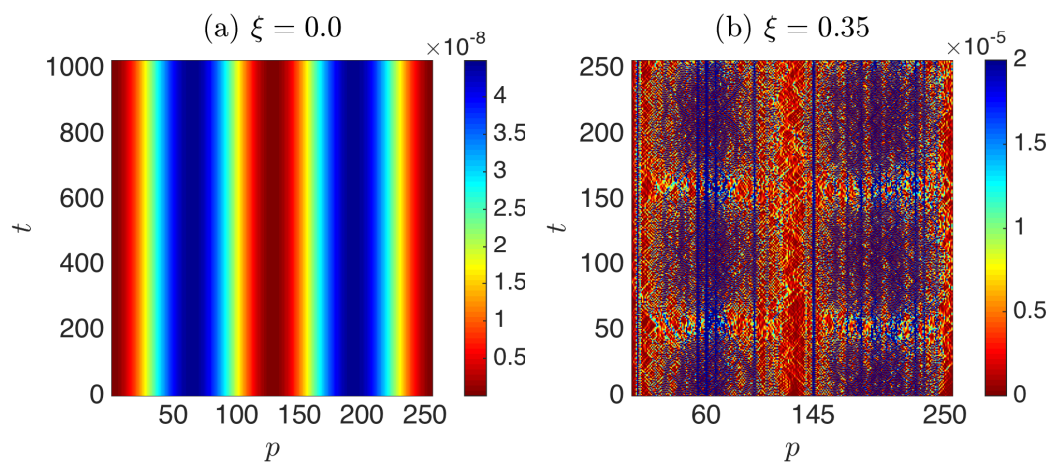

Figure 3.10: (a) $T E^{(p)}(t)$ ( $p=1$ to 256) of an ordered granular chain $(\xi=0.0)$. $T E^{(p)}(t)$ ( $p=1$ to 256) of a disordered granular chain $(\xi=0.35)$.

Fig. 3.10 (a) shows the total energy of the particles for the ordered ( $\xi=$ $0)$ and disordered $(\xi=0.35)$ granular chain (color represents the amplitude $T E^{(p)}(t)$ ). Unlike Fig. 3.10(a), Fig. 3.10(b) exhibits localized high energy particles $p=145$ and $p=60$ (low mass particles), indicating localization of energy due to presence of disorder.

Eq. (3.37) is used to obtain Total Energy in wavenumber space, then Eq. (3.41) is used to bin the energy responses accordingly. The number of bins used for the computation here onwards is $B=32$ with a bandwidth $\Delta k=0.0982$. Fig. 3.11 shows the temporal evolution of total energy in wavenumber space for $\xi=0.35$ disordered chain (grayscale in the plot is $T E^{(k)}(t)$ ), $\mathscr{N}=85$ (Fig. 3.11 (a)) and 38 (3.11 (b)). A peak is initially observed at the initially agitated wavenumber $\left(k_{i n s}=\mathscr{N} \frac{2 \pi}{N+1}\right)$, the peak decreases as the time progresses, the decay rate is lower for lower wavenumber, which can be observed when Fig. 3.11 (a) and (b) are compared. Fig. 3.12 displays the binned total energy in binned wavenumber space for $T E^{(k)}(t)$ (from same data as in Fig. 3.11 (a)), Fig. 3.12 (b) represents the same total energy response averaged over 100 ensembles. Fig. 3.13 (a) shows the ensembled decay rate of the bin which contains 

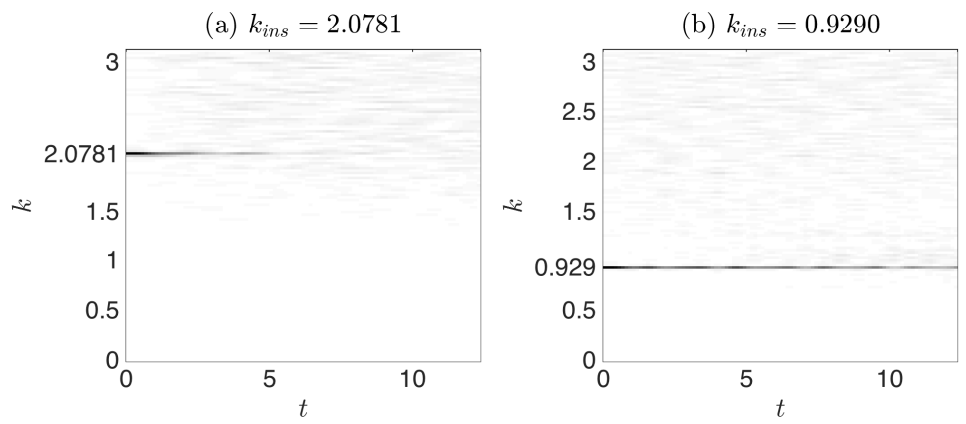

Figure 3.11: Total energy response $\left(T E^{(k)}(t)\right)$ in wavenumber space of a disordered granular chain $(\xi=0.35)$ initially agitated with (a) $k_{i n s}=2.0781(\mathscr{N}=85)$ and (b) $k_{\text {ins }}=0.9290(\mathscr{N}=38)$.

the initially agitated wavenumber $\left(22^{\text {nd }}\right.$ bin; $\left.k_{i n s}=2.0781\right)$, the energy from this bin is getting transferred to the other bins, the decay is well captured by the fit $\left\langle\hat{e}^{(r)}(t)\right\rangle=1-Q_{R R} t$ through Procedure 1 , where $Q_{R R}=0.0834$ and $\left\langle e^{(s)}(t)\right\rangle=$ $e^{-x_{1} t}+y_{1}$ through Procedure 2, where $x_{1}=0.3579$ and $y_{1}=0.3159$ (Sect. 3.3.2). Fig. 3.13 (b) displays the rise of energy in a bin which is receiving energy from the other bin, the rise is captured by the fit $\left\langle\hat{e}^{(s)}(t)\right\rangle=Q_{r s} t$ through Procedure 1 , where $Q_{r s}=0.0174$ and $\left\langle\hat{e}^{(r)}(t)\right\rangle=x_{2}\left(1-e^{-t / y_{2}}\right)$ through Procedure 2, where $x_{2}=0.1215$ and $y_{2}=2.3640$. Similar data for $18^{\text {th }}$ bin are shown in Fig. 3.14 (a) for $k_{i n s}=1.7114$, which is captured by the fit $\left\langle\hat{e}^{(r)}(t)=1-Q_{R R} t\left(Q_{R R}=0.0639\right)\right.$ through Procedure 1 and $\left\langle\hat{e}^{(s)}(t)\right\rangle=e^{-x_{1} t}+y_{1}\left(x_{1}=0.3488\right.$ and $\left.y_{1}=0.3970\right)$ for Procedure 2. The rise in the $22^{\text {nd }}$ bin is captured by $\left\langle\hat{e}^{(s)}(t)\right\rangle=Q_{r s} t$ through Procedure 1, where $Q_{r s}=0.0283$ and $\left\langle\hat{e}^{(r)}(t)\right\rangle=x_{2}\left(1-e^{-t / y_{2}}\right)$ through Procedure 2, where $x_{2}=0.1998$ and $y_{2}=2.5488$. It can be observed that the energy of the bin which is initially agitated decays and achieves a steady state. On the contrary, the energy of the bin, which is receiving energy, starts increasing and achieves a steady state.

Using the set of $k_{\text {ins }}$ (where $\mathscr{N}=2,6,10, \ldots, 126$, one mode from every bin, hence, encompassing all the 32 bins) and the fitting equations mentioned in Sect. 3.3.2, the components of the transfer matrix are computed for an ensemble of $100 \xi=0.35$ disordered granular chain. Fig. 3.15 displays the transfer matrix computed through Procedure 1. It can be observed that the matrix is antisymmetric, with higher negative values along the diagonal at higher bin 
(a) $\hat{e}^{(r)}(t), k_{\text {ins }}=2.0781$

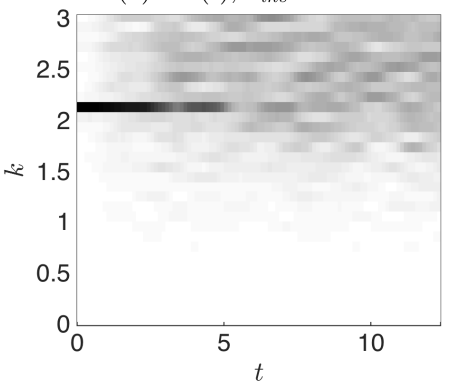

(b) $\left\langle\hat{e}^{(r)}(t)\right\rangle, k_{\text {ins }}=2.0781$

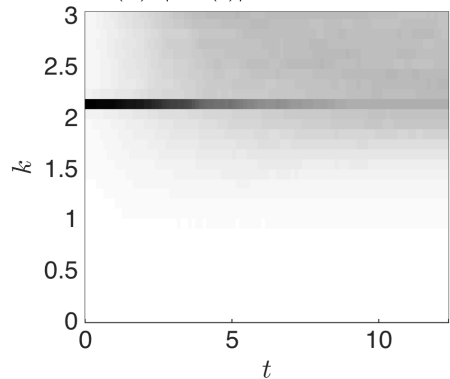

Figure 3.12: (a) Binned total energy response $\left(\hat{e}^{(22)}(t)\right)$ of Fig. 3.11 (a). (b) Binned total energy response averaged over 100 ensembles $\left(\left\langle\hat{e}^{(22)}(t)\right\rangle\right)$.

(a) $\left\langle\hat{e}^{(22)}(t)\right\rangle, k_{i n s}=2.0781$

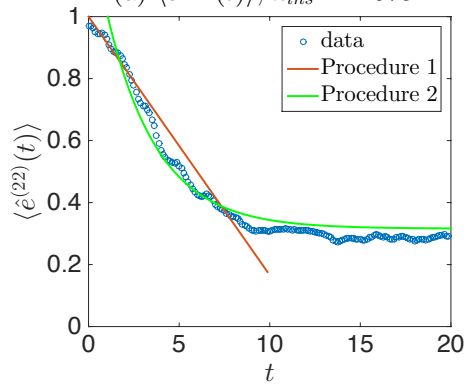

(b) $\left\langle\hat{e}^{(18)}(t)\right\rangle, k_{\text {ins }}=2.0781$

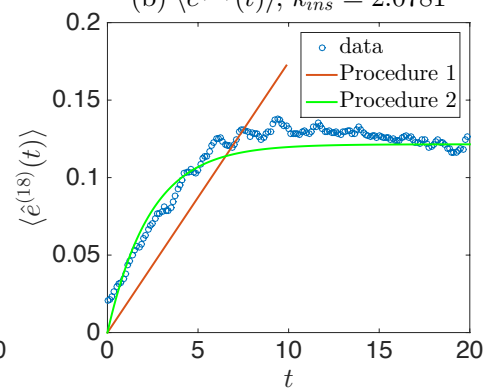

Figure 3.13: (a) Decaying binned total energy response of initially agitated bin 22 for $k_{\text {ins }}=2.0781$ with the fits following Procedure 1 and 2. (b) Increasing binned total energy response of a bin 18 receiving energy with the fits following Procedure 1 and 2. Only 80 initial time steps from the data have been used for Procedure 1 fit. 

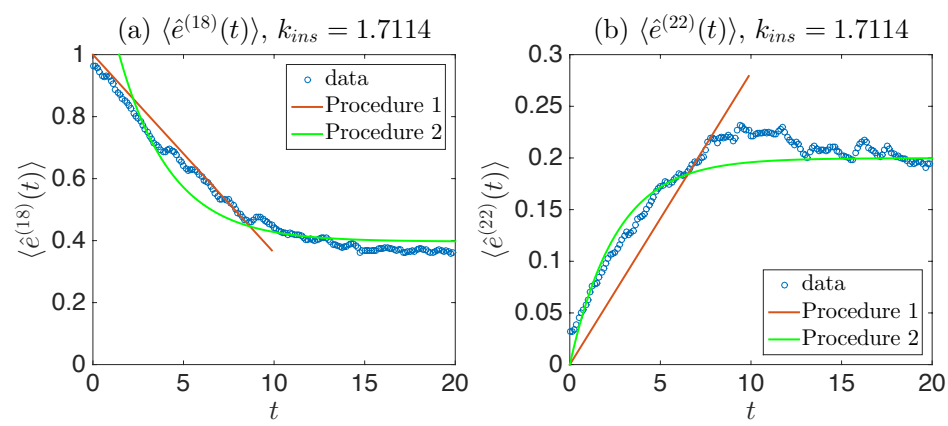

Figure 3.14: (a) Decaying binned total energy response of initially agitated bin 18 for $k_{i n s}=1.3990$ with the fits following Procedure 1 and 2. (b) Increasing binned total energy response of bin 22 receiving energy with the fits following Procedure 1 and 2. Only 80 initial time steps from the data have been used for Procedure 1 fit.

values (except the diagonal elements) and in the upper right corner of the matrix. Fig. 3.16 (a) represents the diagonal elements of the $\mathbf{Q}$ matrix computed from Procedure 1 (Fig. 3.15). The value $-Q_{R R}$ first rapidly increases (till $10^{\text {th }}$ bin), then decreases (till $27^{\text {th }}$ bin) and then slightly increases. However, the rapid decay can be attributed to the fact that lower bins/wavenumbers achieve a steady state fast as it can be observed from Fig. 3.16 (b), where $\left\langle\hat{e}^{(r)}(t)\right\rangle$ is plotted with time (averaged over 100 ensembles), leading to higher negative slopes and hence, $-Q_{R R}$. Fig. 3.17 (a) displays the $\mathbf{Q}$ computed by Procedure 2. This matrix is anti-symmetric as well. The non-diagonal elements look similar to the non-diagonal elements in Figure 3.15 except the upper right corner of the matrix has much higher values for lower wavenumber bins. Fig. 3.17 (b) represents the $-Q_{R R}$ elements of $\mathbf{Q}$ (Fig. 3.17 (a)), it shows that $-Q_{R R}$ increases and then saturates after some time; Besides featuring much larger values, it is not able to capture the features as shown in Fig. 3.17.

Reverse Modeling Frequency Propagation Using Eq. 3.38 and the $\mathbf{Q}$ matrix computed in Fig. 3.15, the frequency propagation of specific bins can be computed (the final purpose of the Master Equation formulation, here this computation can serve as cross-validation), which has been done for $22^{\text {nd }}$ bin in Fig. 3.18 (a) and $7^{\text {th }}$ bin in Fig. 3.18 (b). It is observed that the higher frequency 


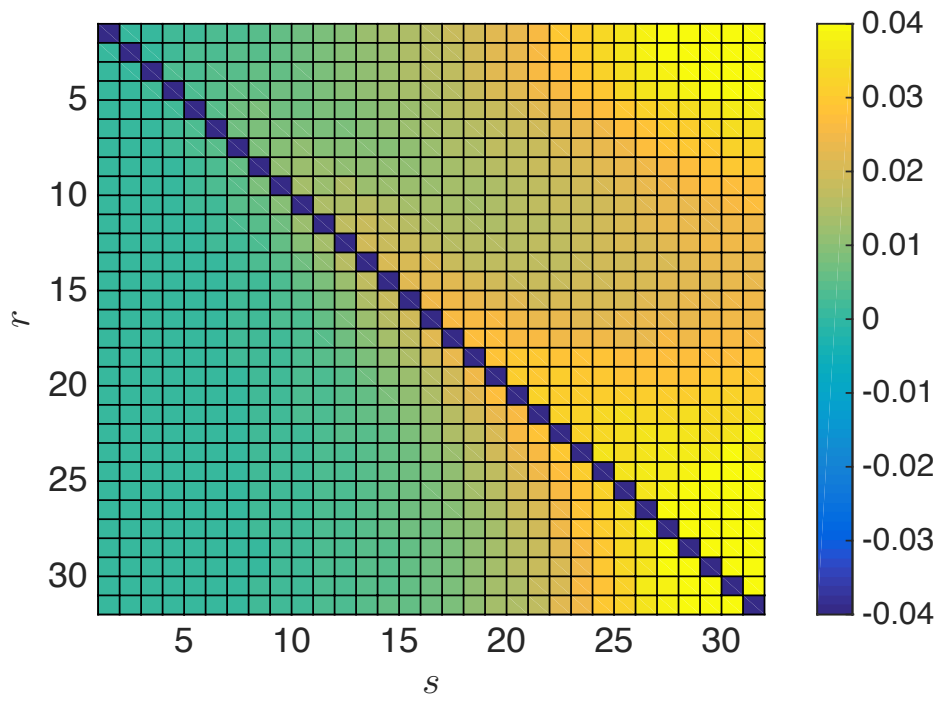

Figure 3.15: Components of $\mathbf{Q}$ matrix computed through Procedure 1 (Sect. 3.3.2). The diagonal elements $\left(Q_{R R}\right)$ are the attenuation coefficients of the total energy in band/bin $r$. The corresponding non-diagonal components $Q_{r s}$ are the increase rates of total energy in the bin $s$ after receiving energy from bin $r$.
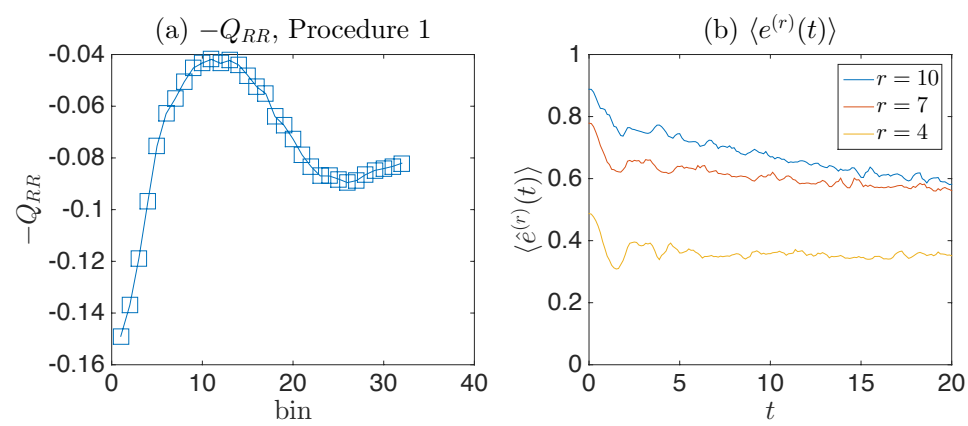

Figure 3.16: (a) Diagonal components of $\mathbf{Q}$ from Fig. 3.15 (Procedure 1) (b) Binned energy responses of low wavenumber bins achieving faster steady state. 

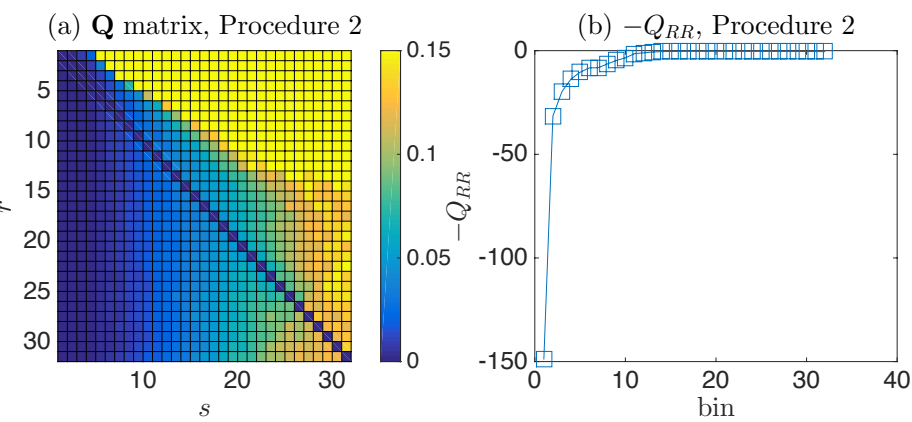

Figure 3.17: (a) $Q$ matrix obtained via Procedure 2 after assembling all the components $-Q_{R R}$ and $Q_{r s}$ (Sect. 3.3.2) (b) Diagonal components of $\mathbf{Q}$.

bin ( $22^{\text {nd }}$ bin) looses energy faster than the lower frequency bin ( $7^{\text {th }}$ bin) to other frequency bins/bands, it indicates that the lower frequency passes and the higher frequency attenuates, a fundamental frequency propagation characteristic in disordered granular media. The tuning of the parameters of $Q$ matrix is still in progress (Fig. 3.15 is not normalized). However, the frequency propagation characteristics is qualitatively captured by Fig. 3.18 but lacks a strong evidence for the localization close to the source.
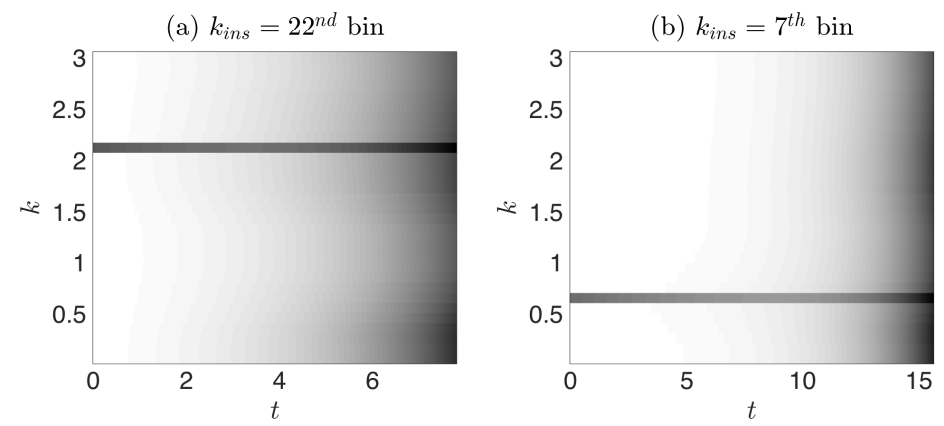

Figure 3.18: (a) Frequency propagation of $22^{\text {nd }}$ bin (b) $7^{\text {th }}$ bin computed using the $\mathbf{Q}$ matrix (Fig. 3.15). 


\subsection{Conclusion}

A mass disordered granular chain with linearized Hertzian repulsive interaction force between the granules is used as a model granular system (Sect. 3.2). The system is then used for studying the energy propagation characteristics having two types of initial conditions:

- Impulse initial condition for studying energy propagation with distance (Sect. 3.2.2).

- Sinusoidal standing wave initial condition for studying energy propagation across wavenumbers with time (Sect. 3.2.3)

Sect. 3.4.1 shows the existence of twin peaks when the total energy signal is plotted with distance from the source for disordered granular chains (Fig. 3.2); unlike ordered chains which have only one peak. The peak near the source is attributed to weak localization due to disorder, whereas, the second peak is the propagating coherent wavefront. The weak localization decay curve is observed to observed to be invariant with time and exhibits a power law like relationship (Fig. 3.4) with distance from the source, where the rate of decay increases with increasing disorder parameter $(\xi)$. However, the second peak (wavefront) rapidly decreases with distance from the source and more strongly with increasing $\xi$. Eq. 3.34 (center of energy; Fig. 3.6) and Eq. 3.35 (mean squared width of energy; Fig. 3.7) clearly show diffusive-like propagation of energy. The center of energy's propagation speed decreases with $\xi$. Disorder leads to confinement of center of energy within a finite space which decreases with increasing $\xi$. Fig. 3.6 displays ballistic propagation of energy for an ordered chain, which becomes slightly superballistic for small disorder for small disorder and then superdiffusive, diffusive and subdiffusive with increase in disorder $\xi$, successively.

Sect. 3.4.2 focuses on the total energy evolution of the standing wave sinusoidal initial condition, as is used for studying the transfer of energy across wavenumbers in disordered chains. The energy becomes localized, here, around lower masses in the chain. Fig. 3.11 shows that the energy in higher wavenumbers decays faster than the energy in lower wavenumbers. Two procedures were introduced in Sect. 3.3.2 for computing the components of transfer matrix $\mathbf{Q}$ in the Master Equation. Fig. 3.15 and Fig. 3.17 (a) show two respectively computed transfer matrices. These matrices can be used in the Master Equation for modeling a $\xi=0.35$ disordered granular chain easing the computational expense 
relative to the full model with all degrees of freedom, but still the characteristics of energy transfer across the wavenumbers.

The Weak Localization effect and the diffusion model are interesting features of disordered media, which can be modeled and parametrized to understand and predict the material properties. The Master Equation acts as a stochastic model for modeling wave propagation in disordered media, the transfer matrix can be improved with better fitting curves and better formulation, which may also incorporate the nonlinear interactions between different wavenumber bands. There are some open issues in fit procedure, the initial time interval to be used for fitting the decay of the agitated bin or the rise in energy of the nonagitated bin, one option would be to select the time interval according to the initially inserted wavenumber through dispersion relation. As mentioned previously in Sect. 3.1, the computed Master Equation can be used for continuum analyses associated with larger systems, which contains information about the micro-structure in the form of components of the transfer matrix and needs to be calibrated and validate experimentally. 


\title{
Chapter 4
}

\section{Rotational Sound Propagation in Disordered Frictional Disks}

\begin{abstract}
Where a body is in motion, there exists space and time, the simplest sentient creature in this world would thus be a measure of them. Our hearing, and perhaps our seeing too, consists of a counting of oscillations.
\end{abstract}

Georg Christoph Lichtenberg

We employ numerical simulations to understand the evolution of elastic standing waves in disordered frictional disk systems, where the dispersion relations of rotational sound modes are analyzed in detail. As in the case of frictional particles on a lattice, the rotational modes exhibit an "optical-like" branch in the dispersion relation in the high frequency regime representing a shoulder of the vibrational density of states and fast oscillations of the autocorrelations of rotational velocities. A lattice based model describes the dispersion relations of the rotational modes for small wavenumbers. The rotational modes are perfectly explained by the model if tangential elastic forces between the disks in contact are large enough. If the tangential forces are comparable with or smaller than normal forces, the model fails for short wavelengths. However, the dispersion relation of the rotational modes then follows the model prediction for transverse modes, implying that the fast oscillations of disks' rotations switch to acoustic sound behavior. We observe such a transition of the rotational modes by eigenvectors of disordered frictional disks to identify upper and lower limits of the frequency-bands. Those are not reversed over 
the whole range of tangential stiffness, which is a remarkable difference from the rotational sound in frictional particles on lattice. ${ }^{1}$

\subsection{Introduction}

Granular material exists at all spatial scales in nature, whether it is soil or space dust. An important feature of such media is its momentum and energy transport characteristics, either for oil/gas exploration, geotechnical investigations of soil, or understanding of seismic waves and earthquakes [166]. Sound waves in granular material are also useful to determine its mechanical properties but to better interpret measurements a model which incorporates the microstructure is required [175]. In addition, tangential forces (and friction) between grains in contacts are intrinsic to granular material, which induces micropolar rotations of the constituents. Incorporating the rotational degrees of freedom for the internal microstructure of granular material has been the basis for Cosserat continuum theory [39]. So far, one of the most striking aspects of Cosserat behavior (micropolar rotations of constituent grains) is the existence of rotational sound [170]. This has been extensively studied by experiments [121] and numerical simulations [119] of frictional granular crystals, where the characteristic dispersion relations are well predicted by the theory of frictional particles on lattice [120].

Despite these successes of continuum theory, spatial configurations of the constituent grains in nature are mostly disordered. Recently, various anomalies in acoustic sound in disordered media have been clarified by experiments on amorphous solids $[16,17]$ and numerical simulations of randomly arranged particles [60, 116, 124, 127], where small dips in phase speeds and deviations from the theory of Rayleigh scattering are commonly observed. Anomalies in the vibrational density of states (vDOS), e.g. the Boson peak near the glass transition temperature $[66,105,123,126,168]$ and a characteristic plateau near the jamming transition $[180,181,219,220]$, are not predicted by the classical theory of elasticity. Moreover, shock waves $[63,64,136,205]$ and solitary wave propagation [197, 207] are interesting properties of real disordered systems though they are due to nonlinearity of the interaction forces and anharmonic vibrations. Neglecting the latter phenomena and reducing on a rather simple 2D model system, it is feasible to focus on the question: How does configurational disorder alter the dispersion relations of rotational sound?

\footnotetext{
${ }^{1}$ (To be) submitted as: Saitoh K., Shrivastava, R.K. \& Luding, S. (2018). Rotational sound in disordered granular materials.
} 
In this chapter, we investigate sound in disordered frictional disks by numerical simulations. Introducing the dynamical matrix, we analyze effects of tangential forces on the vDOS and demonstrate the evolution of purely elastic standing waves of Longitudinal (L), transverse (TR) and rotational (RT) modes. We calculate autocorrelational functions and spectra of L, TR and RT velocities to find the dispersionn relations. Then, we quantify the dependance of the dispersion relations on the strength of the tangential forces, where we introduce a modified lattice-based model to describe the "optical-like" branch of the rotational sound modes. We show that the RT mode switches from optical-like to acoustic branches at characteristic wavelengths, which monotonously increase with decreasing the strength of tangential forces. We examine these transitions by analysing the eigenvectors of the disk-system and also discuss the frequency bands of the RT mode.

\subsection{Numerical Method}

To study sound in disordered frictional disks, we introduce their dynamical matrix. If the system consisting of $N$ disks is initially in mechanical equilibrium, small vibrations of the disks around initial positions, $\left\{\mathbf{r}_{i}(0)\right\}(i=1, \ldots, N)$, are described by the equations of motion,

$$
\mathscr{M} \ddot{\mathbf{u}}(t)=-\mathscr{D} \mathbf{u}(t),
$$

where the $3 N$-dimensional displacement vector,

$\mathbf{u}(t) \equiv\left(\mathbf{u}_{1}(t), \theta_{1}(t), \ldots, \mathbf{u}_{N}(t), \theta_{N}(t)\right)^{\mathrm{T}}$ with time $t$, includes not only translational displacements, $\mathbf{u}_{i}(t) \equiv \mathbf{r}_{i}(t)-\mathbf{r}_{i}(0)$, but also angular displacements, $\theta_{i}(t)$. On the left-hand-side of Eq. (4.1), the $3 N \times 3 N$ mass matrix, $\mathcal{M}$, is diagonal with the diagonal elements, i.e. $m_{i}, m_{i}, I_{i}$ in sequence, where $m_{i}$ and $I_{i}=m_{i} d_{i}^{2} / 8$ with diameter of the disk $i, d_{i}$, are the mass and moment of inertia, respectively. On the other hand, $\mathscr{D}$ is the $3 N \times 3 N$ dynamical matrix which consists of second derivatives of elastic energy, $E=\sum_{i>j} e_{i j}$. The pairwise potential, $e_{i j}$, is decomposed into potential energies stored in normal and tangential directions, i.e. $e_{i j}=\left(k_{n} \xi_{i j}^{2} / 2\right)+\left(k_{t} \mathbf{u}_{i j}^{\perp 2} / 2\right)$ for $\xi_{i j}>0$ and $e_{i j}=0$ otherwise, where $\xi_{i j}$ represents the overlap between the disks, $i$ and $j$, and $k_{n}$ and $k_{t}$ are normal and tangential stiffness, respectively. Here, $\mathbf{u}_{i j}^{\perp} \equiv \mathbf{u}_{i j}-\mathbf{u}_{i j}^{\|}-\boldsymbol{\theta}_{i j} \times \mathbf{n}_{i j}$ is the relative displacement in tangential direction, where we introduced relative displacements as $\mathbf{u}_{i j} \equiv \mathbf{u}_{i}-\mathbf{u}_{j}, \mathbf{u}_{i j}^{\|} \equiv\left(\mathbf{u}_{i j} \cdot \mathbf{n}_{i j}\right) \mathbf{n}_{i j}$, and $\boldsymbol{\theta}_{i j} \equiv\left(d_{i} \theta_{i}+d_{j} \theta_{j}\right) \mathbf{n}_{z} / 2$ with unit vectors, $\mathbf{n}_{i j} \equiv\left(\mathbf{r}_{i}-\mathbf{r}_{j}\right) /\left|\mathbf{r}_{i}-\mathbf{r}_{j}\right|$ and $\mathbf{n}_{z}$ (which is parallel to the $z$-axis). The dynamical 
matrix is given by

$$
\mathscr{D}=\left(\begin{array}{ccc}
\frac{\partial^{2} E}{\partial x_{i} \partial x_{j}} & \frac{\partial^{2} E}{\partial x_{i} \partial y_{j}} & \frac{\partial^{2} E}{\partial x_{i} \partial \theta_{j}} \\
\frac{\partial^{2} E}{\partial y_{i} \partial x_{j}} & \frac{\partial^{2} E}{\partial y_{i} \partial y_{j}} & \frac{\partial^{2} E}{\partial y_{i} \partial \theta_{j}} \\
\frac{\partial^{2} E}{\partial \theta_{i} \partial x_{j}} & \frac{\partial^{2} E}{\partial \theta_{i} \partial y_{j}} & \frac{\partial^{2} E}{\partial \theta_{i} \partial \theta_{j}}
\end{array}\right)
$$

To generate initial disordered configurations, $\left\{\mathbf{r}_{i}(0)\right\}$, we use MD simulations of frictionless disks (i.e. $k_{t}=0$ ). In order to avoid crystallization, we randomly distribute a 50:50 binary mixture of the $N$ disks in a periodic box, where different kinds of disks have the same mass, $m$, and different diameters, $d_{L}$ and $d_{S}$ (with their ratio $d_{L} / d_{S}=1.4$ ). Then, we minimize elastic energy, $E_{n}=\sum_{i>j} k_{n} \xi_{i j}^{2} / 2$, with the aid of FIRE algorithm [24]. We stop the energy minimization once the maximum of disk acceleration becomes less than $10^{-6} k_{n} d_{0} / \mathrm{m}$, where $d_{0} \equiv\left(d_{L}+d_{S}\right) / 2$ is the mean disk diameter. During the energy minimization, the potential energy is not stored in tangential direction so that the system is still in mechanical equilibrium even if we switch on the tangential force (i.e. $E=E_{n}$ though $k_{t}>0$ ). Therefore, our disks are initially unstressed in tangential direction ${ }^{2}$.

\subsection{Numerical Results}

First, we investigate normal modes of disordered frictional disks. Assuming vibrational motions of the disks around initial positions, we substitute $\mathbf{u}(t)=\overline{\mathbf{u}} e^{I \omega t}$ to Eq. (4.1), where $\overline{\mathbf{u}}, I$, and $\omega$ are the amplitude, imaginary unit, and frequency, respectively. Then, we numerically solve an eigenvalue problem, $\mathscr{M}^{-1} \mathscr{D} \overline{\mathbf{u}}=\lambda_{q} \overline{\mathbf{u}}$, to find eigen frequencies given by square roots of the eigenvalues, i.e. $\omega_{q} \equiv \sqrt{\lambda_{q}}$ $(q=1, \ldots, 3 N)^{3}$. Figure 4.1 displays distributions of the eigen frequencies, or $v i$ brational density of states (vDOS), of disordered frictional disks (the solid lines), where we averaged each vDOS over 100 different initial positions of $N=2048$ disks. In this figure, we change the stiffness ratio, $\rho \equiv k_{t} / k_{n}$, and also plot our result of frictionless disks, $\rho=0$ (the dotted line). As can be seen, the highest

\footnotetext{
${ }^{2}$ The stressed systems can be made if we prepare initial disordered configurations with tangential forces $\left(k_{t}>0\right)$. However, tangential forces make the system history-dependent and we postpone such the case to future work. See also [122] for the difference between stressed and unstressed systems.

${ }^{3}$ The eigenvalue, $\lambda_{q}$, is a (real) positive number so that the eigen frequency, $\omega_{q}=\sqrt{\lambda_{q}}$, is also real positive.
} 
peak around $\omega t_{0} \simeq 2.3$ for frictionless disks shifts to higher frequencies with the increase of stiffness ratio, where the time unit is given by $t_{0} \equiv \sqrt{m / k_{n}}$. In addition, a shoulder, which does not exist in the vDOS of frictionless disks, develops in a high frequency regime $\left(2.3 \lesssim \omega t_{0} \lesssim 6.6\right)$ so that the cutoff frequency, $\omega_{c}$, at the end of vDOS, i.e. $g(\omega)=0$ if $\omega \geq \omega_{c}$ (as indicated by the arrow in Fig. 4.1), greatly increases from $2.3 t_{0}^{-1}$ to $6.6 t_{0}^{-1}$. Therefore, the high frequency-band the shaded region in Fig. 4.1) is characteristic of disordered frictional disks. Note that there is a second peak if the stiffness ratio is small (as indicated by the vertical arrow in Fig. 4.1). Moreover, the vDOS in the high frequency regime is insensitive to the area fraction which we fix to $\phi=0.9$ in the following analyses.

Next, we study how elastic waves actually propagate through disordered frictional disks. Employing a similar method with Gelin et al. [60], we nu-

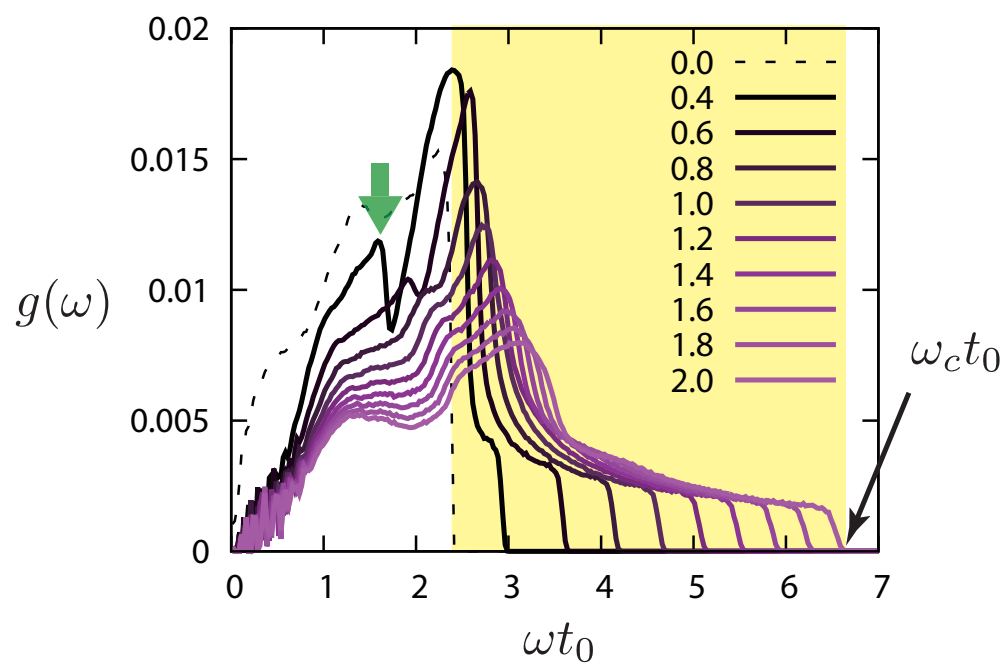

Figure 4.1: (Color online) The vDOS of disordered frictional disks (the solid lines), where the area fraction is fixed to $\phi=0.9$ and the stiffness ratio, $\rho \equiv k_{t} / k_{n}$, increases as listed in the legend. The dotted line is the vDOS of disordered frictionless disks, i.e. $\rho=0$, with the same area fraction. The shaded region represents a high frequency-band, where the vDOS ends at cutoff frequency, $\omega_{c}$, as indicated by the arrow. The green vertical arrow indicates the second peak of vDOS for $\rho=0.4$. 
Table 4.1: Three different elastic waves, L, TR, and RT modes, excited by different combinations of amplitudes, $\mathbf{A}=\left(A_{x}, A_{y}\right)$ and $A_{\theta}$, and wave number vector, $\mathbf{k}=\left(k_{x}, k_{y}\right)$, where $k \equiv|\mathbf{k}|$. The values of $A_{x}$ and $A_{y}$ are given in the unit, $d_{0} / t_{0}$, while $A_{\theta}$ is listed in the unit, $t_{0}^{-1}$.

\begin{tabular}{cccccc} 
& $A_{x}$ & $A_{y}$ & $A_{\theta}$ & $k_{x}$ & $k_{y}$ \\
\hline $\mathrm{L}$ & $10^{-3}$ & 0 & 0 & $k$ & 0 \\
& 0 & $10^{-3}$ & 0 & 0 & $k$ \\
$\mathrm{TR}$ & $10^{-3}$ & 0 & 0 & 0 & $k$ \\
& 0 & $10^{-3}$ & 0 & $k$ & 0 \\
$\mathrm{RT}$ & 0 & 0 & $10^{-3}$ & $k$ & 0 \\
& 0 & 0 & $10^{-3}$ & 0 & $k$
\end{tabular}

merically integrate the equations of motion, Eq. (4.1), under periodic boundary conditions, where the number of disks is now increased to $N=32768$. Here, initial velocities of the disks are given by sinusoidal standing waves, i.e. $\left\{\dot{\mathbf{u}}_{i}(0), \dot{\theta}_{i}(0)\right\}=\left\{\mathbf{A}, A_{\theta}\right\} \sin \left(\mathbf{k} \cdot \mathbf{r}_{i}(0)\right)(i=1, \ldots, N)$, satisfying the periodic boundary conditions, where $\mathbf{A}$ and $A_{\theta}$ are small amplitudes and $\mathbf{k}$ is the wave number vector. Combining different amplitudes and wave number vector, we can demonstrate propagation of three different elastic waves, i.e. longitudinal (L), transverse (TR), and rotational (RT) modes, as listed in Table $4.1^{4}$. Note that the RT mode represents the propagation of disks' rotations [119] and does not exist in frictionless disks. Because Eq. (4.1) describes purely harmonic oscillations of the disks around initial positions, any anharmonic behavior, e.g. due to opening and closing contacts and changes of the associated contact probability density [162], is not taken into account.

From numerical solutions of Eq. (4.1), i.e. $\left\{\dot{\mathbf{u}}_{i}(t), \dot{\theta}_{i}(t)\right\}$, we analyze the time development of vibrational motions. Introducing Fourier transforms of the numerical solutions as $\left\{\dot{\mathbf{u}}_{\mathbf{k}}(t), \dot{\theta}_{\mathbf{k}}(t)\right\}=\sum_{i=1}^{N}\left\{\dot{\mathbf{u}}_{i}(t), \dot{\theta}_{i}(t)\right\} e^{-I \mathbf{k} \cdot \mathbf{r}_{i}(t)} 5$, we define longitudinal and transverse velocities as $\dot{\mathbf{u}}_{\mathbf{k}}^{\|}(t) \equiv\left\{\dot{\mathbf{u}}_{\mathbf{k}}(t) \cdot \hat{\mathbf{k}}\right\} \hat{\mathbf{k}}$ and $\dot{\mathbf{u}}_{\mathbf{k}}^{\perp}(t) \equiv \dot{\mathbf{u}}_{\mathbf{k}}(t)-\dot{\mathbf{u}}_{\mathbf{k}}^{\|}(t)$, respectively, where $\hat{\mathbf{k}} \equiv \mathbf{k} / k$ with $k \equiv|\mathbf{k}|$ is a unit vector parallel to the wave number vector. Then, we calculate normalized velocity autocorrelation functions (VAFs) of longitudinal, transverse, and rotational velocities as $C_{l}(k, t)=$ $\left\langle\dot{\mathbf{u}}_{\mathbf{k}}^{\|}(t) \cdot \dot{\mathbf{u}}_{-\mathbf{k}}^{\|}(0)\right\rangle /\left\langle\left|\dot{\mathbf{u}}_{\mathbf{k}}^{\|}(0)\right|^{2}\right\rangle, C_{t}(k, t)=\left\langle\dot{\mathbf{u}}_{\mathbf{k}}^{\perp}(t) \cdot \dot{\mathbf{u}}_{-\mathbf{k}}^{\perp}(0)\right\rangle /\left\langle\left|\dot{\mathbf{u}}_{\mathbf{k}}^{\perp}(0)\right|^{2}\right\rangle$,

\footnotetext{
${ }^{4}$ Due to the tangential force and interlocking of the disks, every mode is excited by any combinations of amplitudes and wave number vector. For example, the combination in the first line of Table 4.1 excites not only L, but also TR and RT though their intensities are quite weak.

${ }^{5}$ The position, $\mathbf{r}_{i}(t)$, is also obtained from numerical integrations of Eq. (4.1).
} 
and $C_{r}(k, t)=\left\langle\dot{\theta}_{\mathbf{k}}(t) \dot{\theta}_{-\mathbf{k}}(0)\right\rangle /\left\langle\left|\dot{\theta}_{\mathbf{k}}(0)\right|^{2}\right\rangle$, respectively. Figure 4.2 shows the time development of the VAFs, $C_{\alpha}(k, t)(\alpha=l, t, r)$ (the open circles), where we also plot our results of frictionless disks (the dotted lines in (a) and (b)). As expected, in both frictional and frictionless disks, the oscillations of L mode are faster than those of TR mode (Figs. 4.2(a) and (b)). In addition, the oscillations of $\mathrm{L}$ and TR modes become faster in frictional disks, implying that the tangential forces increase macroscopic elastic constants. We also note that the decay of their VAFs, which is caused by scattering of L and TR modes ${ }^{6}$, becomes slower in frictional disks. On the other hand, the oscillation of RT mode is very much faster than those of L and TR modes (Fig. 4.2(c)), indicating that normal modes in the high frequency-band (Fig. 4.1) are closely related to rotations of frictional disks. Moreover, the VAF of RT mode decays mush faster than those of L and TR modes, implying strong scattering of RT mode, which is not reported previously [119].

To further investigate propagation of elastic waves, we calculate power spectra of longitudinal, transverse, and rotational velocities as $S_{l}(k, \omega)=\left\langle\left|\tilde{\mathbf{u}}_{\mathbf{k}}^{\|}(\omega)\right|^{2}\right\rangle$, $S_{t}(k, \omega)=\left\langle\left|\tilde{\dot{\mathbf{u}}}_{\mathbf{k}}^{\perp}(\omega)\right|^{2}\right\rangle$, and $S_{r}(k, \omega)=\left\langle\left|\tilde{\theta}_{\mathbf{k}}(\omega)\right|^{2}\right\rangle$, respectively, where Fourier trans-

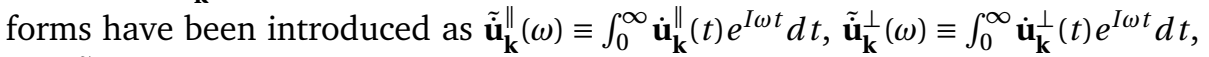
and $\tilde{\theta}_{\mathbf{k}}(\omega) \equiv \int_{0}^{\infty} \dot{\theta}_{\mathbf{k}}(t) e^{I \omega t} d t$. Figure 4.3 displays three dimensional plots of logarithms of the power spectra, i.e. $\log S_{\alpha}(k, \omega)(\alpha=l, t, r)$, which show dispersion relations of the elastic waves. In the dispersion relations of $\mathrm{L}$ and $\mathrm{TR}$ modes (Figs. 4.3(a) and (b)), we observe ordinary acoustic branches, where the speed defined as the slope, $\lim _{k \rightarrow 0} \omega / k$, of L mode is larger than that of TR mode. On the other hand, the RT mode exhibits an "optical-like" branch (Fig. 4.3(c)) as previously found in frictional granular crystals [119]. The optical-like branch exists only in a high frequency regime $\left(2.5 \leq \omega t_{0} \leq 4.7\right)$ as indicated by the double-headed vertical arrow in Fig. 4.3(c). Because the vDOS is given by an integral of dispersion relation over the whole wave numbers [175], this regime corresponds to the high frequency-band in the vDOS (Fig. 4.1) and represents the fast oscillation of the VAF of RT mode (Fig. 4.2(c)). In addition, an opticallike branch also exists in Fig. 4.3(b), while an acoustic branch is also found in Fig. 4.3(c). Though their intensities are quite weak, such the coexistence of two branches means that rotations are strongly coupled with transverse motions [119].

\footnotetext{
${ }^{6}$ Because our system conserves total energy, the decay of VAFs is not caused by energy dissipation.
} 

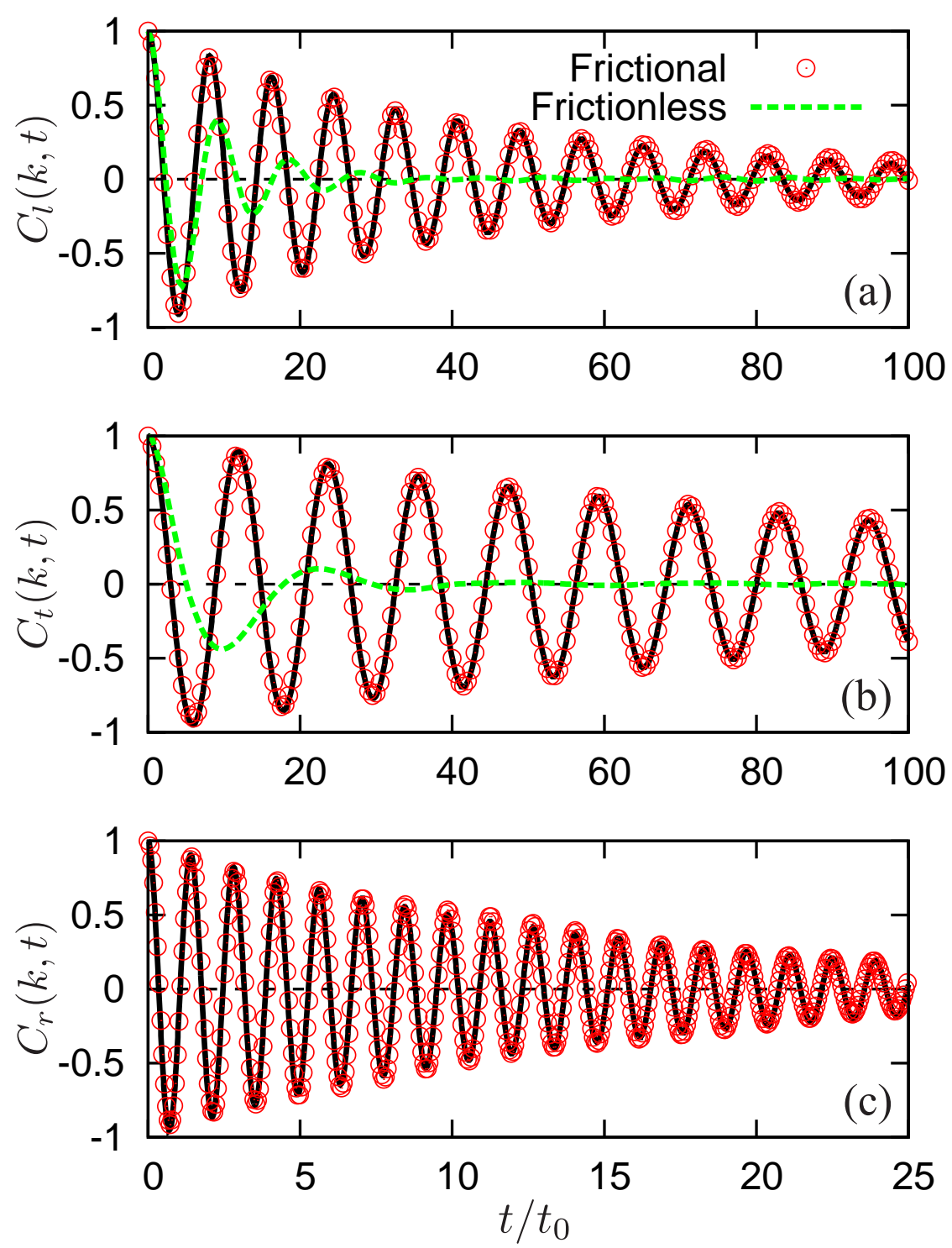

Figure 4.2: (Color online) The VAFs of (a) longitudinal, (b) transverse, and (c) rotational velocities, where we used the parameters, $\phi=0.9$ and $\rho=1.0$, and fixed the wave number to $k d_{0} \simeq 0.38 \pi$. The open circles are obtained from numerical solutions of Eq. (4.1) and the solid lines represent the damped oscillations, Eq. (4.3). In (a) and (b), our results of frictionless disks $(\rho=0)$ with the same area fraction are also shown (the dotted lines). Note the different horizontal scale in (c). 

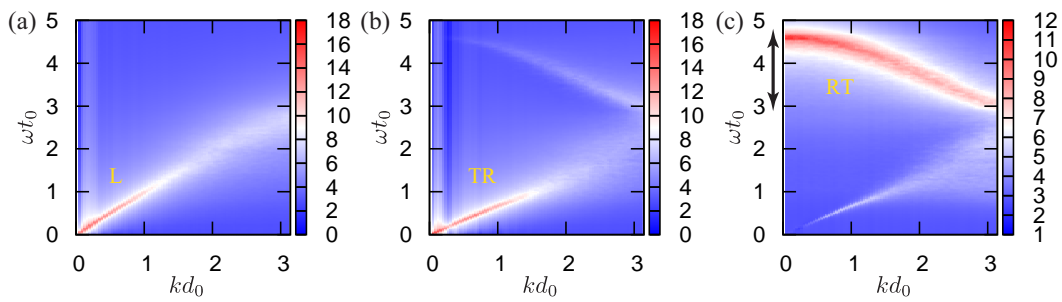

Figure 4.3: (Color online) Dispersion relations of (a) the longitudinal (L), (b) transverse (TR), and (c) rotational (RT) modes, where the area fraction and stiffness ratio are given by $\phi=0.9$ and $\rho=1.0$, respectively. The gray scale represents $\log$ arithms of power spectra, i.e. (a) $\log S_{l}(\omega, k)$, (b) $\log S_{t}(\omega, k)$, and (c) $\log S_{r}(\omega, k)$, where the frequency and wave number are scaled by the units, $t_{0}$ and $d_{0}$, respectively. The frequency-band of RT mode is indicated by the double-headed vertical arrow in (c).

\subsection{Theoretical Model}

The optical-like branch of RT mode, which is spanning the high frequency-band, is a striking feature of disordered frictional disks. Thus, to quantitatively analyze its sound properties, we extract dispersion relations from numerical solutions of Eq. (4.1). For this purpose, we fit damped oscillations to the VAFs as

$$
C_{\alpha}(k, t)=e^{-\gamma_{\alpha}(k) t} \cos \omega_{\alpha}(k) t
$$

( $\alpha=l, t, r$ ), where the dispersion relation of each mode is given by $\omega_{\alpha}(k)$ and the scattering of each mode is quantified by the attenuation coefficient, $\gamma_{\alpha}(k)^{7}$. The solid lines in Fig. 4.2 represent the damped oscillations, Eq. (4.3), where we confirm perfect agreements with the VAFs by adjusting the parameters, $\omega_{\alpha}(k)$ and $\gamma_{\alpha}(k)$. Small dips are observed in the phase speeds, $c_{\alpha}(k) \equiv \omega_{\alpha}(k) / k[60]$, while the attenuation coefficients obey the Rayleigh scattering, i.e. $\gamma_{\alpha}(k) \sim k^{3}$

\footnotetext{
${ }^{7}$ The Fourier transform of the damped oscillation, Eq. (4.3), is given by the Lorentzian, $S_{\alpha}(k, \omega)=$ $\gamma_{\alpha}(k) /\left[\left\{\omega-\omega_{\alpha}(k)\right\}^{2}+\gamma_{\alpha}(k)^{2}\right]$, which also well describes the power spectrum of $\alpha$ mode (Fig. 4.3) except for the weak intensities of optical-like and acoustic branches in Figs. 4.3(b) and (c), respectively.
} 
( $\alpha=l, t$ ), which is not the case in frictionless disks [60]. Figure 4.4 displays dispersion relations of optical-like RT mode, $\omega_{r}(k)$ (the symbols). As can be seen, the frequency-band shifts to higher frequencies with the increase of stiffness ratio, $\rho$ (as indicated by the arrow). To explain such a dependence of opticallike branches on $\rho$, we modify the discrete model of frictional grains on lattice [119], where the dispersion relations of TR and RT modes are given by similar forms,

$$
\begin{aligned}
& \omega_{t}(k)=t_{0}^{-1} c_{t} \sqrt{f(k, \rho)-\sqrt{g(k, \rho)}}, \\
& \omega_{r}(k)=t_{0}^{-1} c_{r} \sqrt{f(k, \rho)+\sqrt{g(k, \rho)}},
\end{aligned}
$$

respectively. In Eqs. (4.4) and (4.5), the prefactor, $c_{\alpha} \leq 1(\alpha=t, r)$, represents the decrease of sound speed by disorder and the functions are defined as

$$
\begin{aligned}
f(k, \rho) & =2 \sin ^{2}(k l)+9 \rho \cos ^{2}(k l)+11 \rho, \\
g(k, \rho) & =4 \sin ^{4}(k l)+\rho(300 \rho-4) \cos ^{2}(k l) \\
& -\rho\left(\frac{121}{4} \rho+21\right) \sin ^{2}(2 k l)+\rho(\rho+4) .
\end{aligned}
$$

In Eqs. (4.6) and (4.7), the length scale, $l$, is estimated by considering the first Brillouin zone, $|k l| \leq \pi / 2$ : Equating the maximum wave number in the model with $\pi / d_{0}$, we obtain $k_{\max }=\pi / 2 l \approx \pi / d_{0}$, i.e. $l \approx d_{0} / 2$. The lines in Fig. 4.4 are theoretical predictions of $\omega_{r}(k)$ (Eq. (4.5)), where we used $c_{r} \simeq 0.73$ and $l \simeq$ $0.446 d_{0}$. In this figure, all the dispersion relations are consistent with the lattice model in the long wave lengths, $k \lesssim \pi / 2 d_{0}$, as the difference in microstructures, i.e. order and disorder, should not affect the long wave properties. In addition, if the stiffness ratio is fairly large $(\rho<1.0)$, the dispersion relations are perfectly described by the model. However, if the stiffness ratio is small $(\rho>1.0)$, we observe deviations from the model in the short wave lengths, $k>k_{c}(\rho)$, where the characteristic wave number, $k_{c}(\rho)$, seems to monotonously decrease with the decrease of stiffness ratio.

To clarify the deviations in the short wave lengths, we carefully look at the dispersion relations with small stiffness ratios. Figure 4.5 shows (a) the logarithm of power spectrum, $S_{r}(k, \omega)$, and (b) dispersion relations, $\omega_{t}(k)$ and $\omega_{r}(k)$, obtained from the fitting to VAFs, Eq. (4.3), where the stiffness ratio is given by $\rho=0.2$. As can be seen in Fig. 4.5(a), the optical-like branch ends and drops to another branch at the characteristic wave number, $k_{c}(\rho)$, which makes a small 
gap in the frequency between $1.2<\omega t_{0}<2.5$. Accordingly, in Fig. 4.5(b), the dispersion relation of RT mode (the open squares) suddenly drops to lower values, while that of TR mode (the pluses) jumps at $k_{c}(\rho)$, such that the RT (TR) mode dominates lower (higher) frequencies in the short wave lengths, $k>k_{c}(\rho)$. The dispersion relation of RT mode deviates from the model of $\omega_{r}(k)$, Eq. (4.5) (the solid line), around $k_{c}(\rho)$. However, it is then well explained by the model of $\omega_{t}(k)$, Eq. (4.4) (the dotted line), after dropping to lower values in $k>k_{c}(\rho)$. Therefore, it seems that disks' rotations exhibit a transition from the opticallike fast oscillations to the acoustic wave behavior if the stiffness ratio is small $(\rho<1)$.

We further examine the transition by eigen vectors of dynamical matrix, $\overline{\mathbf{u}}_{q}$, i.e. displacements associated with each eigen frequency. Here, we quantify kinetic energy for each degree of freedom by $K_{v} \equiv \sum_{i} m \dot{\bar{u}}_{i v}^{2} / 2 N(v=x, y)$ and $Q \equiv \sum_{i} I_{i} \dot{\bar{\theta}}_{i}^{2} / 2 N$, where $\dot{\overline{\mathbf{u}}}_{q} \equiv \overline{\mathbf{u}}_{q} / t_{0}$ is introduced as an analog of velocities. Then, the translational energy, $K \equiv\left(K_{x}+K_{y}\right) / 2$, and rotational one, $Q$, represent the intensity of the $\mathrm{L}$ and TR modes and that of the RT mode, respectively. As shown in Fig. 4.6(a), if the stiffness ratio is not small, $K$ and $Q$ dominate low and high eigen frequencies, respectively. In this case, the acoustic L and TR branches in low frequencies are well separated from the optical-like RT branch in high frequencies (as in Fig. 4.3). In addition, it is clear that the shoulder of the vDOS (the dashed line in Fig. 4.6(a)) in high frequencies, $3<\omega t_{0}<4.6$, is owned by disks' rotations, $Q$. However, if the stiffness ratio is small (Fig. 4.6(b)), there are four regions, i.e. $K>Q$ in (i) and (iii) and $K<Q$ in (ii) and (iv), corresponding to the branches (i)-(iv) in the dispersion relations (Fig. 4.5(b)). Therefore, the transition from the optical-like to acoustic behavior of disks' rotations is also evidenced by the eigen vectors. Because the vDOS is given by integrating dispersion relations over the whole wave number range, its second peak (indicated by the vertical arrow in Fig. 4.6(b)) represents (ii) the RT mode on the acoustic branch.

The theoretical model also predicts frequency-bands of rotational mode. From Eq. (4.5), the frequency-bands are given by $\omega_{r}\left(k_{\max }\right) \leq \omega \leq \omega_{r}(0)$ if $\rho>1 / 7$ and $\omega_{r}(0) \leq \omega \leq \omega_{r}\left(k_{\max }\right)$ otherwise, which means that the cutoff frequencies, $\omega_{r}(0)$ and $\omega_{r}\left(k_{\max }\right)$, intersect at $\rho=1 / 7$ [119]. The shaded regions in Fig. 4.7 represent the theoretical predictions of frequency-bands, where the solid and dotted lines represent cutoff frequencies, $\omega_{r}(0)=t_{0}^{-1} c_{r} \sqrt{40 \rho}$ and $\omega_{r}\left(k_{\max }\right)=$ $2 t_{0}^{-1} c_{r} \sqrt{3 \rho+1}$, respectively. In this figure, we also show the theoretical prediction of cutoff frequency of TR mode, $\omega_{t}\left(k_{\max }\right)=t_{0}^{-1} c_{t} \sqrt{10 \rho}$ (the broken line). Our numerical results of the limit, $\omega_{r}(0)$ (the open circles), well agree with the 
model over the whole range of stiffness ratio. In addition, the cutoff frequency of vDOS, $\omega_{c}$ (the open triangles), shows the qualitatively same behavior as the upper cutoff, where $\omega_{c}$ is slightly higher than $\omega_{r}(0)$ because of the finite width around dispersion relation. If the stiffness ratio is large $(\rho>1)$, numerical results of the lower cutoff, $\omega_{r}\left(k_{\max }\right)$ (the open squares), are also explained by the model as $\omega_{r}\left(k_{\max }\right) \sim \sqrt{3 \rho+1}$. However, if the stiffness ratio is small $(\rho<1)$, the lower cutoff deviates from the model and switches to the acoustic branch as $\omega_{t}\left(k_{\max }\right) \sim \sqrt{10 \rho}$. Therefore, different from granular crystals [119], the upper and lower cutoffs of rotational mode in disordered frictional disks never intersect with each other. We also note that the cutoff frequency of TR mode (the crosses) deviates from the theoretical predictions.

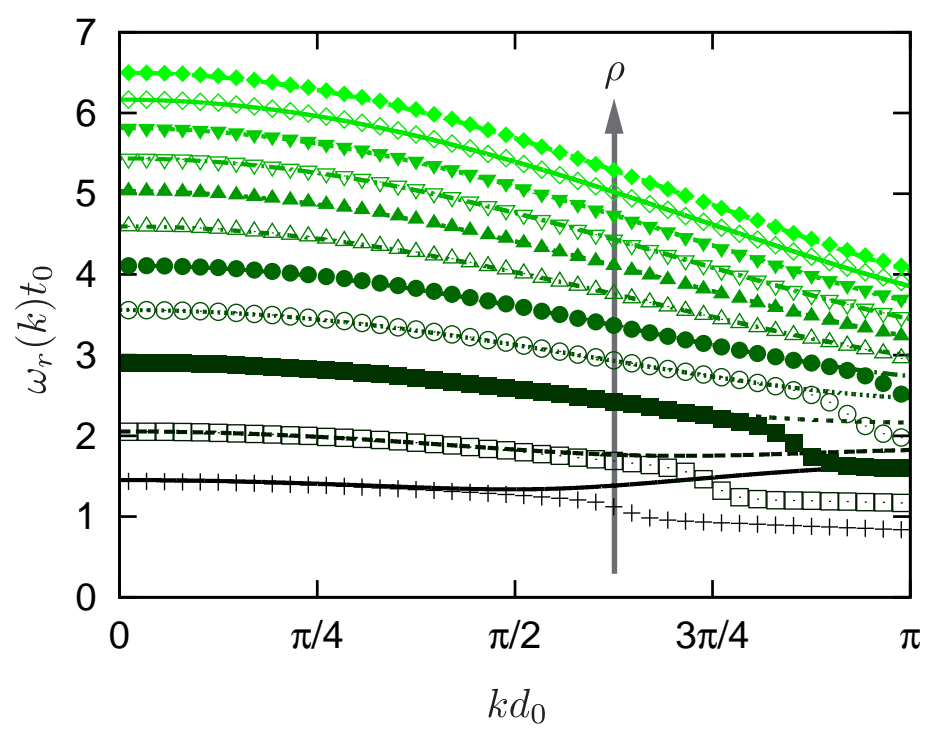

Figure 4.4: (Color online) Dispersion relations of the RT mode, where the symbols are $\omega_{r}(k)$ in Eq. (4.3) and lines are theoretical predictions, Eq. (4.5). The stiffness ratio increases from $\rho=0.1$ to 2.0 as indicated by the arrow, where the area fraction is given by $\phi=0.9$. 

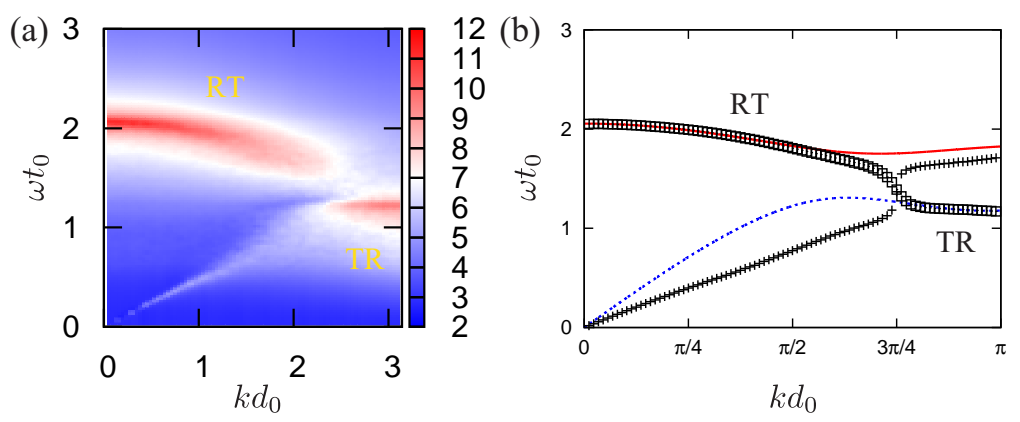

Figure 4.5: (Color online) (a) A three dimensional plot of logarithm of power spectrum, $\log S_{r}(\omega, k)$. (b) Dispersion relations obtained from the VAFs, where the crosses and open squares are $\omega_{t}(k)$ and $\omega_{r}(k)$ in Eq. (4.3), respectively. The dotted and solid lines represent the models, Eqs. (4.4) and (4.5), respectively. In both (a) and (b), the area fraction and stiffness ratio are given by $\phi=0.9$ and $\rho=0.2$, respectively.
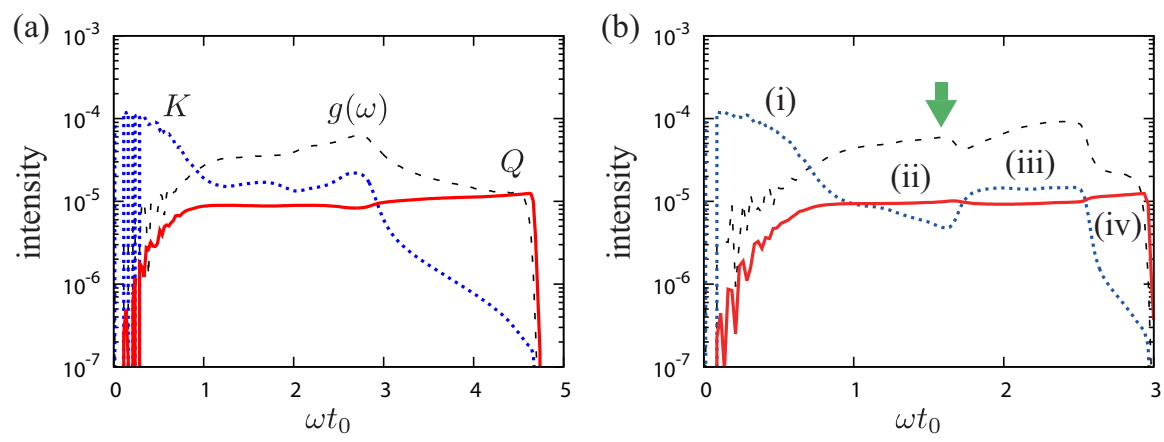

Figure 4.6: (Color online). Semi-logarithmic plots of intensities of $K$ (the dotted lines), $Q$ (the solid lines), and $g(\omega)$ multiplied by $5 \times 10^{-3}$ (the dashed lines), where the stiffness ratios are given by (a) $\rho=1$ and (b) 0.4 . The green vertical arrow in (b) indicates the second peak of vDOS as in Fig. 4.1. 


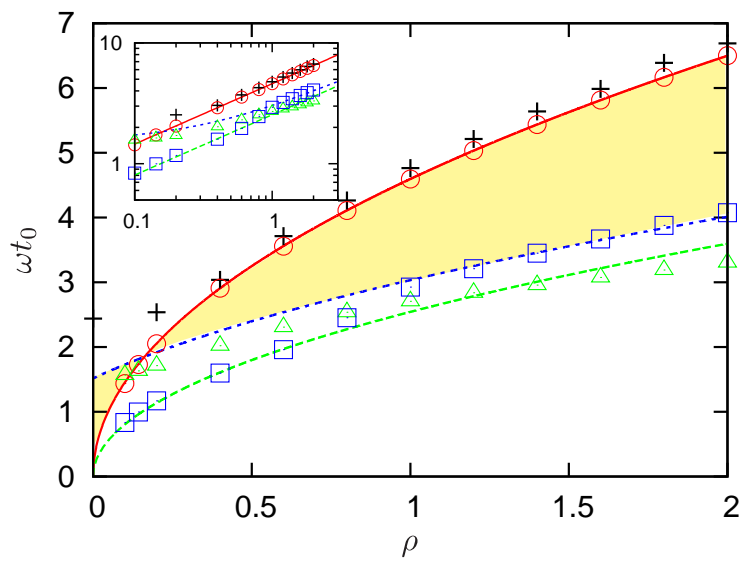

Figure 4.7: (Color online) Frequency-bands of rotational mode, where the (red) solid, (blue) dotted, and (green) dashed lines are theoretical predictions of $\omega_{r}(0)$, $\omega_{r}\left(k_{\max }\right)$, and $\omega_{t}\left(k_{\max }\right)$, respectively. The shaded regions represent the frequency-bands, $\omega_{r}(0)<\omega<\omega_{r}\left(k_{\max }\right)$ for $\rho<1 / 7$ and $\omega_{r}\left(k_{\max }\right)<\omega<\omega_{r}(0)$ otherwise. The open circles and squares are numerical results of cutoff frequencies, $\omega_{r}(0)$ and $\omega_{r}\left(k_{\max }\right)$, respectively. The crosses are the cutoff frequencies, $\omega_{c}$, estimated from vDOS (Fig. 4.1), while the open triangles are numerical results of the cutoff frequency, $\omega_{t}\left(k_{\max }\right)$. Here, the area fraction is fixed to $\phi=0.9$ and the inset displays a double-logarithmic plot. 


\subsection{Discussion and Conclusion}

In this study, we have numerically investigated sound in disordered frictional disk systems. From the dynamical matrix, we find that a shoulder of vDOS extends into the high frequency regime and the cutoff frequency greatly increases with increasing stiffness ratio, $\rho$ (or tangential forces at comparable deformation as normal). Numerically solving the equations of motion, we demonstrate the evolution of L, TR and RT elastic standing waves. Then, vibrational motions of the disks are analyzed by VAFs, where fits to a damped oscillation provide clear dispersion relations (central points in fixed $k$ spectra). Rotational velocities usually for our data $(\rho>0.1)$ show very fast oscillation and a quicker decay than translational ones. This means that rotations are closely related to the shoulder of vDOS and are strongly attenuated/scattered by disorder in the configurations. The power spectra of L, TR and RT velocities show us a nice alternative view of dispersion relations, where the dispersion relations have a finite width in the spectra (due to activation of more than one eigenmode). The optical-like branch of the RT mode spans a high frequency region, corresponding to the shoudler of vDOS and indicates fast oscillations of rotational velocities.

One can explain the dispersion relation of the RT mode by a modified lattice based model. The optical like branch is perfectly described by the model if the stiffness ratio is large, $\rho>1$, i.e. if tangential forces are strong enough. On the other hand if the stiffness ratio is small, $\rho<1$, the dispersion relation switches to the acoustic branch, where numerical results are also well captured by the model. The switching of the RT mode is the origin of the second peak in the vDOS. In addition, we find that the upper and lower limit frequencies do not reverse because of the switching of the RT with TR modes.

In conclusion, rotational sound exhibits characteristic optical-like dispersion relations even in disordered systems. The optical-like branch is well described by the lattice-based model in the case that tangential forces are strong enough and in any case for small enough $k$ (large enough wavelength). On the other hand, if the tangential stiffness is comparable with or smaller than the normal stiffness, it changes to the acoustic branch at a characteristic length scale, indicating that the configurational disorder enables the acoustic behavior of disks'rotations at small enough scales.

In our numerical simulations, we model both the normal and tangential forces between the disks in contact by elastic springs. Thus, the standing waves presented are purely elastic so that total energy is conserved throughout simulations, where the decay of VAFs is solely caused by scattering attenuation 
(and not by energy dissipation). However, in reality, dissipative forces, e.g. the Coulomb (or sliding) friction and viscous forces, also exist between the disks in contact. To take into account such dissipation of energy, we need some generalizations of our model as in $[79,185]$. Therefore, effects of these dissipative forces on our results are left to future work. Similarly, it is interesting to study how other interaction forces, e.g. the rolling resistance [119] and the attractive interaction due to capillary bridges in wet granular material [29], affect the results. Moreover, the influence of microstructre [175], e.g. size-distributions and polydispersity, on the rotational sound is also important.

For practical purposes, numerical studies in three dimensions are crucial, where an additional degree of freedom, i.e. twisting motion of frictional spheres in contact, enables pure rotational (R) mode [119, 120]. Therefore, further studies are needed to clarify how configurational disorder affects the L, TR, RT, and $\mathrm{R}$ modes in three-dimensional granular media. In addition, not only dispersion and scattering attenuation, but also wave diffusion [76] and localization phenomena $[72,152]$ are important aspects of sound in granular material. 


\section{Chapter 5}

\section{Long Wavelength Coherent Pulse of Sound Propagating in Granular Media}

Don't wait for the last wave. Ride the first wave and you will achieve your goals.

Maria Powell

A mechanical wave or vibration propagating through granular media exhibits a specific signature in time, a coherent pulse or wavefront arrives first with multiply scattered waves (coda) arriving later. The coherent pulse is system configuration independent i.e. it depends only on the bulk properties of the disordered granular sample, the sound wave velocity of the granular sample and hence bulk and shear moduli. The coherent wavefront attenuates (decreases in amplitude) and broadens with distance from its source. The pulse attenuation and broadening effects are affected by disorder (polydispersity; contrast in size of the granules) and have often been attributed to dispersion and scattering. To study the effect of disorder and initial amplitude (non-linearity) of the pulse imparted to the system on the coherent wavefront, numerical simulations have been carried out on one-dimensional sets of spherical particles (granular chains). The interaction force between the particles is given by a Hertzian contact model. The sizes of particles have been selected 
randomly from a Gaussian distribution, where the standard deviation of this distribution is the relevant disorder parameter, which quantifies the effect of disorder on the coherent wavefront. Since, the coherent wavefront is system configuration independent, ensemble averaging has been used for improving the signal quality of the coherent pulse and removing the multiply scattered waves. The results concerning the width of the coherent wavefront have been formulated in terms of scaling laws. An experimental construction of photoelastic particles constituting a granular chain as well as a two dimensional arrangement have been used to examine the propagation of this coherent wave packet. ${ }^{1}$

\subsection{Introduction}

Sound propagation through particulate media (granular matter) has been a helpful tool in capturing the very material properties through which it is propagating. This attribute has been extensively used for seismic exploration of oil/gas/mineral reservoirs [174], studying the internal structure of a planetary body (Moon / Earth, [43]) or for geotechnical investigations to determine the material properties [141]. There are various phenomena associated with the sound propagation like multiple scattering, attenuation, geometric spreading, dispersion, coherent back scattering effect, wave localization, etc. [175]. These individual phenomena provide us the tools to measure, evaluate and predict the material properties and hence, enable us to study the material without destroying it (non-destructive testing).

The space time responses of the particles through which the mechanical wave is propagating has a typical signature of its own [76, 93]. The signal has a coherent wave front as the first arrival (in terms of time) and is accompanied by the multiply scattered waves (coda) as shown in the illustration (Fig. 5.1). The coherent wave has long wavelength and low frequency property and the coda has the vice-versa. Although, the multiply scattered part of the signal is extremely phobic to averaging and gets completely removed, the coherent wavefront maintains its shape(width) and size (amplitude) on ensemble averaging in comparison to the signal from a single realization [77]. This property indicates that the coherent wavefront contains information regarding the bulk

\footnotetext{
${ }^{1}$ Based on: Shrivastava, R. K., Thomas, A., Vriend, N., \& Luding, S. (2017). Long Wavelength Coherent Pulse of Sound Propagating in Granular Media. World Academy of Science, Engineering and Technology, International Journal of Mechanical, Aerospace, Industrial, Mechatronic and Manufacturing Engineering, 11(10), 1694-1701.
} 
property of the system, e.g. Bulk Moduli, shear moduli, primary wave velocity (P-wave), co-ordination number etc. [110].

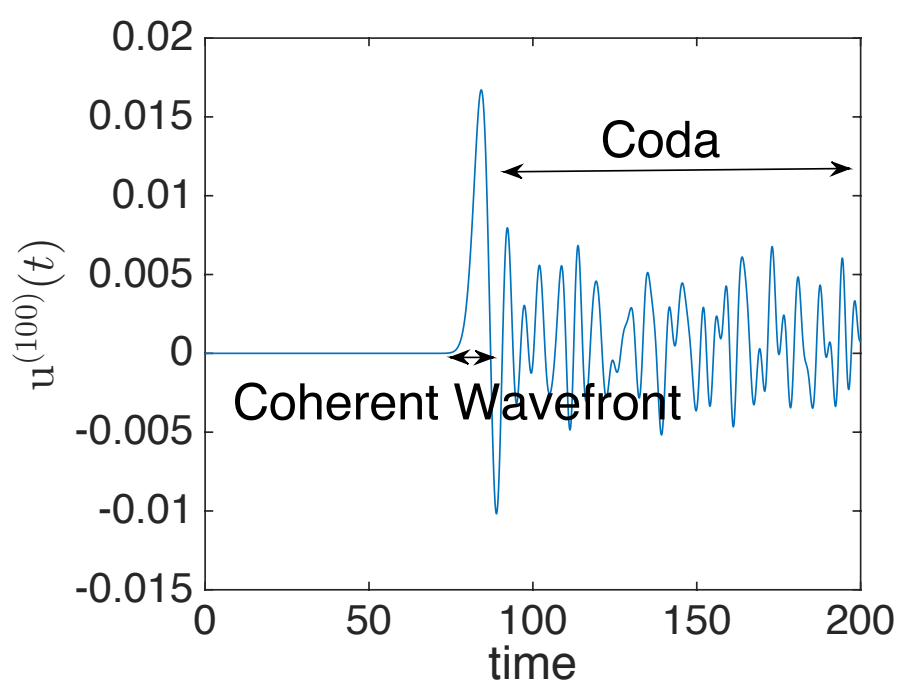

Figure 5.1: Wave propagating in a one-dimensional chain; displacement response of $100^{\text {th }}$ particle, initial impulse $v_{o}=0.1$ plotted against dimensionless time (Section 5.2), disorder parameter $\xi=0.2$ (Section 5.2.5)

In [93], it is shown that the propagating coherent wavefront (pulse) attenuates and "broadens" as it propagates along a medium, this "broadening" is affected by the disorder in particle sizes as well as by the propagation length. We investigate this observation and found some agreements with the scaling parameters observed in the aforementioned work. This observation indicates a potential application for determining the length of the system or the granular size distribution of the medium. However, in [177], it was observed that the space time responses for a set of particles with nonlinear repulsive interaction force (when the amplitude of the propagating wave is high) is significantly different from the ones obtained when the inter-particle forces are linear in nature even if there is no opening and closing of contacts (non-occurrence of sonic vacuum, [136]). The aforementioned observation and the previous work have paved a path for the question as to what happens to this "coherent wavefront/pulse" when it propagates through a not so consolidated granular media (with less pre-compression or high wave amplitude; inter-particle forces vary 
non-linearly with overlap)? The inter-particle forces exhibit a departure from the linear towards non-linear nature, in this scenario does the pulse broadening display similar behavior with length or disorder? In the present case, the impulse amplitude has been varied and its effect has been investigated in the following sections. Also, the coherent wavefront has been instrumental in determining the velocity of sound in a medium which is interconnected with bulk and shear moduli, some light has also been shed on this aspect with Hertzian inter-particle forces taken into consideration.

A one dimensional chain of particles having linear and nonlinear repulsive (Hertzian) interaction forces have been used as models for analyses (Fig. 5.2). The analyses is focused on studying impulse propagation along the chains. The choice of one dimensional chain was motivated by the existence of force chains in granular materials, these force chains act as pathways for sound wave propagation and also support large stresses of the system [25, 146]. Section II contains the micro-mechanical model of the one-dimensional chain describing the linear and non-linear repulsive interaction forces used in the model, similar model has been used in $[98,177,215]$. The disorder in the system is in the form of mass distribution, it has also been discussed in the aforementioned section. Section III contains the numerical results obtained from the simulations of impulse propagation in granular chains, both single realization and ensemble-averaged space time responses have been taken into consideration and discussed upon. Section IV contains the experimental construction of a granular chain using photoelastic elastic discs, it serves as an outlook for experimental validation of numerical results. Section V presents the conclusion along with issues associated with present subject matter requiring further research work.

\subsection{Micromechanical Model: Granular Chain}

An $N+2$ particles long, pre-compressed granular chain of particles has been taken into consideration. $F_{(i, j)}$ is the repulsive interaction force experienced by neighboring particles $i$ and $j, F_{(i, j)} \propto \delta_{(i, j)}^{(1+\beta)}$, where $\delta$ is the non-dimensionalized dynamic inter-particle overlap between the neighboring particles. $\delta_{(i, j)}=\Delta_{(i, j)}-$ $\left(u^{(j)}-u^{(i)}\right)^{(1+\beta)}, \Delta_{(i, j)}$ is the non-dimensionalized initial static overlap due to precompression. $u^{(i)}$ is the non-dimensionalized displacement of the $i^{\text {th }}$ particle relative to the initial static pre-compressed state. Hence,

$$
F_{(i, j)}=\kappa_{(i, j)}\left(\Delta_{(i, j)}-\left(u^{(j)}-u^{(i)}\right)\right)^{(1+\beta)},
$$



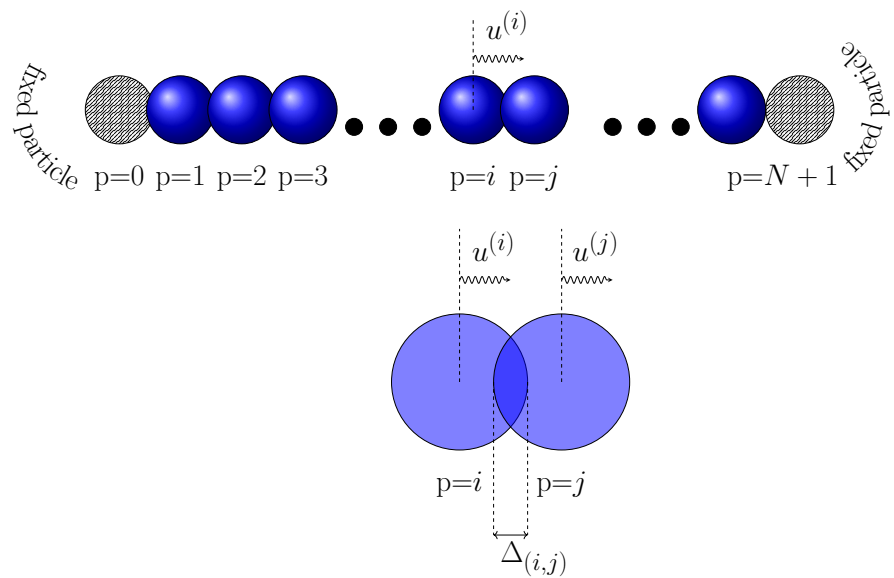

Figure 5.2: Schematic diagram for one dimensional chain.

$\kappa_{(i, j)}$ is the non-dimensionalized stiffness.

\subsubsection{Non-Dimensionalization}

The mass of the $i^{\text {th }}$ particle $\tilde{m}^{(i)}$ is non-dimensionalized by the mean mass $\tilde{m}_{o}, b^{(i)} \equiv \tilde{m}^{(i)} / \tilde{m}_{o}$. The stiffness $\kappa_{(i, j)}$ is non-dimensionalized by characteristic stiffness ( $\kappa_{o}$; stiffness between same size neighboring particles). The displacement of the $i^{\text {th }}$ particle is non-dimensionalized by $\tilde{\Delta}_{o}, u^{(i)} \equiv \tilde{u}^{(i)} / \tilde{\Delta}_{o}$, where $\tilde{\Delta}_{o}$ is the initial static overlap when all the particles have uniform characteristic stiffness $\tilde{\kappa}_{o}$. The initial static overlap for polydisperse particles $\left(\Delta_{(i, j)}\right)$ is also non-dimensionalized by $\tilde{\Delta}_{o}, \Delta_{(i, j)} \equiv \tilde{\Delta}_{(i, j)} / \tilde{\Delta}_{o}$. Time is non-dimensionalized by the characteristic time $\tilde{t}_{c}, t \equiv \tilde{t} / \tilde{t}_{c}$, where $\tilde{t}_{c}=\sqrt{\frac{\tilde{m}_{o}}{\tilde{\kappa}_{(i, j)}}}$.

\subsubsection{Nonlinear Equation of Motion}

The nonlinear equation of motion of particle $i=1$ to $N$ are written as:

$$
\begin{aligned}
b^{(i)} \frac{\mathrm{d}^{2} u^{(i)}}{\mathrm{d} t^{2}}= & -\kappa_{(i, i+1)}\left\{\Delta_{(i, i+1)}-\left(u^{(i+1)}-u^{(i)}\right)\right\}^{(1+\beta)} \\
& +\kappa_{(i, i-1)}\left\{\Delta_{(i, i-1)}-\left(u^{(i)}-u^{(i-1)}\right)\right\}^{(1+\beta)}
\end{aligned}
$$


with $u^{(0)}$ and $u^{(N+1)}$ being 0 as the $0^{t h}$ and $N+1^{t h}$ particles are fixed.

\subsubsection{Hertzian Equation of Motion}

Substituting $\beta=1 / 2$ in Eq. 5.2 gives the Hertzian equation of motion [91]

$$
\begin{aligned}
b^{(i)} \frac{\mathrm{d}^{2} u^{(i)}}{\mathrm{d} t^{2}} & =-\kappa_{(i, i+1)}\left\{\Delta_{(i, i+1)}-\left(u^{(i+1)}-u^{(i)}\right)\right\}^{(3 / 2)} \\
& +\kappa_{(i, i-1)}\left\{\Delta_{(i, i-1)}-\left(u^{(i)}-u^{(i-1)}\right)\right\}^{(3 / 2)}
\end{aligned}
$$

which is valid for repulsive interactions between spherical particles. Equation 5.3 is solved numerically using Verlet integration scheme. Verlet has been used because of its symplectic nature.

\section{Hertz Contact Model}

For nonlinear repulsive interaction between the particles to be Hertzian, the non-dimensionalised contact stiffness is given by $[91,177]$

$$
\tilde{\kappa}_{(i, j)}=\frac{\tilde{\kappa}_{(i, j)}}{\tilde{\kappa}_{o}}=\sqrt{\frac{2}{b^{(i)}+b^{(j)}}}\left(b^{(i)} b^{(j)}\right)^{(1 / 6)} .
$$

The characteristic time is

$$
\tilde{t}_{c}=\frac{1}{\Delta}{ }_{o}^{1 / 4} \sqrt{\frac{1-v^{2}}{\tilde{E}}}\left[\frac{243 \pi \tilde{\rho} \tilde{m}_{o}^{5}}{2}\right]^{1 / 12} .
$$

The initial overlap during static equilibrium is given by

$$
\Delta_{(i, j)}=\frac{\tilde{\Delta}_{(i, j)}}{\tilde{\Delta}_{o}}=\kappa_{(i, j)}^{-2 / 3}
$$

\subsubsection{Linearized Equation of Motion}

Eq. 5.1 can be expressed as a power series about the initial overlap $\left(\Delta_{(i, j)}\right)$,

$$
\begin{array}{r}
F_{(i, j)}=\kappa_{(i, j)} \Delta_{(i, j)}^{1+\beta}+\kappa_{(i, j)}(1+\beta) \Delta_{(i, j)}^{\beta}\left(\delta_{(i, j)}-\Delta_{(i, j)}\right) \\
+\frac{1}{2} \kappa_{(i, j)} \beta(1+\beta) \Delta_{(i, j)}^{\beta-1}\left(\delta_{(i, j)}-\Delta_{(i, j)}\right)^{2}+\ldots
\end{array}
$$


For small amplitudes of wave propagation (in present scenario, an initial impulse, $v_{o}$ ), the displacement of particles from the initial static overlap condition is small, this can be used to ignore higher order terms in Eq. 5.7 resulting in

$$
F_{(i, j)}=\kappa_{(i, j)} \Delta_{(i, j)}^{1+\beta}-\kappa_{(i, j)}(1+\beta) \Delta_{(i, j)}^{\beta}\left(u^{(j)}-u^{(i)}\right) .
$$

Now, Eq. 5.8 can be used to arrive at linearized equation of motion for a particle $i$,

$$
\begin{aligned}
& b^{(i)} \frac{\mathrm{d}^{2} u^{(i)}}{\mathrm{d} t^{2}} \\
& =\kappa_{(i-1, i)} \Delta_{(i-1, i)}^{\beta}\left[\Delta_{(i-1, i)}-(1+\beta)\left(u^{(i)}-u^{(i-1)}\right)\right] \\
& -\kappa_{(i+1, i)} \Delta_{(i, i+1)}^{\beta}\left[\Delta_{(i+1, i)}-(1+\beta)\left(u^{(i+1)}-u^{(i)}\right)\right] .
\end{aligned}
$$

Using $\beta=1 / 2$ and Eq. 5.6 we get linearized hertzian equation of motion

$$
\begin{aligned}
\frac{b^{(i)}}{(1+\beta)} \frac{\mathrm{d}^{2} u^{(i)}}{\mathrm{d} t^{2}} & =\kappa_{(i+1, i)}^{2 / 3}\left(u^{(i+1)}-u^{(i)}\right) \\
& -\kappa_{(i-1, i)}^{2 / 3}\left(u^{(i)}-u^{(i-1)}\right)
\end{aligned}
$$

Equation (10) for particles $i=1$ to $i=N$ can be assembled in the form of an equation consisting of matrices resulting in

$$
\mathbf{M} \frac{\mathrm{d}^{2} \mathbf{u}}{\mathrm{d} t^{2}}=\mathbf{K u}+\mathbf{f},
$$

$\mathbf{M}$ is a diagonal mass matrix with entries $b^{(1)}, b^{(2)}, b^{(3)}, \ldots, b^{(N)}$ and zero otherwise; $\mathbf{K}$ is the stiffness matrix with diagonal entries $-\left(\kappa_{(i+1, i)}+\kappa_{(i-1, i)}\right) /(1+\beta)$, superdiagonal $\left(\kappa_{(i+1, i)} /(1+\beta)\right)$ and subdiagonal $\left(\frac{\kappa_{(i-1, i)}}{(1+\beta)}\right)$ elements. $\mathbf{f}$ is the external force. For impulse driving, $\mathbf{f}=0$, initial displacement vector of particles $\mathbf{u}_{o}=\left[\begin{array}{llll}0 & 0 & \ldots\end{array}\right]$ and initial velocity vector of particles $\mathbf{v}_{o}=\left[\begin{array}{lll}v_{o} & 0 & . .\end{array}\right]$ (the first particle receiving the impulse). Substituting $\mathbf{A}=-\mathbf{M}^{-1} \mathbf{K}$ and using ansatz $\mathbf{u}=\mathbf{u}^{\prime} e^{i \omega t}$ (assuming normal mode motion of particles) gives

$$
\mathbf{A u}=\omega^{2} \mathbf{u} .
$$

The solution of this equation can be expressed as a superposition of eigenmodes,

$$
\mathbf{u}=\mathbf{S C}^{(1)} \mathbf{G}^{-1} \mathbf{S}^{-1} \mathbf{v}_{o}+\mathbf{S} \mathbf{C}^{(2)} \mathbf{S}^{-1} \mathbf{u}_{o}
$$


where $\mathbf{S}$ is the eigenbasis matrix of $\mathbf{A}$ and contains eigenvectors $\mathbf{s}_{j}$ as column vectors. Each eigenvector has a respective eigenvalue $\omega_{j}^{2}$ associated with it ( $\omega_{j}$ is the eigenfrequency). The set of eigenvectors are orthonormalized by the orhtonormality condition $\mathbf{s}_{(i)}^{\mathrm{T}} \mathbf{M s}_{(j)}=\delta_{(i, j)}$ where $\delta_{(i, j)}$ is the Kronecker delta function. Hence, the displacements individual particles are given by

$$
u^{(i)}(t)=v_{o} \sum_{j=1}^{N} \frac{S_{i j} S_{1 j} \sin \left(\omega_{(j)} t\right)}{\omega_{(j)}} .
$$

\subsubsection{Disorder in the Chains and Ensemble Averaging}

The mass of the particles in a granular chain $b^{(i)}$ have been selected randomly from a normal distribution. The mean of the distribution is 1 and its standard deviation is $\xi$, the disorder parameter of the chain. Similar disorder model has been used in $[9,98,177]$, this disorder model has also been termed as "diagonal randomness" [175] because the diagonal elements of the mass matrix $\mathbf{M}$ are the masses of the particles (Section 5.2.4). In [177] and [98], it has been observed that the space time responses of the particles in different disordered chains are quantitatively similar if the first two moments of the mass distributions are the same.

$$
f^{(n)}(b)=\frac{1}{\xi \sqrt{2 \pi}} e^{\frac{(b-1)^{2}}{2 \xi^{2}}}
$$

where $f^{(n)}(b)$ is the probability distribution function of the mass distribution. To obtain a coherent picture of space time responses (coherent wavefront without the multiply scattered part of the wave), ensemble averaging has been used. Multiple realizations of chains have been averaged with same disorder parameter and same impulse amplitude to study the coherent wavefront propagation characteristics. \langle\rangle is used to represent ensemble averaged physical quantities, $\left\langle u^{(i)}(\tau)\right\rangle$ represents ensemble averaged space time response.

\subsection{Numerical Results and Discussions}

Equations 5.3 and 5.14 have been used to numerically and analytically compute space time responses for 512 particles long granular chains for different disorder parameters $(\xi)$ and for different impulse amplitudes $\left(v_{o}\right)$. The space time responses for single realizations as well as for ensembles with the same $\xi$ 
are used for analyses. The time step $\Delta t=9.7656 \times 10^{-4}$ and the maximum time period is 512 units.

\subsubsection{Ensemble Averaged Space Time Responses and the Co- herent Wavefront}

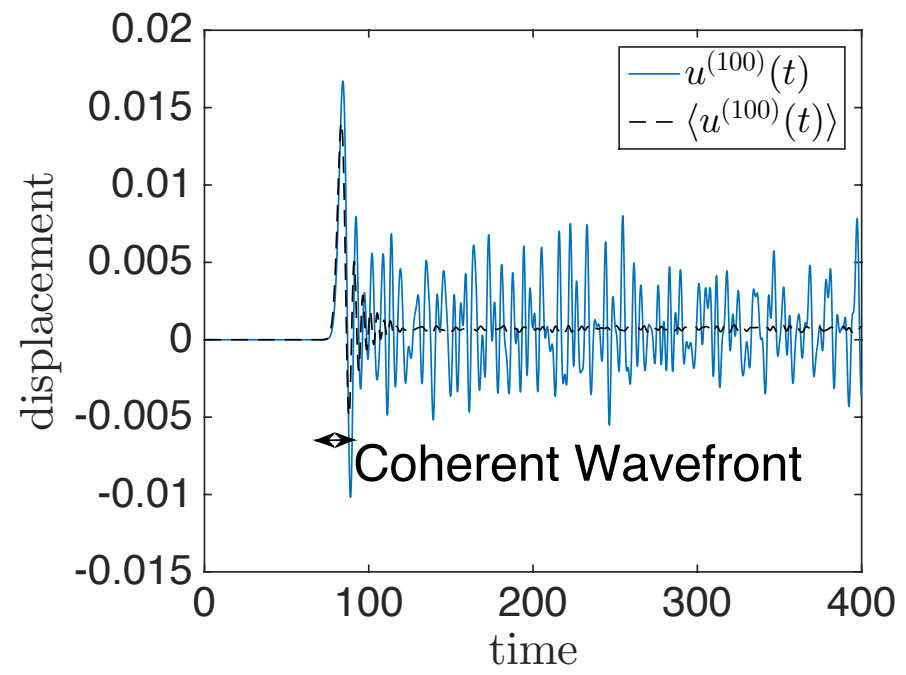

Figure 5.3: Wave propagating in a one-dimensional chain, single and ensemble averaged displacement response of $100^{\text {th }}$ particle, initial impulse $v_{o}=0.1, \xi=0.2$

Fig. 5.3 shows the displacement response of $100^{t h}$ particle in a 512 particles long granular chain for a single realization as well as for ensemble averaged 500 realizations. Ensemble averaging removes the coda, but the coherent wavefront is preserved. As mentioned in Section 5.1, ensemble averaging improves the quality of the coherent wavefront signal which then can be utilized for determining bulk parameters of the material. For further studying the coherent wavefront, it has been characterized by various points as illustrated in Fig. 5.4; a similar methodology has been used previously as well [93], [177], [143].

The point peak signifies the point at which the coherent wavefront achieves the maximum value, $\mathbf{5 0} \%$ signifies the point at which the coherent wavefront achieves the $50 \%$ of the maximum value, similarly with $10 \%$. ZC point signifies the point at which the wavefront crosses the zero value for the first time after 


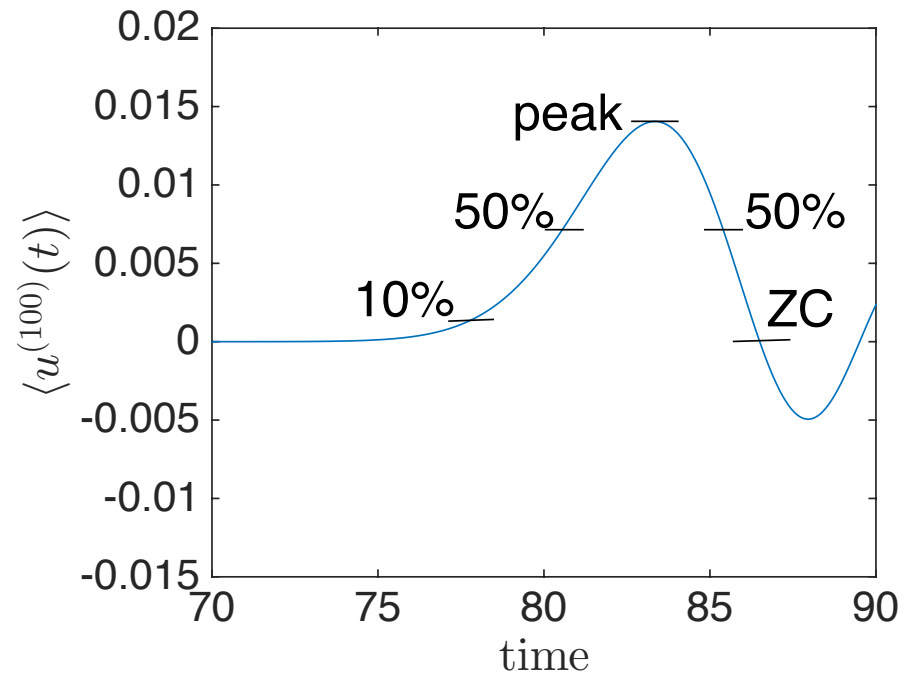

Figure 5.4: Coherent wavefront of the displacement response of $100^{\text {th }}$ particle (zoomed version; see Fig. 5.1), initial impulse $v_{o}=0.1, \xi=0.2$

achieving its maximum value (peak). As it can be observed from Fig. 5.4, the points $\mathbf{1 0} \%$ and $\mathbf{5 0 \%}$ lie on both the sides of the peak of the coherent wavefront, this property is instrumental in measuring the width of the coherent wavefront, the width associated with $50 \%$ is also termed as Full Width Half Maxima (FWHM) and half of the width at $50 \%$ is referred to as Half Width at Half Maxima (HWHM) [weisstein1].

\subsubsection{Width of the Coherent Wavefront}

The width of the coherent wavefront will be evaluated in this section for an ordered chain, disordered chain and then for different impulse amplitudes after adopting a framework for re-scaling the time and amplitude.

\section{Re-Scaling Time and Amplitude}

The framework is similar to the one adopted in [93], it aids in comparison across different simulation parameters as well as while comparison with experimental results. The time and amplitude are scaled by the time of arrival $\left(t_{\text {peak }}\right)$ and 
the amplitude ( $A_{\text {peak }}$ ) of the coherent wavefront respectively. The width of the coherent wavefront for HWHM (50\%) is defined as

$$
W=\frac{t_{\text {peak }}-t_{50 \%}}{t_{\text {peak }}},
$$

where $t_{\text {peak }}$ is the time of arrival or the time at which the coherent wavefront achieves its maximum value. $t_{50 \%}$ is the time at which the coherent wavefront's amplitude is $50 \%$ of the maximum amplitude.

\section{Ordered Chain}

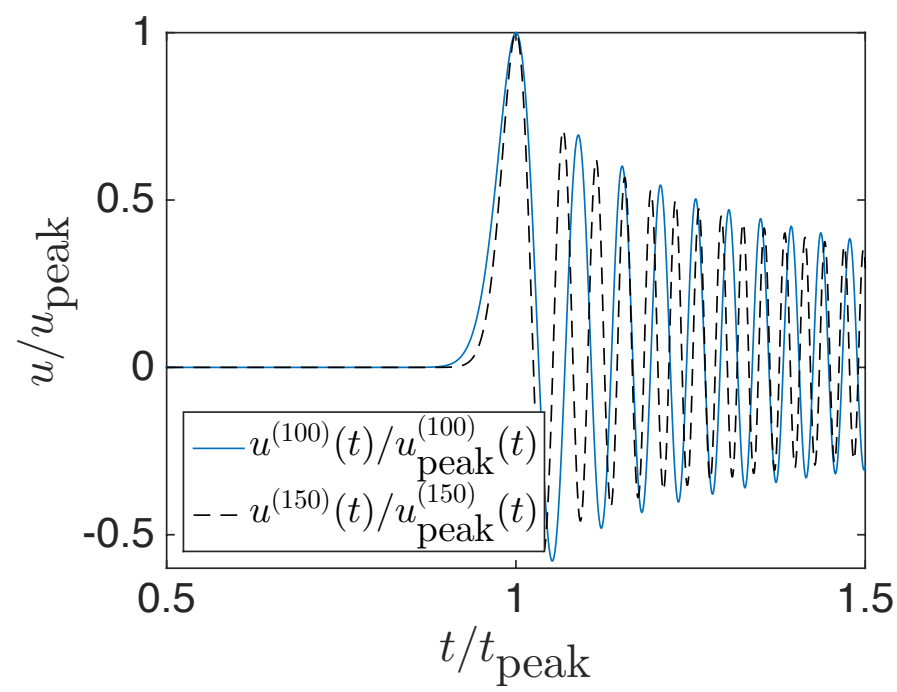

Figure 5.5: Amplitude and time scaled displacement response of $100^{\text {th }}$ and $150^{\text {th }}$ particles, $v_{o}=0.1, \xi=0.0$

Fig. 5.5 contains the amplitude and time scaled displacement response of two different particles along a granular chain, it can be observed that the time of arrival of the coherent wavefront as well as the amplitude has collapsed except the width of the coherent wavefront.

Fig. 5.6 shows the re-scaled displacement response of $100^{\text {th }}$ particle for different $v_{o}$, it shows a perfect collapse of coherent wavefront with same peak 


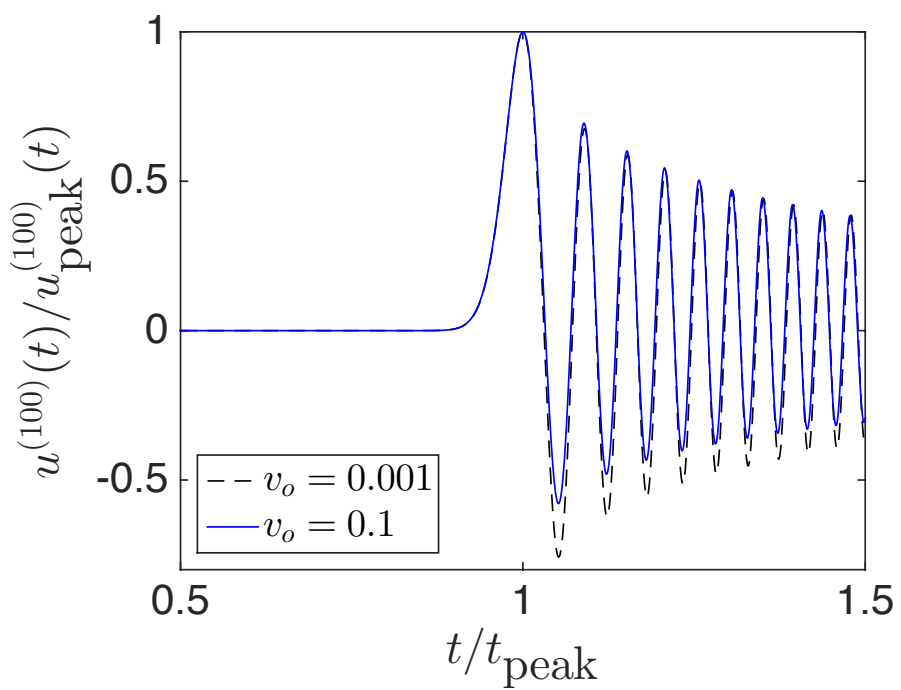

Figure 5.6: Amplitude and time scaled displacement response of $100^{\text {th }}$ particle when $v_{o}=0.001$ and $v_{o}=0.1, \xi=0.0$

amplitude, time of arrival and width as well, indicating that the impulse amplitude has no effect on width $(W)$ of the coherent wavefront in an ordered chain.

\section{Disordered Chain}

Fig. 5.7 displays the space time responses of the $100^{\text {th }}$ particle for different disorder parameters $\left(\xi=0.0, \xi=0.1, \xi=0.2\right.$ and $\left.\xi=0.5, v_{o}=0.1\right)$. From the figure it can be interpreted that the coherent wavefront collapses completely after scaling, but for small disorder parameters, as the disorder increases, the width ceases to remain the same and starts increasing, clearly indicating that the disorder parameter has a role to play in the width of the coherent wavefront.

\section{Width of the Coherent Wavefront}

In [93], it was observed that for ordered chains the Width $(W)$ of the coherent wavefront increases from the distance from the source by a power law relationship, $W \propto L^{-2 / 3}$ which can be observed in our model as well (Fig. 5.8). How- 


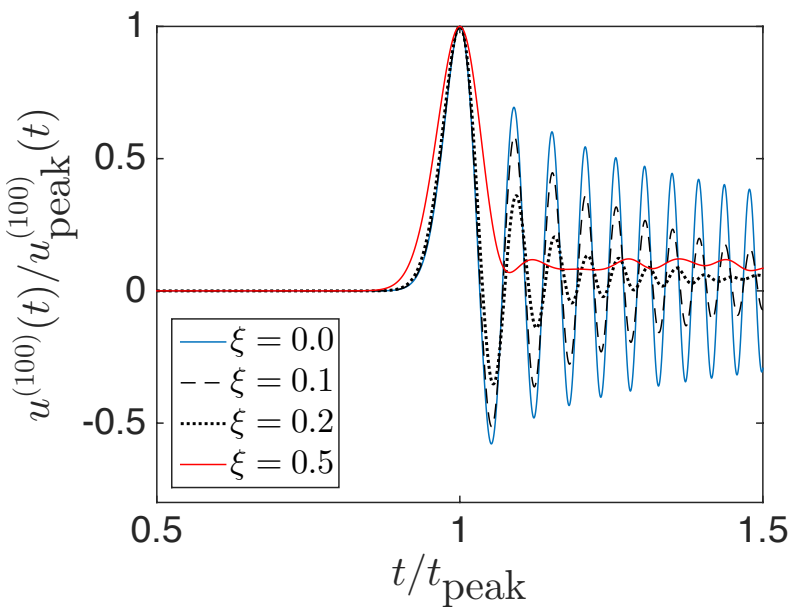

Figure 5.7: Amplitude and time scaled displacement response of $100^{\text {th }}$ particle for different disorder parameters

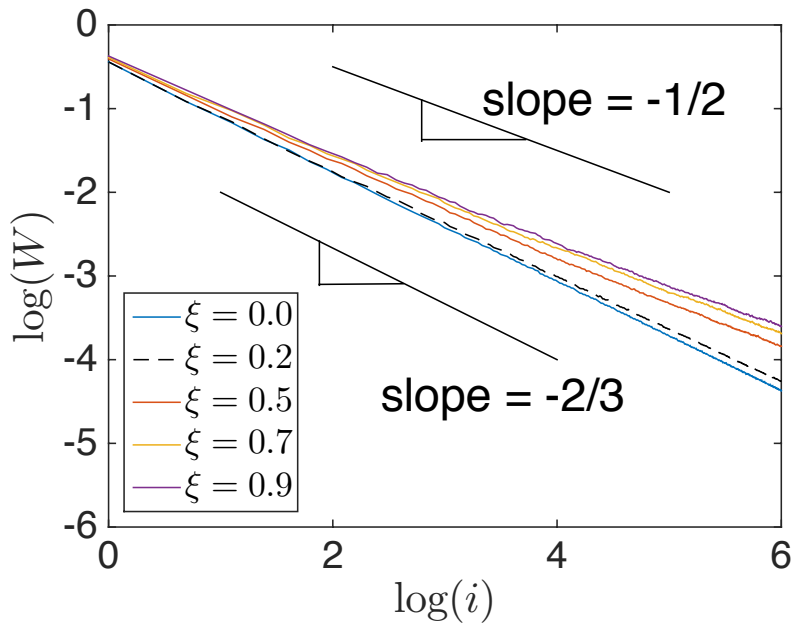

Figure 5.8: Scaled Width $(W)$ of the coherent wavefront for an ordered and disordered chains with distance $i$ from the source. $v_{o}=0.1$ 
ever, in [93] it was proposed that all disordered chains have coherent wavefront propagating with power law relationship of $W \propto L^{-1 / 2}$, but we can see from Fig. 5.8 that for slightly disordered media (e.g. $\xi=0.1,0.2$, etc.), the power law exponent is still $-2 / 3$, it is also observed that the exponenent lies between $-2 / 3$ and $-1 / 2$ for disordered chains and increase in disorder makes the exponent approach $-1 / 2$.

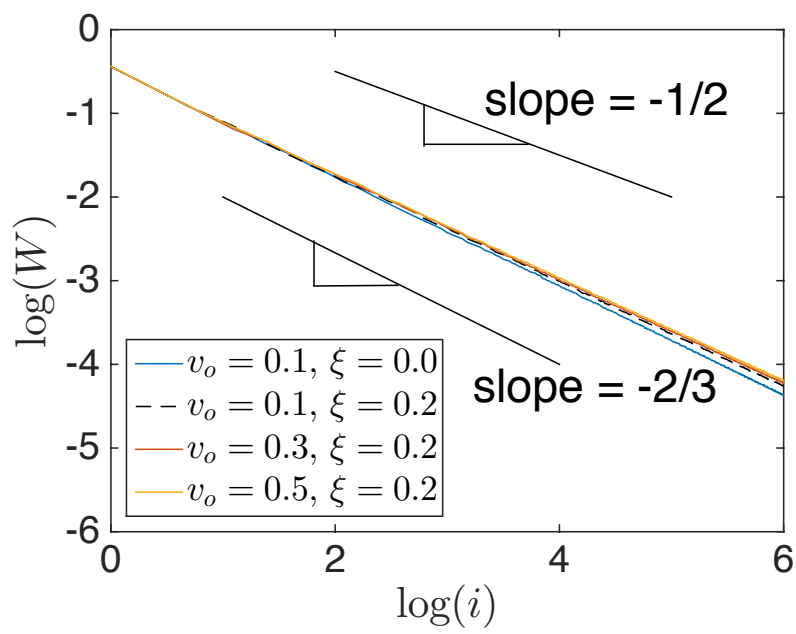

Figure 5.9: Scaled Width $(W)$ of the coherent wavefront for an ordered and a disordered chain $(\xi=0.2)$ with distance $i$ from the source., for varying impulse amplitudes $v_{o}$

From Fig. 5.9 it can be clearly observed that $v_{o}$ has no effect on $W$. The coherent wavefront is not affected by large or small impulse amplitudes.

\subsubsection{Coherent Wavefront Velocity}

The velocity of the coherent wavefront's peak can be calculated by dividing the individual particle number $(i)$ with the time of arrival $t_{\text {peak }}$. Fig. 5.10 shows the same for granular chains with different disorder parameters and and Fig. 5.11 for granular chains experiencing different impulse amplitudes. In both the figures, an acceleration close to the source can be observed which was also observed in [177] and [130]. It is also observed that increase in disorder decreases $v_{\text {peak }}$ and increase in impulse velocity decreases $v_{\text {peak }}$. However, the 


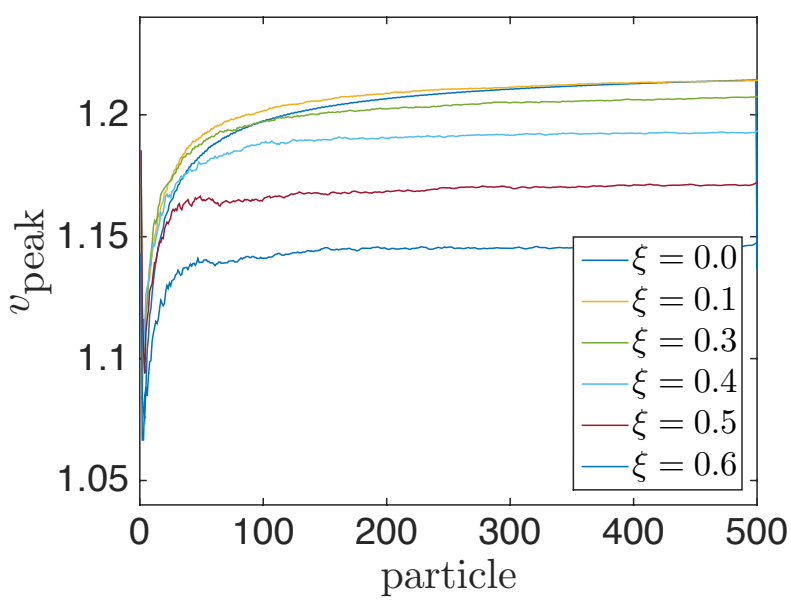

Figure 5.10: Velocity of the peak of coherent wavefront for different particles in a granular chain with varying disorder parameter $\xi$

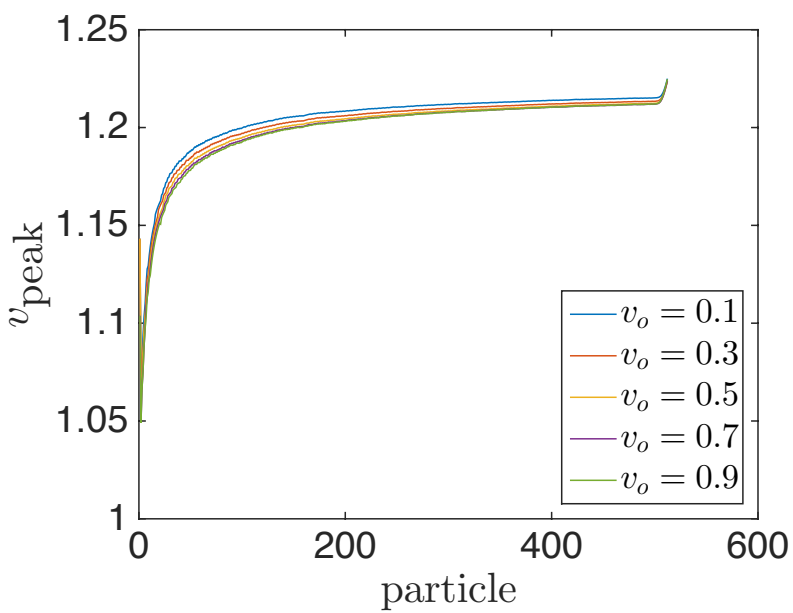

Figure 5.11: Velocity of the peak of coherent wavefront for different particles in a granular chain with varying impulse amplitude $v_{o}$ 


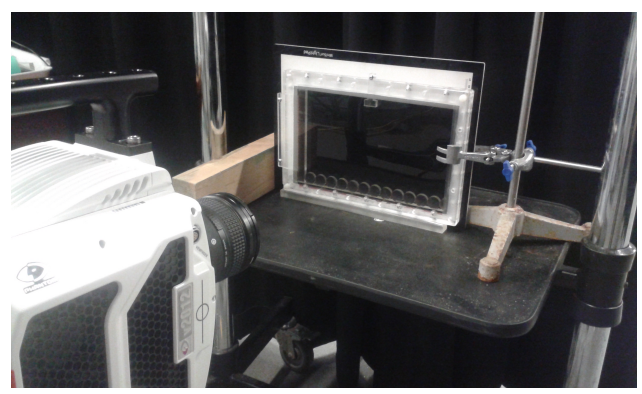

Figure 5.12: Experimental setup

effect of impulse velocity $\left(v_{o}\right)$ is not very significant.

\subsection{Experimental Construction}

To validate the theory described above, the simulations can be reproduced experimentally using discs made out of a photoelastic material, Clear Flex 50 (Smooth-on), to measure the forces at the contacts. Figs. 5.12 and 5.13 show different views of the experimental setup. A chain of 12 photoelastic discs, $2 \mathrm{~cm}$ in diameter and $0.6 \mathrm{~cm}$ deep, were lined up on the bottom of an acrylic container $26 \mathrm{~cm}$ wide, $17 \mathrm{~cm}$ high and $0.8 \mathrm{~cm}$ in depth, one of whose sides was free to move horizontally and act as a plunger on the chain of discs. This container was lit from behind by a MiniSun A4 LED LightPad, and between them a red filter was placed to narrow the range of wavelengths of the light that passed through the photoelastic material. Two opposite circular polarizers were attached on either side of the container, one between the red filter and the first acrylic wall and the second between the other side and a high-speed camera. Thus, the second polarizer would cancel all the light that passes through the first one except for that which was affected by the photoelastic response of the compressed discs. Fig. 5.13 shows the setup when the LightPad is turned on. The acrylic container seems dark due to the opposite polarizers, except for regions within the discs where some red light is transmitted because of the materials photoelastic response to compression. Fig. 5.14 is an example image taken by the camera in this setup. Before an experiment, a heavy block of wood was leaned against the plunger to provide an arbitrary load on the chain of discs. Through a soft tap on the plunger, a pulse can be introduced into the pre-compressed chain, 


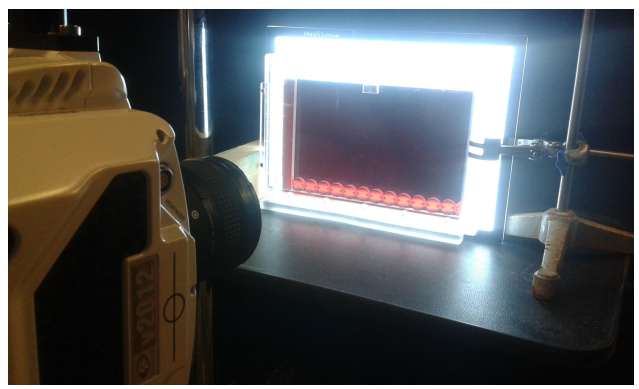

Figure 5.13: Lit experimental setup

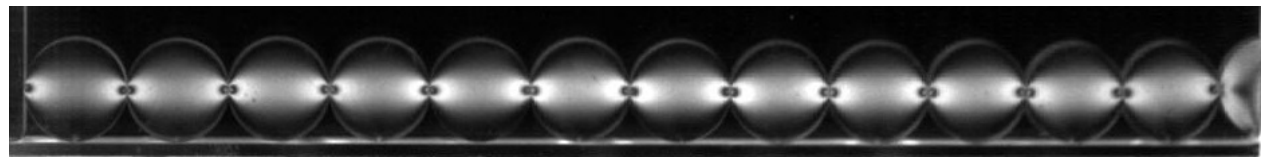

Figure 5.14: One dimensional chain of photoelastic particles

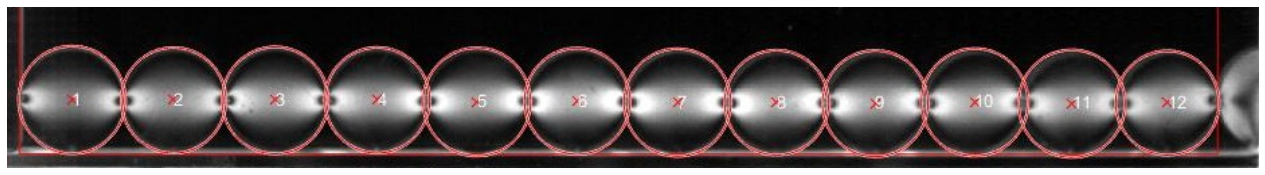

Figure 5.15: One dimensional chain of photoelastic particles with marked circular boundaries of particles

and its progress along the chain can be recorded by the camera. Preliminary experiments show that the pulse takes only a few milliseconds to be transmitted along a disc, so experiments can be recorded at a frame rate of 10000 frames per second to ensure sufficient spatial and temporal resolution. The forces at the contacts on either side of each particle are then computed in the post-processing of each frame.

\subsection{Force Measurements}

First, the measurement of the forces at the contacts between discs was attempted here as a function of their overlap when under compression. At the 


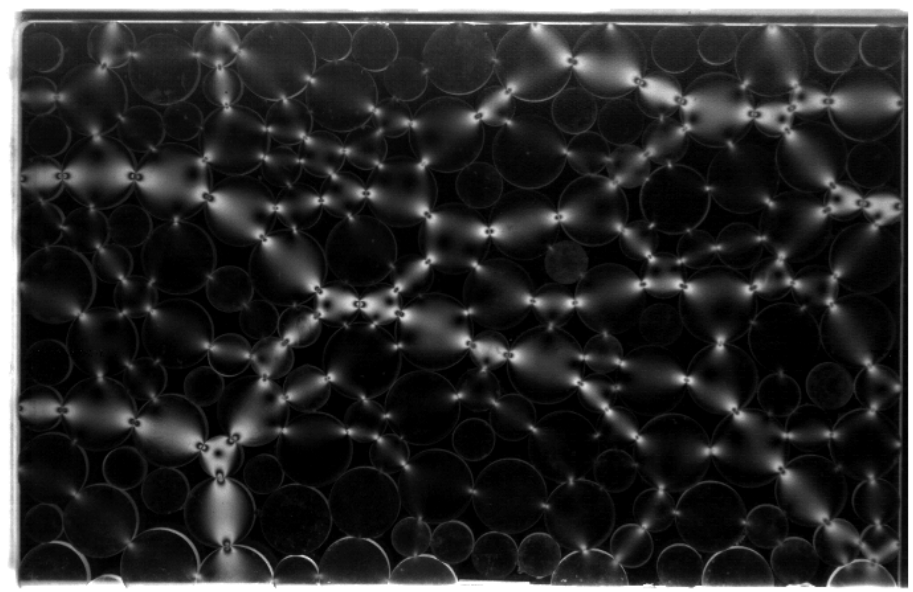

Figure 5.16: Two dimensional setup of photoelastic particles.

disc edges there is a noticeable intensity gradient, and a Hough Transform is applied to each frame returning the center positions and radii of the circles with brightest circumference. Fig. 5.16 superimposes in red the result of this algorithm - the locations and sizes of twelve circles - onto a sample experimental image. The discs are numbered from one to twelve from left to right (in the order in which the pulse travels across them). It can be seen here that the red circles would appear to coincide well with the visible disc edges, because the compression at the contacts is small and does not affect the circularity of most of the discs. Although the discs are under compression, the overlaps between them are only barely perceptible by eye, but the circle-finding method is precise enough to detect a sub-pixels at each contact.

Fig. 5.17a shows the Force response to the kick/impulse of the photoelastic disk chain, the disks were of the same size $(2 \mathrm{~cm})$, the first coherent peak can be easily identified from the responses, the decay of the amplitude and the increase in the width can also be noticed. In Fig. 5.17b, the peak force of all the particles with an exponential fit is plotted, $F p=a \exp ^{-b p}$, where $F p$ is the peak force and $p$ is the particle number, for Fig. 5.17b, $a=0.2821$ and $b=0.01239$.

Fig. 5.18a represents the Force responses of a bidisperse photoelastic disk 


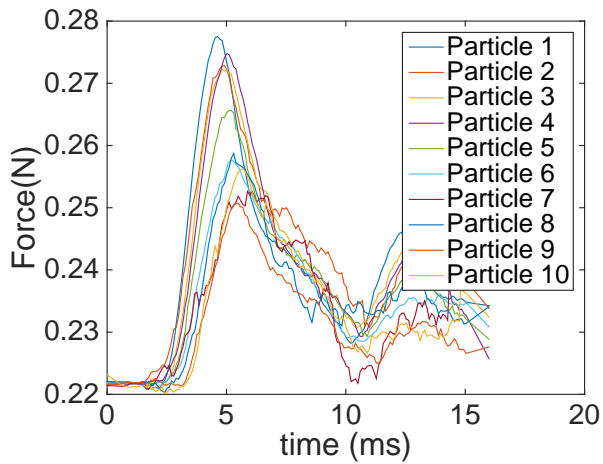

(a) Force response of monodisperse photoelastic disks to an impulse.

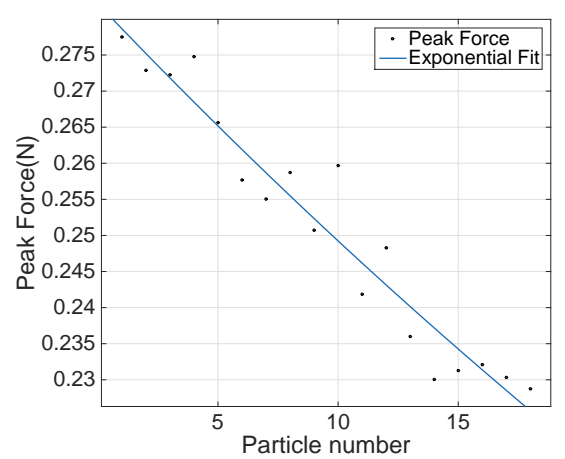

(b) Force response of monodisperse photoelastic disks to an impulse.

Figure 5.17: Monodisperse Disks.

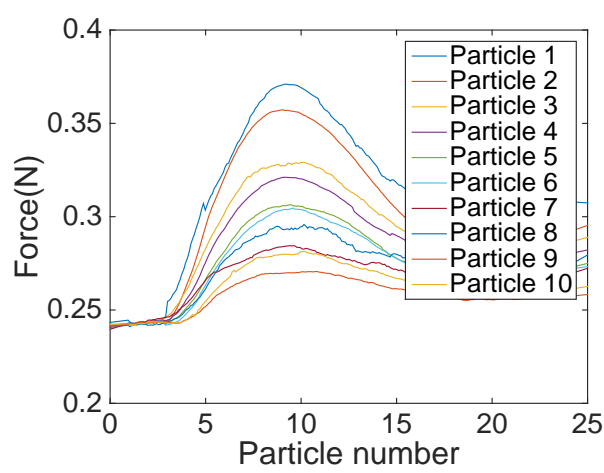

(a) Force response of monodisperse photoelastic disks to an impulse.

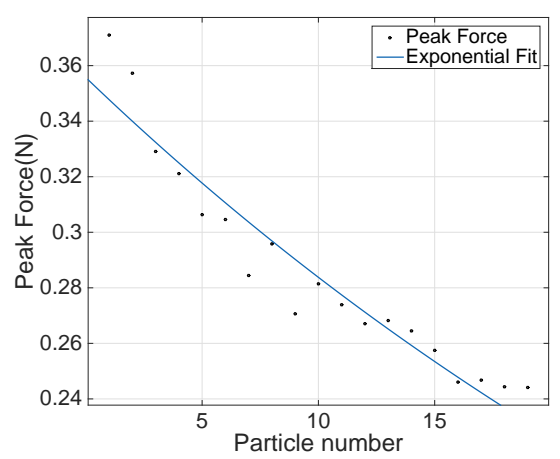

(b) Force response of monodisperse photoelastic disks to an impulse.

Figure 5.18: Bidisperse disks. 
chain $(0.8 \mathrm{~cm}$ and $2 \mathrm{~cm})$, the disks were arranged in one big and one small fashion (one after another). Fig. 5.18b shows the peak forces and their exponential fit, the coefficients $a=0.3557$ and $b=0.02259$, it can be clearly observed that the exponential decay of the peak force has become steeper for bidisperse chain because of disorder, as the exponential coefficient has increased from 0.01239 for monodisperse to 0.02259 for bidisperse.

\subsection{Conclusion}

The coherent wavefront is the long wavelength and low frequency part of a sound signal propagating in granular media. Its short wavelength and high frequency counterpart "coda" is composed of multiply scattered waves, forming the significant part of the sound signal. Thus, on ensemble averaging, the coda gets completely washed out. An elementary granular chain of spherical particles with both linear and nonlinear repulsive interaction forces has been used as a model (Section 5.2) to study the shape and propagation characteristics of the coherent wavefront. The masses of the particles were selected randomly from a normal distribution, the standard deviation of this distribution quantifies the disorder parameter (Section 5.2.5). Both single realizations and displacement responses averaged over various samples were chosen for analysis. The time and amplitude were re-scaled according to the methodology used in [93] for further analysis (Fig. 5.5 and 5.6). Half Width at Half Maximum (HWHM) of the coherent wavefront was used to quantify its scaled width. $W$ decreases with distance from the source following a power law relationship $W \propto L^{-2 / 3}$ for an ordered chain (Fig. 5.8). However, for disordered chains the power law exponent starts increasing with increasing disorder parameter and tends to approach $-1 / 2$ (Fig. 5.9). Change in impulse magnitude $\left(v_{o}\right)$ has no strong effect on the aforementioned power law relationship. The peak velocity of the coherent wavefront ( $v_{\text {peak }}$ ) was also measured and it was observed that increase in disorder considerably decreases the peak velocity and increase in impulse magnitude slightly decreases the peak velocity (Figs. 5.10 and 5.11). An experimental set-up of a granular chain comprising of photoelastic disks was also discussed to validate or challenge the numerical results.

The power law relationship exhibited by the coherent wavefront can be exploited to know the structure or disorder of the system through which sound signals propagate. The attenuation of the coherent wavefront got removed due to the scaling of the amplitude, but its connection with multiple scattering and hence, the coda require further study. 


\section{Chapter 6 \\ Conclusions and Outlook}

When young Galileo, then a student at Pisa, noticed one day during divine
service a chandelier swinging backwards and forwards, and convinced
himself, by counting his pulse, that the duration of the oscillations was
independent of the arc through which it moved, who could know that this
discovery would eventually put it in our power, by means of the pendulum,
to attain an accuracy in the measurement of time till then deemed
impossible, and would enable the storm-tossed seaman in the most distant
oceans to determine in what degree of longitude he was sailing?

Hermann von Helmholtz

The objective of this thesis was to model, understand and predict mechanical waves/sound waves in disordered media. The in depth review in Chapter-1 shows the vastness and numerous frontiers for research on mechanical wave propagation, which unfortunately cannot be encompassed by a single thesis. A smart and elegant approach is step-by-step using simple models and understand one aspect at a time. The material chosen for the study is Granular Media, not only because of its ubiquity but also because it is a paradigm for complex disordered media and the unique characteristics exhibited by various physical phenomena associated with mechanical waves in it (dispersion, scattering attenuation, intrinsic attenuation, diffusion, weak localization, energy transfer across different frequencies, etc.).

Continuum Theoretical Models and numerical techniques like F.E.M, F.D.M, etc. have been helpful in modeling wave propagation, but both have their limitations. Continuum Theoretical Models like Effective Medium Theory (E.M.T) 
cannot quantitatively resolve (take into account during analyses) the microstructure of the disordered media, whereas, all numerical techniques are very computationally expensive but feasible, even when dealing with seismic scales. Particle scale models like D.E.M can provide very detailed and accurate microstructure, but also are much more computationally expensive while modeling real systems. Hence, there is a need for an intermediate model which can be used to ease the computational cost and take into account the relevant microstructure. Stochastic models can assist in such an endeavor, which are based on various previously mentioned physical phenomena associated with mechanical waves, paving the way "Towards Stochastic and Deterministic Modeling of Mechanical Waves in Disordered Media".

To isolate the longitudinal mode of mechanical waves and avoid nonlinearity due to opening and closing of contacts, a pre-compressed one dimensional granular chain of particles has been used in Chapters 2, 3 and 5. The prime motivation behind this selection is the existence of disorder and "force chains" in consolidated granular media which act as backbone/pathway for sound propagation. One advantage is the ease in analytically modeling discrete elements/particles interacting repulsively with linearized coupling for small amplitudes of perturbation and non-linear Hertzian coupling for higher amplitudes.

In Chapter-2, it is observed that the scaled standard deviation of the mass probability distribution of the elements/particles $(\xi)$ of the granular chain can be identified as the relevant disorder parameter. Chains with normal, binary and uniform distribution exhibited quantitatively identical signal transmission characteristics for impulse propagation as long as the first two scaled moments of the distribution were the same and $\xi$ is not too large. The wave speed shows non-monotonous dependence on $\xi$ (for weak disorder the wave speed increases and then decreases for stronger disorder); this occurs because of two opposing effects: increase of wave speed takes place close to the source (this effect is also observed in 3D systems) while increase in disorder decreases the wave speed. An effective weighted dispersion relation is formulated by using the first moment of total eigenmodal energy with frequency, which shows that the cutoff frequency of the medium slightly decreases with increase in disorder. It is also observed that above the low pass frequency band, a wave packet associated with a particular frequency becomes evanescent after traversing a disorder and frequency dependent localization length.

Chapter-3 explores the diffusive versus ballistic characteristics of energy propagation in the granular chain and a stochastic model is proposed, going beyond, but based on deterministic theory. For impulse energy input, the center of energy propagates ballisticalchromely in an ordered chain and is more 
diffusive-like in a disordered chain. The energy becomes localized close to the source at longer time periods because of coherent backscattering of mechanical waves. A standing wave initial condition is used to insert energy into a particular wavenumber and hence, observe the transfer of energies across different wavenumbers. It is observed that energy in higher wavenumbers decays faster than energy in lower wavenumbers before it attains a steady state constant value at large times. This has been used to formulate a Master Equation, a stochastic model to be used for continuum analyses associated with larger systems, which contains information about the micro-structure in the form of the components of the transfer matrix, but much less degrees of freedom.

Complementing the study of chains, disordered frictional two-dimensional disk systems are investigated numerically in Chapter-4 with focus on the rotational waves (that do not exist in 1D). To avoid order (crystallization), a 50:50 mixture of disk types has been used for this purpose (bidisperse). The dynamical matrix is calculated for this system and is used to obtain the vibrational density of states (vDOS). With increase in the stiffness ratio of the disks (ratio between the tangential and normal stiffness), the spread of the shoulder of vDOS in the high frequency regime increases as well, accompanied by an increase in the cut-off frequency. The vibrational motion of the disks is studied using Velocity Auto-Correlation Functions (VAF); the fits to the damped oscillations (where damping is purely due to scattering attenuation) give clean dispersion relations. From the dispersion relations, the rotational mode can be observed to feature a high frequency optical-like branch corresponding to the previously mentioned high frequency regime shoulder of vDOS. A modified lattice based continuum model is able to describe this optical like branch if the stiffness ratio is large; the dispersion relation switches to acoustic branch when the stiffness ratio is small or comparable to normal stiffness, which is also captured by the model.

As introduced and discussed in Chapter-1, a typical mechanical waveform consists of a Coherent wavefront (low frequency in nature, withstands ensemble averaging, identifies with the bulk properties of the system like wave velocity, elastic moduli, etc.) and the Coda (multiply scattered waves, get removed by ensemble averaging, identifies with the configuration and micro-structure of the system, high frequency in nature). In Chapter-5, the coherent wavefront characteristics of a propagating impulse along a granular chain is investigated numerically and experimentally. The investigation is done for different initial impulse amplitudes and disorder parameters. A working setup of photoelastic granular chain is introduced to study the propagation of this coherent wavefront. In the numerical investigations, the width of the coherent wavefront is observed to have a power law relationship with distance from the source. Also 
the experimental results show the expected exponential decay of the peak amplitude of the coherent wavefront, where the exponential decay steepens with increase in disorder.

Finally, it can be said that mechanical wave propagation esp. in granular media has many aspects yet to be studied with the hope that this thesis acts as a beacon for further investigations.

Outlook Future studies should adress the following issues:

- Effective weighted dispersion obtained from the eigenmodes in conjunction with 2D FFT can be used to improve signal to noise ratio of signals in Fourier space, following results presented in Sect. 2.3.6.

- The diffusive equation of energy (center of energy, squared width of energy), effective weighted dispersion relation or the Master Equation of energy in terms of wavenumber in conjunction with F.E.M can be utilized for reducing computational costs during modeling seismic scales, following Chapter-3.

- The Master equation can be extended to compute transfer matrices for 3D real systems, which should be calibrated and validated.

- The effect of dissipative forces (Coulomb friction, viscous damping, etc.) and their inter-play with scattering causing dissipative attenuation can be studied by incorporating these features in the contact model between elements/particles/disks/ and go one step further towards 3D spherical and non-spherical particles, following Chapter-4.

- The wave speed can be explored in different media (fluid saturated media, cohesive media, etc.) close to the source and the overlapping effects (increase in wave velocity due to closeness to source and decrease due to strong disorder) should be investigated.

- Validation of 1D D.E.M model with signal responses (wave propagation) from photoelastic granular chains needs to be addressed. 


\section{Appendix A \\ Total Energy Harmonic Evolution}

The Energy of the system (chain) can be calculated by vector multiplications at a particular instance of time, the non-unitary dimension of the vector gives the respective information of the individual particles. The Kinetic Energy of the chain at a particular instant of time is

$$
E_{k i n}(t)=\frac{1}{2} \mathbf{v}^{\mathrm{T}} \mathbf{M v}
$$

Starting from the impulse initial condition in Sect. 2.2.5, using $\mathbf{v}=\mathbf{S C}^{(2)} \mathbf{H a}$ (Eq. (2.29) and (2.30)) and the orthonormality condition $\mathbf{S}^{\mathrm{T}} \mathbf{M S}=\mathbf{I}$ (Eq. (2.21)), where $\mathbf{I}$ is the identity matrix, the above equation becomes

$$
\begin{aligned}
E_{k i n}(t) & =\frac{1}{2}\left(\mathbf{S C}^{(2)} \mathbf{H a}\right)^{\mathrm{T}} \mathbf{M}\left(\mathbf{S} \mathbf{C}^{(2)} \mathbf{H a}\right) \\
& =\frac{1}{2} \mathbf{a}^{\mathrm{T}} \mathbf{H}^{\mathrm{T}}\left(\mathbf{C}^{(2)}\right)^{\mathrm{T}} \mathbf{S}^{\mathrm{T}} \mathbf{M S C} \mathbf{C}^{(2)} \mathbf{H a}=\frac{1}{2} \mathbf{a}^{\mathrm{T}} \mathbf{H}\left\{\mathbf{C}^{(2)}\right\}^{2} \mathbf{H a}=\frac{1}{2} \sum_{j} a_{j}^{2} \omega_{j}^{2} \sin ^{2}\left(\omega_{j} t\right)
\end{aligned}
$$

Since $\mathbf{C}^{(1)}, \mathbf{C}^{(2)}$ and $\mathbf{H}$ are diagonal matrices, hence their transposition are equal to their original matrices. Note that there is no summation convention applied here. The Potential Energy of the chain at a particular instant of time is

$$
E_{\text {pot }}(t)=-\frac{1}{2} \mathbf{u}^{\mathrm{T}} \mathbf{K u}
$$


Using $\mathbf{u}=\mathbf{S C}^{(1)} \mathbf{a}, \mathbf{v}=\mathbf{S C}^{(2)} \mathbf{G a}$, Eq. (2.30), and orthonormality, the above equation can be written as,

$$
\begin{aligned}
E_{\text {pot }}(t) & =-\frac{1}{2} \mathbf{u}^{\mathrm{T}} \mathbf{K u} \\
& =-\frac{1}{2} \mathbf{u}^{\mathrm{T}} \mathbf{M} \frac{\mathrm{d}^{2} \mathbf{u}}{\mathrm{d} t^{2}} \\
& =-\frac{1}{2}\left(\mathbf{S C}^{(1)} \mathbf{a}\right)^{\mathrm{T}} \mathbf{M} \frac{\mathrm{d} \mathbf{v}}{\mathrm{d} t} \\
& =-\frac{1}{2}\left(\mathbf{S C}^{(1)} \mathbf{a}\right)^{\mathrm{T}} \mathbf{M} \frac{\mathrm{d} \mathbf{S} \mathbf{C}^{(2)} \mathbf{H a}}{\mathrm{d} t} \\
& =\frac{1}{2} \mathbf{a}^{\mathrm{T}} \mathbf{C}^{(1)} \mathbf{S}^{\mathrm{T}} \mathbf{M S C} \mathbf{C}^{(1)}\{\mathbf{H}\}^{2} \mathbf{a} \\
& =\frac{1}{2} \mathbf{a}^{\mathrm{T}} \mathbf{H}\left\{\mathbf{C}^{(1)}\right\}^{2} \mathbf{H a}=\frac{1}{2} \sum_{j} a_{j}^{2} \omega_{j}^{2} \cos ^{2}\left(\omega_{j} t\right) .
\end{aligned}
$$

Hence, the Total Energy becomes a sum over all eigenmode energies:

$$
E_{t o t}=\frac{1}{2} \sum_{j} a_{j}^{2} \omega_{j}^{2}
$$

We can see that $E_{t o t}$ is independent of time (the energy of our chain is conserved). This equation (A.5) also gives us energy with respect to different eigenmodes of the chain (if we drop the first summation term). Hence, $E_{t o t}\left(\omega_{j}\right)=$ $\frac{1}{2} a_{j}^{2} \omega_{j}^{2}$. Now, by replacing $\mathbf{u}, \mathbf{v}$, a with their spatial Fourier transform counter parts $\mathscr{U}, \mathcal{V}$ and $\mathscr{A}$ (calligraphic) by using an ansatz in spatial Fourier space as in Eq. (2.19) for Eq. (2.18), we obtain the harmonic total energy (in terms of wavenumber):

$$
E_{t o t}\left(\omega_{j}\right)=\frac{1}{2} \mathscr{A}_{j}^{2} \omega_{j}^{2}
$$

Initial Configuration Dependance: For the example of an initial pulse ( $\delta$ in real space, i.e. sinusoidal in wavenumber k-space), $\mathscr{A}_{j}(k)$ is constituted by $V_{o}(k)$ in Eq. (2.32), a sinusoidal form (wavenumber $k$ ), imparted initially to the particles, making the parameter $\mathscr{A}_{j}$ dependent on $k$; hence, $E_{\text {tot }}$ is $k$ dependent.

$$
E_{t o t}\left(\omega_{j}, k\right)=\frac{1}{2} \mathscr{A}_{j}^{2}(k) \omega_{j}^{2}
$$




\section{Appendix B}

\section{Hertz contact model}

If Hertzian repulsive interaction force is taken into consideration between particles. The contact stiffness is given by ([91], [97])

$$
\tilde{\kappa}_{(i, j)}=\tilde{Y}_{(i, j)}\left[\frac{\tilde{r}_{i} \tilde{r}_{j}}{\tilde{r}_{i}+\tilde{r}_{j}}\right]^{1 / 2},
$$

where

$$
\tilde{Y}_{(i, j)}^{-1}=\frac{3}{4}\left(\frac{1-v_{i}^{2}}{\tilde{E}_{i}}+\frac{1-v_{j}^{2}}{\tilde{E}_{j}}\right) .
$$

$\tilde{E}_{i}$ and $v_{i}$ are the elastic modulus and Poisson's ratio, respectively of the particle $i$. If the particles are made up of the same material, $\tilde{Y}_{(i, j)}$ becomes same for all the contacts,

$$
\tilde{Y}^{-1}=\frac{3}{2}\left(\frac{1-v^{2}}{\tilde{E}}\right) .
$$

The characteristic stiffness of the contact is

$$
\tilde{\kappa}_{o}=\frac{\tilde{E}}{1-v^{2}}\left[\frac{2 \tilde{m}_{o}}{243 \pi \tilde{\rho}}\right]^{1 / 6} .
$$

The characteristic initial overlap becomes

$$
\tilde{\Delta}_{o}=\left(\frac{\tilde{F}_{o}}{\tilde{\kappa}_{o}}\right)^{2 / 3} .
$$


The characteristic time is

$$
\tilde{t}_{c}=\frac{1}{\tilde{\Delta}_{o}^{1 / 4}} \sqrt{\frac{1-v^{2}}{\tilde{E}}}\left[\frac{243 \pi \tilde{\rho} \tilde{m}_{o}^{5}}{2}\right]^{1 / 12} .
$$

The scaled stiffness ratio is

$$
\kappa_{(i, j)}=\frac{\tilde{\kappa}_{(i, j)}}{\tilde{\kappa}_{o}}=\sqrt{\frac{2}{b^{(i) 1 / 3}+b^{(j) 1 / 3}}}\left(b^{(i)} b^{(j)}\right)^{1 / 6} .
$$

The initial overlap during static equilibrium can be formulated as

$$
\Delta_{(i, j)}=\frac{\tilde{\Delta}_{i, j}}{\tilde{\Delta}_{o}}=\kappa_{(i, j)}^{-2 / 3} .
$$




\section{Appendix C}

\section{Matching the first two moments of different distributions (normal, uniform and binary).}

To study only the effect of disorder parameter (standard deviation $\xi$ ) rather than the shape of the distribution on the wave propagation characteristics (Chapter 2 ), the first two moments of normal, uniform and binary distributions have been matched analytically. The raw $n^{t h}$ moment of a distribution is defined as

$$
\tilde{M}_{n}^{(q)}=\int_{-\infty}^{\infty} \tilde{m}^{n} f^{(q)}(\tilde{m}) \mathrm{d} \tilde{m}
$$

where, $f^{(q)}(\tilde{m})$ is the distribution, $q$ is the type of distribution and $\tilde{m}$ is the variable for which the distribution has been defined. $q$ is $n$ for normal distribution, $u$ for uniform distribution and $b i$ for binary distribution. The raw scaled 
moment is defined as

$$
\begin{aligned}
M_{n}^{(q)} & =\frac{\tilde{M}_{n}^{(q)}}{\tilde{M}_{1}^{(q)}}, \\
& =\frac{\int_{-\infty}^{\infty} \tilde{m}^{n} f(\tilde{m}) \mathrm{d} \tilde{m}}{\left(\int_{-\infty}^{\infty} \tilde{m} f(\tilde{m}) \mathrm{d} \tilde{m}\right)^{n}}, \\
& =\frac{\int_{-\infty}^{\infty} \tilde{m}^{n} f(\tilde{m}) \mathrm{d} \tilde{m}}{\left(\tilde{M}_{1}\right)^{n}},
\end{aligned}
$$

(where first raw moment is the average of the distribution $\left(\tilde{M}_{1}\right)$ )

$$
\begin{aligned}
& =\int_{-\infty}^{\infty}\left(\frac{\tilde{m}}{\tilde{M}_{1}}\right)^{n} f(\tilde{m}) \mathrm{d} \tilde{m}, \\
& =\int_{-\infty}^{\infty} b^{n}\left\{\tilde{m}_{o} f(\tilde{m})\right\} \mathrm{d} b, \text { (where } b=\tilde{m} / \tilde{m}_{o} \text { is the scaled mass (Sect. 2.2.1)) } \\
& =\int_{-\infty}^{\infty} b^{n} f(b) \mathrm{d} b \cdot(f(b) \text { is the scaled mass distribution) }
\end{aligned}
$$

In case of particle mass distribution, only positive values can be considered so that the lower limit is to be replaced by zero, which leads to modifications for larger $\xi$.

\section{C.1 Normal Distribution}

The unscaled normal distribution is given as

$$
f^{(n)}(\tilde{m})=\frac{1}{\tilde{\xi}(n) \sqrt{2 \pi}} e^{-\frac{\left(\tilde{m}-\tilde{M}_{1}\right)^{2}}{2(\tilde{\xi}(n))^{2}}},
$$

where, $\tilde{\xi}^{(n)}$ is the standard deviation and $\tilde{M}_{1}$ is the average of the distribution. The scaled normal distribution is given as

$$
\begin{aligned}
f^{(n)}(b) & =\tilde{M}_{1} f^{(n)}(\tilde{m}), \\
& =\frac{1}{\xi^{(n)} \sqrt{2 \pi}} e^{-\frac{(b-1)^{2}}{2\left(\xi^{(n)}\right)^{2}}} .
\end{aligned}
$$

where $b=\tilde{m} / \tilde{M}_{1}$ is the scaled mass and $\xi^{(n)}=\tilde{\xi}^{(n)} / \tilde{M}_{1}$ is the scaled standard deviation which is the disorder parameter for the one dimensional chain. 


\section{C.1.1 First Moment}

The first scaled moment of the normal distribution is given as

$$
\begin{aligned}
M_{1}^{(n)} & =\int_{-\infty}^{\infty} b \frac{1}{\xi(n) \sqrt{2 \pi}} e^{-\frac{(b-1)^{2}}{2\left(\xi^{(n)}\right)^{2}}} \mathrm{~d} b, \\
& =\underbrace{\int_{-\infty}^{\infty}(b-1) \frac{1}{\xi(n) \sqrt{2 \pi}} e^{-\frac{(b-1)^{2}}{2(\xi(n))^{2}}} \mathrm{~d} b}_{\text {non-even power of } \mathrm{b}}+\underbrace{\int_{-\infty}^{\infty} \frac{1}{\xi^{(n)} \sqrt{2 \pi}} e^{-\frac{(b-1)^{2}}{2\left(\xi^{(n))^{2}}\right.} \mathrm{d} b}}_{\text {even power of } \mathrm{b}} \\
& =0+\frac{1}{\sqrt{\pi}} \times \sqrt{\pi} \\
& =1 .
\end{aligned}
$$

Hence, the first scaled moment of the normal distribution is 1 .

\section{C.1.2 Second Moment}

The Gaussian integral (normalizing condition) is given by

$$
\frac{1}{\sqrt{2 \pi\left(\xi^{(n)}\right)^{2}}} \int_{-\infty}^{\infty} e^{-\frac{(b-1)^{2}}{2\left(\xi^{(n)}\right)^{2}}} \mathrm{~d} b=1,
$$


it can be differentiated with respect to $\left(\xi^{(n)}\right)^{2}$ to get

$$
\begin{aligned}
& -\frac{1}{2\left(\xi^{(n)}\right)^{3} \sqrt{2 \pi}} \int_{-\infty}^{\infty} e^{-\frac{(b-1)^{2}}{2\left(\xi^{(n)}\right)^{2}}} \mathrm{~d} b+\frac{1}{\sqrt{2 \pi\left(\xi^{(n)}\right)^{2}}} \int_{-\infty}^{\infty} \frac{(b-1)^{2}}{2\left(\xi^{(n)}\right)^{4}} e^{-\frac{(b-1)^{2}}{2\left(\xi^{(n)}\right)^{2}}} \mathrm{~d} b=0, \\
& \Rightarrow \frac{1}{\sqrt{\left(\xi^{(n)}\right)^{2} 2 \pi}} \int_{-\infty}^{\infty}(b-1)^{2} e^{-\frac{(b-1)^{2}}{2\left(\xi^{(n)}\right)^{2}}} \mathrm{~d} b=\frac{\left(\xi^{(n)}\right)^{2}}{\sqrt{2 \pi\left(\xi^{(n)}\right)^{2}}} \underbrace{\int_{-\infty}^{\infty} e^{-\frac{(b-1)^{2}}{2\left(\xi^{(n)}\right)^{2}}} \mathrm{~d} b}_{\text {Normalizing condition }}, \\
& \Rightarrow \frac{1}{\sqrt{\left(\xi^{(n)}\right)^{2} 2 \pi}} \int_{-\infty}^{\infty} b^{2} e^{-\frac{(b-1)^{2}}{2\left(\xi^{(n)}\right)^{2}}} \mathrm{~d} b=\left(\xi^{(n)}\right)^{2}-\underbrace{\frac{1}{\sqrt{2 \pi\left(\xi^{(n)}\right)^{2}}} \int_{-\infty}^{\infty} e^{-\frac{(b-1)^{2}}{2\left(\xi^{(n)}\right)^{2}}} \mathrm{~d} b}_{\text {Normalizing condition }=1} \\
& +\frac{1}{\sqrt{2 \pi\left(\xi^{(n)}\right)^{2}}} \int_{-\infty}^{\infty} 2 b e^{-\frac{(b-1)^{2}}{2\left(\xi^{(n)}\right)^{2}}} \mathrm{~d} b \\
& \Rightarrow M_{2}^{(n)}=\left(\xi^{(n)}\right)^{2}-1+2 \underbrace{\frac{1}{\sqrt{2 \pi\left(\xi^{(n)}\right)^{2}}} \int_{-\infty}^{\infty} b e^{-\frac{(b-1)^{2}}{2\left(\xi^{(n)}\right)^{2}}} \mathrm{~d} b}_{M_{1}^{(n)}=1}, \\
& \Rightarrow M_{2}^{(n)}=1+\left(\xi^{(n)}\right)^{2} .
\end{aligned}
$$

Taking $\xi^{(n)}=\xi$, the second scaled moment of the normal distribution is $1+\xi^{2}$.

\section{C.2 Binary Distribution}

The unscaled binary distribution is given by

$$
f^{(b i)}(\tilde{m})=\frac{\delta\left(\tilde{m}-\left(\tilde{M}_{1}+\tilde{\xi}^{(b i)}\right)\right)}{2}+\frac{\delta\left(\tilde{m}-\left(\tilde{M}_{1}-\tilde{\xi}^{(b i)}\right)\right)}{2},
$$

The scaled binary distribution is given as

$$
\begin{aligned}
f^{(b i)}(b) & =\tilde{M}_{1} f^{(b i)}(\tilde{m}) \\
& =\frac{\tilde{M}_{1}}{2}\left[\delta\left(\tilde{M}_{1}\left\{\frac{\tilde{m}}{\tilde{M}_{1}}-\left(\frac{\tilde{m}}{\tilde{M}_{1}}-\frac{\tilde{\xi}^{(b i)}}{\tilde{M}_{1}}\right)\right\}\right)+\delta\left(\tilde{M}_{1}\left\{\frac{\tilde{m}}{\tilde{M}_{1}}-\left(\frac{\tilde{m}}{\tilde{M}_{1}}-\frac{\tilde{\xi}^{(b i)}}{\tilde{M}_{1}}\right)\right\}\right)\right] \\
& =\frac{\delta\left\{b-\left(1-\xi^{(b i)}\right)\right\}+\delta\left\{b-\left(1+\xi^{(b i)}\right)\right\}}{2}
\end{aligned}
$$

where $b=\tilde{m} / \tilde{M}_{1}$ is the scaled mass and $\xi^{(b i)}=\tilde{\xi}^{(b i)} / \tilde{M}_{1}$ is the scaled standard deviation which is the disorder parameter for the one dimensional chain. 


\section{C.2.1 First Moment}

The first scaled moment of the distribution is given as

$$
\begin{aligned}
M_{1}^{(b i)} & =\int_{-\infty}^{\infty} b f^{(b i)}(b) \mathrm{d} b \\
& =\int_{-\infty}^{1} b f^{(b i)}(b) \mathrm{d} b+\int_{1}^{\infty} b f^{(b i)} \mathrm{d} b, \text { (Splitting the integral around 1) } \\
& =\int_{-\infty}^{1} b \frac{\delta\left\{b-\left(1-\xi^{(b i)}\right)\right\}}{2} \mathrm{~d} b+\int_{1}^{\infty} b \frac{\delta\left\{b-\left(1+\xi^{(b i)}\right)\right\}}{2} \mathrm{~d} b, \\
& \text { (Probabilty distribution function) } \\
& =\frac{1-\xi^{(b i)}}{2}+\frac{1+\xi^{(b i)}}{2} \\
& =1
\end{aligned}
$$

Hence, the first scaled moment of the binary distribution is 1 .

\section{C.2.2 Second Moment}

The second scaled moment of the binary distribution is given as follows :

$$
\begin{aligned}
M_{2}^{(b i)} & =\int_{-\infty}^{\infty} b^{2} f^{(b i)}(b) \mathrm{d} b \\
& =\int_{-\infty}^{1} b f^{(b i)}(b) \mathrm{d} b+\int_{1}^{\infty} b f^{(b i)} \mathrm{d} b, \text { (Splitting the integral around 1) } \\
& =\int_{-\infty}^{1} b^{2} \frac{\delta\left\{b-\left(1-\xi^{(b i)}\right)\right\}}{2} \mathrm{~d} b+\int_{1}^{\infty} b^{2} \frac{\delta\left\{b-\left(1+\xi^{(b i)}\right)\right\}}{2} \mathrm{~d} b \\
& (\text { Probabilty distribution function) } \\
& =\frac{\left(1-\xi^{(b i)}\right)^{2}}{2}+\frac{\left(1+\xi^{(b i)}\right)^{2}}{2} \\
& =1+\left(\xi^{(b i)}\right)^{2}
\end{aligned}
$$

Taking $\xi^{(n)}=\xi^{(b i)}=\xi$, the second scaled moment of the binary distribution is $1+\xi^{2}$. 
Meftching the first two moments of different distributions (normal, uniform and binary).

\section{C.3 Uniform Distribution}

The unscaled uniform distribution for the mass distribution is given by

$$
f^{(u)}(\tilde{m})= \begin{cases}\frac{1}{2 \tilde{\xi}^{(u)}} & \text { for } \tilde{M}_{1}-\tilde{\xi}^{(u)} \leq \tilde{m} \leq \tilde{M}_{1}+\tilde{\xi}^{(u)} \\ 0 & \text { for } \tilde{m}<\tilde{M}_{1}-\tilde{\xi}^{(u)} \text { or } \tilde{m}>\tilde{M}_{1}+\tilde{\xi}^{(u)}\end{cases}
$$

The value of the mass is $\frac{1}{2 \tilde{\xi}^{(u)}}$ in the interval $\left[\tilde{M}_{1}-\tilde{\xi}^{(u)}, \tilde{M}_{1}+\tilde{\xi}^{(u)}\right]$ and 0 elsewhere. The scaled uniform distribution is given as

$$
\begin{aligned}
f^{(u)}(b) & =\tilde{M}_{1} f^{(u)}(\tilde{m}), \\
& = \begin{cases}\frac{1}{2 \xi^{(u)}} & \text { for } 1-\xi^{(u)} \leq b \leq 1+\xi^{(u)} \\
0 & \text { for } b<1-\xi^{(u)} \text { or } b>1+\xi^{(u)}\end{cases}
\end{aligned}
$$

The scaled masses $(b)$ are selected from $1 \pm \xi$ to approximately p-reserve symmetry about the scaled mean.

\section{C.3.1 First Moment}

The first scaled moment of the distribution is given as

$$
\begin{aligned}
M_{1}^{(u)} & =\int_{-\infty}^{\infty} b f^{(u)}(b) \mathrm{d} b \\
& =\int_{-\infty}^{1-\xi^{(u)}} b f^{(u)}(b) \mathrm{d} b+\int_{1-\xi^{(u)}}^{1+\xi^{(u)}} b f^{(u)}(b) \mathrm{d} b+\int_{1+\xi^{(u)}}^{\infty} b f^{(u)}(b) \mathrm{d} b \\
& =0+\int_{1-\xi^{(u)}}^{1+\xi^{(u)}} b f^{(u)}(b) \mathrm{d} b+0
\end{aligned}
$$

(The distribution is 0 elsewhere except the interval (C.13))

$$
\begin{aligned}
& =\int_{1-\xi^{(u)}}^{1+\xi^{(u)}} \frac{b}{2 \xi^{(u)}} \mathrm{d} b \\
& =\left.\frac{b^{2}}{4 \xi^{(u)}}\right|_{1-\xi^{(u)}} ^{1+\xi^{(u)}} \\
& =1 .
\end{aligned}
$$

Hence, the first scaled moment of the uniform distribution is 1 . 


\section{C.3.2 Second Moment}

The second moment of the distribution is given as

$$
\begin{aligned}
M_{2}^{(u)} & =\int_{-\infty}^{\infty} b^{2} f^{(u)}(b) \mathrm{d} b \\
& =\int_{-\infty}^{1-\xi^{(u)}} b^{2} f^{(u)} \mathrm{d} b+\int_{1-\xi^{(u)}}^{1+\xi^{(u)}} b^{2} f^{(u)} \mathrm{d} b+\int_{1+\xi^{(u)}}^{\infty} b^{2} f^{(u)} \mathrm{d} b \\
& =0+\int_{1-\xi^{(u)}}^{1+\xi^{(u)}} b^{2} f^{(u)} \mathrm{d} b+0
\end{aligned}
$$

(The distribution is 0 elsewhere except the interval (C.13))

$$
\begin{aligned}
& =\int_{1-\xi^{(u)}}^{1+\xi^{(u)}} \frac{b^{2}}{2 \xi} \mathrm{d} b \\
& =\left.\frac{b^{3}}{6 \xi^{(u)}}\right|_{1-\xi^{(u)}} ^{1+\xi^{(u)}} \\
& =1+\frac{\left(\xi^{(u)}\right)^{2}}{3}
\end{aligned}
$$

Taking $\xi^{(u)}=\sqrt{3} \xi^{(n)}=\sqrt{3} \xi^{(b i)}=\sqrt{3} \xi$

$$
\begin{aligned}
M_{2}^{(u)} & =1+\frac{\left(\xi^{(u))^{2}}\right.}{3}(\text { from equation (C.15)) } \\
& =1+\frac{\left(\sqrt{3} \xi^{(n)}\right)^{2}}{3} \text { (Replacement) } \\
& =1+\xi^{2},
\end{aligned}
$$

thereby, placing a limit on the uniform distribution $([1-\sqrt{3} \xi, 1+\sqrt{3} \xi])$ so that the intention of keeping the first two moments of three distribution is preserved.

From equations (C.4), (C.10) and (C.14), it can be said that the first moment of the distributions have been matched. From equations (C.7), (C.11) and after a placing a limit on the uniform distribution, equation(C.16) shows that the second moments of the distributions are matched. However, for large disorder, there is a need for correction as $b>0$ and cannot be negative. 



\section{Appendix D}

\section{Energy Equations in the Matrix Form}

Energy Equations (Kinetic, Potential and Total Energy) are used for analyzing the properties of mechanical wave propagation across the granular chain. The matrix form of the equations assists in computing the signals both in real and wavenumber space with ease. The equations (3.27),(3.28),(3.30) \& (3.31) (used in Chapter-3) are derived in this section using a three particles system $(i-1, i$ and $i+1)$. For better visualization, the pre-compressed granular chain is represented by a spring mass system as shown in Fig. D.1. The springs are the interactions between the elements, resembling particle contacts.

Kinetic Energy During the initial pre-compressed static, equilibrium state, the elements/particles are not in motion, hence their respective velocities are 0 , therefore, the Kinetic Energy is 0. The Kinetic Energy associated with particle $p$ during wave propagation is given by

$$
K E^{(p)}(t)=\frac{1}{2} b^{(p)}\left(v^{(p)}\right)^{2} .
$$


Initial pre-compressed state :

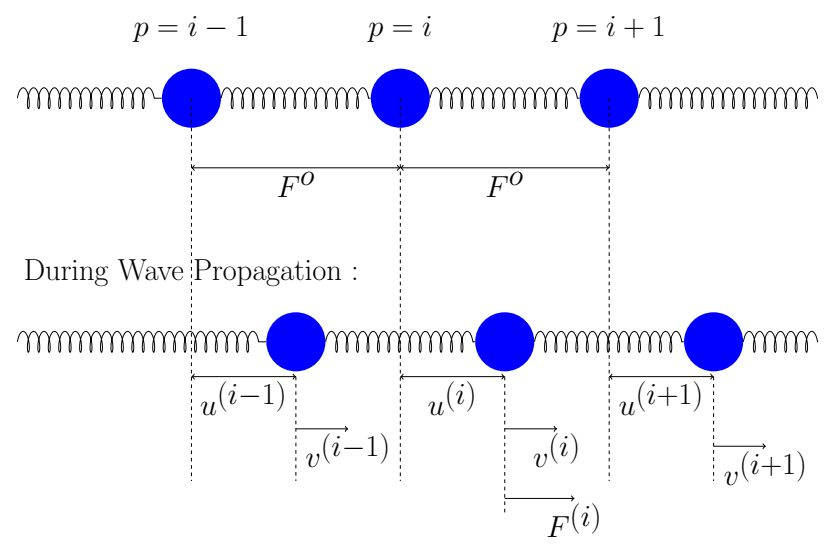

Figure D.1: Particle chain for simple test cases of energy equations.

The Kinetic Energy can also be calculated by the use of matrices as in Eq. (3.27),

$$
\begin{aligned}
& \mathbf{K E}=\frac{1}{2} \mathbf{M}[\mathbf{v} \otimes \mathbf{v}]=\frac{1}{2} \mathbf{M v v}^{\mathbf{T}} \\
& =\frac{1}{2}\left(\begin{array}{ccccc}
\ddots & \vdots & \vdots & \vdots & \vdots \\
\ldots & b^{(i-1)} & 0 & 0 & \ldots \\
\ldots & 0 & b^{(i)} & 0 & \ldots \\
\ldots & 0 & 0 & b^{(i+1)} & \ldots \\
\vdots & \vdots & \vdots & \vdots & \ddots
\end{array}\right)\left(\begin{array}{c}
\vdots \\
v^{(i-1)} \\
v^{(i)} \\
v^{(i+1)} \\
\vdots
\end{array}\right)\left(\begin{array}{lllll}
\ldots & v^{(i-1)} & v^{(i)} & v^{(i+1)} & \ldots
\end{array}\right) \\
& =\frac{1}{2}\left(\begin{array}{ccccc}
\ddots & \vdots & \vdots & \vdots & \vdots \\
\ldots & b^{(i-1)}\left(v^{(i-1)}\right)^{2} & b^{(i-1)} v^{(i-1)} v^{(i)} & b^{(i-1)} v^{(i-1)} v^{(i+1)} & \ldots \\
\ldots & b^{(i)} v^{(i-1)} v^{(i)} & b^{(i)}\left(v^{(i)}\right)^{2} & b^{(i)} v^{(i)} v^{(i+1)} & \ldots \\
\ldots & b^{(i+1)} v^{(i-1)} v^{(i+1)} & b^{(i+1)} v^{(i)} v^{(i+1)} & b^{(i+1)}\left(v^{(i+1)}\right)^{2} & \ldots \\
\vdots & \vdots & \vdots & \vdots & \ddots
\end{array}\right)
\end{aligned}
$$


The diagonal elements of this matrix give the Kinetic Energy of individual elements (Eq. (D.1)) and the Total Kinetic Energy of the system is the trace of this matrix. The non-diagonal elements give the spatial velocity correlation of the elements with other elements in the system. For instance, $K E_{12}$ is the velocity correlation of the 1st element with the 2 nd element.

Potential Energy The Potential energy during the initial precompression state is due to the initial static overlap. However, during wave propagation the potential energy is calculated as

$$
P E^{(p)}(t)=\left(P E_{(i-1, i)}+P E_{(i, i+1)}\right) / 2 ;
$$

where $P E^{(p)}(t)$ is the potential energy of the individual particles, $P E_{(i-1, i)}$ and $P E_{(i, i+1)}$ are potential energies due to the adjacent springs (contacts). According to the definition of Potential Energy,

$$
P E_{(i, j)}=-\int\left(F_{(i, j)}-F_{o}\right) d x,
$$

where $F_{o}$ is the force between particles during initial static overlap, $F_{(i, j)}$ is the dynamic force between particles $i$ and $j$ and $x$ is the change in the length of the spring. Hence, using Hertzian contact forces (Eq. (3.5))

$$
\begin{aligned}
P E_{(i, j)} & =-\int_{\Delta_{(i, j)}}^{\Delta_{(i, j)}-u^{(i)}+u^{(j)}}\left[\kappa_{(i, j)}\left(\Delta_{(i, j)}-u^{(i)}+u^{(j)}\right)^{3 / 2}-\kappa_{(i, j)} \Delta_{(i, j)}^{3 / 2}\right] \mathrm{d} x, \\
& =\frac{2}{5} \kappa_{(i, j)}\left[\Delta_{(i, j)}-u^{(i)}+u^{(j)}\right]^{5 / 2}-\frac{2}{5} \kappa_{(i, j)} \Delta_{i, j}^{5 / 2}-\kappa_{(i, j)} \Delta_{i, j}^{3 / 2}\left(u^{(j)}-u^{(i)}\right), \\
& =\frac{2}{5} \kappa_{(i, j)} \Delta_{(i, j)}^{5 / 2}\left[1+\frac{\left(u^{(j)}-u^{(i)}\right)}{\Delta_{(i, j)}}\right]^{5 / 2}-\frac{2}{5} \kappa_{(i, j)} \Delta_{i, j}^{5 / 2}-\kappa_{(i, j)} \Delta_{(i, j)}^{3 / 2}\left(u^{(j)}-u^{(i)}\right), \\
& \left(\text { Using Newton's expansion, }(1+x)^{m}=1+m x+m(m-1) \frac{x^{2}}{2 !}+\ldots\right) \\
& (\text { Neglecting higher order and lower coefficient terms }) \\
& \cong \frac{3}{4} \kappa_{(i, j)}^{2 / 3}\left(\left(u^{(i)}\right)^{2}-u^{(i)} u^{(i+1)}\right) .
\end{aligned}
$$


Hence, using Eq. (D.3)

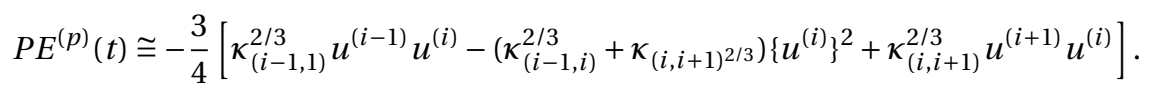

The Potential Energy in the matrix form is computed as (Eq. (3.30))

$$
\begin{aligned}
& \mathbf{P E}=-\frac{1}{2} \mathbf{K}[\mathbf{u} \otimes \mathbf{u}]=\frac{1}{2} \mathbf{K u \mathbf { u } ^ { \mathrm { T } }}, \\
& =-\frac{1}{2}\left(\begin{array}{ccccc}
\ddots & \vdots & \vdots & \vdots & \vdots \\
\ldots & -\frac{3}{2}\left(\kappa_{(i-2, i-1)}^{2 / 3}+\kappa_{(i-1, i)}^{2 / 3}\right) & \frac{3}{2} \kappa_{(i-1, i)}^{2 / 3} & 0 & \ldots \\
\cdots & \frac{3}{2} \kappa_{(i-1, i)}^{2 / 3} & -\frac{3}{2}\left(\kappa_{(i-1, i)}^{2 / 3}+\kappa_{(i, i+1)}^{2 / 3}\right) & \frac{3}{2} \kappa_{(i, i+1)}^{2 / 3} & \ldots \\
\cdots & 0 & \frac{3}{2} \kappa_{(i, i+1)}^{2 / 3} & -\frac{3}{2}\left(\kappa_{(i, i+1)}^{2 / 3}+\kappa_{(i+1, i+2)}^{2 / 3}\right) & \ldots \\
\vdots & \vdots & \vdots & \vdots & \ddots
\end{array}\right) \\
& \left(\begin{array}{c}
\vdots \\
u^{(i-1)} \\
u^{(i)} \\
u^{(i+1)} \\
\vdots
\end{array}\right)\left(\begin{array}{lllll}
\ldots & u^{(i-1)} & u^{(i)} & u^{(i+1)} & \ldots
\end{array}\right)
\end{aligned}
$$

The diagonal elements of the matrix

$$
P E_{P P}=-\frac{3}{4}\left[\kappa_{(i-1,1)}^{2 / 3} u^{(i-1)} u^{(i)}-\left(\kappa_{(i-1, i)}^{2 / 3}+\kappa_{(i, i+1)}^{2 / 3}\right)\left\{u^{(i)}\right\}^{2}+\kappa_{(i, i+1)}^{2 / 3} u^{(i+1)} u^{(i)}\right]
$$

which is the same as Eq. (D.6). Similar to KE matrix, the non-diagonal elements of PE give the spatial displacement correlation of the elements with other elements in the system. 


\section{Bibliography}

[1] R. AbReU, J. KAMm, and A. ReIss. "Micropolar modelling of rotational waves in seismology". In: Geophysical Journal International 210 (Iss. 2 2017), pp. 1021-1046 (cit. on pp. 5, 8).

[2] V. Achilleos, G. Theocharis, and Ch. Skokos. "Energy transport in one-dimensional disordered granular solids". In: Phys. Rev. E 93 (Iss. 2 2016), p. 022903. DOI: 10.1103/PhysRevE. 93.022903 (cit. on p. 18).

[3] C. M. Aegerter, M. Störzer, and G. Maret. "Experimental determination of critical exponents in Anderson localisation of light". In: EPL (Europhysics Letters) 75.(4) (2006), p. 562 (cit. on p. 7).

[4] K. AKI and B. ChouEt. "Origin of coda waves: source, attenuation, and scattering effects". In: Journal of geophysical research 80.(23) (1975), pp. 3322-3342 (cit. on pp. 5, 7, 8, 11).

[5] K. AKI and P. G. RichaRds. Quantitative Seismology. Geology (University Science Books).: Seismology. University Science Books, 2002 (cit. on pp. 1, 16).

[6] M. P. van AlBADA and A. LAGENDIJK. "Observation of weak localization of light in a random medium". In: Physical review letters 55.(24) (1985), p. 2692 (cit. on p. 8).

[7] P. B. AlLen and J. Kelner. "Evolution of a vibrational wave packet on a disordered chain". In: American Journal of Physics 66.(6) (1998), pp. 497-506. DOI: http://dx. doi .org/10.1119/1 . 18890 (cit. on pp. $26,60,65)$. 
[8] G. Alvarado and M. R. Coop. "On the performance of bender elements in triaxial tests". In: Géotechnique 62.(1) (2012), pp. 1-17. DOI: 10 . 1680/geot. 7.00086 (cit. on p. 6).

[9] P. W. Anderson. "Absence of Diffusion in Certain Random Lattices". In: Phys. Rev. 109 (Iss. 5 1958), pp. 1492-1505. DOI: 10.1103/PhysRev . 109.1492 (cit. on pp. 7, 26, 100).

[10] R. Arulnathan, R. W. Boulanger, and M. F. Riemer. "Analysis of bender element tests". In: Geotechnical Testing Journal 21.(2) (1998), pp. 120-131 (cit. on p. 6).

[11] J. H. ATKINSON. "Non-linear soil stiffness in routine design”. In: Géotechnique 50.(5) (2000), pp. 487-508 (cit. on p. 10).

[12] A. Aubry and A. Derode. "Multiple scattering of ultrasound in weakly inhomogeneous media: Application to human soft tissues". In: The Journal of the Acoustical Society of America 129.(1) (2011), pp. 225-233 (cit. on p. 2).

[13] M. YA. AzBeL. "One dimension. Thermodynamics, kinetics, eigenstates, and universality". In: Phys. Rev. B 27 (Iss. 6 1983), pp. 3901-3904. DOI: 10.1103/PhysRevB. 27.3901 (cit. on p. 40).

[14] M. VAN DER BAAN. "Acoustic wave propagation in one dimensional random media: the wave localization approach". In: Geophysical Journal International 145.(3) (2001), pp. 631-646. DOI: 10 . $1046 / \mathrm{j} .1365$ 246x.2001.01405.x (cit. on p. 16).

[15] G. E. BACKUS. "Long-wave elastic anisotropy produced by horizontal layering". In: Journal of Geophysical Research 67.(11) (1962), pp. 44274440 (cit. on p. 8).

[16] G. Baldi, V. M. Giordano, G. Monaco, and B. Ruta. "Sound attenuation at terahertz frequencies and the boson peak of vitreous silica". In: Physical review letters 104.(19) (2010), p. 195501 (cit. on p. 78).

[17] G. Baldi, V. M. Giordano, B. Ruta, R. Dal Maschio, A. Fontana, and G. MonACO. "Anharmonic damping of terahertz acoustic waves in a network glass and its effect on the density of vibrational states". In: Physical review letters 112.(12) (2014), p. 125502 (cit. on p. 78).

[18] D. S. Bassett, E. T. Owens, K. E. Daniels, and M. A. Porter. "Influence of network topology on sound propagation in granular materials". In: Phys. Rev. E 86 (Iss. 4 2012), p. 041306. DOI: 10.1103/PhysRevE. 86.041306 (cit. on p. 10). 
[19] D. S. Bassett, E. T. Owens, M. A. Porter, M. L. Manning, and K. E. DANIELS. "Extraction of force-chain network architecture in granular materials using community detection". In: Soft Matter 11 (Iss. 14 2015), pp. 2731-2744. DOI: 10.1039/C4SM01821D (cit. on p. 10).

[20] G. BAYER and T. NIEDERDRÄNK. "Weak localization of acoustic waves in strongly scattering media”. In: Physical review letters 70.(25) (1993), p. 3884 (cit. on p. 8).

[21] R. P. Behringer, D. Howell, L. Kondic, S. Tennakoon, and C. Veje. "Predictability and granular materials". In: Physica D: Nonlinear Phenomena 133.(1) (1999), pp. 1 -17. DoI: https://doi.org/10.1016/ S0167-2789 (99)00094-9 (cit. on p. 10).

[22] R. J. BELL and P. DEAN. "Atomic vibrations in vitreous silica". In: Discuss. Faraday Soc. 50 (Iss. 0 1970), pp. 55-61. DOI: 10.1039/DF9705000055 (cit. on p. 26).

[23] J. Billy, V. Josse, Z. Zuo, A. Bernard, B. Hambrecht, P. Lugan, D. Clément, L. Sanchez-Palencia, P. Bouyer, and A. Aspect. "Direct observation of anderson localization of matter waves in a controlled disorder". In: Nature 453.(7197) (2008), p. 891 (cit. on p. 7).

[24] E. Bitzek, P. Koskinen, F. GÄhler, M. Moseler, and P. Gumbsch. "Structural relaxation made simple". In: Physical review letters 97.(17) (2006), p. 170201 (cit. on p. 80).

[25] N. Boechler, G. Theocharis, and C. Daraio. "Bifurcation-based acoustic switching and rectification". In: Nat Mater 10.(9) (2011), pp. 665668 (cit. on pp. 2, 17, 96).

[26] N. Boechler, G. Theocharis, S. Job, P. G. Kevrekidis, Mason A. PORTER, and C. DARAIO. "Discrete Breathers in One-Dimensional Diatomic Granular Crystals". In: Phys. Rev. Lett. 104 (Iss. 24 2010), p. 244302. DOI: 10.1103/PhysRevLett.104.244302 (cit. on p. 12).

[27] N. Boechler, J. YAng, G. Theocharis, P.G. KeVrekidis, and C. Daraio. "Tunable vibrational band gaps in one-dimensional diatomic granular crystals with three-particle unit cells". In: Journal of Applied Physics 109.(7) (2011), p. 074906. DOI: 10.1063/1. 3556455 (cit. on p. 12).

[28] L. BRILlouin. Wave propagation in periodic structures. Dover Phoenix Editions. McGraw-Hill Book Company, Inc., 1946 (cit. on p. 27). 
[29] T. BRunet, X. JiA, and P. Mills. "Mechanisms for acoustic absorption in dry and weakly wet granular media". In: Physical review letters 101.(13) (2008), p. 138001 (cit. on p. 92).

[30] A. A. Chabanov, M. Stoytchev, and A. Z. Genack. "Statistical signatures of photon localization". In: Nature 404.(6780) (2000), p. 850 (cit. on p. 7).

[31] A. A. Chabanov, Z. Q. Zhang, and A. Z. Genack. "Breakdown of diffusion in dynamics of extended waves in mesoscopic media". In: Physical review letters 90.(20) (2003), p. 203903 (cit. on p. 7).

[32] J. Chabé, G. Lemarié, B. Grémaud, D. Delande, P. Szriftgiser, and J. C. GARREAU. "Experimental observation of the Anderson metalinsulator transition with atomic matter waves". In: Physical review letters 101.(25) (2008), p. 255702 (cit. on p. 7).

[33] S. ChANDRASEKHAR. Radiative transfer. Courier Corporation, 2013 (cit. on p. 8).

[34] C. Chapman. Fundamentals of seismic wave propagation. Cambridge university press, 2004 (cit. on p. 1).

[35] D. C. Charmpis, G. I. SchuËLler, and M. F. Pellissetti. "The need for linking micromechanics of materials with stochastic finite elements: a challenge for materials science". In: Computational Materials Science 41.(1) (2007), pp. 27-37 (cit. on p. 11).

[36] L. A. Cobus, S. E. Skipetrov, A. Aubry, B. A. van Tiggelen, A. Derode, and J. H. PAge. "Anderson Mobility Gap Probed by Dynamic Coherent Backscattering". In: Phys. Rev. Lett. 116 (Iss. 19 2016), p. 193901. DOI: 10.1103/PhysRevLett.116.193901 (cit. on p. 8).

[37] Wikipedia CONTRibutors. Acoustics. [Online; accessed 5-January-2018]. 2018 (cit. on p. 1).

[38] Wikipedia contributors. Photoelasticity. [Online; accessed 29-May2018]. 2018 (cit. on p. 10).

[39] E. Cosserat, F. Cosserat, et al. Théorie des corps déformables. A. Hermann et fils, 1909 (cit. on pp. 5, 78).

[40] C. Coste and B. GiLLEs. "Sound propagation in a constrained lattice of beads: High-frequency behavior and dispersion relation". In: Phys. Rev. E 77 (Iss. 2 2008), p. 021302. DOI: 10 . 1103/PhysRevE. 77.021302 (cit. on p. 16). 
[41] T. Cuccovillo and M. R. Coop. "The measurement of local axial strains in triaxial tests using LVDTs". In: Géotechnique 47.(1) (1997), pp. 167171 (cit. on p. 10).

[42] P. A. Cundall and O. D. L. StRack. "A discrete numerical model for granular assemblies". In: Geotechnique 29.(1) (1979), pp. 47-65 (cit. on p. 9).

[43] A. M. Dainty and M. N. Toksöz. "Seismic codas on the Earth and the Moon: a comparison". In: Physics of the Earth and Planetary Interiors 26.(4) (1981), pp. 250 -260. DOI: https : //doi .org/10.1016/00319201 (81) 90029-7 (cit. on pp. 5, 94).

[44] R. Dalichaouch, J. P. Armstrong, S. Schultz, P. M. Platzman, and S. L. MCCALL. "Microwave localization by two-dimensional random scattering". In: Nature 354.(6348) (1991), p. 53 (cit. on p. 7).

[45] K. E. DANiELS and N. W. HAYMAN. "Force chains in seismogenic faults visualized with photoelastic granular shear experiments". In: Journal of Geophysical Research: Solid Earth 113.(B11) (2008) (cit. on p. 10).

[46] C. Daraio, V. F. Nesterenko, E. B. Herbold, and S. Jin. "Energy Trapping and Shock Disintegration in a Composite Granular Medium". In: Phys. Rev. Lett. 96 (Iss. 5 2006), p. 058002. DOI: 10.1103/PhysRevLett. 96.058002 (cit. on pp. 2, 17).

[47] G. DEODATIS. "Non-stationary stochastic vector processes: seismic ground motion applications". In: Probabilistic Engineering Mechanics 11.(3) (1996), pp. 149-167 (cit. on p. 11).

[48] P. J. DigBY. "The effective elastic moduli of porous granular rocks". In: Journal of Applied Mechanics 48.(4) (1981), pp. 803-808 (cit. on p. 8).

[49] R. Doney and S. SEN. "Decorated, Tapered, and Highly Nonlinear Granular Chain”. In: Phys. Rev. Lett. 97 (Iss. 15 2006), p. 155502. DOI: 10 . 1103/PhysRevLett.97.155502 (cit. on p. 2).

[50] R. L. Doney, J. H. Agui, and S. SEN. "Energy partitioning and impulse dispersion in the decorated, tapered, strongly nonlinear granular alignment: A system with many potential applications". In: Journal of applied physics 106.(6) (2009), p. 064905. DOI: 10.1063/1.3190485 (cit. on p. 12). 
[51] J. DufFy and R. D. Minduin. Stress-strain relations and vibrations of a granular medium. Tech. rep. COLUMBIA UNIV NEW YORK DEPT OF CIVIL ENGINEERING and ENGINEERING MECHANICS, 1956 (cit. on p. 8).

[52] A. V. Dyskin, E. Pasternak, and E. Pelinovsky. "Periodic motions and resonances of impact oscillators". In: Journal of Sound and Vibration 331.(12) (2012), pp. 2856 -2873. DoI: https://doi.org/10.1016/j . jsv.2012.01.031 (cit. on p. 18).

[53] A. V. Dyskin, E. PASTERnAK, and I. SHUfRIN. "Structure of resonances and formation of stationary points in symmetrical chains of bilinear oscillators". In: Journal of Sound and Vibration 333.(24) (2014), pp. 6590 -6606. DOI: https://doi.org/10.1016/j.jsv.2014.06.044 (cit. on p. 18).

[54] R. DYviK and C. MADShUs. "Lab Measurements of $G_{\max }$ Using Bender Elements". In: Advances in the art of testing soils under cyclic conditions. ASCE. 1985, pp. 186-196 (cit. on p. 10).

[55] A. S. ELNASHAI and L. Di SARNO. Fundamentals of earthquake engineering. Wiley New York, 2008 (cit. on p. 9).

[56] M. Esin, E. Pasternak, and A. V. Dyskin. "Stability of 2D discrete mass-spring systems with negative stiffness springs". In: physica status solidi (b) 253.(7) (2016), pp. 1395-1409. DOI: $10.1002 / \mathrm{pssb}$. 201552763 (cit. on p. 18).

[57] E. A. DE F. BRAgançA, A. Rosas, and K. Lindenberg. "Binary collision approximation for multi-decorated granular chains". In: Physica A: Statistical Mechanics and its Applications 392.(24) (2013), pp. 6198 6205. DOI: https://doi.org/10.1016/j.physa.2013.07.076 (cit. on p. 12).

[58] S. FLACH and A. V. GORBACH. "Discrete breathers-advances in theory and applications". In: Physics Reports 467.(1-3) (2008), pp. 1-116 (cit. on p. 12).

[59] F. Fraternali, M. A. Porter, and C. Daraio. "Optimal design of composite granular protectors". In: Mechanics of Advanced Materials and Structures 17.(1) (2009), pp. 1-19. DOI: 10.1115/1. 3173118 (cit. on p. 2). 
[60] S. Gelin, H. TANAKA, and A. Lemaître. "Anomalous phonon scattering and elastic correlations in amorphous solids". In: Nature materials 15.(11) (2016), p. 1177 (cit. on pp. 78, 81, 85, 86).

[61] B. GiLles and C. Coste. "Low-Frequency Behavior of Beads Constrained on a Lattice". In: Phys. Rev. Lett. 90 (Iss. 17 2003), p. 174302. DOI: 10.1103/PhysRevLett.90.174302 (cit. on p. 16).

[62] J. D. GODDARD. "Nonlinear elasticity and pressure-dependent wave speeds in granular media". In: Proc. R. Soc. Lond. A 430.(1878) (1990), pp. 105131 (cit. on p. 12).

[63] L. R. Gómez, A. M. Turner, M. VAn Hecke, and V. Vitelli. "Shocks near jamming". In: Physical review letters 108.(5) (2012), p. 058001 (cit. on p. 78).

[64] L. R. Gómez, A. M. Turner, and V. Vitelli. "Uniform shock waves in disordered granular matter". In: Physical Review E 86.(4) (2012), p. 041302 (cit. on p. 78).

[65] P. D. GREENING and D. F. T. NASH. "Frequency domain determination of G 0 using bender elements". In: Geotechnical Testing Journal 27.(3) (2004), pp. 288-294 (cit. on p. 6).

[66] T. S. Grigera, V. Martin-Mayor, G. Parisi, and P. Verrocchio. "Phonon interpretation of the "boson peak'in supercooled liquids". In: Nature 422.(6929) (2003), p. 289 (cit. on p. 78).

[67] I. GÜVEN. "Hydraulical and acoustical properties of porous sintered glass bead systems: experiments, theory, \& simulations". PhD thesis. Enschede, 2016 (cit. on pp. 7, 16).

[68] I. GÜVEN, S. LUDING, and H. STEEB. "Incoherent waves in fluid-saturated sintered granular systems: Scattering phenomena". In: Journal of Vibration and Acoustics 140.(1) (2018), p. 011018 (cit. on p. 7).

[69] A. Guzek, A. V. Dyskin, E. Pasternak, and I. Shufrin. "Asymptotic analysis of bilinear oscillators with preload". In: International Journal of Engineering Science 106 (2016), pp. 125 -141. DoI: https: //doi .org/ 10.1016/j.ijengsci.2016.05.006 (cit. on p. 18).

[70] U. Harbola, A. Rosas, M. Esposito, and K. Lindenberg. "Pulse propagation in tapered granular chains: An analytic study". In: Phys. Rev. E 80 (Iss. 3 2009), p. 031303. DOI: 10.1103/PhysRevE. 80.031303 (cit. on p. 12). 
[71] J. Hong. "Universal Power-Law Decay of the Impulse Energy in Granular Protectors". In: Phys. Rev. Lett. 94 (Iss. 10 2005), p. 108001. DoI: 10.1103/PhysRevLett.94.108001 (cit. on p. 2).

[72] H. Hu, A. Strybulevych, J. H. Page, S. E. Skipetrov, and B. A. van TIGGELEN. "Localization of ultrasound in a three-dimensional elastic network". In: Nature Physics 4.(12) (2008), p. 945 (cit. on pp. 8, 10, 92).

[73] A. Ishimaru. Wave propagation and scattering in random media. Vol. 2. Academic press New York, 1978 (cit. on p. 8).

[74] G. James, P. G. Kevrekidis, and J. Cuevas. "Breathers in oscillator chains with Hertzian interactions". In: Physica D: Nonlinear Phenomena 251 (2013), pp. 39-59 (cit. on p. 12).

[75] P. JeHEL. "A Stochastic Multi-scale Approach for Numerical Modeling of Complex Materials-Application to Uniaxial Cyclic Response of Concrete". In: Computational Methods for Solids and Fluids. Springer, 2016, pp. 123-160 (cit. on p. 11).

[76] X. JiA. "Coda like Multiple Scattering of Elastic Waves in Dense Granular Media". In: Phys. Rev. Lett. 93 (Iss. 15 2004), p. 154303. DOI: 10. 1103/PhysRevLett.93.154303 (cit. on pp. 5, 10, 32, 92, 94).

[77] X. Jia, C. CARoli, and B. Velicky. "Ultrasound Propagation in Externally Stressed Granular Media”. In: Phys. Rev. Lett. 82 (Iss. 9 1999), pp. 1863-1866. DOI: 10.1103/PhysRevLett . 82.1863 (cit. on pp. 5, $16,32,94)$.

[78] X. Jia, J. Laurent, Y. Khidas, and V. LANGlois. "Sound scattering in dense granular media". In: Chinese Science Bulletin 54.(23) (2009), pp. 4327-4336 (cit. on p. 10).

[79] D. L. Johnson, Y. Hu, and H. MAKSE. "Density of states in granular media in the presence of damping". In: Physical Review E 91.(6) (2015), p. 062208 (cit. on p. 92).

[80] P. A. Johnson and X. JiA. "Nonlinear dynamics, granular media and dynamic earthquake triggering". In: Nature 437.(7060) (2005), p. 871 (cit. on p. 10).

[81] V. Jovičić and M. R. Coop. "Stiffness of coarse-grained soils at small strains". In: Géotechnique 47.(3) (1997), pp. 545-561 (cit. on p. 6).

[82] N. G. VAN Kampen. Stochastic processes in physics and chemistry. Vol. 1. Elsevier, 1992 (cit. on p. 53). 
[83] W. R. KAmpinga, Y. H. WiJnant, and A. DE BoeR. "Viscothermal wave propagation in a circular layer with a partially open and partrally closed boundary". In: 2006 International Conference on Noise and Vibration Engineering, ISMA 2006. Katholieke Universiteit Leuven. 2006 (cit. on p. 11).

[84] J. KAWAHARA. "Scattering attenuation of elastic waves due to low-contrast inclusions". In: Wave Motion 48.(3) (2011), pp. 290 -300. DOI: https : //doi.org/10.1016/j.wavemoti.2010.11.004 (cit. on p. 3).

[85] P. Kearey, M. Brooks, and I. Hill. An introduction to geophysical exploration. John Wiley \& Sons, 2013 (cit. on p. 1).

[86] D. KHATRI, D. NGO, and C. DARAIO. "Highly nonlinear solitary waves in chains of cylindrical particles". In: Granular Matter 14.(1) (2012), pp. 63-69 (cit. on p. 11).

[87] E. Kim, A. J. Martínez, S. E. Phenisee, P. G. Kevrekidis, M. A. PORTER, and J. YANG. "Direct measurement of superdiffusive energy transport in disordered granular chains". In: Nature communications 9.(1) (2018), p. 640. DOI: $10.1038 /$ s41467-018-03015-3 (cit. on pp. 9, 12).

[88] L. KnOpoff. “Q”. In: Reviews of Geophysics 2.(4) (1964), pp. 625-660. DOI: 10.1029/RG002i004p00625 (cit. on p. 3).

[89] N. P. KRUYT. "Micromechanical study of fabric evolution in quasi-static deformation of granular materials". In: Mechanics of Materials 44 (2012). Microstructures and Anisotropies, pp. 120 -129. DoI: https : // doi . org/10.1016/j.mechmat.2011.07.008 (cit. on p. 9).

[90] N. P. KRUYT. "Three-dimensional lattice-based dispersion relations for granular materials". In: AIP Conference Proceedings. Vol. 1227. 1. AIP. 2010, pp. 405-415. DOI: 10.1063/1.3435410 (cit. on p. 9).

[91] L. D. LANDAU and EvgeniI MikHaǏLOVICH LifShitz. Theory of elasticity. Pergamon Press, 1970 (cit. on pp. 53, 55, 98, 119).

[92] V. LANGLOIS and X. JiA. "Acoustic probing of elastic behavior and damage in weakly cemented granular media". In: Physical Review E 89.(2) (2014), p. 023206 (cit. on p. 2).

[93] V. LANGLOIS and X. JiA. "Sound pulse broadening in stressed granular media". In: Phys. Rev. E 91 (Iss. 2 2015), p. 022205. DOI: 10.1103 / PhysRevE. 91.022205 (cit. on pp. 5, 43, 94, 95, 101, 102, 104, 106, 112). 
[94] E. LAROSE. "Mesoscopics of ultrasound and seismic waves: application to passive imaging". In: Annales de Physique. Vol. 31. 3. 2006, pp. 1-126 (cit. on p. 8).

[95] E. LARose, O. I. LobKis, and R. L. WeAVER. "Coherent backscattering of ultrasound without a source". In: EPL (Europhysics Letters) 76.(3) (2006), p. 422 (cit. on pp. 2, 8).

[96] E. Larose, L. Margerin, B. A. van Tiggelen, and M. Campillo. "Weak Localization of Seismic Waves". In: Phys. Rev. Lett. 93 (Iss. 4 2004), p. 048501. DOI: 10.1103 / PhysRevLett . 93.048501 (cit. on pp. 8, 16).

[97] B. P. LAWNEY and S. LUDING. "Frequency filtering in disordered granular chains". English. In: Acta Mechanica 225.(8) (2014), pp. 2385-2407. DOI: $10.1007 / \mathrm{s} 00707-014-1130-4$ (cit. on pp. 8, 11, 17-19, 24, 27, $30,39,42,52,58,119)$.

[98] B. P. LAWNEY and S. LUDING. "Mass-disorder effects on the frequency filtering in one-dimensional discrete particle systems". In: AIP Conference Proceedings 1542.(1) (2013), pp. 535-538. DoI: http://dx . doi . org/10.1063/1.4811986 (cit. on pp. 25, 30, 58, 96, 100).

[99] J. B. Legland, Y. Zhang, O. Abraham, O. Durand, and V. Tournat. "Evaluation of crack status in a meter-size concrete structure using the ultrasonic nonlinear coda wave interferometry". In: The Journal of the Acoustical Society of America 142.(4) (2017), pp. 2233-2241. DOI: 10. 1121/1.5007832 (cit. on p. 2).

[100] M. LEIBIG. "Model for the propagation of sound in granular materials". In: Phys. Rev. E 49 (Iss. 2 1994), pp. 1647-1656. DOI: $10.1103 /$ PhysRevE.49.1647 (cit. on p. 9).

[101] M. M. LEVEN. Photoelasticity: The Selected Scientific Papers of MM Frocht. Elsevier, 2013 (cit. on p. 10).

[102] C. H. LiU and S. R. NAGEL. "Sound and vibration in granular materials". In: Journal of Physics: Condensed Matter 6.(23A) (1994), A433 (cit. on p. 9).

[103] C. H. LiU and S. R. NAGEL. "Sound in sand". In: Phys. Rev. Lett. 68 (Iss. 15 1992), pp. 2301-2304. DOI: 10.1103/PhysRevLett . 68 . 2301 (cit. on p. 9). 
[104] Chu-Heng LiU and Sidney R. NAGEL. "Sound in a granular material: Disorder and nonlinearity". In: Phys. Rev. B 48 (Iss. 21 1993), pp. 1564615650. DOI: 10.1103/PhysRevB.48.15646 (cit. on p. 9).

[105] V. Lubchenko and P. G. Wolynes. "The origin of the boson peak and thermal conductivity plateau in low-temperature glasses". In: Proceedings of the National Academy of Sciences 100.(4) (2003), pp. 1515-1518 (cit. on p. 78).

[106] S. LUDING and O. MourAILLE. "Mechanic waves in sand: effect of polydispersity". In: PARTEC 2007, Congress on Particle Technology. Ed. by W. PEUKert and C. SChrEglmann. University of Erlangen-Nuremberg, Institute of Particle Technology, 2008 (cit. on p. 39).

[107] T. S. MAJMUDAR. Experimental studies of two-dimensional granular systems using grain-scale contact force measurements. 2006 (cit. on p. 10).

[108] T. S. MAJMudAR and R. P. BEHRINGER. "Contact force measurements and stress-induced anisotropy in granular materials". In: Nature 435.(7045) (2005), p. 1079. DOI: 10.1038/nature03805 (cit. on p. 10).

[109] T. S. Majmudar, M. Sperl, S. Luding, and R. P. Behringer. "Jamming Transition in Granular Systems". In: Phys. Rev. Lett. 98 (Iss. 5 2007), p. 058001. DOI: 10.1103 / PhysRevLett . 98.058001 (cit. on p. 10).

[110] H. A. Makse, N. Gland, D. L. Johnson, and L. Schwartz. "Granular packings: Nonlinear elasticity, sound propagation, and collective relaxation dynamics". In: Phys. Rev. E 70 (Iss. 6 2004), p. 061302. DOI: 10.1103/PhysRevE.70.061302 (cit. on pp. 9, 12, 17, 95).

[111] H. A. Makse, N. Gland, D. L. Johnson, and L. M. SchWartz. "Why Effective Medium Theory Fails in Granular Materials". In: Phys. Rev. Lett. 83 (Iss. 24 1999), pp. 5070-5073. DOI: 10.1103 / PhysRevLett . 83 . 5070 (cit. on p. 9).

[112] Y. Man, N. Boechler, G. Theocharis, P. G. KeVrekidis, and C. Daraio. "Defect modes in one-dimensional granular crystals". In: Phys. Rev. E 85 (Iss. 3 2012), p. 037601. DOI: 10.1103/PhysRevE. 85.037601 (cit. on p. 12). 
[113] M. MAnciu, S. SEn, and A. J. Hurd. "The propagation and backscattering of soliton-like pulses in a chain of quartz beads and related problems. (I). Propagation". In: Physica A: Statistical Mechanics and its Applications 274.(3) (1999), pp. 588 -606. DOI: https ://doi .org/10. 1016/S0378-4371 (99)00371-4 (cit. on p. 12).

[114] L. MARgERIN. "Introduction to radiative transfer of seismic waves". In: Seismic Earth: Array Analysis of Broadband Seismograms (2005), pp. 229-252 (cit. on p. 8).

[115] G. MARKetos and C. O'Sullivan. "A micromechanics-based analytical method for wave propagation through a granular material". In: Soil Dynamics and Earthquake Engineering 45 (2013), pp. 25 -34. DOI: http: //dx.doi.org/10.1016/j.soildyn.2012.10.003 (cit. on pp. 10, 18).

[116] A. Marruzzo, W. Schirmacher, A. Fratalocchi, and G. Ruocco. "Heterogeneous shear elasticity of glasses: the origin of the boson peak". In: Scientific reports 3 (2013), p. 1407 (cit. on p. 78).

[117] K. Matsuyama and H. Katsuragi. "Power law statistics of force and acoustic emission from a slowly penetrated granular bed". In: Nonlinear Processes in Geophysics 21.(1) (2014), pp. 1-8. DOI: 10.5194/npg-211-2014 (cit. on p. 16).

[118] G. R. McDowell and M. D. Bolton. "Micro mechanics of elastic soil". In: Soils and foundations 41.(6) (2001), pp. 147-152 (cit. on p. 12).

[119] A. MERKEL and S. LUDING. "Enhanced micropolar model for wave propagation in ordered granular materials". In: International Journal of Solids and Structures 106 (2017), pp. 91 -105. DoI: http://dx.doi .org/10. 1016/j.ijsolstr. 2016.11.029 (cit. on pp. 5, 78, 82, 83, 86-88, 92).

[120] A. Merkel, V. Tournat, and V. Gusev. "Dispersion of elastic waves in three-dimensional noncohesive granular phononic crystals: Properties of rotational modes". In: Phys. Rev. E 82 (Iss. 3 2010), p. 031305. DOI: 10.1103/PhysRevE. 82.031305 (cit. on pp. 17, 78, 92).

[121] A. Merkel, V. Tournat, and V. Gusev. "Experimental evidence of rotational elastic waves in granular phononic crystals". In: Physical Review Letters 107.(22) (2011), p. 225502 (cit. on pp. 5, 78).

[122] H. Mizuno, K. SAitoh, and L. E. Silbert. "Elastic moduli and vibrational modes in jammed particulate packings". In: Physical Review E 93.(6) (2016), p. 062905 (cit. on p. 80). 
[123] H. Mizuno, H. Shiba, and A. Ikeda. "Continuum limit of the vibrational properties of amorphous solids". In: Proceedings of the National Academy of Sciences (2017), p. 201709015 (cit. on p. 78).

[124] H. Mizuno and R. Yамамото. "General constitutive model for supercooled liquids: Anomalous transverse wave propagation". In: Physical review letters 110.(9) (2013), p. 095901 (cit. on p. 78).

[125] T. R. K. MoHAn and S. SEN. "Linearity stabilizes discrete breathers". In: Pramana 77.(5) (2011), pp. 975-986 (cit. on p. 12).

[126] G. Monaco and V. M. Giordano. "Breakdown of the Debye approximation for the acoustic modes with nanometric wavelengths in glasses". In: Proceedings of the national Academy of Sciences 106.(10) (2009), pp. 3659-3663 (cit. on p. 78).

[127] G. MonAco and S. Mossa. "Anomalous properties of the acoustic excitations in glasses on the mesoscopic length scale". In: Proceedings of the National Academy of Sciences 106.(40) (2009), pp. 16907-16912 (cit. on p. 78).

[128] O. Mouraille. "Sound propagation in dry granular materials : discrete element simulations, theory, and experiments". PhD thesis. Enschede, the Netherlands, 2009 (cit. on pp. 9, 11, 26).

[129] O. Mouraille and S. Luding. "Sound wave propagation in weakly polydisperse granular materials". In: Ultrasonics 48.(6-7) (2008). Selected Papers from \{ICU\} 2007, pp. 498 -505. DoI: http : / dx . doi . org/10.1016/j.ultras.2008.03.009 (cit. on pp. 27, 52).

[130] O. Mouraille, W. A. Mulder, and S. Luding. "Sound wave acceleration in granular materials". In: Journal of Statistical Mechanics: Theory and Experiment 2006.(07) (2006), P07023 (cit. on pp. 34, 42, 43, 106).

[131] H. B. MÜHLhaUs and I. VARDoulaKIs. "The thickness of shear bands in granular materials". In: Geotechnique 37.(3) (1987), pp. 271-283 (cit. on p. 5).

[132] T. Nakamura, H. TAKenaka, T. OKamoto, and Y. Kaneda. "FDM simulation of seismic-wave propagation for an aftershock of the $2009 \mathrm{Su}$ ruga Bay earthquake: effects of ocean-bottom topography and seawater layer". In: Bulletin of the Seismological Society of America 102.(6) (2012), pp. 2420-2435 (cit. on p. 11). 
[133] Y. NAKAMURA. "Seismic velocity structure of the lunar mantle". In: Journal of Geophysical Research: Solid Earth 88.(B1) (1983), pp. 677-686 (cit. on p. 5).

[134] V. E. NAZARov, A. V. Radostin, and V. Yu. ZaĬTSEV. "Self-demodulation of acoustic pulses in partially water-saturated river sand". In: Acoustical Physics 52.(3) (2006), pp. 301-306 (cit. on p. 12).

[135] V. Nesterenko. Dynamics of heterogeneous materials. Springer Science \& Business Media, 2013 (cit. on p. 12).

[136] V. F. NEsTERENKO. "Propagation of nonlinear compression pulses in granular media". In: J. Appl. Mech. Tech. Phys. 24 (Iss. 5 1983), pp. 733743 (cit. on pp. 12, 17, 24, 78, 95).

[137] V. F. Nesterenko. "Shock (Blast) Mitigation by "Soft" Condensed Matter". In: Symposium MM - Granular Material-Based Technologies. Vol. 759. MRS Proceedings. 2002. DOI: 10.1557/PROC-759-MM4. 3 (cit. on p. 2).

[138] P. H. F. Nicholson, P. Mollanen, T. KÄRKKÄInen, J. Timonen, and S. CHENG. "Guided ultrasonic waves in long bones: modelling, experiment and in vivo application". In: Physiological Measurement 23.(4) (2002), p. 755 (cit. on p. 2).

[139] B. K. Novikov, O. V. Rudenko, V. I. Timoshenko, and M. A. BReazeale. Nonlinear Underwater Acoustics by BK Novikov, OV Rudenko, and VI Timoshenko. 1988 (cit. on p. 12).

[140] J. O'DONOVAN. "Micromechanics of wave propagation through granular material". PhD thesis. Imperial College London, 2013 (cit. on pp. 9, 10, 12).

[141] J. O'Donovan, E. Ibraim, C. O'Sullivan, S. Hamlin, D. Muir Wood, and G. MARKETOS. "Micromechanics of seismic wave propagation in granular materials". In: Granular Matter 18.(3) (2016), p. 56. DOI: 10. 1007/s10035-015-0599-4 (cit. on pp. 9-11, 16, 94).

[142] J. O'Donovan, G. Marketos, and C. O'Sullivan. "Novel methods of bender element test analysis". English. In: Geomechanics from Micro to Macro 1 (2014), pp. 311-316 (cit. on pp. 1, 10).

[143] J. O'Donovan, C. O'Sullivan, G. Marketos, and D. Muir Wood. "Analysis of bender element test interpretation using the discrete element method". In: Granular Matter 17.(2) (2015), pp. 197-216. DOI: 10.1007/s10035-015-0552-6 (cit. on pp. 6, 9, 10, 39, 43, 101). 
[144] V. OGARKO and S. Luding. "Prediction of polydisperse hard-sphere mixture behavior using tridisperse systems". In: Soft Matter 9 (Iss. 40 2013), pp. 9530-9534. DOI: 10.1039/C3SM50964H (cit. on p. 32).

[145] S. Ostojic, E. Somfai, and B. Nienhuis. "Scale invariance and universality of force networks in static granular matter". In: Nature 439.(7078) (2006), pp. 828-830 (cit. on p. 17).

[146] E. T. OWENS and K. E. DANIELS. "Sound propagation and force chains in granular materials". In: EPL (Europhysics Letters) 94.(5) (2011), p. 54005 (cit. on pp. 9, 10, 17, 96).

[147] J. H. PAGE. "Ultrasonic wave transport in strongly scattering media". In: Nano Optics and Atomics: Transport of Light and Matter Waves, DS Wiersma, R. Kaiser, and L. Fallani, eds., IOS Press, Amsterdam, The Netherlands (2011), pp. 75-93 (cit. on pp. 7, 9).

[148] J. H. Page, I. P. Jones, H. P. Schriemer, M. L. Cowan, P. Sheng, and D. A. WEITZ. "Diffusive transport of acoustic waves in strongly scattering media". In: Physica B: Condensed Matter 263-264 (1999), pp. 37 39. DOI: https ://doi.org/10.1016/S0921-4526(98)01191-0 (cit. on pp. 5, 7).

[149] E. Pasternak, A. V. Dyskin, M. Esin, G. M. Hassan, and C. MacNish. "Rotations and pattern formation in granular materials under loading". In: Philosophical Magazine 95.(28-30) (2015), pp. 3122-3145 (cit. on pp. 5, 17).

[150] E. Pasternak, A. V. Dyskin, and G. Sevel. "Chains of oscillators with negative stiffness elements". In: Journal of Sound and Vibration 333.(24) (2014), pp. 6676 -6687. DoI: https : //doi .org/10.1016/j.jsv . 2014.06 .045 (cit. on p. 18).

[151] E. PAsternAK and H.-B. MÜHLhaus. "Generalised homogenisation procedures for granular materials". In: Journal of Engineering Mathematics 52.(1) (2005), pp. 199-229. DOI: 10.1007 /s10665-004-3950-z (cit. on p. 17).

[152] H. Pichard, A. Duclos, J-P. Groby, V. Tournat, and V. E. Gusev. "Localized transversal-rotational modes in linear chains of equal masses". In: Physical Review E 89.(1) (2014), p. 013201 (cit. on p. 92).

[153] J. G. Pucketт. State variables in granular materials: An investigation of volume and stress fluctuations. North Carolina State University, 2012 (cit. on p. 10). 
[154] J. G. Puckett and K. E. DANiEls. "Equilibrating Temperaturelike Variables in Jammed Granular Subsystems". In: Phys. Rev. Lett. 110 (Iss. 5 2013), p. 058001. DOI: 10.1103/PhysRevLett. 110.058001 (cit. on p. 10).

[155] J. G. Puckett, F. Lechenault, K. E. Daniels, and J-L. Thiffeault. "Trajectory entanglement in dense granular materials". In: Journal of Statistical Mechanics: Theory and Experiment 2012.(06) (2012), P06008 (cit. on p. 10).

[156] J. W. S. B. Rayleigh. The Theory of Sound. The Theory of Sound v. 1. Macmillan, 1877 (cit. on p. 1).

[157] G. Roati, C. D’Errico, L. FAllani, M. FATtori, C. Fort, M. Zaccanti, G. Modugno, M. Modugno, and M. Inguscio. "Anderson localization of a non-interacting bose-einstein condensate". In: Nature 453.(7197) (2008), p. 895 (cit. on p. 7).

[158] A. Rosas and K. Lindenberg. "Pulse propagation in granular chains". In: Physics Reports (2018). DOI: https : / / doi . org / 10 . 1016 / j . physrep.2018.02.001 (cit. on p. 12).

[159] W. Ru-SHAN. "Multiple scattering and energy transfer of seismic waves - separation of scattering effect from intrinsic attenuation - I. Theoretical modelling". In: Geophysical Journal of the Royal Astronomical Society 82.(1) (1985), pp. 57-80. DOI: 10.1111/j.1365-246X.1985.tb05128. $\mathrm{x}$ (cit. on p. 3).

[160] L. RyzhiK, G. Papanicolaou, and J. B. Keller. "Transport equations for elastic and other waves in random media". In: Wave motion 24.(4) (1996), pp. 327-370 (cit. on p. 8).

[161] W. SACHSE and Y-H PAO. "On the determination of phase and group velocities of dispersive waves in solids". In: Journal of applied Physics 49.(8) (1978), pp. 4320-4327 (cit. on p. 2).

[162] K. Saitoh, V. Magnanimo, and S. Luding. "A Master equation for the probability distribution functions of forces in soft particle packings". In: Soft matter 11.(7) (2015), pp. 1253-1258 (cit. on p. 82).

[163] KuniYasu Saitoh, Rohit K Shrivastava, and Stefan Luding. "Rotational sound in disordered granular materials". In: arXiv preprint 1807 (2018), p. 08201 (cit. on p. 164). 
[164] J. C. SAntamarina and M. Aloufi. "Small strain stiffness: A micromechanical experimental study". In: Proceedings of Pre-failure Deformation Characteristics of Geomaterials (1999), pp. 451-458 (cit. on p. 10).

[165] H. SATo. "Seismic Waves in Heterogeneous Earth, Scattering of". In: Extreme Environmental Events: Complexity in Forecasting and Early Warning. Ed. by Robert A. MeYers. Springer New York, New York, NY, 2011, pp. 788-804. DOI: $10.1007 / 978-1-4419-7695-6 \_42$ (cit. on p. 16).

[166] H. Sato, Michael C Fehler, and Takuto Maeda. Seismic wave propagation and scattering in the heterogeneous earth. Vol. 496. Springer, 2012 (cit. on p. 78).

[167] J. A. SCAles and E. S. VAN Vleck. "Lyapunov Exponents and Localization in Randomly Layered Media". In: J. Comput. Phys. 133.(1) (1997), pp. 27-42. DOI: 10.1006/jcph.1997. 5643 (cit. on p. 16).

[168] W. Schirmacher, G. Ruocco, and T. Scopigno. "Acoustic attenuation in glasses and its relation with the boson peak". In: Physical review letters 98.(2) (2007), p. 025501 (cit. on p. 78).

[169] C. F. Schreck, C. S. O'Hern, and M. D. Shattuck. "Vibrations of jammed disk packings with Hertzian interactions". In: Granular Matter 16.(2) (2014), pp. 209-216. DOI: 10.1007/s10035-013-0458-0 (cit. on p. 41).

[170] L. M. SchWARTZ, D. L. Johnson, and S. Feng. "Vibrational modes in granular materials". In: Physical review letters 52.(10) (1984), p. 831 (cit. on pp. 5, 9, 78).

[171] M. Segev, Y. Silberberg, and D. N. Christodoulides. "Anderson localization of light". In: Nature Photonics 7.(3) (2013), p. 197 (cit. on p. 7).

[172] S. SEN and T. R. K. MoHAN. "Dynamics of metastable breathers in nonlinear chains in acoustic vacuum". In: Physical Review E 79.(3) (2009), p. 036603 (cit. on p. 12).

[173] S. SEN and R. S. Sinkovits. "Sound propagation in impure granular columns". In: Phys. Rev. E 54 (Iss. 6 1996), pp. 6857-6865. DOI: 10 . 1103/PhysRevE. 54.6857 (cit. on p. 12).

[174] P. M. SHEARER. Introduction to seismology. Cambridge University Press, 2009 (cit. on pp. 1, 3, 6, 9, 94). 
[175] P. SHENG. Introduction to Wave Scattering, Localization and Mesoscopic Phenomena; 2nd ed. Springer, Dordrecht, 2006 (cit. on pp. 16, 78, 83, 92, 94, 100).

[176] D. J. ShiRley and L. D. HAMPton. "Shear wave measurements in laboratory sediments". English. In: The Journal of the Acoustical Society of America 63.(2) (1978), pp. 607-613. DOI: http://dx . doi .org/10 . 1121/1. 381760 (cit. on pp. 1, 10).

[177] R. K. ShrivastaVA and S. LUDING. "Effect of disorder on bulk sound wave speed: a multiscale spectral analysis". In: Nonlinear Processes in Geophysics 24.(3) (2017), pp. 435-454. DOI: 10 . 5194/npg-24-4352017 (cit. on pp. 13, 58, 65, 95, 96, 98, 100, 101, 106, 164).

[178] R. K. SHRIVASTAVA and S. LUDING. "Wave propagation of spectral energy content in a granular chain". In: EPJ Web of Conferences. Vol. 140. EDP Sciences. 2017, p. 02023. DOI: 10 . 1051/epjconf / 201714002023 (cit. on pp. 13, 58, 164).

[179] R. K. Shrivastava, A. Thomas, N. VRiend, and S. Luding. "Long Wavelength Coherent Pulse of Sound Propagating in Granular Media". In: World Academy of Science, Engineering and Technology, International Journal of Mechanical, Aerospace, Industrial, Mechatronic and Manufacturing Engineering 11.(10) (2017), pp. 1694-1701 (cit. on pp. 13, 164).

[180] L. E. Silbert, A. J. LiU, and S. R. NAGEL. "Normal modes in model jammed systems in three dimensions". In: Physical Review E 79.(2) (2009), p. 021308 (cit. on p. 78).

[181] L. E. Silbert, A. J. LiU, and S. R. NAGEL. "Vibrations and diverging length scales near the unjamming transition". In: Physical review letters 95.(9) (2005), p. 098301 (cit. on p. 78).

[182] J. SLUYs. Wave propagation, localisation and dispersion in softening solids (PhD Thesis). 1992 (cit. on p. 5).

[183] R. SNIEDER. "The Theory of Coda Wave Interferometry". In: pure and applied geophysics 163.(2) (2006), pp. 455-473. DOI: 10.1007/s00024005-0026-6 (cit. on p. 2).

[184] R. Snieder, A. Grêt, H. Douma, and J. Scales. "Coda Wave Interferometry for Estimating Nonlinear Behavior in Seismic Velocity". In: Science 295.(5563) (2002), pp. 2253-2255. DOI: 10.1126/science. 1070015 (cit. on pp. 7, 16). 
[185] E. Somfai, M. van Hecke, W. G. Ellenbroek, K. Shundyak, and W. VAN SAARLOOS. "Critical and noncritical jamming of frictional grains". In: Physical Review E 75.(2) (2007), p. 020301 (cit. on p. 92).

[186] E. Somfai, J-N. Roux, J. H. SnoeiJer, M. van Hecke, and W. van SAARLOOS. "Elastic wave propagation in confined granular systems". In: Phys. Rev. E 72 (Iss. 2 2005), p. 021301. DOI: 10.1103/PhysRevE. 72.021301 (cit. on pp. 10, 12, 17).

[187] B. Z. SPIVAK and D. E. KHMELNITSKII. "Influence of localization effects in a normal metal on the properties of SNS junction". In: JETP Lett 35.(8) (1982), pp. 412-416 (cit. on p. 8).

[188] M. Störzer, P. Gross, C. M. Aegerter, and G. Maret. "Observation of the critical regime near Anderson localization of light". In: Physical Review Letters 96.(6) (2006), p. 063904 (cit. on p. 7).

[189] A. SUIKER. The mechanical behaviour of ballasted railway tracks ( $\mathrm{PhD}$ Thesis). 2004 (cit. on p. 5).

[190] A. S. J. Suiker, C. S. ChAnG, R. DE Borst, and C. Esveld. "Surface waves in a stratified half space with enhanced continuum properties. Part 1: Formulation of the boundary value problem". In: European Journal of Mechanics-A/Solids 18.(5) (1999), pp. 749-768 (cit. on p. 5).

[191] A. S. J. Suiker, C. S. Chang, R. DE Borst, and C. Esveld. "Surface waves in a stratified half space with enhanced continuum properties. Part 2: Analysis of the wave characteristics in regard to high-speed railway tracks". In: European Journal of Mechanics-A/Solids 18.(5) (1999), pp. 769-784 (cit. on p. 5).

[192] D. SUN and S. SEN. "Nonlinear grain-grain forces and the width of the solitary wave in granular chains: a numerical study". In: Granular Matter 15.(2) (2013), pp. 157-161. DOI: 10.1007/s10035-013-0400-5 (cit. on p. 12).

[193] T. L. SzABo and J. Wu. "A model for longitudinal and shear wave propagation in viscoelastic media". In: The Journal of the Acoustical Society of America 107.(5) (2000), pp. 2437-2446 (cit. on p. 3).

[194] K. Taghizadeh, S. Luding, and V. Magnanimo. "DEM applied to soil mechanics". In: ALERT Doctoral School 2017 Discrete Element Modeling (2017), p. 129 (cit. on p. 11). 
[195] Q. TAI and M. H. SADD. "A discrete element study of the relationship of fabric to wave propagational behaviours in granular materials". In: International Journal for Numerical and Analytical Methods in Geomechanics 21.(5) (1997), pp. 295-311. DOI: 10 .1002/ (SICI) 1096 9853 (199705) $21: 5<295$ : : AID-NAG871>3.0.C0;2-Y (cit. on p. 9).

[196] C. TIAN and A. LARKIN. "Deterministic Weak Localization in Periodic Structures”. In: Phys. Rev. Lett. 95 (Iss. 24 2005), p. 246601. DOI: 10 . 1103/PhysRevLett.95.246601 (cit. on p. 8).

[197] A. M. Tichler, L. R. Gomez, N. Upadhyaya, X. Campman, V. F. Nesterenko, and V. VITELLI. "Transmission and reflection of strongly nonlinear solitary waves at granular interfaces". In: Physical review letters 111.(4) (2013), p. 048001 (cit. on p. 78).

[198] R. TONN. "Comparison of seven methods for the computation of Q". In: Physics of the Earth and Planetary Interiors 55.(3) (1989), pp. 259-268. DOI: https : //doi .org/10 .1016/0031-9201(89) 90074-5 (cit. on p. 3).

[199] R. Tonn. "The determination of the seismic quality factor Q from VSP data: A comparison of different computational methods". In: Geophysical Prospecting 39.(1) (1991), pp. 1-27. DOI: 10.1111/j .1365-2478. 1991. tb00298.x (cit. on p. 3).

[200] A. Tourin, A. Derode, P. Roux, B. A. VAn Tiggelen, and M. Fink. "Time-dependent coherent backscattering of acoustic waves". In: Physical review letters 79.(19) (1997), p. 3637 (cit. on p. 8).

[201] V. Tournat, B. CAstagnède, V. Gusev, and P. BÉQuin. "Self-demodulation acoustic signatures for nonlinear propagation in glass beads". In: Comptes Rendus Mecanique 331.(2) (2003), pp. 119-125 (cit. on p. 12).

[202] V. Tournat and V. E. Gusev. "Acoustics of unconsolidated "model" granular media: An overview of recent results and several open problems". In: Acta Acustica united with Acustica 96.(2) (2010), pp. 208-224 (cit. on pp. 12, 17).

[203] V. Tournat, V. E. Gusev, and B. Castagnède. "Self-demodulation of elastic waves in a one-dimensional granular chain". In: Phys. Rev. E 70 (Iss. 5 2004), p. 056603. DOI: 10.1103/PhysRevE. 70.056603 (cit. on pp. 27, 65). 
[204] L. Trujillo, F. Peniche, and X. JiA. "Multiple scattering of elastic waves in granular media: Theory and experiments". In: Waves in Fluids and Solids. InTech, 2011 (cit. on p. 7).

[205] S. Ulrich, N. Upadhyaya, B. van Opheusden, and V. Vitelli. "Shear shocks in fragile networks". In: Proceedings of the National Academy of Sciences 110.(52) (2013), pp. 20929-20934 (cit. on p. 78).

[206] C. P. Umbach, C. VAn Haesendonck, R. B. Laibowitz, S. WashBURN, and R. A. WEBB. "Direct observation of ensemble averaging of the Aharonov-Bohm effect in normal-metal loops". In: Phys. Rev. Lett. 56 (Iss. 4 1986), pp. 386-389. DOI: 10.1103/PhysRevLett . 56.386 (cit. on p. 8).

[207] N. Upadhyaya, L. R. Gómez, and V. Vitelli. "Soliton Attenuation and Emergent Hydrodynamics in Fragile Matter". In: Phys. Rev. X 4 (Iss. 1 2014), p. 011045. DOI: $10.1103 /$ PhysRevX . 4.011045 (cit. on pp. 17, 78).

[208] B. VelickÝ and C. CAROLI. "Pressure dependence of the sound velocity in a two-dimensional lattice of Hertz-Mindlin balls: Mean-field description". In: Phys. Rev. E 65 (Iss. 2 2002), p. 021307. DOI: 10.1103 / PhysRevE.65.021307 (cit. on p. 9).

[209] F. O. WALKER. "Neuromuscular ultrasound". In: Neurologic clinics 22.(3) (2004), pp. 563-590 (cit. on p. 2).

[210] R. L. WEAVER. "Diffusivity of ultrasound in polycrystals". In: Journal of the Mechanics and Physics of Solids 38.(1) (1990), pp. 55-86 (cit. on p. 8).

[211] R. L. WEAVER. "Information from Seismic Noise". In: Science 307.(5715) (2005), pp. 1568-1569. DOI: 10.1126/science.1109834 (cit. on pp. 6, $16,52)$.

[212] R. L. WEAVER and W. SACHSE. "Diffusion of ultrasound in a glass bead slurry". In: The Journal of the Acoustical Society of America 97.(4) (1995), pp. 2094-2102. DOI: 10.1121/1.412002 (cit. on p. 7).

[213] D. S. Wiersma, P. BARTolini, A. LAgEndiJK, and R. Righini. "Localization of light in a disordered medium". In: Nature 390.(6661) (1997), p. 671 (cit. on p. 7).

[214] S. VAn den Wildenberg, M. VAn Hecke, and X. Jia. "Evolution of granular packings by nonlinear acoustic waves". In: EPL (Europhysics Letters) 101.(1) (2013), p. 14004 (cit. on pp. 5, 17). 
[215] S. VAn Den Wildenberg, A. Tourin, and X. JiA. "Sound velocity fluctuations in confined granular materials: Coarse-graining lengths and elastic heterogeneities". In: EPL 115.(3) (2016), p. 34005. DOI: 10 . 1209/0295-5075/115/34005 (cit. on pp. 1, 5, 96).

[216] S. VAn Den Wildenberg, Y. YAnG, and X. JiA. "Probing the effect of particle shape on the rigidity of jammed granular solids with sound speed measurements". In: Granular Matter 17.(4) (2015), pp. 419-426 (cit. on p. 10).

[217] P-E. Wolf and G. MARET. "Weak localization and coherent backscattering of photons in disordered media". In: Physical review letters 55.(24) (1985), p. 2696 (cit. on p. 8).

[218] S. WU, K. AKI, R-S. WU, and K. AKI. Scattering and attenuation of seismic waves. 1989 (cit. on p. 3).

[219] M. Wyart, S. R. NAgel, and T. A. Witten. "Geometric origin of excess low-frequency vibrational modes in weakly connected amorphous solids". In: EPL (Europhysics Letters) 72.(3) (2005), p. 486 (cit. on p. 78).

[220] M. Wyart, L. E. Silbert, S. R. Nagel, and T. A. Witten. "Effects of compression on the vibrational modes of marginally jammed solids". In: Physical Review E 72.(5) (2005), p. 051306 (cit. on p. 78).

[221] S. Yamashita, T. KaWAguchi, Y. Nakata, T. Mikami, T. FujiWara, and S. SHIBUYA. "Interpretation of international parallel test on the measurement of Gmax using bender elements". In: Soils and foundations 49.(4) (2009), pp. 631-650 (cit. on p. 6).

[222] J. YANG and X. GU. "Shear stiffness of granular material at small strains: does it depend on grain size?" In: Géotechnique (2013) (cit. on p. 11).

[223] J. YANG and M. SUTTON. "Nonlinear wave propagation in a hexagonally packed granular channel under rotational dynamics". In: International Journal of Solids and Structures 77 (2015), pp. 65 -73. DoI: http: // dx.doi.org/10.1016/j.ijsolstr.2015.07.017 (cit. on p. 17).

[224] Ö. YILmaz and S.M. DoherTy. Seismic Data Analysis: Processing, Inversion, and Interpretation of Seismic Data. Investigations in geophysics v. 1. Society of Exploration Geophysicists, 2001 (cit. on p. 1).

[225] Z. Zeravcic, N. Xu, A. J. LiU, S. R. NAgel, and W. van SAarloos. "Excitations of ellipsoid packings near jamming". In: EPL (Europhysics Letters) 87.(2) (2009), p. 26001 (cit. on p. 26). 
[226] Y. ZHu, A. SHukla, and M. H. SAdD. "The effect of microstructural fabric on dynamic load transfer in two dimensional assemblies of elliptical particles". In: Journal of the Mechanics and Physics of Solids 44.(8) (1996), pp. 1283 -1303. DOI: https : // doi . org/10 . 1016/0022 5096 (96)00036-1 (cit. on p. 10).

[227] V. A. ZvEREV. "How the idea of a parametric acoustic array was conceived". In: Acoustical Physics 45 (1999), pp. 611-618 (cit. on p. 12). 



\section{Summary}

\{What?\} Disorder of size (polydispersity) and mass of discrete elements or particles in randomly structured media (e.g., granular matter such as soil) has numerous effects on materials' sound propagation characteristics. The influence of disorder on energy and momentum transport during vibration propagation (mechanical/sound wave), the sound wave speed and its low-pass frequency-filtering characteristics is the subject of this study. \{Why?\} The goal is understanding the connection between the particle-microscale disorder and dynamics and the system-macroscale wave propagation, which can be applied to nondestructive testing, seismic exploration of buried objects (oil, mineral, etc.) or to study the internal structure of the Earth. \{How?\} The mechanical wave/vibration propagating through granular media exhibits a specific signature in time; a coherent pulse or wavefront arrives first with multiply scattered waves (coda) arriving later. The coherent pulse is of low frequency nature and micro-structure independent i.e. it depends only on the bulk properties of the disordered granular sample, the sound wave velocity and hence, bulk and shear moduli. The coda or the multiply scattered waves are of high frequency nature and are micro-structure dependent. Numerical and stochastic techniques for 1-D, 2-D discrete element systems and experiments with 1-D photoelastic particles constituting a granular chain have been employed to isolate and study different modes of the propagating waves (namely, $P$ and $S$ waves), disorder dependent dispersion relations, sound wave velocity and diffusive transport of spectral energy. \{Results\} Increase in mass disorder (where disorder has been defined such that it is independent of the shape of the probability distribution of masses) decreases the sound wave speed along a granular chain. Averaging over energies associated with the eigenmodes can be used to obtain better quality dispersion relations and can be formulated in a way to give a Master Equation of energy in terms of wavenumber or frequency which identifies the switching and cross-talk of energy between different frequency bands; these dispersion relations confirm the decrease 
in pass frequency and wave speed with increasing disorder acting opposite to the wave acceleration close to the source. Also, it is observed that an ordered granular chain exhibits ballistic propagation of energy whereas, a disordered granular chain exhibits more diffusive like propagation, which eventually becomes localized at long time periods. 


\section{Samenvatting}

\{Wat?\} Wanorde van grotte (polydispersiteit) en massa van deeltjes of discrete elementen in ongestructureerde media (bijv. Korrelige materialen zoals grond) heeft vele effecten op de geluid beweging. De invloed van wanorde op energie en impulse transport tijdens trillingsvoortplanting (mechanische/geluid), de geluidssnelheid en de doorgang karakteristieken in het lage frequentie domijn is het onderwerp van deze dissertatie. \{Waarom?\} Het doel is om inzicht te krijgen in de relatie tussen de micro-schaal wanorde van deeltjes en de dynamica en de systeemmacro geluid beweging, die kan worden toegepast op niet-destructieve experimenten, seismische verkenning van begraven objecten (olie, mineralen, enz.) of om de interne structuur van de aarde te bestuderen. \{Hoe?\} De mechanische golf/vibratie die zich voortplant door granulaire media vertoont een specifieke gedrag in de tijd; een coherente puls of een golffront arriveert als eerste met meerdere verstrooide golven (coda) later. De coherente puls heeft een laagfrequent karakter en is onafhankelijk van de microstructuur, d.w.z deze hangt alleen af van de bulkeigenschappen van het monster van ongeordende granulaire materiaal, de geluidssnelheid en daarom bulk- en schuifmoduli. De coda hebben een hoogfrequent karakter en zijn afhankelijk van de microstructuur. Numerieke en stochastische technieken voor 1-D, 2-D discrete elementensystemen en experimenten met 1-D foto-elastische deeltjes die een granulaire keten vormen, zijn gebruikt. Het helpt voor het isoleren en bestuderen van verschillende wijzen van de voortplantingsgolven (namelijk P en S golven), dispersierelaties die afhankelijk zijn van wanorde, geluidssnelheid en diffusief transport van vermogenspectrum. \{Resultaten\} Toename van massa wanorde (waarbij wanorde zodanig is gedefinieerd dat deze onafhankelijk is van de vorm van de kansverdeling van massa's) verlaagt de geluidssneheid door een granulaire keten. Middelen van energie met de eigenmodi zijn geassocieerd, kunnen worden gebruikt om een hogere kwaliteit dispersierelaties te verkrijgen en kunnen worden 
geformuleerd om een Master-vergelijking van energie te geven in termen van golfgetal of frequentie die het uitwisselen en overspraak van energie tussen verschillende frequentiebanden identificeert; deze dispersierelaties bevestigen de afname van bandbreedte en geluidssnelheid met toenemende wanorde die tegengesteld is aan de versnelling nabij de bron. Ook wordt waargenomen dat een geordende granulaire keten ballistische voortplanting van energie vertoont, terwijl een ongeordende granulaire keten meer diffusie-achtige voortplanting vertoont, die uiteindelijk na lange tijd wordt gelokaliseerd. 


\section{Acknowledgments}

First and foremost, I would like to thank my supervisor, mentor and guide during my PhD tenure, Prof. Stefan Luding. It was his utter faith in me and his constant guidance that I was able to write and finish this thesis. I gained a lot on both personal as well as professional front while working with him. Next, I would like to acknowledge the warm hospitality and caring nature of Gehrlinde, the moments spent and conversations made always seem insufficient. I would like to thank Dr. Orion Mouraille, Dr. Brian Lawney and Sebastian Sterl for their previous research work which served as a valuable indicator for me to further my research work. I would like to thank Kuni-san for the scientific/nonscientific discussions and being an excellent mentor and collaborator. I would also take this opportunity to thank Megumi-san, Miku and Haru for their hospitality in Sendai, Japan. I hope I meet them again soon. I would like to thank Amalia Thomas and Dr. Nathalie Vriend from Cambridge University for sharing their experimental data related to wave propagation in photoelastic disks. I would also like to thank Dr. Vanessa Magnanimo for her challenging and goal oriented research questions which assisted me in setting the milestones of my $\mathrm{PhD}$ research work. I thank Dr. Anthony Thornton for his motivational discussions. I thank Dr. Thomas Weinhart and Dr. Wouter den Otter for finding time to answer my scientific questions and clearing my doubts, working with them during Programming in Engineering courses was a good learning experience. I thank my Dutch colleagues Bert, Marnix and Irana for the discussions, suggestions and assistance during many situations. Special thanks to Bert for helping me out with the Dutch translation of the Summary of this thesis. Thanks would not be enough for the co-operation and inexhaustive support provided by Sylvia during the past 4 years.

My acknowledgements would be incomplete without the mention of NWO/former FOM organization for providing the infrastructure in the form of courses, 
annual reports, etc., which assisted me during my $\mathrm{PhD}$. The Nijenrode Business Course (offered by FOM) was the best course during my PhD course work, it gave me the opportunity to meet such wonderful people amongst whom some have become lifelong friends: Damien, Stein, Jaoge, Ning, Andrew, Sebastian and of course Martijn. I hope I get more time and opportunity in my life to enjoy their company.

Now comes the opportunity and the paragraph for which I had been waiting for a very long time. I would really like to thank Kia, (my paranymph) my blood brother for his selfless friendship, I would also like to thank him for introducing me to such wonderful persons as Behzad, Aleks and Somik, it was their friendship that made possible for me to call Europe, home (special thanks to Behzad for the Istanbul trip, Somik for the Paris/Prague trip and Aleks for a short duration stop over at Linz). My other blood brothers Anil and Mrinal, who reside in India always provided me constant support during my $\mathrm{PhD}$, it is always fascinating and motivational to enter into scientific/non-scientific discussions with Anil. I thank Ahmed (my other paranymph) for accompanying me during my trip to Japan and being a fellow anime enthusiast. Sorry that I spoiled many series for you! I would also like to thank my housemates Alex, Alessandra, Justin and Dikzak for celebrating and giving me company during numerous occasions. I would also like to thank Dr. Sudeshna Roy (who was like an elder sister), Dr. Hoongyang Cheng, Dr. Valantis Tsinginos, Hao Shi, Durai, Giorgio, Thejas, Ali and Sara for helping me during numerous occasions. A special thanks goes to the old PhD students Dr. Shushil, Dr. Abhineendra, Dr. Nishant and Dr. Vitaliy who provided a warm and caring environment during the early stages of my $\mathrm{PhD}$.

Mere thanks would never suffice the efforts and sacrifices made by my parents Renuka (mother) and Subhas (father) for me. They acted as an anvil for me, I hope I can stand up to their expectations and make them proud. Finally, I would like to thank Shell global and University of Twente for providing the funding and necessary infrastructure without which my $\mathrm{PhD}$ would not have been possible. 


\title{
Curriculum Vitae
}

\author{
6 March 1989 : Date of Birth \\ May - July 2011 : Internship \\ Istituto Nazionale di Geofisica e Vulcanologia \\ (I.N.G.V) \\ Naples, Italy. \\ July 2012 - January 2013 : Master's Dissertation \\ University of Udine, Italy \\ September 2013 : Integrated Master of Technology \\ Geophysical Technology, \\ (Bachelor + Master) \\ I.I.T Roorkee, India
}




\section{List of Publications}

\section{Journals}

- R.K. SHRIVASTAVA and S. LUDING. "Effect of disorder on bulk sound wave speed: a multiscale spectral analysis". In: Nonlinear Processes in Geophysics 24.(3) (2017), pp. 435-454 [177].

- R.K. SHRIVASTAVA and S. LUDING. "Energy Propagation with Distance and Across Wavenumbers in a Granular Chain". In Preparation (2018).

- K. SAITOH, R.K. SHRIVASTAVA and, S. LUDING. "Rotational sound in disordered granular materials". In Preparation (2018) [163].

\section{Conference Proceedings}

- R.K. SHRIVASTAVA and S. LUDING. "Wave propagation of spectral energy content in a granular chain". In: EPJ Web of Conferences Vol. 140. EDP Sciences. 2017, p. 02023 [178].

- R.K. SHRIVASTAVA, A. THOMAS, N. VRIEND, and S. LUDING. "Long Wavelength Coherent Pulse of Sound Propagating in Granular Media". In: World Academy of Science, Engineering and Technology, International Journal of Mechanical, Aerospace, Industrial, Mechatronic and Manufacturing Engineering 11.(10) (2017), pp. 1694-1701 [179].

\section{Master Thesis}

- R.K. SHRIVASTAVA. "Application of Support Vector Machines for Seismogram Analyses and Differentiation". Master Thesis. I.I.T Roorkee, 2013. 